\title{
DESARROLLO E IMPLEMENTACIÓN DE ALGORITMOS PARA LA OPTIMIZACIÓN ENERGÉTICA EN TIEMPO REAL DE REDES HIDRÁULICAS A PRESIÓN
}

Tesis Doctoral

\author{
Presentada por: \\ Joan Carles Alonso Campos
}

Dirigida por:

Dr. Fernando Martínez Alzamora

Dr. Miguel Ángel Jiménez Bello

Universitat Politècnica de València

Valencia, noviembre 2021 



\section{AGRADECIMIENTOS}

Quisiera agradecer, en primer lugar, a la Generalitat Valenciana por su programa de financiación para investigadores en formación, VAL I+D. Sin duda, el respaldo financiero fue fundamental a la hora de tomar la decisión de emprender el camino de la investigación.

Quisiera agradecer a mis directores su esfuerzo y dedicación a la hora de guiarme en esta aventura. Gracias Fernando por tus ideas y, sobre todo, por compartir de modo tan generoso tus conocimientos. Gracias Miguel Ángel por tus consejos, tu inestimable ayuda para sacar adelante los resultados y por animarme en los momentos bajos.

Gracias a mis compañeros en Global Omnium, especialmente a Pilar y a Marta, por ayudarme en el acercamiento a las problemáticas del mundo real.

También me gustaría agradecer a mis compañeros del REDHISP, Vicente, Óscar, Igor, Juan Manuel, los buenos momentos, así como los bienvenidos consejos y sugerencias.

Gracias a mi familia, por su apoyo incondicional y por ayudarme a creer en mí. 



\section{RESUMEN}

El objetivo general de la presente Tesis es investigar metodologías que permitan obtener en tiempo real los parámetros de operación de redes hidráulicas a presión que minimicen el consumo y/o el coste energético, garantizando el cumplimiento de las condiciones de funcionamiento necesarias para una adecuada calidad del servicio.

Al tratarse del ámbito de la operación diaria de la red, una de las condiciones indispensables que deben reunir los métodos de optimización es una respuesta lo suficientemente rápida como para que no solo se pueda disponer de las soluciones más convenientes en el momento de ejecutar las consignas de operación, sino que además se habilite un procedimiento flexible que permita dar respuesta a posibles cambios en las predicciones o eventos que puedan producirse.

Se ha abordado de manera aislada la optimización energética de los subsistemas de transporte de agua y la de los subsistemas de distribución debido a las distintas características que se pueden observar en ellos.

En la parte relativa a los subsistemas de distribución, particularizada al caso de un sistema de riego con bombeo directo a red, se han explorado los métodos metaheurísticos de optimización, realizando varias aportaciones originales orientadas a la mejora en la eficiencia computacional de los mismos, debido a la necesidad de obtener una respuesta rápida compatible con la toma de decisiones en tiempo real.

En cuanto a los subsistemas de transporte, se ha explorado la aplicabilidad del método determinista de optimización por programación lineal, a la vista de las importantes ventajas que presenta respecto al resto de métodos generales de optimización.

Asimismo, en el contexto de los subsistemas de transporte, se ha trabajado en la definición de una heurística basada en el cálculo del coste energético y/o económico del agua entregada en los puntos de consumo y almacenada en los depósitos intermedios, que ha permitido formular un algoritmo voraz para la optimización energética en cada instante de tiempo. Este método ha conseguido igualar el desempeño alcanzado mediante la programación lineal y se espera que ofrezca unas mejores capacidades en sistemas con un comportamiento marcadamente no lineal, así como también una mejor adaptación a problemas de optimización con la participación de energías renovables. 



\section{RESUM}

L'objectiu general de la present Tesi és la investigació de metodologies que permeten obtindre en temps real els paràmetres d'operació de xarxes hidràuliques a pressió que minimitzen el consum i/o el cost energètic, garantint el compliment de les condicions de funcionament necessàries per a una adequada qualitat del servei.

En tractar-se de l'àmbit de l'operació diària de la xarxa, una de les condicions indispensables que han de reunir els mètodes d'optimització és una resposta prou ràpida com perquè no sols es puga disposar de les solucions més convenients en el moment d'executar les consignes d'operació, sinó que a més s'habilite un procediment flexible que permeta donar resposta a possibles canvis en les prediccions o esdeveniments que puguen produir-se.

S'ha abordat de manera aïllada l'optimització energètica dels subsistemes de transport d'aigua i la dels subsistemes de distribució (reg per injecció directa) a causa de les diferents característiques que es poden observar en ells.

En el treball amb els subsistemes de distribució s'han explorat les possibilitats que ofereixen els mètodes meta-heurístics d'optimització, realitzant diverses aportacions originals orientades a la millora en l'eficiència computacional dels mateixos a causa de la necessitat d'obtindre una resposta més ràpida que siga compatible amb la presa de decisions en temps real.

Quant als subsistemes de transport, s'ha explorat l'aplicabilitat del mètode determinista d'optimització per programació lineal a la vista dels importants avantatges que presenta respecte a la resta de mètodes generals d'optimització.

Així mateix, en el context dels subsistemes de transport, s'ha treballat en la definició d'una bona heurística basada en el càlcul del cost energètic i/o econòmic de l'aigua entregada en els punts de consum i en els dipòsits intermedis, que ha permés formular un mètode voraç per a l'optimització energètica en cada instant de temps. Aquest mètode ha aconseguit igualar l'acompliment aconseguit mitjançant la programació lineal i s'espera que oferisca unes millors capacitats en sistemes amb un comportament més marcadament no lineal, així com també una millor adaptació a problemes d'optimització amb participació d'energies renovables. 



\section{SUMMARY}

The general objective of this Thesis is the research of methodologies to obtain in real time the operating parameters of pressurized hydraulic networks that minimize energy consumption and/or cost, ensuring compliance with the operating conditions necessary for an appropriate quality of service.

Since this is the field of daily network operation, one of the indispensable conditions that optimization methods must meet is a response fast enough so that not only the most convenient solutions are available at the time of executing the operating instructions, but also a flexible procedure is provided to allow a response to possible changes in the predictions or events that may occur.

The energy optimization of the water transport subsystems and that of the distribution subsystems (direct injection irrigation) have been approached separately due to the different characteristics that can be observed in them.

In the work with distribution subsystems, the possibilities offered by metaheuristic optimization methods have been explored, making several original contributions aimed at improving their computational efficiency due to the need to obtain a faster response that is compatible with real-time decision making.

Regarding transport subsystems, the applicability of the deterministic method of optimization by linear programming has been explored in view of the important advantages it presents with respect to the rest of the general optimization methods.

Also, in the context of transport subsystems, there has been a work on the definition of a good heuristic based on the calculation of the energy and/or economic cost of the water delivered at the consumption points and intermediate reservoirs, which has allowed to formulate a greedy method for energy optimization at each time instant. This method has been able to match the performance achieved by linear programming and is expected to offer better capabilities in systems with a more marked non-linear behaviour, as well as a better adaptation to optimization problems involving renewable energies. 



\section{ÍNDICE}

AGRADECIMIENTOS i

RESUMEN —__ iii

RESUM —_

SUMMARY _ _ _ vii

ÍNDICE DE FIGURAS _____ xiii

ÍNDICE DE TABLAS ___ _ _

CAPÍTULO 1. INTRODUCCIÓN_______________ 1

1.1 Motivación y objetivos ___ 1

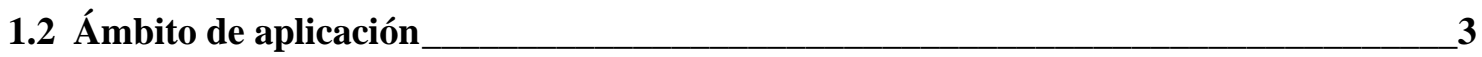

1.3 Problemática y retos de la operación diaria. Estado del arte ___ 5

1.3.1 Cálculos energéticos e indicadores en sistemas de distribución de agua ___ 6

1.3.2 Métodos para la reducción del consumo energético en SDA ___ 8

1.3.3 Reducción del consumo energético en el caso particular de redes de riego ___ 10

1.4 Estructura de la Tesis ___ 12

CAPÍTULO 2. HERRAMIENTAS UTILIZADAS ___ 15

2.1 Generalidades de los métodos de optimización____ 15

2.1.1 Métodos deterministas ___ 15

2.1.2 Métodos heurísticos _ـ_ 17

2.1.3 Métodos metaheurísticos____ 17

2.2 Software de simulación hidráulica: EPANET ___ 21

2.2.1 Modelado matemático______ 21

2.2.2 Toolkit de EPANET ___ _ _ 24

2.2.3 Modelización hidráulica para la optimización energética____ 25

CAPÍTULO 3. OPTIMIZACIÓN ENERGÉTICA DE REDES DE RIEGO CON BOMBEO DIRECTO — 27

3.1 Introducción a las particularidades de las redes de riego 27

3.2 Metodología general de optimización energética en redes de riego _ـ 30

3.2.1 Definición de una red de riego, con miras a su optimización ____ 30

3.2.2 Planteamiento del problema base de optimización ___ 32

3.2.3 Desdoblamiento del problema en periodo de bombeo y periodo de gravedad ___ 38 
3.2.4 Caso de estudio. Comunidad de regantes de "El Realón” ____ 40

3.2.5 Conclusiones derivadas de aplicar la metodología descrita ___ 50

3.3 Mejoras en el método de optimización para acelerar los cálculos ___ 51

3.3.1 Descripción del algoritmo ___ 52

3.3.2 Caso de estudio. Comunidad de regantes de Peñarroya____ 58

3.3.3 Resultados ___ 61

3.3.4 Conclusiones ___ 65

3.4 Mejoras en el método de maximización del volumen entregado por gravedad __ 66

3.4.1 Descripción del método ___ 66

3.4.2 Resultados de la aplicación de la variante al caso de estudio de "El Realón" ___ 68

3.4.3 Conclusiones al método mejorado para maximizar el volumen por gravedad___ 71

3.5 Conclusiones generales_____ 71

CAPÍTULO 4. OPTIMIZACIÓN ENERGÉTICA DE SISTEMAS DE TRANSPORTE DE AGUA__ 73

4.1 Introducción __ 73

4.2 Descripción del método por programación lineal.___ 74

4.2.1 Definición de los subsistemas ___ 75

4.2.2 Consideraciones sobre la optimización lineal ___ 78

4.2.3 Planteamiento del problema de optimización lineal____ 79

4.2.4 Cálculo de las funciones de transferencia ___ 84

4.2.5 Obtención del precio de la energía____ 85

4.2.6 Comparación de resultados __ 87

4.3 Criterio para el establecimiento del valor final del nivel de los depósitos____ 88

4.4 Caso de estudio ___

4.5 Resultados ___

4.6 Conclusiones ___ 98

CAPÍTUlo 5. MÉTodos heurísticos ___ 101

5.1 Introducción ___ 101

5.2 Cálculo de los flujos energéticos en redes de distribución de agua ___ 102

5.2.1 Descripción del método ___ _ _ 102

5.2.2 Ejemplo de aplicación ___ 119

5.2.3 Conclusiones ___ 122

5.3 Metodología de optimización basada en el coste del agua almacenada___ 123 
5.3.1 Equiparación de la densidad de energía a la densidad de coste 124

5.3.2 Función de mezcla en los depósitos 126

5.3.3 Algoritmo voraz de optimización 128

5.4 Resultados 130

5.5 Conclusiones 132

CAPÍTULO 6. TRATAMIENTO DE LOS DATOS DE CAMPO 135

6.1 Introducción 135

6.2 Detección y clasificación de los datos anómalos 136

6.3 Metodología de restitución de los datos anómalos 137

6.3.1 Restitución de datos erróneos a partir de patrones 138

6.3.2 Combinación de distintas granularidades en las series de datos 139

6.3.3 Caso particular. Ajuste de las medidas de los caudalímetros en base al balance caudales 140

6.3.4 Caso de que algún caudal sea calculado 142

CAPÍTULO 7. CONCLUSIONES 145

7.1 Conclusiones generales 145

7.2 Aportaciones originales 147

7.3 Publicaciones 147

7.3.1 Revistas JCR 147

7.3.2 Congresos 148

7.3.3 Libros 148

7.4 Discusión y limitación de resultados 149

7.5 Trabajos futuros 150

7.5.1 Cambio de paradigma en la optimización energética de redes de riego 150

7.5.2 Formulación del balance global de energías a lo largo de un periodo de tiempo _ 151

7.5.3 Definición de nuevos indicadores energéticos 151

7.5.4 Mejora del sistema de filtrado de datos 152

BIBLIOGRAFÍA 153 



\section{ÍNDICE DE FIGURAS}

Figura 1. Esquema ilustrativo de la identificación de variables para el subproblema de maximizar el volumen entregado por gravedad.

Figura 2. Vista general de la red de riego de Realón.

Figura 3. Curvas de presión y rendimiento en función del caudal de las bombas BEV-1226/4

Figura 4. Curvas de presión y rendimiento en función del caudal para las bombas con 3 rodetes.

Figura 5. Curvas de altura-caudal de las bombas para la regulación de la estación de bombeo del cabezal de Realón.

Figura 6. Caudal total entregado por gravedad a la red en función del tiempo. Metodología 1. 48

Figura 7. Demanda de potencia en función del tiempo. 49

Figura 8. Caudal entregado en función del tiempo 50

Figura 9. Representación del conjunto de soluciones no dominadas dentro del conjunto de la población al finalizar distintas generaciones. Las soluciones seleccionadas en cada frente se han remarcado con un círculo.

Figura 10. Valor de los dos objetivos para el individuo seleccionado del Frente de Pareto (puntos redondeados de la Figura 9) al final de la evaluación de cada generación.

Figura 11. Esquema de la red de riego del sector III de Peñarroya. 58

Figura 12. Curva de rendimiento global de la estación de bombeo del sector III de la comunidad de regantes del Pantano Estrecho de Peñarroya (C. Real), en función del caudal total bombeado, calculada para obtener el mejor rendimiento a partir de las curvas reales de cada bomba, con una presión de consigna de 38 m.c.a.. 59

Figura 13. Representación de los datos del valor objetivo mediante el gráfico de caja y bigotes.

Figura 14. Valor del ratio entre el tiempo de computación de la parte inevitablemente secuencial del programa y el tiempo total de cómputo.

Figura 15. Diagrama de flujo del algoritmo para la maximización del volumen entregado por gravedad.

Figura 16. Resultados de simular la programación de riego actual. 68

Figura 17. Resultados optimización mediante la metodología descrita en apartado 3.2.3.. 69 
Figura 18. Resultados optimización mediante la nueva metodología

Figura 19. Esquema de los elementos que componen un subsistema de optimización dentro de una red de distribución de agua.

Figura 20. Estructura del archivo de configuración propuesto para la optimización mediante programación lineal. 78

Figura 21. Ejemplo de archivo descargado de OMIE que contiene los precios de la energía hora a hora para el día 14/01/2021 86

Figura 22. Esquema hidráulico del ejemplo utilizado para la validación del criterio de establecimiento del valor final del depósito.

Figura 23. Precio de la energía del mercado español del día 24/03/2015. Fuente: www.omie.es 89

Figura 24. Niveles del depósito para cada iteración de la optimización. 90

Figura 25. Precio hipotético de la electricidad para dos días consecutivos 92

Figura 26. Trayectorias del depósito en el ejemplo propuesto 92

Figura 27. Estación de bombeo del subsistema a optimizar. 93

Figura 28. Esquema del subsistema a optimizar. 94

Figura 29. Caudal trasegado por las bombas B1 y B2, y por la válvula VReg en el escenario inicial que reproduce la operación real del día 1-6-21

Figura 30. Evolución del nivel en los depósitos dep2 y dep3 en el escenario inicial que reproduce la operación real del día 1-6-21 95

Figura 31. Precio de la energía para el día 1-6-21 ajustado a la tarifa negociada por la compañía gestora del sistema de distribución de agua. 96

Figura 32. Configuración del subsistema a optimizar. 96

Figura 33. Resultado obtenido mediante programación lineal para la optimización del subsistema.

Figura 34. Caudal trasegado por las bombas B1 y B2, y por la válvula VReg en el escenario optimizado para el día 1-6-21

Figura 35. Evolución del nivel en los depósitos dep2 y dep3 en el escenario optimizado para el día 1-6-21 98

Figura 36. Modelo de mezcla en el punto c de las fracciones de caudal procedentes de la fuente $\mathrm{s}$ 
Figura 37. Modelo de mezcla en el punto de consumo c para la energía procedente de la bomba p, transportada a lo largo de un tubo de corriente con una densidad

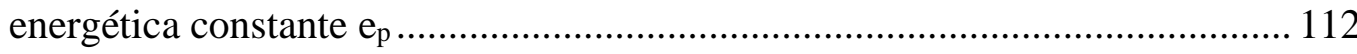

Figura 38. Modelo de mezcla en el nudo de consumo c para la energía procedente de la bomba $\mathrm{p}$ transportada por el caudal total, con distinta densidad energética para cada nudo.

Figura 39. Red de ejemplo que incluye bombas de alimentación y sobrepresoras, embalses, nudos de demanda y un depósito con caudal entrante y saliente simultáneamente.

Figura 40. Alturas piezométricas y caudales para el instante de estudio. 120

Figura 41. Caudal trasegado por las bombas B1 y B2, y por la válvula VReg en el escenario optimizado mediante el algoritmo voraz..... 131

Figura 42. Evolución del nivel en los depósitos dep2 y dep3 en el escenario optimizado mediante el método voraz 131 



\section{ÍNDICE DE TABLAS}

Tabla 1. Cálculo de pérdidas en tuberías (pérdidas en pies y caudal en cfs).

Tabla 2. Ejemplo de codificación de un cromosoma o vector de variables del problema de optimización.

Tabla 3. Resumen valores principales antes y después de la optimización

Tabla 4. Coeficiente para el cálculo de la penalización por exceso de potencia en tarifas de seis periodos 54

Tabla 5. Distribución anual de los periodos horarios para la tarifa 6.1A 60

Tabla 6. Resumen de estadísticos para el conjunto de escenarios planteados 61

Tabla 7. Resumen de la mejora alcanzada por una de las soluciones respecto a la

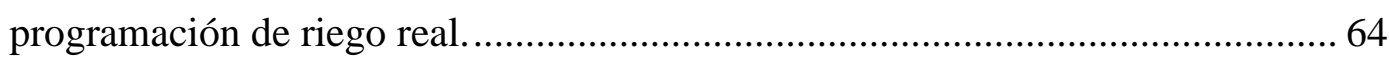

Tabla 8. Niveles del depósito 91

Tabla 9. Resumen comparativo entre los resultados de la simulación de la operación real y el escenario propuesto por el optimizador lineal .98

Tabla 10. Resultados en cada nudo de consumo de proporciones de caudal procedentes de cada fuente y densidades de energía relacionadas con las bombas y las pérdidas de carga.

Tabla 11. Resultados del balance de energía a nivel de nudo en un instante dado para los nudos de demanda. 122

Tabla 12. Resumen comparativo entre los resultados de la simulación de la operación real, el escenario propuesto por el optimizador lineal y el escenario propuesto por el algoritmovoraz. 132 



\section{CAPÍtulo 1.}

\section{INTRODUCCIÓN}

\subsection{Motivación y objetivos}

La motivación de la presente Tesis Doctoral se genera bajo el contexto de las directrices estratégicas existentes encaminadas a la búsqueda de la sostenibilidad a nivel global. Una de las líneas generales de acción es la implantación de medidas para el fomento del ahorro energético. Además, el constante aumento en el precio de las tarifas eléctricas evidencia la necesidad de mejorar, en la medida de lo posible, la toma de decisiones por parte de las personas encargadas de la gestión de los sistemas de distribución de agua. La presente Tesis se enfoca en la investigación de metodologías que permitan obtener los parámetros de operación de redes hidráulicas a presión que minimicen el consumo y/o el coste energético, garantizando el cumplimiento de las condiciones de funcionamiento.

El objetivo general que se pretende alcanzar es el diseño de metodologías que no solo minimicen el consumo energético, sino que además permitan llevar a cabo dicha optimización en tiempo real ${ }^{1}$, a la vez que se garantizan una serie de restricciones relacionadas con la calidad del servicio.

El desarrollo de la Tesis se apoyó, como punto de partida, en varias investigaciones realizadas en el grupo de Redes Hidráulicas y Sistemas a Presión (REDHISP), perteneciente al Instituto de Ingeniería del Agua y Medio Ambiente (IIAMA), donde se enmarca la propia

\footnotetext{
${ }^{1} \mathrm{O}$, al menos, en un tiempo suficientemente reducido como para que los resultados sean de utilidad en la toma de decisiones de la operación diaria.
} 
Tesis. Dichos trabajos aportaron metodologías que permitían reducir teóricamente el consumo energético de redes de riego, pero presentaban algunas dificultades a la hora de su implantación, y de su extensión para considerar otros tipos de redes.

Para solucionar este aspecto, se han explorado primeramente distintas rutinas de cálculo que permitan llevar a cabo la optimización en tiempo real, para poder considerar cambios en variables no predecibles o que deben actualizarse periódicamente (fallos de elementos, cambios en tarifas, cambios en la climatología, variaciones en la demanda, etc.). Este es uno de los aspectos novedosos que aporta la presente Tesis.

Además, se ha extendido el campo de aplicación de los optimizadores a las redes de transporte de agua, con una problemática algo distinta a la tratada hasta ahora, para contemplar además la presencia de almacenamientos en la gestión del agua, cada vez más necesarios en la reutilización de los recursos y la entrada de la energía fotovoltaica.

Por último, se ha mejorado el tratamiento de las diferentes tarifas eléctricas en el estudio, de modo que el modelo contemple también como variables el término de potencia y las penalizaciones por exceso de potencia, así como los precios de la energía variables hora a hora (consumos en alta), o bien los regulados por contrato para los distintos periodos tarifarios (consumo en media y baja potencia).

Para la consecución del objetivo general de la Tesis, se ha tratado de cumplir además los siguientes objetivos específicos:

- Conocer las metodologías existentes para la optimización energética de redes hidráulicas y su aplicación a casos reales.

- Desarrollar nuevas metodologías que aporten mejoras a las existentes en cuanto a resultados, rapidez de cálculo y capacidad para optimizar redes más complejas.

- Trasladar la experiencia a redes de abastecimiento, además de las redes de riego.

- Dotar de generalidad a las diferentes metodologías para que sean de aplicabilidad en casos muy diversos.

- Diseñar en base a las nuevas metodologías los algoritmos necesarios para realizar los cálculos, e implementarlos en una plataforma informática no dependiente de software comercial.

- Aplicar los resultados a casos de estudio reales para la validación de los desarrollos. 


\section{2 Ámbito de aplicación}

Un sistema de distribución de agua (SDA) es un conjunto de elementos físicos conectados que permiten transportar agua desde las fuentes o puntos de suministro hasta los puntos en que se encuentran los consumos o demandas. El ámbito de aplicación de las metodologías propuestas en la presente Tesis son los SDA que transportan agua dulce para consumo humano (potable) o para riegos, discurriendo por conductos cerrados a una presión mayor que la atmosférica.

En general, para transportar el agua en condiciones adecuadas se necesita un cierto aporte energético para vencer los desniveles topográficos, las pérdidas de carga que se producen en tuberías, válvulas y accesorios, y para finalmente dotar al agua de una presión mínima en los puntos de consumo. La energía aportada puede encontrarse de forma natural, si las fuentes de suministro se encuentran a mayor elevación que los puntos de consumo, o debe comunicarse a través de equipos de bombeo. Estos elementos son precisamente los que incurren en un coste de operación, ya que deben ser accionados por una fuente de energía externa, que normalmente será la red eléctrica.

Un buen diseño de los SDA es fundamental para minimizar los requerimientos de aportes externos de energía en todo el ciclo de vida del sistema. Sin embargo, en los sistemas ya en explotación todavía existen posibilidades de reducir el consumo y/o el coste en energía incurrido en el transporte de agua. En la gestión de los SDA, día a día se toman decisiones operativas que pueden tener mayor o menor impacto sobre el consumo de energía. En general, se puede sintetizar que las decisiones de operación de una red, ya sea directa o indirectamente, afectan básicamente a dos tipos de elementos: las bombas y las válvulas.

En los distintos capítulos de esta Tesis se presentarán una serie de metodologías mediante las que se pueden calcular u obtener los parámetros de operación de los SDA óptimos desde el punto de vista energético. Naturalmente, este objetivo principal debe ser compatible con los requisitos relacionados con la garantía del servicio.

Al tratarse del ámbito de la operación diaria de la red, una de las condiciones indispensables que deben reunir los métodos de optimización es una respuesta lo suficientemente rápida como para que no solo se pueda disponer de las soluciones más convenientes en el momento de ejecutar las consignas de operación, sino que además se habilite un procedimiento flexible que permita dar respuesta a posibles cambios en las 
predicciones o eventos que puedan producirse. Tal como se verá más adelante, este requisito supone uno de los retos más importantes a la hora de resolver los problemas de optimización energética de la operación diaria de los SDA.

En cuanto a la estructura de los SDA, debe establecerse una diferenciación entre los subsistemas de transporte y los de distribución. Los subsistemas de transporte toman el agua desde las fuentes de suministro (plantas potabilizadoras, embalses, ríos, etc.) y la elevan hasta los depósitos de regulación. Desde estos depósitos se realiza después la distribución hasta los puntos de consumo. Normalmente, los subsistemas de transporte contarán con equipos de bombeo para elevar el agua hasta las cotas en las que se encuentren los depósitos. En el caso de los subsistemas de distribución, aunque en el caso más general el agua discurra por gravedad hasta los puntos de consumo, tal como se verá más adelante, existen subsistemas que requieren el apoyo de un bombeo directo a red para suministrar el agua en las condiciones de presión requeridas. Esta tipología se observa con frecuencia en sistemas de riego por no tratarse de un suministro crítico.

Con respecto a la formulación del problema de optimización, a la hora de optimizar la operación de un sistema completo, lo ideal es desacoplar el transporte de la distribución y tratar cada caso como un problema aislado. Para que esto sea posible, debe darse la situación de que, en el sistema real de estudio, la capacidad de los depósitos intermedios sea lo suficientemente grande como para que los posibles cambios en las demandas satisfechas por estos no afecten a los resultados de la optimización del subsistema de transporte. En la presente Tesis, se ha asumido esta hipótesis y se han propuesto metodologías adaptadas a sistemas de transporte y a sistemas de distribución por separado. La asunción se fundamenta en dos hechos: por un lado, en los sistemas de agua potable, las demandas urbanas suelen ser muy estables, tanto los volúmenes totales diarios como su modulación horaria, así que es esperable que las pequeñas variaciones que puedan darse no tengan suficiente entidad como para afectar al sistema de transporte; por otro lado, los sistemas de riego normalmente cuentan con balsas con una capacidad que cubre las necesidades de varias jornadas de riego, de modo que las leyes de consumo tampoco afectarían a las reglas del transporte, siempre que se optimice con un horizonte temporal igual o inferior a 24 horas.

El enfoque del problema de optimización en ambos casos es distinto. En los sistemas de transporte los depósitos juegan un papel fundamental, permitiendo que se pueda programar el arranque o paro de las bombas en los instantes más idóneos, siempre que el 
nivel en los mismos se mantenga dentro de unos límites prefijados. Los depósitos se dimensionan con una capacidad suficiente para satisfacer desde la mitad de la demanda diaria hasta la demanda de uno o más días, según el número de habitantes suministrados (CEDEX 2017), lo que hace posible desarrollar estrategias de optimización energética.

Por el contrario, un sistema de distribución apoyado por un bombeo de inyección directa deberá satisfacer en cada momento el caudal demandado. En este caso, si se trata de un sistema urbano, la libertad de actuación en el contexto energético es muy limitada, restringiéndose únicamente a las posibles mejoras en la programación interna del controlador de la estación de bombeo, cosa que no forma parte de los objetivos de la presente Tesis. Pero, si se trata de un sistema de riego, las posibilidades de mejora del consumo energético son mayores, siempre que se permita una cierta capacidad de actuación a la hora de asignar las demandas, esto es, en la programación de los riegos.

Así pues, las metodologías propuestas en la presente Tesis se han orientado, por un lado, a la resolución del problema de optimización de subsistemas de transporte, desacoplados de los posibles subsistemas de distribución que pudiesen existir; y por otro, a la resolución del problema de optimización de subsistemas de distribución por inyección directa en redes de riego.

\subsection{Problemática y retos de la operación diaria. Estado del arte}

Hoy en día existe una preocupación creciente por la eficiencia energética, en particular en lo que respecta a los sistemas de distribución de agua. Muchos investigadores han propuesto distintas metodologías destinadas a la reducción del consumo de energía (Jowitt y Germanopoulos, 1992; Ormsbee y Lansey, 1994; Savic y Walters, 1997; Giacomello et al., 2013; Reca, García-Manzano, y Martínez 2015; Bou Soler, 2016; Pardo et al., 2020). Otros se han centrado más en evaluar la eficiencia energética del sistema a través de un conjunto de indicadores, dejando la mejora de dicha eficiencia para un paso posterior. En el presente apartado se comenzará por la revisión de dichos indicadores (Abadía 2008; Cabrera 2010), para después tratar las contribuciones relativas a la mejora de la eficiencia. 


\subsubsection{Cálculos energéticos e indicadores en sistemas de distribución de agua}

Varios grupos de investigación han trabajado en la definición de indicadores o métricas energéticas, que son necesarias para identificar las debilidades de los SDA y, por tanto, definir las mejores estrategias para mejorar su eficiencia. El primer enfoque usado para evaluar el consumo de energía en un sistema de agua era considerar la energía consumida por las bombas y el volumen de agua entregado, calculado para todo el sistema completo, definiendo así la intensidad de energía como la cantidad total de energía requerida para el uso de una cantidad dada de agua (Wilkinson, 2000). Este indicador es útil para comparar diferentes escenarios o acciones de mejora en un SDA determinado, aunque no es significativo en sí mismo, ya que no ofrece una idea de cuán eficiente es el sistema, es decir, cómo de lejos está del óptimo.

Pelli y Hitz (2000) definieron el término energía mínima como la "menor energía teóricamente necesaria para transportar el agua desde los puntos de producción hasta los puntos de consumo a una presión operativa de aproximadamente 6 bar". Este término se calculó como la diferencia entre la energía potencial del agua suministrada y la energía potencial del agua entregada, más la energía necesaria para elevar la presión hasta 6 bar. A continuación, se definieron dos indicadores: el índice de estructura y el índice de calidad. Este último es una medida de la eficiencia global del SDA (inverso), pero no da información sobre dónde están las principales ineficiencias.

Abadia et al. (2008) propusieron un análisis más profundo en el que la eficiencia energética global de una red se compone de dos factores: la eficiencia del bombeo (incluyendo tanto la bomba como el motor) y la eficiencia del suministro. La primera es la relación entre la potencia hidráulica suministrada y la energía eléctrica consumida, y la segunda es la relación entre la presión mínima requerida en un punto de suministro y la presión entregada por las bombas.

En un enfoque más exhaustivo, Cabrera et al. (2010) definieron el balance energético completo del sistema, teniendo en cuenta los diferentes términos energéticos, que incluyen: la energía suministrada por bombas y depósitos, la consumida por las demandas de los usuarios, la energía perdida por fugas, la disipada por fricción y la compensada por los depósitos. Propusieron dos indicadores para identificar los requerimientos de energía del sistema: la energía mínima útil, calculada a partir de la altura piezométrica requerida y la energía mínima, considerando solo la presión mínima requerida. Finalmente, definieron dos 
indicadores de contexto y cinco indicadores de eficiencia. Sin embargo, la utilidad de estas métricas se vio limitada por el hecho de que el cálculo de la energía potencial o natural, así como la entregada a los usuarios y perdida por fugas, se basa en alturas piezométricas, utilizando la cota del nudo más bajo de la red como elevación de referencia (datum de la red). Como los propios autores enfatizan, este criterio afecta a los valores de los indicadores. Pardo et al. (2013) presentaron un ejemplo de aplicación de esta metodología para realizar la auditoría energética a una red de riego. Los autores añadieron a la auditoría general un término para la disipación de energía en válvulas hidráulicas y otro para la disipación de energía en los hidrantes de riego, pero todavía no se podía relacionar el origen y el destino de la energía disgregando las distintas fuentes de energía individualmente.

En los estudios reseñados anteriormente, todas las métricas de eficiencia se basan en un objetivo ideal e inalcanzable de consumo de energía. Gay y Sinha (2012) consideraron cómo definir la energía mínima realista necesaria, pero propusieron una metodología cuya validez se limitaba a SDA simples. La misma limitación se observó en el parámetro de "energía mínima inevitable requerida", definido por Bolognesi et al. (2014), que es válido para un SDA con una sola bomba. La contribución de Cabrera et al. (2014) fue el desarrollo de esta idea mediante una metodología que estimaba un objetivo ambicioso pero alcanzable de eficiencia energética para cualquier SDA. Estos mismos autores propusieron anteriormente un procedimiento sistemático para el cálculo de la energía mínima, también basado en una auditoría energética (Cabrera et al., 2010)

El propósito de los índices definidos por Mamade et al. (2014) fue proporcionar información sobre el exceso de energía en un SDA. El cálculo de las diferentes energías, incluyendo la energía consumida por las bombas, se basó también en alturas piezométricas, por lo que el valor de estos indicadores depende de la cota elegida como referencia. Las tres métricas adicionales introducidas por Mamade et al. (2015) explican la energía que se pierde en el transporte, la proporción de energía topográfica y la relación entre el coste en energía y todos los costes relacionados con la operación, mientras que Dziedic y Karney (2015) utilizaron un enfoque similar al de Cabrera et al. (2010).

Finalmente, Hashemi, Filion y Speight (2015) describieron un balance de energía a nivel de tubería. Conceptualmente, el equilibrio es análogo al aplicado a todo el sistema, pero cada tubería tiene sus propios indicadores. 
En el CAPÍTULO 5 de la presente Tesis se propone una metodología para el cálculo del balance energético en sistemas de distribución de agua que difiere de los enfoques anteriores y tiene por objeto vincular individualmente la energía suministrada por cada fuente a su destino final, es decir, los nudos de consumo o el llenado de depósitos. Por lo tanto, en dicho capítulo se esbozará una visión innovadora de la evaluación energética de los SDA, que no ha sido tenido en cuenta con anterioridad. Mediante este enfoque, se considera que la energía suministrada por las bombas y disipada por fricción en las líneas es transportada a lo largo de la red, y finalmente la energía neta es entregada en los nudos de demanda o en las entradas a los depósitos. La diferencia entre el transporte de energía y el transporte de agua en un SDA residiría en que se asume que la energía se transmite instantáneamente en un estado estacionario, mientras que el fluido es transportado por el flujo, tardando algún tiempo en viajar a través de la red.

Mediante este enfoque se pretende obtener una información significativa que sirva de ayuda en la toma de decisiones en el ámbito de la operación y gestión diaria de los SDA, mientras que el enfoque clásico de balance energético global está orientado a auditar la red y proponer mejoras estratégicas.

\subsubsection{Métodos para la reducción del consumo energético en SDA}

Tal como se ha resumido en el apartado anterior, el consumo de energía de los sistemas de distribución de agua ha sido ampliamente estudiado durante las últimas cuatro décadas. Los esfuerzos hacia la caracterización energética de los SDA han producido metodologías bien definidas para evaluar su eficiencia energética (Cabrera et al., 2010; Dziedic y Karney, 2015; Pelli y Hitz, 2000). Por otro lado, con el fin de mejorar la eficiencia energética de los SDA, numerosos investigadores han trabajado en el desarrollo de procedimientos orientados a la optimización del diseño de los elementos de la red (Bolognesi et al., 2014; Eusuff y Lansey, 2003; Moosavian y Lence, 2011) o los parámetros de operación (Barán et al., 2005; Savic y Walters, 1997; Shi y You, 2016).

La eficacia de estos métodos de optimización se puede evaluar considerando un equilibrio entre el esfuerzo computacional, la precisión del modelo hidráulico y la optimalidad de la solución. Los investigadores han desarrollado distintas técnicas para resolver el problema de optimización. Sin embargo, no se ha demostrado que ninguna de estas metodologías sea mejor que las demás en los tres criterios mencionados. Generalmente, 
aquellas metodologías basadas en la programación matemática (programación lineal, no lineal y dinámica) requieren menos esfuerzo computacional, pero en contrapartida, o bien no pueden manejar el modelo hidráulico completo, o bien no garantizan que se alcanza el óptimo global. Por el contrario, los métodos metaheurísticos, como los algoritmos genéticos (GA) o la optimización por colonias de hormigas (ACO), son capaces de proporcionar una alta precisión en los resultados, gracias a su capacidad para combinar el propio algoritmo de optimización con los resultados de un simulador hidráulico externo como, por ejemplo, EPANET (Rossman, 2000). El principal inconveniente de este tipo de algoritmos es que la función objetivo precisa ser evaluada muchas veces hasta que se produce la convergencia, lo que supone un elevado esfuerzo computacional. Además, los métodos metaheurísticos no garantizan tampoco la optimalidad de la solución ni ofrecen información sobre cómo de lejos se encuentra el óptimo global.

Jowitt y Germanopoulos (1992) propusieron un método basado en programación lineal para realizar la optimización en tiempo real de los parámetros de operación de los SDA, cuando se dispone de almacenamientos. La programación lineal garantiza el óptimo global y requiere un bajo esfuerzo computacional, siendo así adecuado para la optimización on-line, aunque también se ha utilizado para la optimización off-line (Reca, GarciaManzano, y Martínez 2013; Reca, García-Manzano, y Martínez 2015). La desventaja de este método es el error cometido a causa de la linealización del comportamiento hidráulico. Para mitigar esta limitación, los investigadores han combinado la optimización lineal con otras técnicas, por ejemplo, la búsqueda local (Giacomello et al., 2013) o los algoritmos genéticos (Bou Soler, 2016), de modo que la programación lineal genera una solución inicial, mientras que la segunda técnica se encarga de proporcionar la precisión.

En el caso de sistemas que disponen de almacenamientos, la programación dinámica permite el manejo de modelos hidráulicos más realistas y garantiza la optimalidad de la solución. Sin embargo, la precisión de la solución depende del nivel de discretización de las variables de estado, que son continuas. Una discretización más grosera conducirá a una solución menos precisa, pero el esfuerzo computacional requerido se reducirá. Lansey y Awumah (1994) aplicaron con éxito un método basado en programación dinámica a una optimización de la programación de bombeos en tiempo real mediante el uso de un modelo de regresión para el sistema hidráulico. 


\subsubsection{Reducción del consumo energético en el caso particular de redes de riego}

La sustitución de los regadíos tradicionales por riego localizado ha supuesto un importante incremento en los costes de operación de las redes de riego. El incremento se debe principalmente al consumo energético adicional de los equipos de bombeo para elevar la presión de trabajo hasta el nivel requerido por las subunidades de riego (Rodríguez-Díaz et al., 2011). Además, tanto los crecientes precios de la energía como el cambio en las estructuras tarifarias de los últimos años, son causa a su vez del aumento en los costes del bombeo (Langarita et al., 2017).

Una de las líneas de investigación encaminadas hacia la optimización energética de redes de riego por bombeo directo se ha centrado en mejorar su operación. Concretamente, se ha demostrado que las actuaciones en el ámbito de las demandas permiten generar escenarios de consumo más eficientes energéticamente. Rodríguez Díaz et al. (2009) demostró que una sectorización de la red, en la que se agrupen las demandas siguiendo criterios topográficos, puede llegar a ser hasta un 30\% más eficiente que una red a la demanda si, además, se ajusta la presión de consigna en la estación de bombeo al valor mínimo requerido. Sin embargo, el criterio topográfico no garantiza que el escenario propuesto sea óptimo, ya que no considera factores determinantes como las pérdidas de carga en las tuberías. Jiménez-Bello et al. (2010) plantea el problema de optimización para definir la mejor sectorización de la red que minimice el consumo energético global, a la vez que se garantiza la presión mínima de funcionamiento en todos los hidrantes. Los algoritmos metaheurísticos son los más adecuados para resolver este tipo de problemas, por su naturaleza no lineal y multimodal. Concretamente, en el citado trabajo se eligió un algoritmo genético.

Fernández García et al. (2013) avanzó en esta línea de investigación considerando redes con varios puntos de suministro, y el uso de un algoritmo genético multi-objetivo. Como primer objetivo se propuso minimizar la suma normalizada del coste de bombeo y el déficit de volumen aportado y, como segundo objetivo la suma normalizada de los hidrantes con déficit de presión y el valor de este. No obstante, la determinación de los sectores de riego continuó obteniéndose mediante criterios topográficos.

La sectorización de la red, tal como se plantea en la bibliografía mencionada anteriormente, presenta una serie de oportunidades de mejora. Por una parte, puesto que en distintas parcelas se pueden dar diferentes cultivos, o estos pueden encontrarse en fases 
fenológicas distintas, no se debería establecer a priori un tiempo de riego único para todas las parcelas de un mismo sector, como es característico del riego por turnos. El establecimiento de tiempos de riego ajustados a cada parcela individualmente permitiría aumentar los grados de libertad del problema de optimización, con lo que se podrían alcanzar ahorros energéticos mayores. Esto quedó demostrado en Jiménez-Bello et al. (2011). Por otra parte, se debe tener en cuenta que las necesidades de agua de los cultivos varían a lo largo del año, incluso diariamente en función de las condiciones meteorológicas. Por este motivo, una operación óptima de la red pasa por resolver el problema de optimización en tiempo real, adaptando la programación del tiempo de operación de los hidrantes a las necesidades diarias de agua de los diferentes cultivos.

En esta línea, García et al. (2015) desarrolló una metodología para la optimización de la programación del riego con un horizonte temporal tanto semanal como diario. La metodología se basa en un algoritmo metaheurístico de la familia ACO (Ant Colony Algorithms). Los algoritmos metaheurísticos han demostrado su eficacia para la resolución de este tipo de problemas, dada su versatilidad y sencillez de implementación. Sin embargo, se trata de algoritmos poco eficientes computacionalmente comparados con los algoritmos clásicos (programación lineal, no lineal, etc.), es decir, requieren un elevado número de evaluaciones de la función objetivo hasta obtener una solución cuasi-óptima. Elevados tiempos de computación limitan la aplicabilidad en tiempo real de un optimizador de la programación de riego, en base al cálculo diario de las necesidades hídricas de los cultivos.

Se han propuesto en la literatura varias técnicas para acelerar los algoritmos evolutivos. Existen técnicas basadas en la optimización de las funciones de mutación y cruce (Nia y Alipouri 2009), otras basadas en el uso de modelos sustitutos (Rasheed, Ni, y Vattam 2005), otras basadas en mejorar la diversidad de la población (Jassadapakorn y Chongstitvatana 2011) y las que se basan en aprovechar la computación multihilo (Sinha et al., 2015).

Uno de los principales retos abordados en la presente Tesis ha sido la búsqueda de una mejor eficiencia computacional para resolver el problema de optimización de la programación de riego, en términos generales. Para ello se ha indagado en la aceleración de los métodos de optimización a través de tres estrategias: 
- El replanteamiento de los problemas buscando una dinámica más voraz, es decir, reduciendo la complejidad del problema.

- La mejora de la diversidad poblacional, mediante la introducción de un enfoque multiobjetivo.

- El cálculo multihilo, aprovechando las ventajas de los ordenadores multi procesador actuales.

\subsection{Estructura de la Tesis}

La presente Tesis se ha estructurado en 7 capítulos. En este primer capítulo se ha expuesto el contexto del problema que se pretende tratar, así como una pequeña descripción del estado del arte en la que se resume cómo se ha abordado el tema en trabajos de investigación anteriores.

El segundo capítulo contiene una breve descripción de algunos de los métodos generales de optimización existentes, los cuales se han clasificado utilizando como criterio la propia estrategia que utilizan para la búsqueda de la mejor solución. Esta clasificación será de ayuda para una mejor comprensión de la problemática objeto de la Tesis. Además, en este capítulo se describen las ecuaciones de modelización hidráulica tal como están planteadas y resueltas por el software de simulación EPANET, debido su papel imprescindible en cada una de las metodologías de optimización propuestas.

En el tercer capítulo se trata en profundidad la optimización energética de subsistemas de distribución de agua de riego con inyección directa mediante estaciones de bombeo con, al menos, una bomba de velocidad variable, que funcionan siguiendo una consigna de presión. En el capítulo se expone el método general de optimización que se tomó como punto de partida para la realización de la Tesis, así como una serie de mejoras introducidas sobre este método general. También se describen los casos de estudio que sirvieron para probar la eficacia de las mejoras propuestas.

El cuarto capítulo incluye la descripción de un proceso sistemático para la construcción de las ecuaciones que conforman el problema de optimización energética de subsistemas de transporte de agua, incluyendo la presencia de depósitos, bombas y válvulas, en las cuales se basa en el método de optimización mediante programación lineal propuesto. Se incluye la aplicación del método en un caso de estudio real. 
En el quinto capítulo se presenta un novedoso enfoque para el cálculo de los flujos energéticos que es capaz de ofrecer información detallada acerca del origen y del destino final de las aportaciones energéticas en los sistemas de transporte y distribución de agua. Se presta especial interés a los bombeos como fuentes de energía del sistema hidráulico, ya que son estos los que incurren en un coste para el gestor del SDA. El capítulo contiene también la definición de un método heurístico que, gracias al novedoso modelo de cálculo, permite la optimización energética de subsistemas de transporte de agua en tiempo real, ofreciendo algunas ventajas de las que carecen los métodos basados en algoritmos generales de optimización.

El sexto capítulo contiene la descripción del método aplicado para el filtrado de datos de campo que se ha desarrollado para poder realizar la carga de los modelos hidráulicos de forma adecuada.

Finalmente, el séptimo capítulo contiene las conclusiones generales de este estudio. También se enumeran las aportaciones originales de la Tesis y las publicaciones realizadas en relación con ellas. Se concluye con una breve nota acerca de algunos trabajos que supondrían una continuación de los avances realizados en la Tesis, y que podrían resultar de interés en el ámbito de la optimización energética en tiempo real. 



\section{CAPÍtULO 2.}

\section{HERRAMIENTAS UTILIZADAS}

\subsection{Generalidades de los métodos de optimización}

Un problema de optimización puede definirse como la búsqueda de la solución que maximiza o minimiza una determinada función. La complejidad de los problemas de optimización depende de diversos factores, como puede ser el tamaño del espacio de búsqueda, la no linealidad de la función o la existencia de óptimos locales (multimodalidad).

Los problemas de optimización pueden clasificarse en base a múltiples criterios. Por ejemplo, se pueden diferenciar los problemas de optimización sin restricciones de los que incorporan restricciones que debe cumplir la solución para que se considere factible; también se pueden clasificar en función de la naturaleza de las variables de decisión distinguiendo entre optimización discreta, continua o mixta; o también se puede hacer hincapié en el número de objetivos a satisfacer, usualmente contrapuestos, denominándose problemas multiobjetivo cuando son más de uno (MOO, Multi-Objective Optimisation).

En este apartado se pretende enumerar algunos de los principales métodos de optimización utilizados en la literatura, ofreciendo una visión global de estas técnicas en base a su clasificación en tres categorías: métodos deterministas, métodos heurísticos y métodos metaheurísticos.

\subsubsection{Métodos deterministas}

Los métodos de optimización deterministas o matemáticos utilizan de algún modo las propiedades analíticas de las propias ecuaciones que gobiernan el sistema a optimizar para generar una secuencia de nodos que converjan a una solución exacta, la cual responde 
a un óptimo local o global (Lin, Tsai, y Yu 2012). Entre los métodos deterministas cabe citar la programación lineal, la programación cuadrática y la programación no lineal.

La programación lineal se aplica a problemas en los que tanto la función objetivo como las restricciones pueden expresarse como funciones lineales dependientes de las variables de decisión. El algoritmo más utilizado para la resolución de este tipo de problemas es el método Simplex. El caso particular de un problema lineal en el que las variables de decisión son continuas ofrece dos importantes ventajas: el algoritmo garantiza el óptimo global, siempre que este exista, y además presenta una gran eficiencia computacional que permite resolver problemas complejos de gran tamaño en un tiempo muy reducido.

La programación cuadrática es un caso particular de programación no lineal en el que la función objetivo puede expresarse como una función cuadrática de las variables de decisión, mientras que las restricciones se mantienen como funciones lineales. En este caso, los algoritmos utilizados para la búsqueda del mínimo también garantizan que se alcanza la solución óptima, si existe (Arora, 2004).

En el caso de la programación no lineal, ni la función objetivo ni las restricciones son lineales. Todavía dentro de esta categoría se encuentra la optimización convexa, un tipo de problema para el que existen métodos capaces de calcular el óptimo global. La programación cuadrática sería un caso particular de optimización convexa. Sin embargo, el caso más general responde a problemas no convexos (por ejemplo, multimodales), para los que los métodos deterministas existentes no garantizan que se alcance el óptimo global.

La mayoría de los métodos deterministas descritos se formularon inicialmente para la optimización de problemas en los que las variables de decisión se suponen continuas en todo el dominio de definición, aunque ello no es condición necesaria. Sin embargo, la inclusión de variables discretas en la formulación añade una notable complejidad a los problemas. Aunque en el caso de la programación lineal existen métodos efectivos, como el método de "Ramificación y Poda" (Branch and Bound) que son capaces de llegar a la solución óptima, los tiempos de cálculo pueden dispararse cuando el número de variables discretas es elevado. Para otros métodos no puede garantizarse la consecución del óptimo cuando intervienen variables discretas. 


\subsubsection{Métodos heurísticos}

A medida que la complejidad de los problemas de optimización aumenta (combinación de problemas no lineales, problemas de variable discreta y problemas multimodales) surgen enfoques alternativos dirigidos a encontrar de manera eficiente una solución aproximada, que aunque no necesariamente coincida con el óptimo global, esté dentro de un límite aceptable, buscando un compromiso entre precisión y tiempo de cálculo (El-Omari, 2020). Los métodos heurísticos siguen una regla (heurística) que debe definirse 'ad hoc' para cada problema de optimización concreto, la cual debe conducir a encontrar una aproximación a la solución del problema, utilizando normalmente una estrategia voraz. De este modo, siempre a condición de que la heurística sea acertada, se pueden alcanzar buenas soluciones con un esfuerzo computacional mínimo.

En consecuencia, los métodos heurísticos presentan una dependencia con el problema a resolver. Por lo general deben adaptarse a la naturaleza del problema en cuestión, tratando de aprovechar al máximo las particularidades del mismo. Sin embargo, estos métodos no ofrecen ninguna garantía de cercanía al óptimo de la solución proporcionada y, debido a la estrategia voraz, por lo general no logran obtener la solución óptima global. Aun así, estas técnicas son de gran utilidad en ámbitos en los que se precisa un cierto grado de inmediatez en el cálculo de la solución y se tolera un margen de desviación respecto a la solución óptima, como por ejemplo en la generación de rutas para vehículos (Pisinger y Ropke 2007).

\subsubsection{Métodos metaheurísticos}

Los métodos metaheurísticos, por el contrario, son técnicas que no dependen del problema a resolver, no aprovechan ninguna especificidad del problema y, por lo tanto, pueden ser vistos como cajas negras. Así, un mismo método puede ser de aplicabilidad en múltiples problemas, es más, suelen resultar fácilmente adaptables a las particularidades de cualquier sistema. En general, son poco voraces, incluso pueden asumir empeoramientos de la solución lo que les permite explorar más a fondo el espacio de soluciones y, por lo tanto, obtener una mayor garantía de que la solución encontrada no responde a un estancamiento en un óptimo local.

Los métodos metaheurísticos, de modo similar a los heurísticos, definen una serie de reglas que conducen a la obtención de mejores soluciones progresivamente. A diferencia de los heurísticos, en este caso las reglas no se extraen a partir del conocimiento del problema, 
sino que se basan en la idea de combinar una exploración eficiente del espacio de soluciones con la búsqueda local en el entorno de las mejores soluciones encontradas. Muchos de estos métodos se inspiran en comportamientos que pueden observarse en la naturaleza. Por otra parte, aunque los métodos metaheurísticos son independientes del problema, es necesario hacer algunos ajustes de sus parámetros intrínsecos con el fin de adaptar la técnica al problema en cuestión.

De entre los métodos metaheurísticos más empleados pueden nombrarse los siguientes:

- Algoritmos genéticos

- Búsqueda tabú

- Recocido simulado

- Optimización por enjambre de partículas

- Optimización por colonia de hormigas

- Colonia artificial de abejas

Por su importancia en la bibliografía y por su utilización en la presente Tesis, se explica brevemente a continuación el método general de optimización por algoritmos genéticos, que históricamente fue del punto de partida de todos ellos.

\subsubsection{Fundamentos de los algoritmos genéticos}

Siguiendo las propuestas predominantes en los principales trabajos de investigación en el ámbito de la optimización energética de redes de riego, en la presente Tesis se ha profundizado en la explotación de los métodos metaheurísticos, concretamente en los algoritmos genéticos, cuya eficacia en la resolución de problemas similares al planteado aquí ya ha sido ampliamente demostrada.

Los algoritmos genéticos son métodos evolutivos de optimización basados en el manejo de un conjunto de soluciones posibles (población). Las soluciones se clasifican en función de su bondad a través de un indicador que se calcula a partir de una función objetivo. Los algoritmos genéticos, así como otros métodos de optimización (colonia de hormigas, enjambre de partículas, recocido simulado, etc.), están inspirados en los procesos evolutivos de la naturaleza, lo que podría entenderse como el grado de efectividad de un organismo para competir por unos determinados recursos. Los individuos con un mayor grado de 
adaptación tendrán una mayor probabilidad de reproducirse, replicando así parte de su material genético en la siguiente generación.

El funcionamiento básico de un Algoritmo Genético se puede resumir en los pasos que se explican a continuación.

\section{Definición de las variables del problema.}

Cada problema en particular tendrá unas variables de decisión que pueden representarse mediante dígitos binarios, números enteros o números reales. En base al tipo de variables a utilizar para resolver el problema, el algoritmo genético deberá ejecutar las funciones internas de mutación y cruzamiento de distinta forma. El vector que contiene los valores de todas las variables del problema se denomina cromosoma, y representa una solución al mismo.

\section{Generación de una población inicial.}

Una vez definido el tipo de variables a utilizar, así como su número, se crea una población inicial que sirve como punto de partida del algoritmo genético. Normalmente, esta población inicial se genera de manera aleatoria. Pero hay casos en los que puede resultar útil la introducción de algún individuo con ciertas características, si se conocen o intuyen de antemano, con el objetivo de lograr una convergencia más rápida.

\section{Evaluación de aptitud}

Se debe declarar al menos una función objetivo a través de la cual cada individuo es evaluado para conocer su grado de adaptabilidad en el entorno en consideración. En caso de que todas las posibles combinaciones existentes en el espacio de soluciones fuesen factibles, la definición de la función objetivo sería relativamente sencilla. Por ejemplo, en el ámbito de la optimización energética, la función objetivo podría calcular el consumo energético por unidad de volumen suministrado de cada solución. Sin embargo, esta tarea puede resultar delicada debido a la existencia de soluciones no factibles. Ante esta situación, se han propuesto diversos procedimientos a seguir.

El primero de ellos sería el descarte directo de las soluciones no factibles y su restitución por un nuevo individuo obtenido igualmente mediante los mecanismos de cruzamiento y mutación. El segundo planteamiento consiste en la modificación de los cromosomas que no verifican la factibilidad, actuando únicamente sobre los genes (variables) causantes de la no factibilidad, si es que se pueden identificar. 
Por último, para tener en cuenta las restricciones el procedimiento más utilizado consiste en penalizar la función objetivo. La idea general consiste en introducir un factor de penalización que multiplique (o divida, en caso de que el objetivo sea una maximización) al valor de la función objetivo del individuo en base a las restricciones que dicho individuo viola. El valor del factor puede tener en cuenta el número de restricciones violadas. Otra alternativa es incorporar un término aditivo (o sustractivo si se trata de una maximización) debidamente ponderado por cada una de las restricciones violadas.

\section{Selección de individuos}

De la población existente de cromosomas en un momento dado se requiere realizar un proceso de selección. Los individuos seleccionados son usados después en los procesos de cruzamiento y mutación para producir una nueva población, que contendrá soluciones al menos tan buenas como la generación anterior. La solución más adoptada es mantener las mejores soluciones (elitismo) y completar las faltantes mediante los procesos de cruzamiento y mutación, manteniendo así siempre el mismo tamaño de la población.

\section{Cruzamiento}

Una vez los progenitores han sido seleccionados, se produce el cruzamiento. El operador de cruce para un punto selecciona aleatoriamente un punto de corte del cromosoma, a partir del cual los progenitores intercambian las subcadenas ubicadas a continuación de dicho punto. Existen también operadores de cruce basados en más de un punto de corte. Estos operadores pueden mejorar el comportamiento del algoritmo al explorar de una manera más eficiente el espacio de soluciones.

\section{Mutación}

Es el operador que aleatoriamente cambia uno o más de los genes de los cromosomas. La mutación permite la exploración de las soluciones vecinas, resultando de gran utilidad sobre todo en las últimas etapas de convergencia, cuando el grado de exploración del espacio de soluciones ya es elevado.

\section{Finalización}

Los procesos de selección, cruzamiento y mutación se van iterando, creando sucesivas generaciones. En cada nueva generación hay siempre un cromosoma que es igual o mejor que los de la población anterior. Los criterios para finalizar las iteraciones son varios. 
Como primer criterio, se detiene el proceso cuando las sucesivas generaciones no mejoran la función objetivo. Se entiende entonces que el óptimo global se ha alcanzado, aunque cabe la posibilidad de que sea un óptimo local al que se ha convergido prematuramente. Este criterio determina cuándo el proceso genético termina de evolucionar.

Pero también se puede finalizar el proceso fijando el número máximo de generaciones a ensayar, o estableciendo un tiempo máximo de cálculo. También se puede fijar un número mínimo de generaciones para evitar caer prematuramente en un óptimo local.

\subsection{Software de simulación hidráulica: EPANET}

EPANET es un programa de ordenador para el cálculo de redes hidráulicas a presión. Realiza tanto simulaciones del comportamiento hidráulico (presiones, caudales) como de calidad del agua (concentraciones, reacciones...). EPANET utiliza modelos cuasi-estáticos o en periodo extendido. Estos modelos simulan el comportamiento a lo largo del tiempo, pero sin tener en cuenta el efecto de los transitorios causados por las maniobras de elementos como válvulas o bombas, ni tampoco las oscilaciones provocadas por la inercia de la columna de agua (Iglesias-Rey, Martínez-Solano, y Ribelles-Aquilar 2017).

\subsubsection{Modelado matemático}

EPANET realiza el cálculo hidráulico mediante el Método del Gradiente descrito por (Todini y Pilati, 1988). Este método se basa en las siguientes ecuaciones:

$$
\begin{gathered}
H_{i}-H_{j}=h_{i j}=r Q_{i j}^{n}+m Q_{i j}^{2} \\
\sum_{j} Q_{i j}-D_{i}=0
\end{gathered}
$$

- $\quad H_{i}$ es la altura en el nudo $i$.

- $\quad h_{i j}$ son las pérdidas en la línea entre el nudo $i$ y el nudo $j$.

- $r$ es el coeficiente de resistencia.

- $\quad Q_{i, j}$ es el caudal que circula por la línea entre el nudo $i$ y el nudo $j$.

- $n$ el exponente del caudal.

- $m$ es el coeficiente de pérdidas menores.

- $\quad D_{i}$ es la demanda de caudal en el nudo $i$. 
Dadas las alturas piezométricas en algunos de los nudos del modelo, se ha de encontrar la solución para las incógnitas $H_{i}$ y $Q_{i, j}$ que cumplan las identidades (1) y (2). La resolución del Método del Gradiente se inicia suponiendo un determinado valor para los caudales de línea, sin necesidad de que estos caudales cumplan inicialmente las ecuaciones de continuidad. Dichos caudales se utilizan para determinar el punto de linealización inicial de las curvas de comportamiento de cada elemento. A continuación, se realizan una serie de cálculos iterativos en los que en cada iteración se actualizan las alturas piezométricas no prefijadas mediante la resolución del siguiente sistema de ecuaciones lineales:

$$
A \cdot H=F
$$

resultante del proceso de linealización, donde:

$A$ es la matriz Jacobiana cuyos elementos en la diagonal son:

$$
A_{i i}=\sum_{j} p_{i j}
$$

mientras que el resto de los elementos de la matriz vienen dados por:

$$
A_{i j}=-p_{i j}
$$

siendo $p_{i j}$ es la inversa de la derivada parcial respecto al caudal de la pérdida de carga en la línea que une los nudos $i$ y $j$ :

$$
p_{i j}=\frac{1}{n r\left|Q_{i j}\right|^{n-1}+2 m\left|Q_{i j}\right|}
$$

El término $H$ es el vector que contiene las incógnitas del sistema, que corresponden a las alturas piezométricas en los nudos en que no está prefijada.

Cada término de la derecha $F_{i}$ corresponde al desequilibrio del caudal neto en cada nudo más un factor de corrección de caudal, esto es:

$$
F_{i}=\left(\sum_{j} Q_{i j}-D_{i}\right)+\sum_{j} y_{i j}+\sum_{f} p_{i f} H_{f}
$$

siendo el factor de corrección de caudal $y_{i j}$ :

$$
y_{i j}=p_{i j}\left(r\left|Q_{i j}\right|^{n}+m\left|Q_{i j}\right|^{2}\right) \operatorname{sgn}\left(Q_{i j}\right)
$$

y $H_{f}$ las alturas piezométricas prefijadas en los nudos. 
Una vez calculadas las alturas mediante la Ecuación (3), se determinan los caudales mediante la siguiente expresión:

$$
Q_{i j}=Q_{i j}-\left(y_{i j}-p_{i j}\left(H_{i}-H_{j}\right)\right)
$$

El cálculo de los nuevos caudales a partir de esta expresión, en lugar de despejar de la Ecuación (1), es lo que confiere al método del gradiente la rapidez de convergencia que le caracteriza.

El sistema lineal de ecuaciones representado por la Ecuación (3) se resuelve utilizando un método de matrices dispersas basado en la reordenación previa de los nudos, que en la versión 2.2 ha sido optimizado con el método del Grado Mínimo Múltiple (MMD) reduciendo así el tiempo de cálculo en la primera iteración hasta en un $55 \%$. Tras la reordenación se lleva a cabo una factorización simbólica, de manera que solo los elementos no nulos de $A$ se calculan y guardan en memoria.

Para la primera iteración se establece en cada tramo un caudal inicial correspondiente a una velocidad de $1 \mathrm{ft} / \mathrm{s}$. En caso de ser una bomba, el caudal inicial se establece como el caudal de diseño, o el caudal central de la serie de puntos que identifican su curva característica. Todos los cálculos se llevan a cabo internamente con la altura en pies y el caudal en pies cúbicos por segundo, si bien a nivel de usuario se puede trabajar con unidades en el sistema internacional SI o en el sistema anglosajón US.

El coeficiente de resistencia de una tubería $(r)$ se calcula como se describe en la Tabla 1, en función de la fórmula utilizada para el cálculo de las pérdidas.

Tabla 1. Cálculo de pérdidas en tuberías (pérdidas en pies y caudal en cfs).

\begin{tabular}{ccc}
\hline Fórmula & Coef. De Resistencia (r) & Exponente de Caudal (n) \\
\hline Hazen-Williams & $4,727 \mathrm{C}^{-1,852} \mathrm{~d}^{-4,871} \mathrm{~L}$ & 1,852 \\
Darcy-Weisbach & $0,0252 \mathrm{f}(\varepsilon, \mathrm{d}, \mathrm{q}) \mathrm{d}^{-5} \mathrm{~L}$ & 2 \\
Chezy-Manning & $4,66 \mathrm{n}^{2} \mathrm{~d}^{-5,33} \mathrm{~L}$ & 2 \\
\hline
\end{tabular}

siendo

- $\mathrm{C}=$ coeficiente de rugosidad de Hazen-Williams

- $\varepsilon=$ coeficiente de rugosidad de Darcy-Weisbach

- $\mathrm{f}=$ factor de fricción (depende de $\varepsilon, \mathrm{d}$ y q)

- $\mathrm{n}=$ coeficiente de rugosidad de Manning 
- $\mathrm{d}$ = diámetro de la tubería (ft)

- $\quad \mathrm{L}=$ longitud de la tubería (ft)

- $\mathrm{q}=$ caudal $(\mathrm{cfs})$

Para la ecuación de pérdidas de Darcy-Weisbach, el factor de fricción f se calcula en función del número de Reynolds (Re). En el rango $2000<\operatorname{Re}<4000$ se utiliza una fórmula de interpolación cúbica en entre la fórmula de Hagen-Poiseuille correspondiente al régimen laminar y la aproximación de Swamee y Jain para el régimen turbulento, garantizando así la continuidad del valor de $\mathrm{f}$ y de su derivada en todo el rango de valores de Re.

Por último, el coeficiente de pérdidas menores $K$, adimensional cuando se expresa en función de la altura dinámica, se convierte en el coeficiente $m$ que multiplica al caudal a partir de la siguiente relación:

$$
m=\frac{0,02517 K}{d^{4}}
$$

\subsubsection{Toolkit de EPANET}

El Módulo de Herramientas (Toolkit) para Programadores de EPANET es una librería dinámica programada en $\mathrm{C}$, que contiene una serie de funciones relacionadas con la simulación de modelos hidráulicos que pueden ser invocadas desde otras aplicaciones. La Toolkit puede conectarse con cualquier lenguaje de programación que permita la llamada a una DLL de Windows. Así, esta herramienta supone una gran capacidad de personalización, permitiendo a los desarrolladores programar múltiples y variadas rutinas para alcanzar objetivos concretos mediante el uso de la simulación hidráulica como paso intermedio.

En su versión 2.2, el Módulo de Herramientas permite, además de obtener los resultados de la simulación hidráulica, construir desde cero, modificar y eliminar los diferentes elementos de una red hidráulica, tanto físicos como no físicos (curvas de modulación, leyes de control, etc.), desde un entorno de programación, sin necesidad de utilizar como apoyo la interfaz de usuario de EPANET. Esta flexibilidad, no disponible en la versión 2.0 del Módulo de Herramientas, ofrece una enorme potencia para el desarrollo de aplicaciones pensadas como sistemas de soporte a la toma de decisiones en cuanto al diseño y optimización de nuevas infraestructuras.

Además, en la versión 2.2 se solventa finalmente la limitación que presentaba la versión 2.0 en cuanto al manejo de múltiples escenarios. El Módulo de Herramientas 2.0 no 
permitía el manejo de más de una red simultáneamente, limitando o dificultando en gran medida la optimización computacional de los algoritmos a través de la paralelización.

\subsubsection{Modelización hidráulica para la optimización energética}

Todas las metodologías que se describen en la presente Tesis se apoyan directa o indirectamente en la utilización de un modelo hidráulico para simular cualquier escenario propuesto. No es objeto de la Tesis profundizar en todos los aspectos relacionados con la generación, carga y calibración de los modelos hidráulicos, aunque necesariamente están presentes en todos los desarrollos. En este apartado se tratará de esbozar las principales ideas que se han tenido en cuenta.

En primer lugar, en todos los casos de estudio se ha contado con un modelo hidráulico previamente construido, el cual se asume que reproduce fielmente la realidad. La validación del modelo no forma parte de los objetivos de esta Tesis. En cambio, sí se aborda la carga del modelo a partir de los datos disponibles procedentes de la sensorización de la red con el objetivo de reproducir escenarios pasados con el mayor grado de precisión posible.

En el ámbito de la presente Tesis, se define el proceso de "carga" del modelo como:

- La determinación de las curvas de modulación o patrones de demanda correspondientes a cada nudo o conjunto de nudos de consumo a lo largo del periodo de simulación.

- La generación de las leyes de control de los elementos de regulación (válvulas y bombas) que reproducen las consignas de operación en función del tiempo o del estado hidráulico de otros elementos de la red.

En los casos de estudio correspondientes a redes de riego, la asignación de demandas se ha realizado a partir de las lecturas de los contadores de cada toma de riego. En todos los casos, se ha observado una buena coincidencia con el caudal total inyectado. Así, en estos casos no ha sido necesario plantearse una estrategia de asignación de fugas latentes en los nudos de la red para hacer coincidir los caudales medidos con los simulados.

En cambio, en las experiencias con redes de distribución de agua potable, se ha observado una discrepancia entre la suma del caudal consumido y del inyectado, debido a las pérdidas, tanto reales como aparentes. En estos casos, puesto que el análisis del rendimiento hidráulico excede el alcance de la Tesis, se ha optado por realizar un reparto del 
agua no registrada entre los nudos de consumo, de forma proporcional al propio consumo de cada nudo.

En esta línea, los modelos hidráulicos para la optimización energética deben presentar un grado de calibración suficientemente preciso para que los resultados obtenidos sean fiables. Solo así se garantizará que los escenarios óptimos cumplen las restricciones propuestas, y que el consumo energético calculado se ajusta a la realidad. Los modelos hidráulicos se calibran para un conjunto de escenarios observados. En esta Tesis se supone que dichos modelos reproducirán también la realidad cuando sean sometidos a otros escenarios propuestos.

Finalmente, aunque la versión 2.2 de EPANET cuenta con la opción de que las demandas sean dependientes de las presiones, en todos los casos de aplicación presentados se han utilizado modelos conducidos por demandas, es decir, para la resolución del sistema de ecuaciones hidráulicas se asume que los caudales demandados se han de satisfacer completamente, aunque las presiones resultantes en los nudos sean insuficientes (incluso presiones negativas). Los modelos conducidos por demandas no ofrecerían resultados correctos o realistas en la simulación de escenarios de emergencia (roturas, fugas, demandas excesivas, etc.), pero en el contexto de la Tesis son adecuados, ya que en todos los casos se trata de garantizar la presión de servicio y, en consecuencia, las soluciones con presiones insuficientes quedan descartadas. 


\section{CAPÍtULLO 3.}

\section{OPTIMIZACIÓN ENERGÉTICA DE REDES DE RIEGO CON BOMBEO DIRECTO}

\subsection{Introducción a las particularidades de las redes de riego}

Los sistemas de distribución de agua a presión en el ámbito de la agricultura comparten parte de las características morfológicas que identifican a los sistemas de abastecimiento urbano. Puede tomarse como generalización que en ambos sistemas el agua discurre por conducciones a presión, que pueden existir almacenamientos intermedios, elementos que aportan energía al fluido (bombas hidráulicas) y elementos que disipan la energía del fluido (válvulas hidráulicas).

Debido a las semejanzas que presentan los sistemas de riego y los sistemas de abastecimiento urbano, algunas de las herramientas de las que se dispone y que conforman las piezas con las que se construyen las metodologías de optimización planteadas en la presente Tesis, resultan comunes a ambos campos. Concretamente, tanto los sistemas de riego como los urbanos, en tanto en cuanto se trata de sistemas de distribución de agua a presión, comparten los mismos supuestos, condiciones y procedimientos en su modelización matemática. Por lo tanto, los métodos propuestos en la Tesis para la optimización energética de redes de riego se apoyan también en la herramienta de modelización hidráulica EPANET.

Sin embargo, se pueden vislumbrar algunas diferencias, que tienen mayor o menor impacto en la viabilidad de las metodologías de optimización a aplicar. Entre ellas, cabe mencionar las siguientes: 
- Las redes de riego suelen presentar una morfología ramificada o con un grado de mallado muy inferior a las redes de abastecimiento urbano.

- El dimensionado de las tuberías en redes de riego suele ser más ajustado, mientras que en sistemas urbanos tiende a ser más holgado.

- En los sistemas urbanos se da con poca frecuencia una configuración de inyección directa a red. Usualmente se bombea previamente a depósito para garantizar el suministro.

- En los sistemas urbanos se tiene muy poca o nula capacidad para influir sobre las demandas de los usuarios, mientras que los sistemas de riego permiten un cierto grado de organización de las demandas desde un sistema centralizado, como ocurre en con el riego por turnos o el riego programado.

Todas estas diferencias se fundamentan en una causa común, que es la necesidad ineludible de maximizar la seguridad en el suministro en el caso de las redes urbanas, garantizando en todo momento los caudales demandados en cada punto en unas condiciones de presión preestablecidas. Por el contrario, en las redes de riego este aspecto no sería tan crítico, considerándose aceptable mantener una cierta flexibilidad en los instantes concretos en los que se satisface la demanda de cada parcela.

Estas características diferenciadoras de las redes agrícolas frente a las urbanas tienen una influencia determinante en los posibles planteamientos del problema de optimización energética. Esto no quiere decir que ninguno de los métodos de optimización propuestos en el ámbito de las redes urbanas sea de aplicabilidad en redes de riego, o viceversa. Como ejemplo, el método de optimización lineal sería aplicable a sistemas en los que haya un bombeo a depósito y una posterior distribución por gravedad a las parcelas, independientemente de la posibilidad de actuar o no sobre las demandas.

El hecho de que muchos sistemas de riego a presión funcionen mediante el bombeo directo a red, aspirando el agua desde una balsa, introduce un nuevo reto a la hora de definir el método de optimización más adecuado para reducir el consumo energético de las bombas. Estos sistemas normalmente cuentan con bombas de velocidad variable (BVV) que, junto con automatismos de control de la velocidad de giro, son capaces de mantener una presión de consigna prefijada en la impulsión. La implicación que esto tiene en el contexto de los métodos de optimización es que la modelización de las estaciones de bombeo se vuelve más compleja, impidiendo asunciones simplificativas como las que se describirán en el 
CAPÍTULO 4. No obstante, debe remarcarse que, desde un punto de vista más general, las inyecciones directas a red con bombas que dispongan de variador de velocidad conforman un escenario mediante el cual, en teoría, se pueden alcanzar mayores ahorros energéticos que con los bombeos a depósito al poder ajustar la presión de impulsión, directamente relacionada con la energía que consumen las bombas, a la mínima estrictamente necesaria para vencer los desniveles topográficos, compensar las pérdidas por fricción en las tuberías y entregar el agua en cada punto de suministro a una presión mínima de servicio.

Por otra parte, dada la relativa flexibilidad a la hora de distribuir temporalmente las demandas a lo largo de cada jornada, los sistemas de riego brindan la oportunidad de incluir, en el conjunto de variables de decisión del problema, la determinación de las franjas horarias más convenientes a las que asignar cada una de las demandas. Entiéndase que cada demanda vendría determinada, bajo criterios agronómicos, por el volumen total de agua a suministrar en un periodo de tiempo determinado (habitualmente será una jornada de riego), es decir, que el volumen se podría suministrar libremente en cualquier franja de tiempo comprendida entre el inicio y el final de la jornada de riego. El hecho de añadir este grado de libertad adicional al problema de optimización presenta la ventaja de aumentar el margen potencial de mejora de la eficiencia energética del sistema, a la vez que se añade complejidad a la metodología de resolución del problema.

Tal como se ha visto en la revisión bibliográfica, estas nuevas complejidades han conducido a los investigadores a explotar las capacidades que ofrecen los métodos metaheurísticos de optimización. Estos métodos de optimización se caracterizan por la búsqueda de la solución óptima mediante procedimientos iterativos que son guiados por una heurística, entendida como una regla general de la que se espera que sea capaz de generar nuevas soluciones potencialmente mejores que las de partida, y que exploran de forma eficiente el espacio de soluciones para encontrar una solución aceptable, aunque sin garantía de que se alcance el óptimo global.

Este tipo de algoritmos son adecuados para la resolución de problemas complejos, no lineales, multimodales (esto es, con múltiples mínimos o máximos locales), donde los métodos tradicionales de optimización matemática no son de aplicación o no son eficaces por quedarse estancados en mínimos locales. Además, son muy versátiles, adaptándose de manera relativamente sencilla a cualquier tipo de problema, y fáciles de implementar. 
En concreto, la aplicabilidad de los métodos de programación lineal y cuadrática sería muy limitada a la hora de modelizar el comportamiento de las estaciones de bombeo, así como las pérdidas por fricción en las tuberías. Las hipótesis simplificativas conducirían probablemente a errores en el metamodelo de un orden de magnitud excesivo en referencia a las potenciales mejoras en la eficiencia energética. Por su parte, los métodos de programación no lineal basados en el cálculo de gradientes convergen con facilidad a óptimos locales cuando el problema a solucionar es multimodal.

No obstante, en trabajos futuros se pretende profundizar en el uso de programación lineal en la resolución del problema de optimización energética en sistemas de inyección directa a red y con posibilidad de actuar sobre las demandas. Se espera poder acotar las características que necesariamente han de presentar los sistemas de distribución para que las simplificaciones tengan un impacto aceptable en las soluciones.

\subsection{Metodología general de optimización energética en redes de riego}

La metodología que se expone en este apartado parte de la propuesta realizada por (Jiménez-Bello et al., 2010), que ha supuesto un punto de partida para las aportaciones derivadas del presente capítulo. No obstante, en esta Tesis se va a plantear una formulación más general, con vistas a extender su aplicabilidad al mayor número de casuísticas y configuraciones.

\subsubsection{Definición de una red de riego, con miras a su optimización}

Se considera una red de riego como la infraestructura necesaria para distribuir el agua requerida por los cultivos desde uno o varios puntos de suministro hasta las tomas de cada parcela. Se entiende que la finalidad de este tipo de redes es exclusiva para riego, y que se dispone de automatismos para controlar todos los consumos.

De entre los elementos que conforman las redes de riego, se detallan a continuación aquellos estrictamente necesarios que intervienen en la formulación del problema de optimización, es decir, los componentes sobre los que se puede actuar en el proceso de optimización.

\subsubsection{Fuentes de suministro}

Se trata del origen del agua que alimenta el sistema hidráulico a optimizar. Puede tratarse de un pozo, un azud, un embalse, una balsa de regulación, etc. En el modelo 
hidráulico de EPANET se representa mediante el elemento "embalse". Este elemento de EPANET se caracteriza por constituir una fuente ilimitada de agua y, en principio, con una altura piezométrica constante. Esta característica puede acarrear un pequeño error de modelización si en realidad la altura piezométrica de la fuente de suministro varía. En este caso, dado que EPANET permite modular la altura de un embalse con el tiempo, podría simularse con exactitud, siempre sujetos a la fiabilidad de su predicción durante el periodo de simulación.

En general, en los casos de estudio abordados a lo largo del desarrollo de la Tesis, la oscilación de la lámina libre de los puntos de suministro se ha mantenido dentro de unos límites aceptables y no se ha llegado a aplicar una modulación de su altura piezométrica. No obstante, es una opción a contemplar en el caso más general.

\subsubsection{Estaciones de bombeo}

La configuración aceptada en la metodología propuesta para las estaciones de bombeo consiste en un conjunto de bombas en paralelo en el que, al menos, una de ellas disponga de variador de frecuencia con el objetivo de permitir un control automático capaz de mantener una presión de impulsión constante e independiente del caudal demandado. Se trata de una configuración muy extendida hoy en día y que ofrece los mayores ahorros energéticos potenciales. Pero su modelación en EPANET presenta limitaciones.

En efecto, EPANET no permite la simulación de una estación de bombeo como un conjunto de elementos coordinados por un controlador local, sino que maneja las bombas individualmente, es decir, no ofrece la posibilidad de forzar el punto de funcionamiento de cada bomba a partir de las reglas de control de la estación de bombeo. Por este motivo, los puntos de funcionamiento deben calcularse mediante un proceso externo a EPANET.

Además, como dificultad añadida, debe remarcarse la amplia variedad de configuraciones y sistemas de control que pueden darse en la práctica. Desarrollar una aplicación general capaz de simular el comportamiento de cualquier estación de bombeo, compuesta de $n$ bombas, algunas de velocidad fija y otras de velocidad variable, y no todas iguales, funcionando bajo el control de un automatismo local propio de cada fabricante e instalación, resultaría una tarea muy compleja. Por ello, en los casos de estudio utilizados para validar las metodologías de optimización propuestas, se ha programado un algoritmo individualizado para cada caso. 
Finalmente, para que la simulación en EPANET sea capaz de reproducir el comportamiento de la estación de bombeo bajo una consigna de presión fija a la salida, se ha propuesto la adición de una válvula reductora de presión ficticia a la salida de la estación de bombeo, cuya consigna de presión coincida con la consigna real fijada para la estación de bombeo. Por su parte, se debe imponer el estado de marcha para todas las bombas de la estación a lo largo del periodo de simulación. Con esta estrategia se consigue simular las condiciones de caudal y presión que se darán en la realidad.

Sin embargo, puesto que la válvula ficticia introduce unas pérdidas a su vez ficticias, el cálculo del consumo energético de las bombas en cada situación se determina mediante un algoritmo aparte, como ya se ha comentado.

\subsubsection{Depósitos}

En caso de existir depósitos intermedios en la red de distribución, se incluirán en el modelo de EPANET mediante sus características físicas (cota de solera, diámetro y niveles máximo y mínimo). La metodología de optimización que se describe no considera la estrategia de llenado del depósito como una variable más del problema de optimización, sino que únicamente se reproducen las consignas de control previstas con el objetivo de simular con la máxima exactitud posible la altura piezométrica disponible en cada instante, así como la potencia consumida por las bombas.

\subsubsection{Tomas}

Se han denominado como "tomas" a los puntos independientes de entrega de agua a la parcela. Estas tomas pueden estar agrupadas en un hidrante multiusuario, que normalmente contiene otros elementos como filtros, contadores y válvulas. A efectos de optimización, se entenderá por toma a cada punto de consumo sobre el que se pueda determinar su apertura y cierre de manera independiente.

\subsubsection{Planteamiento del problema base de optimización}

El problema de optimización a resolver, aunque se pretende que tenga la mayor generalidad posible, se basa en una serie de supuestos que a continuación se enumeran.

\subsubsection{Jornada de riego}

Es el marco temporal dentro del cual se pueden mover las variables de decisión contempladas en el problema de optimización, el cual se conoce también como la jornada 
de riego. La duración de la jornada de riego es variable, pero ha de ser impuesta previamente, y ha de coincidir con el periodo de tiempo máximo en el que se han de satisfacer una serie de demandas o peticiones de riego requeridas. En general, la jornada de riego tendrá una duración igual o inferior a 24 horas.

\subsubsection{Condición de tiempo real}

Cada jornada de riego conforma un problema de optimización a resolver, de modo que el tiempo de computación requerido por el algoritmo debe permanecer por debajo de un umbral aceptable, si se pretende utilizar la herramienta en la toma de decisiones a nivel diario. La condición de "tiempo real" en este contexto debe entenderse como la capacidad operativa de generar con una frecuencia diaria las consignas de operación óptimas a ejecutar durante la jornada de riego siguiente. Cabe remarcar que, para considerar operativa la herramienta, un tiempo de computación superior a 60 minutos no sería admisible, pues no permitiría a un usuario el análisis de varios escenarios calculados bajo distintas hipótesis. El objetivo ideal en tiempo de computación sería alcanzar una eficiencia similar a la que presentan los métodos lineales, cuyos tiempos de cálculo se pueden llegar a reducirse hasta el orden de décimas de segundo. Esta capacidad permitiría una experiencia de usuario óptima y facilitaría la introducción de este tipo de herramientas como sistema de soporte a la decisión en la gestión de comunidades de regantes. Sin embargo, como se verá más adelante, alcanzar una eficiencia computacional de esta magnitud, cuando se aplican metodologías de optimización basadas en algoritmos metaheurísticos o evolutivos, supone un gran reto.

\subsubsection{Peticiones de los usuarios}

El modus operandi que aquí se propone para la optimización energética en tiempo real de redes de riego parte de un conjunto de peticiones de agua realizadas por parte de los usuarios para la siguiente jornada de riego. El propio agricultor estima, bien mediante criterios agronómicos o criterios personales, la cantidad de agua necesaria para el riego de su parcela. De manera rigurosa, esta cantidad debería coincidir con aquella necesaria para compensar las pérdidas por evapotranspiración, y determina las necesidades de agua del cultivo. La necesidad de riego básicamente representa la diferencia entre la necesidad de agua del cultivo y la precipitación efectiva. La evapotranspiración, por su parte, depende de cantidad de factores, entre ellos el tipo de cultivo, así como su fase de crecimiento, y las variables meteorológicas como temperatura, grado de humedad, radiación solar o velocidad del viento. 
Una de las hipótesis de las que se parte para la modelización hidráulica es que el caudal suministrado por cada toma es constante y conocido. A pesar de que en un sentido estricto el caudal entregado por una toma depende de la presión disponible en el hidrante, el uso de goteros autocompensantes para el riego de las parcelas hace que las fluctuaciones de caudal puedan despreciarse siempre que la presión de entrega esté dentro de los límites recomendados por el fabricante de los goteros. Así pues, las peticiones de agua se convierten de hecho en peticiones de tiempo de riego dividiendo el volumen a suministrar por el caudal medio de la toma. El algoritmo de optimización debe, pues, satisfacer completamente las peticiones de agua ubicando cada evento de riego dentro del intervalo de tiempo definido como jornada de riego.

La metodología que se ha desarrollado y probado en distintos casos de estudio permite que una misma toma realice más de una petición por jornada. En tal caso, debe indicarse el tiempo mínimo que se permite entre dos eventos de riego consecutivos de una misma toma.

\subsubsection{Consignas de control}

El gestor de la red de riego se encarga de definir las consignas de funcionamiento de los elementos de control. Se admite que toda la red se alimenta de desde una única fuente de suministro, desde donde se impulsa el agua directamente a la red mediante una estación de bombeo. Se asume también que la estación de bombeo funciona siguiendo una consigna de presión impuesta a la salida, gracias a un automatismo capaz de generar las órdenes de arranque, paro y velocidad de giro (si se trata de una BVV) más adecuadas de cada bomba para entregar el caudal demandado en cada momento a la presión impuesta. Dicha consigna de presión es susceptible de ser modificada, pero, acorde a la experiencia adquirida a través de los casos de estudio, los sistemas de control no permiten su modificación de una manera muy dinámica. Por lo tanto, para el algoritmo de optimización, las consignas de presión de las estaciones de bombeo serán impuestas como dato de partida, sin perjuicio de que se pueda realizar un análisis en profundidad evaluando los distintos resultados al variar dicha consigna mediante un proceso iterativo.

Además de las consignas de las estaciones de bombeo, las reglas de control del llenado de los depósitos intermedios, si los hubiere, también deben prefijarse de antemano. Aunque la metodología contempla esta casuística, siguiendo las conclusiones que se derivarán del presente capítulo, la recomendación es simplificar al máximo los sistemas a 
modelar siempre que sea posible, mediante su división en subsistemas independientes más pequeños.

\subsubsection{Metodología, variables del problema y su codificación}

Hasta ahora se han descrito las condiciones que el gestor ha de determinar de antemano y que el algoritmo debe respetar. El grado de libertad del que dispone el algoritmo de optimización es la elección de los instantes en que cada evento de riego debería efectuarse para conseguir que la estación de bombeo funcione en la medida de lo posible en los puntos de mayor rendimiento. Además, puede incorporarse, por un lado, el coste energético de la tarifa correspondiente, cuya diferenciación por periodos tiene una influencia decisiva en los resultados de la optimización y, por otro lado, las penalizaciones por exceso de potencia contratada.

La metodología de optimización propuesta se basa en este caso, dada la complejidad del problema a resolver, en aplicar un algoritmo genético (AG). Las variables de decisión se codifican en un cromosoma o vector de números enteros con tantos elementos como peticiones de riego se efectúen. Cada elemento o gen determina el instante en el que se debe iniciar el evento de riego correspondiente. Se asume que, una vez iniciado el riego, la toma permanece abierta hasta que finaliza el tiempo de riego indicado en la petición, o estimado a partir del volumen solicitado y el caudal de la toma.

La interpretación del valor que presenta cada gen depende de la discretización temporal que se haya efectuado. El instante de inicio del evento de riego coincidirá con el instante de inicio de la jornada de riego, más el valor del gen multiplicado por el intervalo de discretización. La Tabla 2 muestra un ejemplo de las relaciones mencionadas, tomando como intervalo de discretización una duración de 15 minutos.

Finalmente, el valor de cada gen debe estar acotado inferiormente por $0 \mathrm{y}$ superiormente por el número de intervalos en que se ha discretizado la jornada de riego menos el número de intervalos correspondientes al tiempo de riego que se haya peticionado. 
Tabla 2. Ejemplo de codificación de un cromosoma o vector de variables del problema de optimización.

\begin{tabular}{lllllll}
\hline Toma & Petición & $\begin{array}{l}\text { Tiempo } \\
\text { riego }\end{array}$ & Gen & $\begin{array}{l}\text { Inicio jornada } \\
\text { riego }\end{array}$ & $\begin{array}{l}\text { Inicio evento } \\
\text { riego }\end{array}$ & Fin evento riego \\
\hline T1 & T1a & $00: 30$ & 10 & $7 / 6 / 1922: 00$ & $8 / 6 / 190: 30$ & $08 / 06 / 191: 00$ \\
T1 & T1b & $01: 00$ & 19 & $7 / 6 / 1922: 00$ & $8 / 6 / 192: 45$ & $08 / 06 / 193: 45$ \\
T2 & T2a & $00: 45$ & 4 & $7 / 6 / 1922: 00$ & $7 / 6 / 1923: 00$ & $07 / 06 / 1923: 45$ \\
T3 & T3a & $1: 30$ & 25 & $7 / 6 / 1922: 00$ & $8 / 6 / 194: 15$ & $08 / 06 / 195: 45$ \\
T4 & T4a & $00: 15$ & 8 & $7 / 6 / 1922: 00$ & $8 / 6 / 190: 00$ & $08 / 06 / 190: 15$ \\
T4 & T4b & $00: 30$ & 11 & $7 / 6 / 1922: 00$ & $8 / 6 / 190: 45$ & $08 / 06 / 191: 15$ \\
T4 & T4c & $00: 15$ & 0 & $7 / 6 / 1922: 00$ & $7 / 6 / 1922: 00$ & $07 / 06 / 1922: 15$ \\
$\ldots$ & $\ldots$ & & $\ldots$ & $\ldots$ & $\ldots$ & $\ldots$ \\
\hline
\end{tabular}

\subsubsection{Función objetivo}

El valor de la función objetivo que indica la bondad de cada una de las soluciones se calcula como la energía total consumida a lo largo del periodo de cálculo completo, más un término que penaliza las soluciones que incurren en déficits de presión. Nótese que el valor que tome la función objetivo únicamente podrá expresarse en unidades de energía en las soluciones que no incurran en déficit de presión. El cálculo se detalla en la Ecuación (11).

$$
F O=\sum_{t=1}^{N t}\left(\frac{\gamma Q(t) H}{\eta(t)} \cdot \Delta t\right)+F_{p} \frac{1}{N_{h}} \cdot \sum_{h=1}^{N h} \max \left\{\left(P_{\text {min,req }}^{h}-P_{\text {min, calc }}^{h}\right), 0\right\}
$$

Donde:

- $\quad N t$ es el número de intervalos de tiempo en que se discretiza la jornada de riego.

- $\quad \gamma$ es el peso específico del agua $\left(\mathrm{N} / \mathrm{m}^{3}\right)$.

- $Q(t)$ es el caudal total bombeado en el instante $t\left(\mathrm{~m}^{3} / \mathrm{s}\right)$. Cabe mencionar que este caudal es función del cromosoma o vector de variables del problema.

- $\quad H$ es la altura entregada por las bombas. Se asume que es un valor constante derivado de la consigna impuesta en el controlador local (m).

- $\quad \eta(t)$ es el rendimiento global de la estación de bombeo en el instante $t$. Dicho rendimiento será función de las bombas en marcha, su velocidad de giro, el caudal de cada bomba y la altura de impulsión común. Sin embargo, fijada la altura y conocidas las reglas de operación establecidas por el control local de la 
E.B., finalmente puede fijarse el valor del rendimiento con una función exclusiva del caudal bombeado $\eta=\eta(Q)$

- $\Delta t$ es la duración del intervalo de discretización, a lo largo del cual se asume que el caudal es constante. Si queremos obtener el resultado en $k W h$, habrá que expresarlo en horas.

- $\quad F_{p}$ es el factor de penalización del déficit de presión. Cuanto mayor sea este factor, mayor es la probabilidad de expulsar del conjunto de soluciones aquellas que incurran en déficits de presión positivos.

- $\quad N_{h}$ es el número de tomas con al menos una petición de riego.

- $\quad P_{\text {min,req }}^{h}$ es la presión mínima requerida en la toma $h$. Este parámetro tiene una importancia fundamental en el contexto de la eficiencia energética. Cuanto menor sea este requerimiento, mayor será capacidad potencial de reducir la presión de consigna de la estación de bombeo y, por tanto, de reducir el consumo energético por unidad de volumen. No obstante, la presión mínima debe ajustarse a las prescripciones de las subunidades de riego instaladas en la parcela.

- $\quad P_{\text {min,calc }}^{h}$ se obtiene a partir de los resultados de la simulación en EPANET como la presión mínima calculada en la toma $h$ a lo largo de todos los instantes de tiempo que coincidan con un evento de riego (caudal de la toma positivo).

Teniendo en cuenta que el volumen total entregado a cada toma, expresado en $\mathrm{m}^{3}$, debe ser igual al volumen demandado por cada usuario $D_{h}$, el problema de optimización se puede expresar de la siguiente manera:

$$
\begin{gathered}
\min \{\mathrm{FO}\} \\
\text { s. a.: } \quad \sum_{t=1}^{N t} q_{h}(t) \Delta t=D_{h} \quad \forall h
\end{gathered}
$$

donde $q_{h}\left(\mathrm{~m}^{3} / \mathrm{h}\right)$ es el caudal entregado a cada toma $h$ en el instante $t$.

\subsubsection{Simulación hidráulica de los diferentes escenarios}

Cada uno de los escenarios se simula mediante el módulo de herramientas de EPANET (Rossman, 2000; Vegas Niño et al., 2017) con el fin de obtener los caudales y presiones en cada instante de simulación para cada toma de riego. Destacar que la elección del software de simulación no afectaría a la solución del problema. En todo caso, podría 
afectar al tiempo de cálculo, pero EPANET ha demostrado ser muy eficiente en este aspecto (Alvarruiz, Martínez-Alzamora, y Vidal 2015)

\subsubsection{Desdoblamiento del problema en periodo de bombeo y periodo de gravedad}

Entre las particularidades que se pueden encontrar en los sistemas de riego, hay una que cobra especial relevancia en el contexto de la optimización energética. La distribución topográfica de las tomas dentro de la red de distribución puede permitir que algunas de las tomas sean alimentadas exclusivamente con la energía disponible gracias a la diferencia de cota entre el punto de suministro y el punto de consumo, sin necesidad de contar con el aporte energético de la estación de bombeo.

Es estos casos, el problema de optimización se puede desdoblar en dos subproblemas, la maximización del volumen entregado por gravedad y la minimización del consumo energético de la estación de bombeo.

Como paso previo, debe subdividirse la jornada de riego en dos periodos diferenciados. El periodo de gravedad estará destinado a aportar el máximo volumen posible sin coste energético en esta etapa de distribución (es decir, a partir del depósito de cabecera, no se tiene en cuenta aquí el coste energético del transporte del agua hasta el depósito), lo que equivale a activar el mayor número de tomas siempre que se garanticen las presiones mínimas de servicio. Las peticiones de riego que no hayan podido incluirse en el periodo de gravedad, pasarán al conjunto de peticiones a satisfacer en el periodo por bombeo. El periodo de bombeo se plantea y resuelve tal como se ha descrito en el apartado anterior.

\subsubsection{Variables de decisión para maximizar el volumen entregado por gravedad}

Para la resolución de este subproblema se consideran como variables únicamente las peticiones de riego correspondientes a tomas cuya diferencia de cota con respecto a la lámina libre del depósito o balsa de cabecera es superior a la presión mínima requerida (m.c.a.) en la toma. Algunas de estas tomas podrán efectuar el riego por gravedad y otras puede que deban pasar al periodo de bombeo por no poder cumplir con la presión mínima requerida a causa de las pérdidas por fricción. 


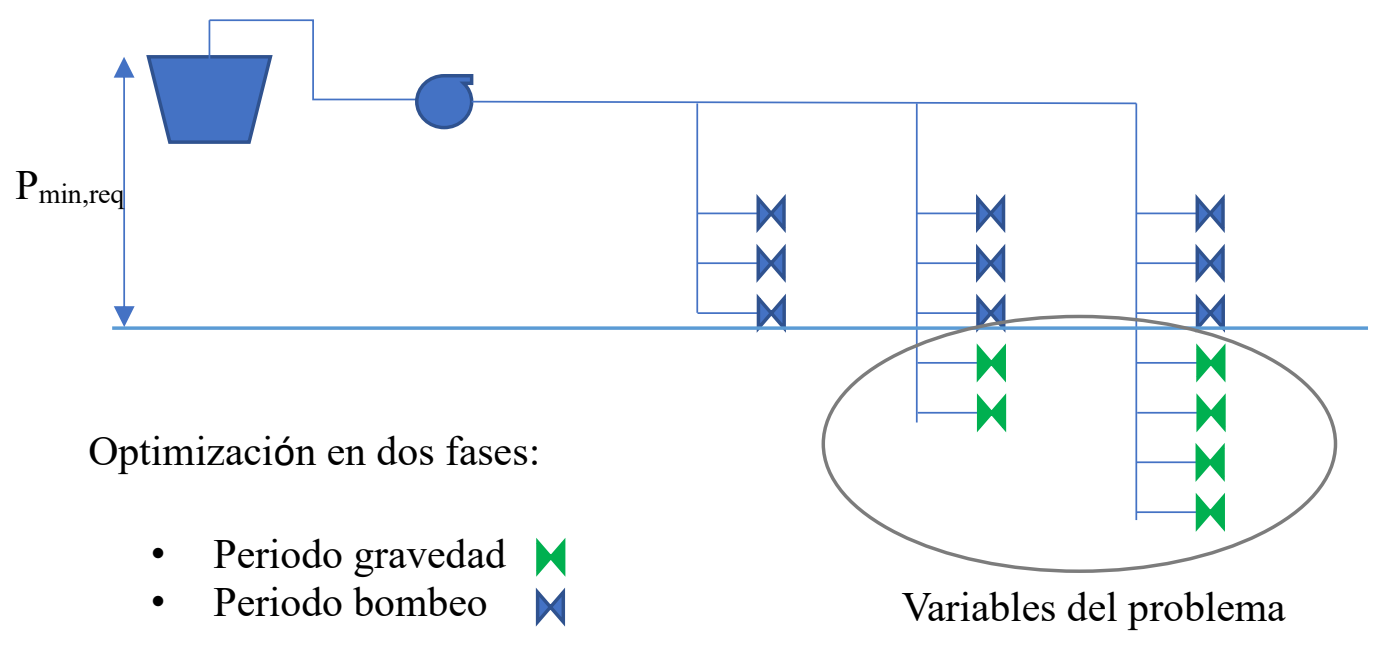

Figura 1. Esquema ilustrativo de la identificación de variables para el subproblema de maximizar el volumen entregado por gravedad.

La codificación de las variables es similar a la descrita para el problema general. Cada elemento del cromosoma configurado por las variables de decisión indica mediante un número entero el instante en que se inicia el evento de riego. Sin embargo, existe una diferencia importante. En este caso no existe la obligación de que todas las peticiones de riego sean satisfechas dentro del periodo de gravedad. Como se ha dicho, aquellas que no se puedan incluir pasarán a formar parte del conjunto de peticiones a satisfacer obligatoriamente en el periodo de bombeo.

Afinando más, las peticiones pueden incluso satisfacerse parcialmente durante el periodo de gravedad, finalizando durante el bombeo. Para lograr esto, basta con modificar la estrategia de codificación del cromosoma definida en el apartado 3.2.2.5 aumentando la cota superior permitida a las variables al número total de intervalos de tiempo en que se ha discretizado el periodo de gravedad más uno. De este modo, un evento de riego puede iniciarse y no finalizar, o directamente no iniciarse cuando el valor de la variable coincide con la cota superior.

\subsubsection{Función objetivo del problema de maximización del volumen entregado por gravedad}

La función objetivo se deriva directamente de la función definida para el caso general. Para conservar el sentido de la optimización como la minimización de una función, se ha modificado el signo del término que calcula el volumen total entregado. De esta forma, para escenarios en los que no haya déficit de presión, la función objetivo cambiada de signo tendrá unidades de $\mathrm{m}^{3}$. 
$F O=\sum_{t=1}^{N t}(-Q(t) \cdot \Delta t)+F_{p} \frac{1}{N_{h}} \cdot \sum_{h=1}^{N h} \max \left\{\left(P_{\text {min,req }}^{h}-P_{\text {min, calc }}^{h}\right), 0\right\}$

Finalmente, el problema de optimización puede formularse como sigue:

$$
\begin{gathered}
\min \{\mathrm{FO}\} \\
\text { s.a.: } \quad \sum_{t=1}^{N t} q_{h}(t) \Delta t \leq D_{h} \quad \forall h
\end{gathered}
$$

\subsubsection{Caso de estudio. Comunidad de regantes de "El Realón"}

Previamente a la realización de la presente Tesis, en el grupo de investigación se había trabajado en la optimización energética de la red de riego del Realón, mediante la implementación de la metodología descrita en el presente apartado y que se deriva de los trabajos realizados por los directores de esta Tesis. El objetivo principal de la ejecución de este estudio fue el de conocer y analizar a fondo las metodologías existentes para posteriormente tratar de realizar aportaciones que mejorasen sus capacidades.

En este apartado, pues, se describe el caso de estudio, así como los resultados obtenidos mediante la aplicación del método general de optimización energética de redes de riego.

Para definir el escenario de optimización, se utilizó como peticiones de riego los tiempos extraídos de la programación real que se ejecutó durante el verano del año 2013. Al disponer de la programación real, se pudieron comparar los resultados de la optimización con la simulación del escenario real.

\subsubsection{Descripción de la red de riego}

La comunidad de regantes "El Realón" de Picassent se encuentra situada en Valencia $\left(39^{\circ}, 23^{\prime}, 30^{\prime \prime} \mathrm{N}, 0^{\circ}, 28^{\prime}, 20^{\prime}\right.$ ' W). En la parcela 294 del polígono 34 del Término Municipal de Picassent se emplaza el cabezal de riego comunitario, el cual domina una superficie de riego de 251 hectáreas. 


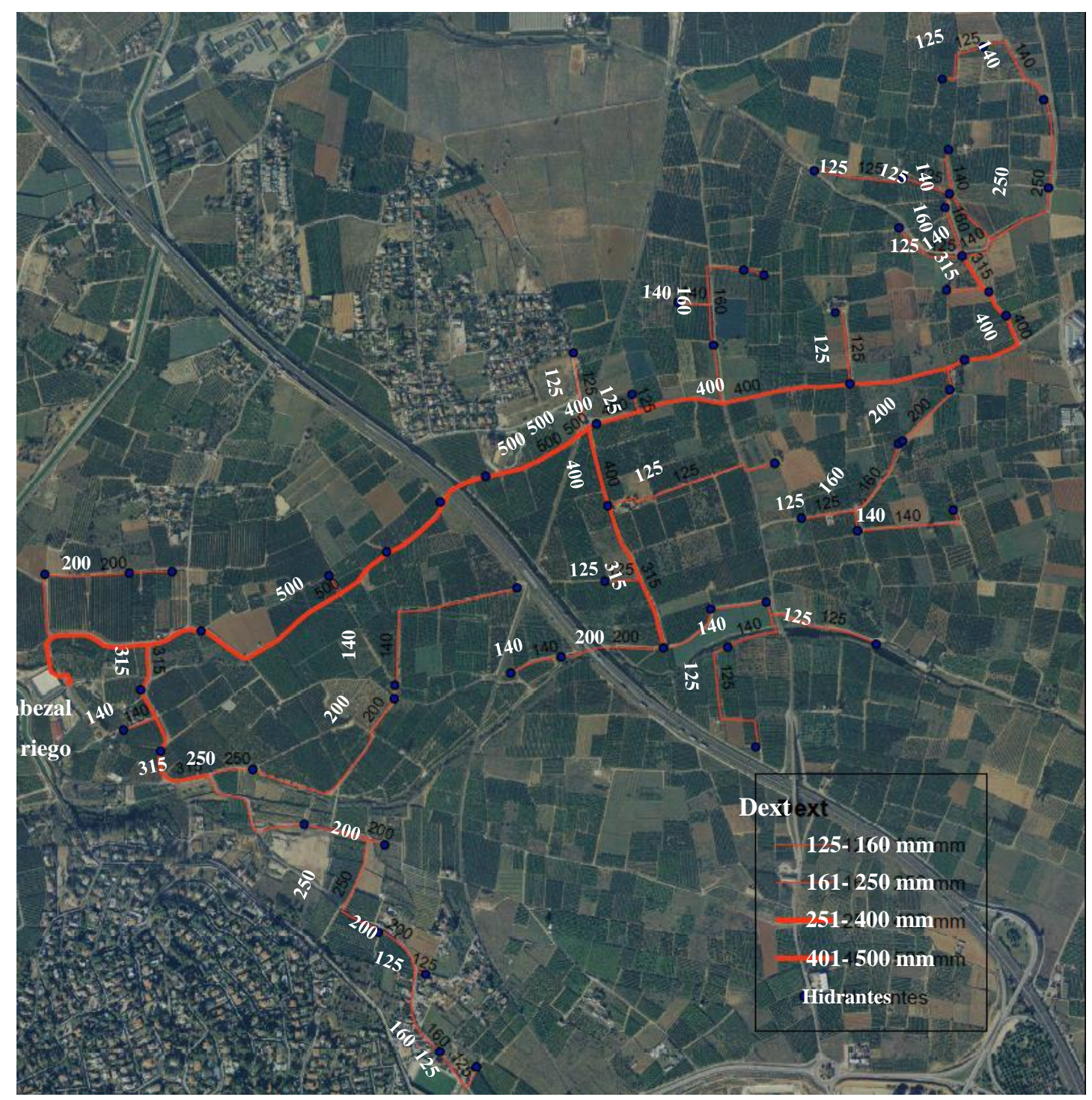

Figura 2. Vista general de la red de riego de Realón.

En esta misma parcela se ubica la balsa de regulación alimentada por gravedad desde el canal Júcar-Turia. La balsa se sitúa a una cota de 111,42 msnm, y la estación de bombeo está 3m por debajo de la altura máxima de la lámina libre de la balsa. La oscilación observada en funcionamiento del nivel de la lámina libre de la balsa es inferior a $1 \mathrm{~m}$.

La estación de bombeo consta de cuatro bombas idénticas modelo BEV-1226/4. Son bombas verticales con 4 rodetes centrífugos en serie y un motor de arrastre eléctrico de 45 kW de potencia nominal, girando a 1450 r.p.m. y alimentado con una tensión de $400 \mathrm{~V}$. El caudal y altura de diseño son $191 \mathrm{~m}^{3} / \mathrm{h}$ y 55,7 m.c.a. respectivamente. No obstante, las bombas funcionan con 3 de los 4 rodetes porque los técnicos encargados de la gestión de la CR observaron que la altura demandada era considerablemente menor que la altura que proporcionan las bombas en los puntos de funcionamiento cercanos al de rendimiento 
óptimo. De esta manera, simplemente eliminando uno de los cuatro rodetes de cada bomba se mejoró el rendimiento global de la estación de bombeo.

De las cuatro bombas disponibles, hay tres en estado activo y una está reservada para el caso de que exista una avería. De las tres bombas activas, una de ellas funciona a velocidad fija y las otras dos disponen de variador de velocidad. Periódicamente, una de las bombas activas pasa a ser la de reserva con el objetivo de equiparar el desgaste en las cuatro unidades.

La red de distribución está constituida por una red de tuberías de PVC (policloruro de vinilo) de diámetros comprendidos entre $125 \mathrm{~mm}$ y $500 \mathrm{~mm}$ con una longitud total de 14.726 metros que distribuyen el agua a 62 hidrantes multiusuario con un total de 362 tomas.

Actualmente cada jornada de riego se divide en dos periodos. Durante las horas comprendidas entre las 00:00 y las 10:00, cuando la electricidad es más barata, riegan aquellas tomas que necesitan energía extra de las bombas para poder cumplir con el requerimiento de presión en el hidrante (que ha de ser, como mínimo, de 25 m.c.a.), y entre las 10:00 y las 24:00, cuando la electricidad es más cara, riegan las tomas que tienen suficiente diferencia de cota entre la balsa y el hidrante como para asegurar la presión mínima en funcionamiento. Sin embargo, la distinción entre tomas a presión y tomas por gravedad no está optimizada.

\subsubsection{Modelado de la estación de bombeo}

\section{Curvas características}

La estación de bombeo consta de 3 bombas activas iguales cuyas curvas proporcionadas por el fabricante se muestran a continuación.

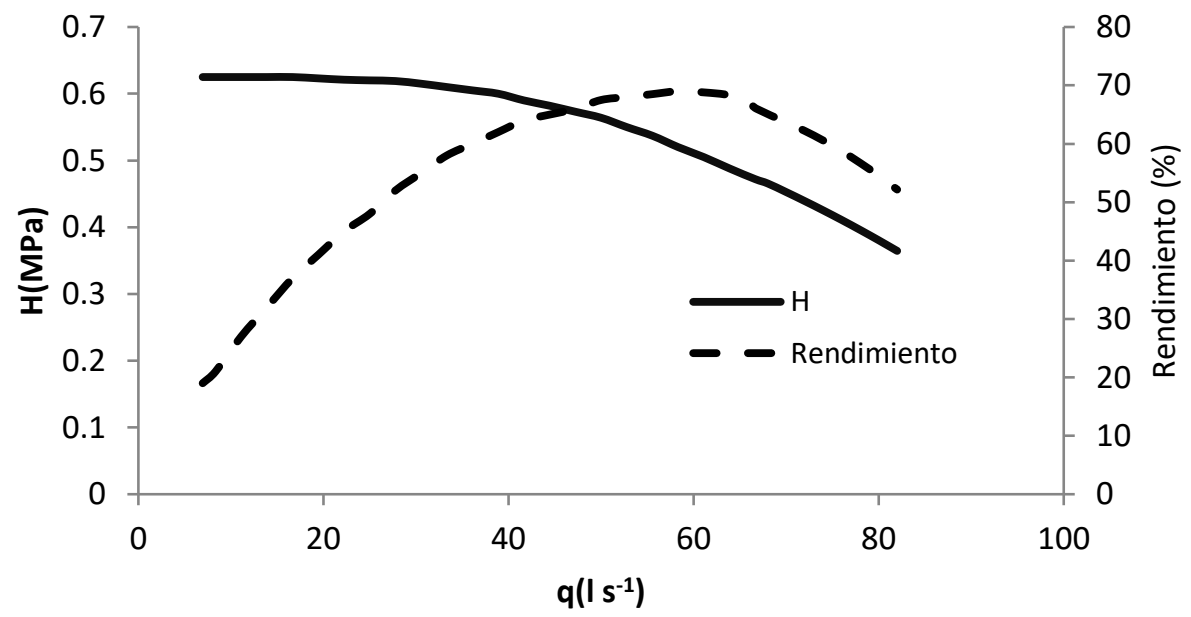

Figura 3. Curvas de presión y rendimiento en función del caudal de las bombas BEV-1226/4 
Tal como se comentó anteriormente, las bombas originalmente cuentan con 4 rodetes, pero actualmente trabajan únicamente con tres, puesto que la curva resultante se adapta mejor a las exigencias de la red. Se han calculado dichas curvas en base a los datos proporcionados por el fabricante y bajo el supuesto de que en el acoplamiento en serie de los rodetes circula el mismo caudal por cada uno de ellos y se eleva la altura en la misma proporción en cada etapa. Por tanto, cada nuevo punto de la curva de altura se obtendrá multiplicando el original por el factor 0,75 . También se supone que la eliminación de un rodete no afecta al rendimiento global, obteniendo como resultado las curvas representadas en la Figura 4.

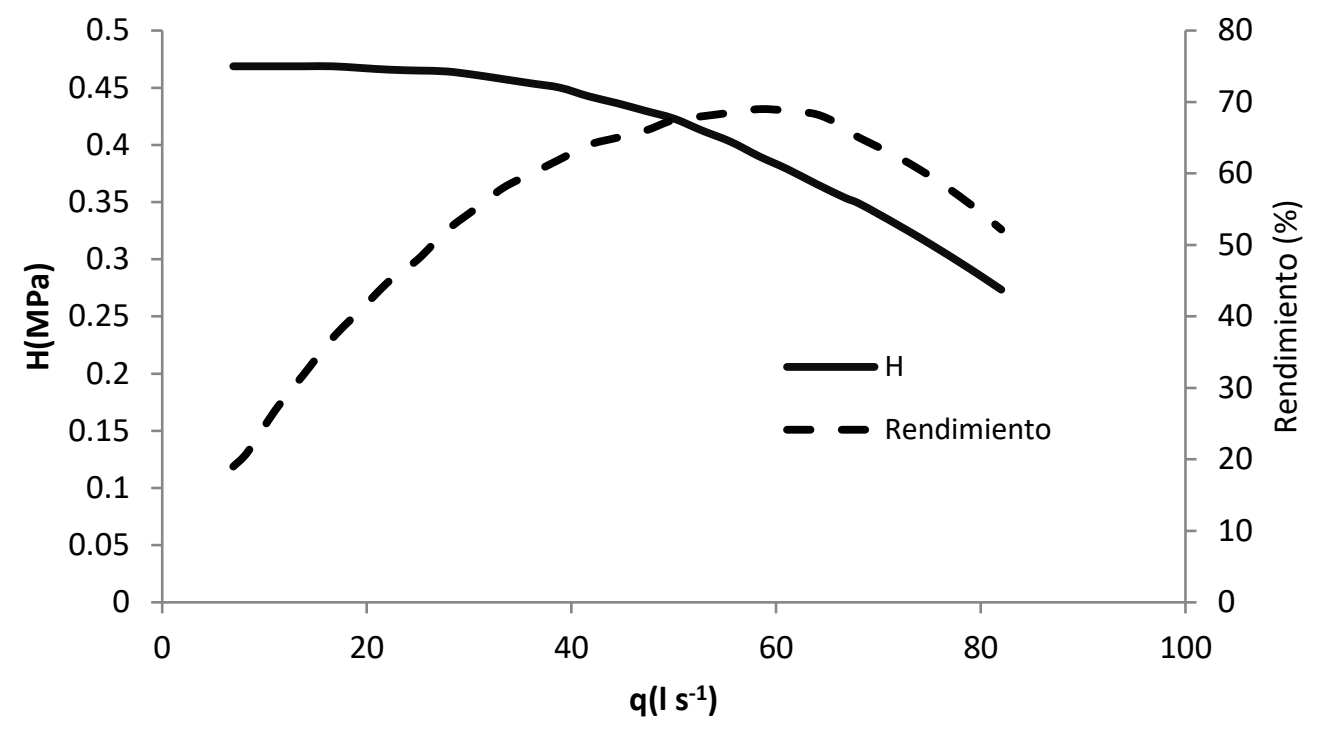

Figura 4. Curvas de presión y rendimiento en función del caudal para las bombas con 3 rodetes.

A continuación, se ajustan los puntos de las dos curvas en la zona de trabajo a una parábola mediante mínimos cuadrados, obteniendo las siguientes expresiones:

$$
\begin{gathered}
H=45,639+0,1609 Q-0,00468 Q^{2} \\
\eta=0,02506 Q-0,000228 Q^{2}
\end{gathered}
$$

\section{Secuencia de arranque}

La estación de bombeo cuenta con una bomba de velocidad fija y dos con variador de velocidad. El variador de velocidad cuenta con un controlador automático al cual se le introduce como parámetro de consigna la presión requerida en cabecera. La ventaja que presenta el hecho de disponer de bombas de velocidad variable (al menos una) en la estación de bombeo, es que para cada demanda de caudal (hasta el límite en que las tres bombas 
funcionan al 100\% de la velocidad) se puede entregar justo la presión de consigna deseada. Por el contrario, si todas las bombas fueran de velocidad fija, la presión a la salida de la bomba variaría en función del caudal demandado, siendo generalmente distinta a la de consigna.

A caudales bajos se activa únicamente una de las bombas con variador de velocidad. Esta va aumentando su velocidad a medida que aumenta la demanda, hasta que llega a su velocidad nominal. En ese momento se conecta la segunda bomba de velocidad variable, girando ambas a la misma velocidad. A partir del punto en que la demanda de caudal es mayor del que pueden entregar las dos bombas a la presión de consigna y funcionando al $100 \%$ de la velocidad nominal, pasa a activarse la bomba de velocidad fija.

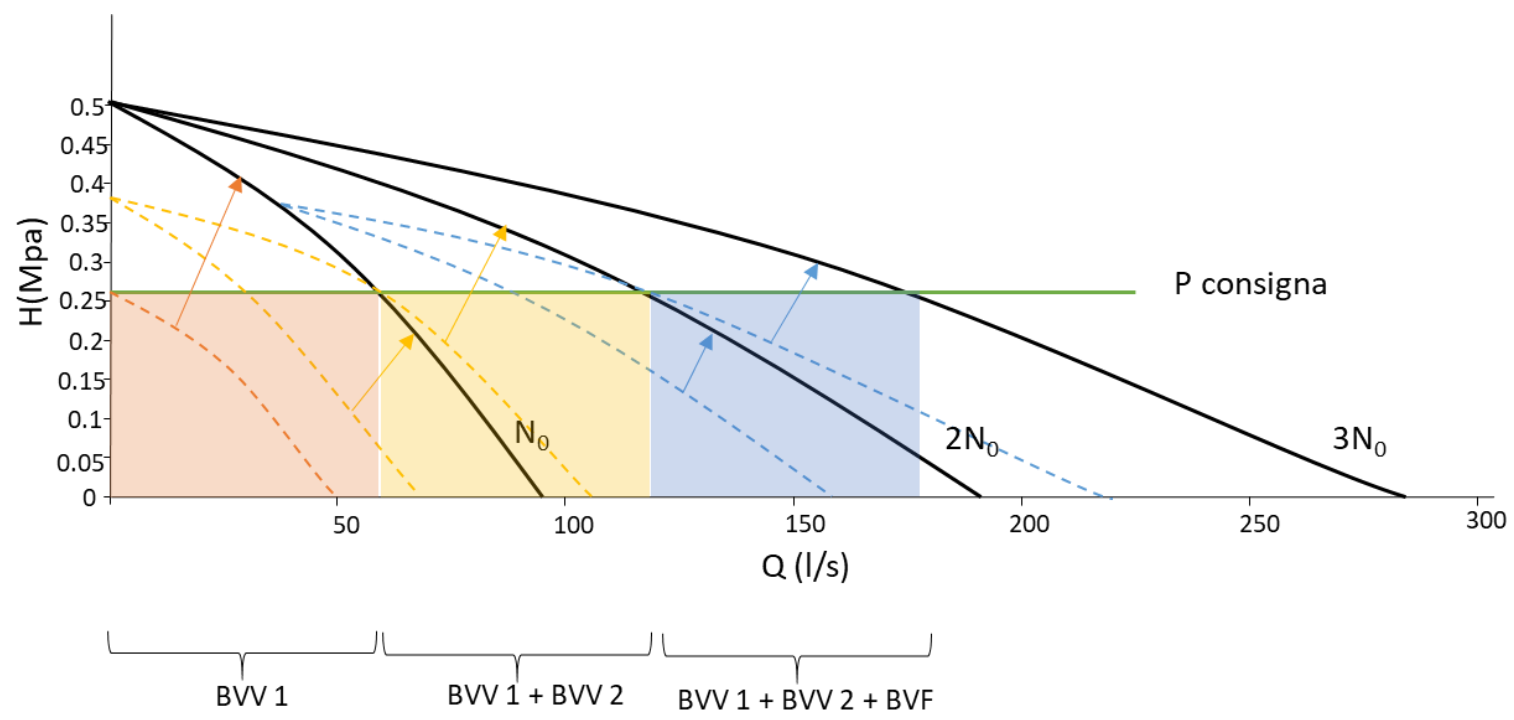

Figura 5. Curvas de altura-caudal de las bombas para la regulación de la estación de bombeo del cabezal de Realón.

\section{Cálculo de parámetros}

El parámetro principal a calcular, requerido por el Algoritmo Genético para evaluar la función objetivo, es el rendimiento global de la estación de bombeo para cada caudal demandado. En cada instante de simulación se generan unas necesidades de caudal $\left(\mathrm{Q}_{\mathrm{r}}\right)$ y se fija una presión en cabecera $\left(\mathrm{H}_{\mathrm{r}}\right)$. A partir de estos dos parámetros se calcula el punto de funcionamiento, es decir, qué bombas están activas, a qué velocidad funcionan las bombas con variador y qué caudal trasiega cada una.

Como los escenarios de consumo se generan aleatoriamente, en muchos de los casos el caudal o la altura que se introducen en la función están fuera de los límites de actuación de la estación de bombeo. Para penalizar tales casos no factibles, la función devuelve un 
valor de rendimiento cercano a cero, de modo que el valor de la energía calculada resulte muy elevado, y con ello también la función objetivo, descartando el escenario (o cromosoma) para la siguiente generación.

Para los casos factibles el proceso de cálculo es el siguiente. En primer lugar, se calcula el caudal ( $\left.Q_{\text {corte }}\right)$ que trasegaría una de las bombas para la presión de consigna $\left(\mathrm{H}_{\mathrm{r}}\right)$ y velocidad nominal, mediante la curva H-Q común para las tres bombas, ajustada a una parábola. A continuación, se distinguen tres casos:

a) El caudal requerido $Q_{r}$ es mayor que dos veces el $Q_{\text {corte. }}$

En este caso las tres bombas estarían activas. El caudal que circula por la bomba de velocidad fija $\left(\mathrm{Q}_{1}\right)$ coincide con el caudal calculado en el paso previo $\mathrm{Q}_{\text {corte. }}$ El caudal restante se ha de repartir igualmente entre las dos bombas de velocidad variable, ya que se trata de dos bombas idénticas trabajando al mismo régimen de giro. Por tanto, por cada una de ellas circulará el caudal:

$$
Q_{2}=Q_{3}=\frac{Q_{r}-Q_{\text {corte }}}{2}
$$

Para calcular el rendimiento de la bomba de velocidad fija se usa directamente la Ecuación (16), obteniendo $\eta_{1}$ en función de $Q_{1}$. Para calcular el rendimiento de las bombas de velocidad variable se aplican las leyes de semejanza. El primer paso es obtener el punto homólogo al de funcionamiento en la curva correspondiente a la velocidad nominal. Para ello se ha de resolver el siguiente sistema de ecuaciones:

$$
\left\{\begin{array}{c}
H_{h o m}=45,639+0,1609 Q_{h o m}-0,00468 Q_{h o m}^{2} \\
H_{h o m}=\frac{H_{r}}{Q_{2}^{2}} Q_{h o m}^{2}
\end{array}\right.
$$

donde $H_{r}$ y $Q_{2}$ son conocidos (altura y caudal requeridos en cabecera por cada BVV). Una vez calculado el caudal en el punto homólogo, se obtiene el rendimiento $\eta_{1}=\eta_{2}$ de las dos bombas con variador de velocidad para dicho punto directamente mediante la Ecuación (16).

A continuación, se calcula el factor de reducción de la velocidad a partir de la relación entre el caudal en el punto de funcionamiento y el caudal en el punto homólogo:

$$
\alpha=\frac{Q_{2}}{Q_{\text {hom }}}
$$


Por último, se calcula el rendimiento global conjunto de las tres bombas, teniendo en cuenta que la potencia total consumida es la suma de potencias individuales.

$$
\begin{gathered}
P=P_{1}+P_{2}+P_{3} \\
\frac{\gamma Q_{r} H_{r}}{\eta}=\frac{\gamma Q_{1} H_{r}}{\eta_{1}}+\frac{\gamma Q_{2} H_{r}}{\eta_{2}}+\frac{\gamma Q_{3} H_{r}}{\eta_{3}} \\
\eta=\frac{Q_{r}}{\frac{Q_{1}}{\eta_{1}}+\frac{Q_{2}}{\eta_{2}}+\frac{Q_{3}}{\eta_{3}}}
\end{gathered}
$$

El caudal requerido $Q_{r}$ es menor que el $Q_{\text {corte. }}$

En este caso únicamente se activa una de las dos bombas de velocidad variable. El caudal que trasiega, por tanto, coincide con el caudal requerido. El rendimiento de la bomba coincide con el rendimiento global y se calcula tal como se ha explicado en el apartado anterior, mediante las ecuaciones (18) y (16). También se puede calcular el factor $\alpha$ mediante la Ecuación (19).

El caudal requerido es mayor que $Q_{\text {corte }} y$ menor que $2 \cdot Q_{\text {corte }} \cdot$

Las dos bombas de velocidad variable estarían activas. El caudal que trasegaría cada una de ellas es:

$$
Q_{2}=Q_{3}=\frac{Q}{2}
$$

El rendimiento global en este caso coincide con el rendimiento de cada una de las bombas, puesto que ambas trabajan en el mismo punto de funcionamiento, tienen el mismo rendimiento y trasiegan el mismo caudal.

\subsubsection{Tomas regadas por gravedad}

La red de riego de Realón presenta la singularidad de que ciertas tomas están situadas a una cota suficientemente inferior a la cota de la balsa de riego como para garantizar la presión mínima en el hidrante cuando la toma está abierta.

Para el caso de riego analizado la programación del riego por gravedad tiene una duración de 14 horas. Las 10 horas restantes hasta completar la jornada de riego se emplearán 
para satisfacer, con la participación del bombeo, las peticiones que no hayan entrado en el periodo de gravedad.

La presión mínima requerida en cada hidrante operativo se ha fijado en 25 m.c.a. El tiempo de riego de cada toma está definido por el propio usuario. El caudal que entrega cada toma abierta se ha considerado constante y se ha calculado en base a datos recompilados de consumo real.

El periodo de gravedad se ha discretizado en intervalos de cálculo de 5 minutos, por lo que el problema completo constará de 168 intervalos, correspondientes a las 14 horas de riego asignadas. El objetivo es este primer paso es entregar el máximo volumen posible durante este periodo.

\subsubsection{Tomas que necesitan energía adicional}

El siguiente paso en este proceso tiene como objetivo realizar el suministro a las peticiones restantes durante un tiempo total de 10 horas y con el menor coste energético posible.

Las variables que se consideran para resolver el problema son justamente aquellas tomas que no han realizado el riego durante el periodo sin bombeo, tanto aquellas con una diferencia de cota respecto de la balsa menor de 25 metros, como las que no han finalizado el riego aun teniendo suficiente diferencia de cota.

La presión de consigna a la salida de la estación de bombeo se ha fijado ahora en un valor de 23 m.c.a. A partir de esta presión de consigna, los caudales propuestos en cada instante y el rendimiento de la estación de bombeo, calculado a partir del punto de funcionamiento, se obtiene la potencia eléctrica consumida en cada instante. Integrando a lo largo del periodo de cálculo dicha potencia se obtiene la energía total consumida en la jornada de riego.

\subsubsection{Resultados}

\section{Gravedad}

El tiempo de cálculo para alcanzar la convergencia, para el problema de maximizar el volumen entregado por gravedad, es de aproximadamente una hora. No obstante, el resultado que se presenta en este apartado se ha obtenido dejando el algoritmo calcular 
durante 3 horas aproximadamente. De este modo se ha conseguido aumentar el caudal total entregado alrededor de un $3 \%$, respecto del primer resultado.

La solución ofrecida verifica que la presión en las tomas activas es mayor que 25 m.c.a. en todos los instantes de cálculo.

El resultado global del volumen entregado en este periodo es de $2.370,5 \mathrm{~m}^{3}$. Este resultado supone un aumento importante respecto del volumen entregado inicialmente, que era de $1.635,08 \mathrm{~m}^{3}$, por lo que se ha mejorado un $45 \%$. Esta mejora tiene una influencia decisiva en el consumo energético total de la red de riego, puesto que se trata de un volumen entregado sin coste en esta etapa de distribución.

El número de tomas que efectivamente han finalizado el riego es de 166, quedando además 22 tomas que han iniciado, pero no finalizado su tiempo de riego. Se partía de un total de 193 tomas operativas.

Cabe comentar, que esta metodología tiende a establecer un caudal más o menos constante a lo largo de todo el periodo de cálculo. En la Figura 6 se presenta el perfil de caudal total resultante a lo largo del tiempo.

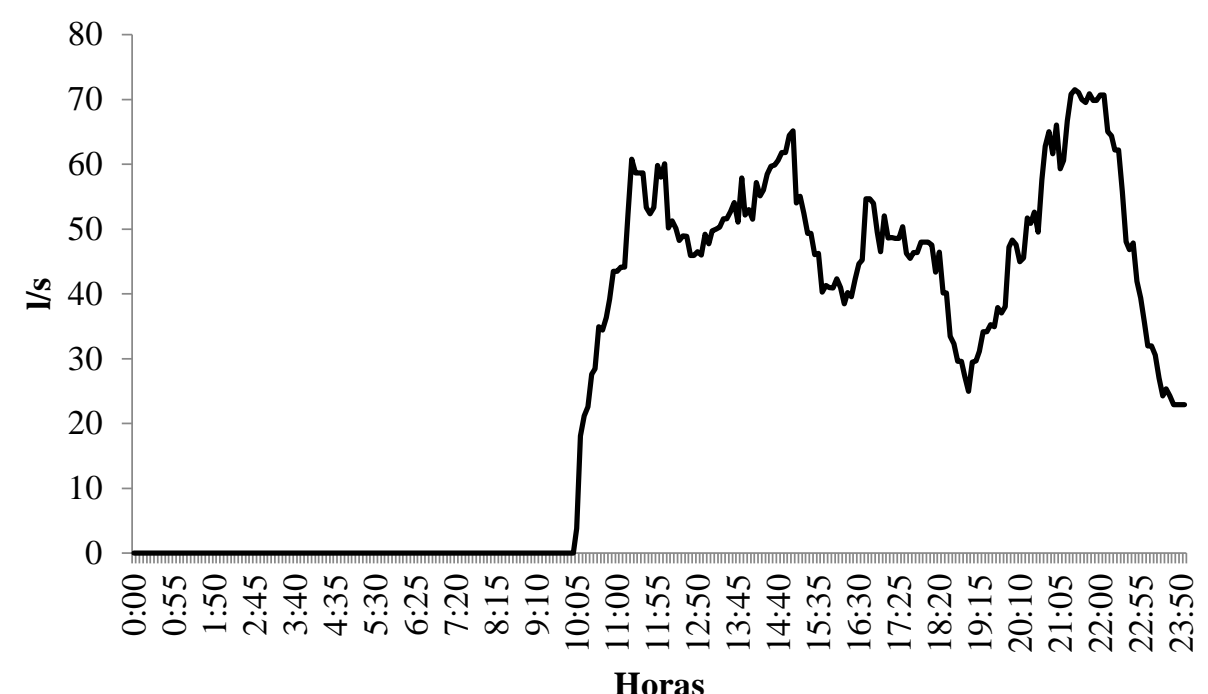

Figura 6. Caudal total entregado por gravedad a la red en función del tiempo. Metodología 1.

\section{Bombeo}

La reducción en el volumen a entregar durante el periodo de bombeo, junto con una mejor organización de los eventos de riego, permite reducir la presión de consigna de la 
estación de bombeo respecto a la consigna real sin perjudicar a las presiones de servicio. En concreto, se ha podido reducir la consigna desde los $28 \mathrm{~m}$ hasta $23 \mathrm{~m}$.

El algoritmo implementado para la optimización energética de la estación de bombeo converge igualmente en un tiempo de 1 hora aproximadamente.

La solución verifica que efectivamente no se producen errores (déficit de presión) en ningún momento. Con esto ya se tiene una mejora respecto de la programación inicial, en la que se observaron defectos de presión, especialmente durante las horas de riego por gravedad.

El valor objetivo corresponde con el mínimo de energía consumida en una jornada de riego, siendo su valor 432,59 kWh/día. Si se compara con el valor de energía consumida con la programación inicial, que era de 643,9kWh/día, para el mismo volumen entrega al cabo del día, se obtendría una reducción en el consumo energético de un 32,82\%.

En cuanto a la potencia máxima demandada por la estación de bombeo, se ha obtenido un resultado de $56,72 \mathrm{~kW}$. Esto supone una mejora considerable respecto de la programación inicial, en la que se alcanzaba una potencia máxima demandada de 84,78 kW. Esta reducción del 33\% repercutirá directamente en la posibilidad de reducir en la misma proporción la potencia contratada, con el consecuente ahorro económico, teniendo en cuenta además la subida de un $131 \%$ del término de potencia en la tarifa eléctrica de 2014.

En la Figura 7 se muestra el perfil de demanda de potencia a lo largo de una jornada de riego.

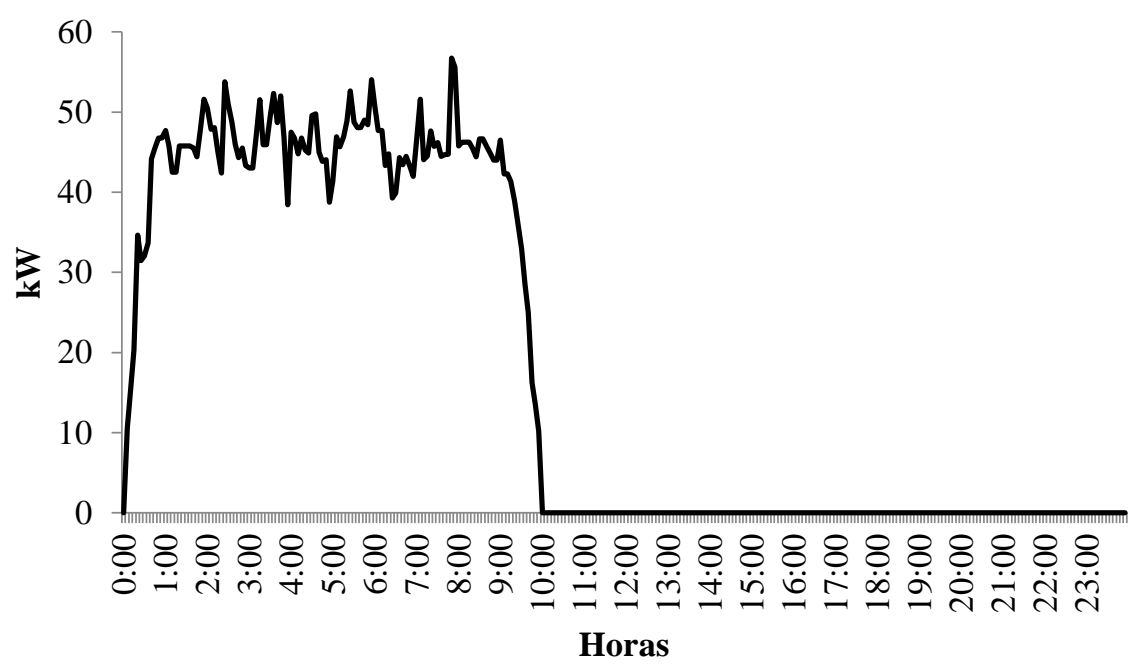

Figura 7. Demanda de potencia en función del tiempo. 
En la Figura 8 se muestra la evolución del caudal total entregado en función del tiempo para la jornada de riego.

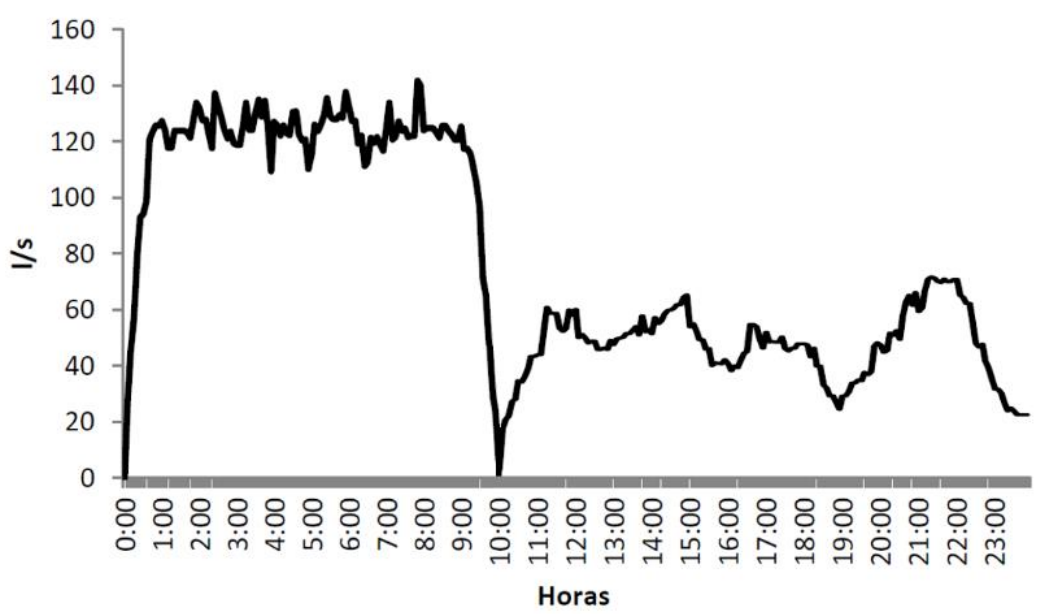

Figura 8. Caudal entregado en función del tiempo

En la Tabla 3 se comparan los resultados obtenidos mediante las metodologías descritas, con los valores calculados para la programación de riego inicial.

Tabla 3. Resumen valores principales antes y después de la optimización.

\section{Escenario inicial $\quad$ Escenario optimización}

$\begin{array}{lll}\text { V total }\left(\mathrm{m}^{3} / \text { día }\right) & 6.678,78 & 6.704 \\ \text { V gravedad (m³/día) } & 1.635,08 & 2.370,5 \\ \text { P consigna (m.c.a.) } & 28 & 23 \\ \text { Energía (kWh/día) } & 643,896 & 432,59 \\ \text { Potencia máx. }(\mathrm{kW}) & 84,78 & 56,72\end{array}$

\subsubsection{Conclusiones derivadas de aplicar la metodología descrita}

El método de optimización descrito ha demostrado ser de utilidad para conseguir un mejor reparto de las demandas de riego a lo largo de la jornada bajo criterios de eficiencia energética. En el caso de estudio se ha conseguido una reducción teórica del consumo energético del orden del $30 \%$ con respecto al escenario de operación real. La implementación de la metodología descrita conforma, pues, una herramienta útil que puede servir como sistema de soporte a la decisión para los gestores de las comunidades de regantes, cuyas 
redes estén alimentadas por inyección directa con estaciones de bombeo provistas de bombas de velocidad variable, y un controlador capaz de seguir una consigna de presión en la impulsión. Si, además, algunas tomas pueden regarse por gravedad, se ha demostrado que los ahorros pueden ser más significativos.

Sin embargo, tanto en el caso de estudio descrito en este capítulo como en los estudios previos en los que se basa la metodología propuesta, esta se ha utilizado hasta ahora solo como una herramienta estratégica para efectuar el reparto de los riegos de una forma puntual, calculando nuevas programaciones con periodicidad estacional o menor. Este hecho resulta entendible a la vista de los tiempos de computación necesarios hasta que el algoritmo converge a una solución, siendo del orden de algunas horas, para una red de riego no excesivamente grande.

Así, esta forma de proceder no resulta óptima puesto que las necesidades de agua de las distintas parcelas y, probablemente, distintos cultivos pueden variar sustancialmente durante el periodo de tiempo que discurre hasta que se actualizan las programaciones. La realidad es que día a día deben tomarse decisiones a nivel táctico para adaptar el riego a las circunstancias particulares que pueden ocurrir. Estas decisiones suponen cambios en las condiciones simuladas, y estos cambios desembocan en resultados distintos de los óptimos previstos.

Para poder tomar decisiones tácticas a corto plazo debe disponerse de un sistema de soporte a las decisiones capaz de ofrecer los resultados en un tiempo lo suficientemente reducido para permitir su operatividad y que pueda ser explotado por parte del personal gestor con una frecuencia diaria o menor.

En consecuencia, trasladar el método de optimización energética al ámbito del tiempo real requiere la realización de nuevas indagaciones que proporcionen, o bien una aceleración del algoritmo mediante modificaciones en el mismo, o bien un cambio de paradigma en cuanto a la metodología de optimización a utilizar.

\subsection{Mejoras en el método de optimización para acelerar los cálculos}

En el presente apartado se describen y validan dos mejoras al método general de optimización energética expuesto en el apartado anterior. Dicho método resolvía el problema de optimización de la programación de riego mediante un único objetivo. El método se 
basaba en un algoritmo genético, con ejecución computacional en un único hilo, en el que la función objetivo trata de minimizar el consumo total de energía, a la vez que se penaliza el déficit de presión en las tomas activas, a través de la programación más adecuada de cada una de las peticiones de riego.

El problema que se plantea ahora es el mismo, pero se ha desdoblado la función objetivo en dos: una función que minimiza el consumo energético y otra que minimiza el déficit de presión en las tomas activas. Así pues, el problema se abordada ahora desde una perspectiva multiobjetivo con una mejora añadida, que consiste en la ejecución del algoritmo trabajando con varios procesos en paralelo (programación multihilo). Como se verá más adelante, estas mejoras han conseguido reducir sustancialmente el tiempo de convergencia del algoritmo.

Gracias a estas novedades, se pudo implementar un optimizador de la programación diaria de riego y se aplicó a un proyecto piloto en la comunidad de regantes del Pantano Estrecho de Peñarroya (España).

En la bibliografía se han reportado varias técnicas para acelerar los algoritmos evolutivos. Existen técnicas basadas en la optimización de las funciones de mutación y cruce (Nia y Alipouri 2009), otras basadas en el uso de metamodelos (Rasheed, Ni, y Vattam 2005), otras basadas en potenciar la diversidad de la población (Jassadapakorn y Chongstitvatana 2011), y las hay también que se basan en aprovechar la computación multihilo (Sinha et al., 2015). La paralelización también ha sido aplicada con éxito en otros campos de investigación (Liu et al., 2018). En este trabajo, con el fin de reducir los tiempos de cálculo, se ha explotado una combinación entre la mejora de la diversidad poblacional y el cálculo multihilo, ya que las funciones de mutación y cruce ya fueron optimizadas en trabajos anteriores, mientras que el uso de metamodelos reduce la precisión de las simulaciones.

\subsubsection{Descripción del algoritmo}

Tal como se ha comentado anteriormente, los algoritmos metaheurísticos se adaptan muy bien para resolver problemas de tipo aquí planteado. De entre el conjunto de algoritmos metaheurísticos existentes, en la nueva propuesta se va a utilizar el algoritmo genético multiobjetivo conocido como NSGA-II (Non-dominated Sorting Genetic Algorithm), elección justificada principalmente por su convergencia más rápida en comparación con 
otros algoritmos del mismo tipo (Deb et al., 2002). Además, el NSGA-II se adapta fácilmente a problemas multiobjetivo. La elección de otros algoritmos podría afectar al tiempo de procesamiento, pero el objetivo de este estudio no era comparar algoritmos metaheurísticos entre sí, sino comparar la optimización paralela y multiobjetivo frente a la optimización mono-objetivo ejecutada en un único hilo. La implementación del algoritmo base se ha tomado del paquete JMetal (Durillo y Nebro 2011), disponible bajo licencia pública general GNU (del acrónimo recursivo "GNU is Not Unix").

La codificación del cromosoma se ha definido, de forma idéntica al método general, como una serie de números enteros. Cada gen corresponde a una petición de riego e indica el instante en que se ha de iniciar el mismo. La discretización temporal es otro factor clave en la complejidad del problema y, por tanto, en la eficiencia computacional. Se ha tratado de que esta sea lo más amplia posible, siempre que pueda enmarcar las distintas duraciones de las peticiones de riego. Para ello, se ha decidido elegir como intervalo de discretización el máximo común divisor del conjunto de duraciones, siempre que este sea mayor a cinco minutos. Este método permite sistematizar el problema con un planteamiento más general, que engloba el caso particular de la sectorización por turnos de la red, el cual vendría representado por un conjunto de peticiones de igual tiempo de riego.

En cuanto a la evaluación de la población, se ha propuesto un enfoque multiobjetivo en el que se trata de minimizar el coste total del bombeo, por un lado, y minimizar el déficit de presión de servicio, por otro. Hasta la fecha, el déficit de presión se manejaba como una penalización del coste en un enfoque mono-objetivo. Con el enfoque multiobjetivo, el hecho de no descartar soluciones a priori (que serían rechazadas en un algoritmo mono-objetivo) favorece la diversidad en la población. La mejora se basa en la hipótesis de que una mayor diversidad mejorará la tasa de convergencia y evitará el estancamiento en óptimos locales.

En este estudio, la función objetivo principal se ha modificado ligeramente para evaluar, no el consumo energético total, sino el coste económico en el que incurre dicho consumo de energía, incluyendo las posibles penalizaciones por exceso de energía. Este enfoque presenta una mayor utilidad para las comunidades de regantes, dado el potencial impacto que puede tener un ajuste de la potencia contratada en el gasto energético anual.

El coste total $C T$ de la jornada de riego se ha calculado considerando el rendimiento global de la estación de bombeo, la discriminación horaria de los precios de la energía y las 
penalizaciones por exceso de potencia considerando una tarifa de seis periodos, según la Ecuación (24). El coste asociado directamente a la potencia contratada no se ha contemplado por tratarse de un coste fijo.

$$
C T=\sum_{t=1}^{N t}\left(\frac{\gamma Q(t) H}{\eta(t)} \cdot \Delta t \cdot C e(t)\right)+\sum_{i=1}^{i=6} K_{i} \cdot 1,4064 \cdot \sqrt{\sum_{j=1}^{j=n}\left(P d_{j}-P c_{i}\right)^{2}}
$$

donde:

- $\quad N t$ es el número de intervalos de tiempo en que se discretiza la jornada de riego.

- $\gamma$ es el peso específico del agua $\left(\mathrm{N} / \mathrm{m}^{3}\right)$.

- $Q(t)$ es el caudal total bombeado en el instante $t\left(\mathrm{~m}^{3} / \mathrm{s}\right)$. Cabe mencionar que este caudal es función del cromosoma o vector de variables del problema.

- $\quad H$ es la altura entregada por las bombas. Se asume que es un valor constante derivado de la consigna impuesta en el controlador local (m).

- $\quad \eta(t)$ es el rendimiento global de la estación de bombeo en el instante t, el cual puede calcularse en función del caudal $Q(t)$ bombeado.

- $\Delta t$ es la duración del intervalo de discretización, a lo largo del cual se asume que el caudal es constante, expresada en horas.

- $C e(t)$ es el coste de la energía en función de la época del año y la hora del día $(€ / \mathrm{Wh})$.

- $K_{i}$ es un factor que depende del periodo tarifario y que toma los valores de la Tabla 4.

- $P c_{i}$ es la potencia contratada en el periodo $i(\mathrm{~kW})$.

- $P d_{j}$ es la potencia máxima demandada en cada cuarto horario $j$, dentro del periodo $i$, en que se ha superado $P c_{i}$. Hay que tener en cuenta que $j$ y $n$ dependen de la distribución de los periodos tarifarios a lo largo del día.

Tabla 4. Coeficiente para el cálculo de la penalización por exceso de potencia en tarifas de seis periodos. Publicado en BOE núm. 268, de 8 de noviembre de 2001, páginas 40618 a 40629 (https://www.boe.es/eli/es/rd/2001/10/26/1164)

\begin{tabular}{lllllll}
\hline Periodo & 1 & 2 & 3 & 4 & 5 & 6 \\
\hline$K_{i}(€ / \mathrm{kW})$ & 1 & 0.5 & 0.37 & 0.37 & 0.37 & 0.17 \\
\hline
\end{tabular}


Para el cálculo del segundo objetivo DMP (Déficit Medio de Presión) se ha observado de manera individualizada la presión mínima a satisfacer en cada uno de los hidrantes. Este parámetro tiene en cuenta la diferencia de cota entre la estación de bombeo y el hidrante, así como las pérdidas de carga en el trayecto desde la fuente hasta él. En cada solución se calcula, mediante la simulación con EPANET, la presión más desfavorable en los hidrantes activos y se compara con la consigna mínima. El valor objetivo para cada solución es el promedio de las diferencias entre la presión de consigna y la mínima calculada, siempre que dicha diferencia sea positiva, es decir, siempre que haya déficit de presión.

$$
D M P=\frac{1}{N_{h}} \cdot \sum_{h=1}^{N h} \max \left\{\left(P_{\text {min,req }}^{h}-P_{\text {min,calc }}^{h}\right), 0\right\}
$$

donde:

- $\quad N_{h}$ es el número de tomas con al menos una petición de riego.

- $\quad P_{m i n, r e q}^{h}$ es la presión mínima requerida en la toma $h$.

- $\quad P_{m i n, c a l c}^{h}$ se obtiene a partir de los resultados de la simulación en EPANET, como la presión mínima calculada en la toma $h$ a lo largo de los instantes de tiempo que coincidan con un evento de riego (caudal de la toma positivo).

Si $D_{h}\left(\mathrm{~m}^{3}\right)$ indica el volumen total demandado por una toma y $q_{h}(t)\left(\mathrm{m}^{3} / \mathrm{h}\right)$, el caudal saliente por la toma en los instantes en que está activa, el problema de optimización puede expresarse como sigue:

$$
\begin{gathered}
\min \{C T, D M P\} \\
\text { s.a.: } \quad \sum_{h=1}^{N h} q_{h}(t) \Delta t=D_{h}
\end{gathered}
$$

En todos los casos analizados se han mantenido constantes los parámetros característicos del Algoritmo Genético. El tamaño de la población se ha fijado en 500 cromosomas, el factor de cruzamiento en 0.9 y el de mutación en 1/número de variables.

Para comprobar la eficacia de los nuevos planteamientos se han realizado una serie de análisis cuya finalidad es demostrar que el enfoque multiobjetivo alcanza mejores resultados que el mono-objetivo con el mismo número de evaluaciones, y que el hecho de paralelizar el algoritmo utilizando procesadores multi-core, hoy en día presentes en cualquier ordenador, reduce efectivamente el tiempo de computación. El programa se ha ejecutado en 
una máquina con 8 procesadores Intel $\odot$ i7. En el enfoque mono-objetivo se ha resuelto el déficit de presión aplicando penalizaciones en el coste; ello no ha supuesto problemas en la comparación de resultados, porque en todos los casos finalmente se alcanza una solución sin déficit de presión. La naturaleza estocástica de los algoritmos genéticos obliga a realizar un análisis de la convergencia en base a criterios estadísticos; concretamente se ha considerado suficiente realizar un total de 50 repeticiones de cada escenario planteado, para concluir si los cambios en los valores promedio son estadísticamente significativos.

La resolución de problemas de optimización multiobjetivo no proporciona una solución única, sino que se genera un conjunto de soluciones no dominadas entre sí conocidas como el Frente de Pareto. La condición de no dominancia implica que, entre el conjunto de soluciones, ninguna de ellas es mejor que las otras en todos los objetivos. Por lo tanto, la elección de una solución de entre las que conforman el Frente de Pareto puede basarse en múltiples criterios. En el presente caso, se propone priorizar la calidad del servicio frente al ahorro económico y, por tanto, se elige la solución con menor déficit de presión como la mejor solución entre el Frente de Pareto.

En los escenarios de optimización que se han planteado, el algoritmo alcanza soluciones sin déficit de presión en pocas iteraciones, siendo la solución elegida, en todo caso, la que tiene el menor déficit de presión, y que se indica con un círculo en la Figura 9. Sin embargo, el Frente de Pareto sigue manteniendo las soluciones con déficit de presión y esto favorece la diversidad de la población. La Figura 10 muestra el valor de los dos objetivos considerados, para el individuo mejor posicionado al finalizar la evaluación, tras diferentes generaciones, en uno de los procesos estudiados.

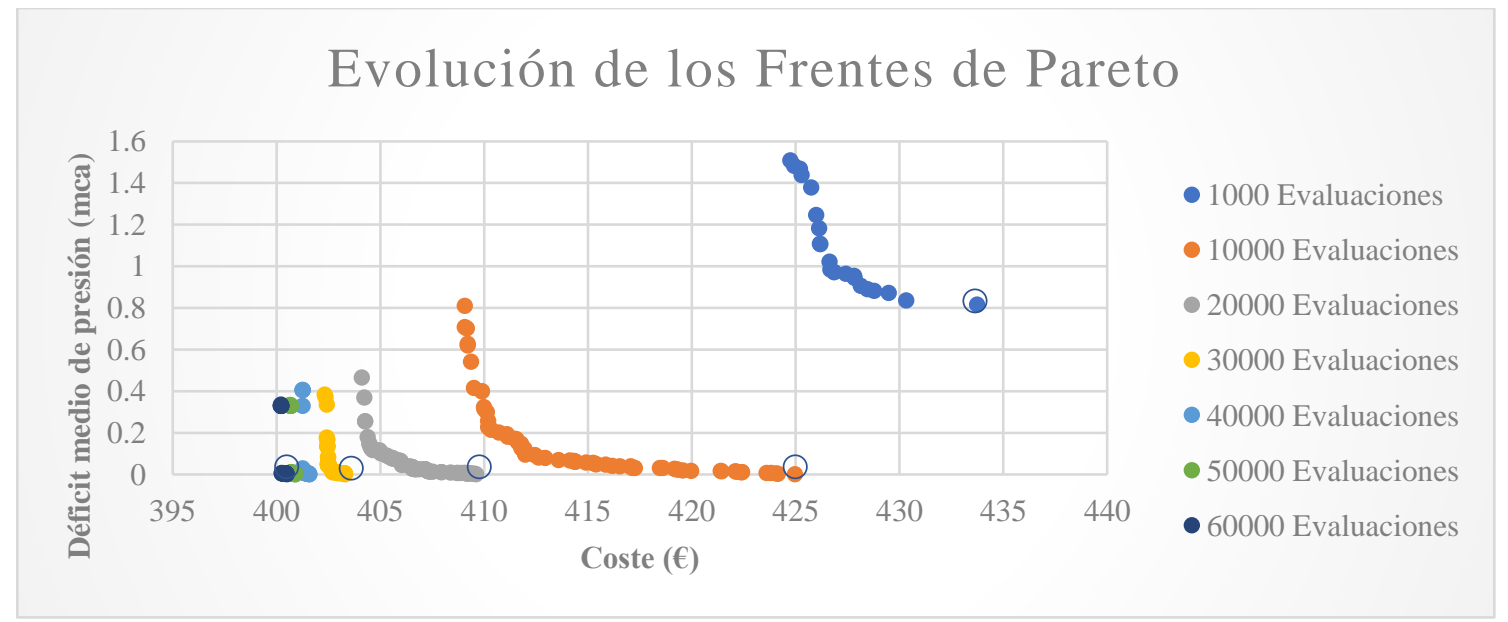

Figura 9. Representación del conjunto de soluciones no dominadas dentro del conjunto de la población al finalizar distintas generaciones. Las soluciones seleccionadas en cada frente se han remarcado con un círculo. 


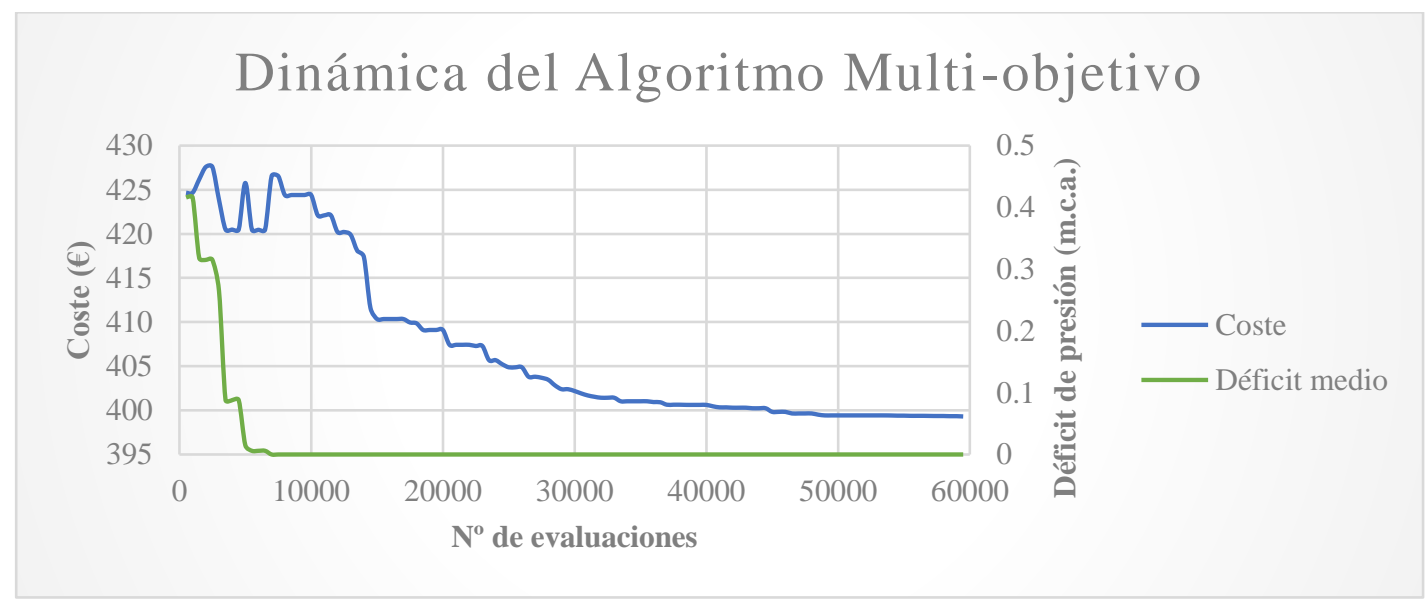

Figura 10. Valor de los dos objetivos para el individuo seleccionado del Frente de Pareto (puntos redondeados de la Figura 9) al final de la evaluación de cada generación.

A la vista de la dinámica del algoritmo se ha considerado adecuado realizar los análisis dentro del marco de las 60.000 primeras evaluaciones (120 generaciones). Los escenarios que se han analizado son los siguientes:

- Escenario A: optimización multiobjetivo con evaluación paralela de 20.000 individuos.

- Escenario B: optimización mono-objetivo con evaluación paralela de 30.000 individuos.

- Escenario C: optimización multiobjetivo con evaluación paralela de 30.000 individuos.

- Escenario D: optimización mono-objetivo con evaluación paralela de 60.000 individuos.

- Escenario E: optimización multiobjetivo con evaluación paralela de 60.000 individuos.

- Escenario F: optimización multiobjetivo con evaluación en un único hilo de 30.000 individuos.

Los escenarios se han elegido con el objetivo de disponer de suficientes casuísticas para evaluar eficazmente los resultados obtenidos a través de los distintos planteamientos. A la vista de los resultados, se concluye que los escenarios estudiados han sido suficientes para observar eficazmente esas diferencias.

Finalmente, se ha evaluado el grado de efectividad de la paralelización en la reducción del tiempo de cálculo con respecto a la aceleración máxima teórica. La aceleración máxima teórica se calcula mediante la Ley de Amdahl (Amdahl, 1967), definida por la Ecuación (27), que depende de la relación $f$ entre el tiempo de cálculo de la parte inevitablemente secuencial del programa y el tiempo de ejecución total, y del número de procesadores disponibles $N$. 


$$
S(N)=\frac{1}{f+\frac{1-f}{N}}
$$

\subsubsection{Caso de estudio. Comunidad de regantes de Peñarroya}

Las metodologías propuestas en este apartado se aplicaron a una red de riego real ubicada en Ciudad Real (España). Se implementó un módulo optimizador consistente en un conjunto de servicios web, programados en el entorno .NET y basados en la tecnología APIREST (Application Program Interface - REpresentational State Transfer) y se integró en la plataforma CORENET-COREGEST, que es la plataforma de gestión que estaba siendo utilizada en la comunidad de regantes en cuestión.

En concreto, la red de riego sobre la que se han probado las metodologías propuestas es el sector III de la comunidad de regantes de Peñarroya $\left(39^{\circ} 08^{\prime} 03.3^{\prime \prime} \mathrm{N} 3^{\circ} 06^{\prime} 17,6^{\prime \prime} \mathrm{O}\right)$, con una superficie total irrigada de 1.022 ha y 380 tomas (Figura 11).

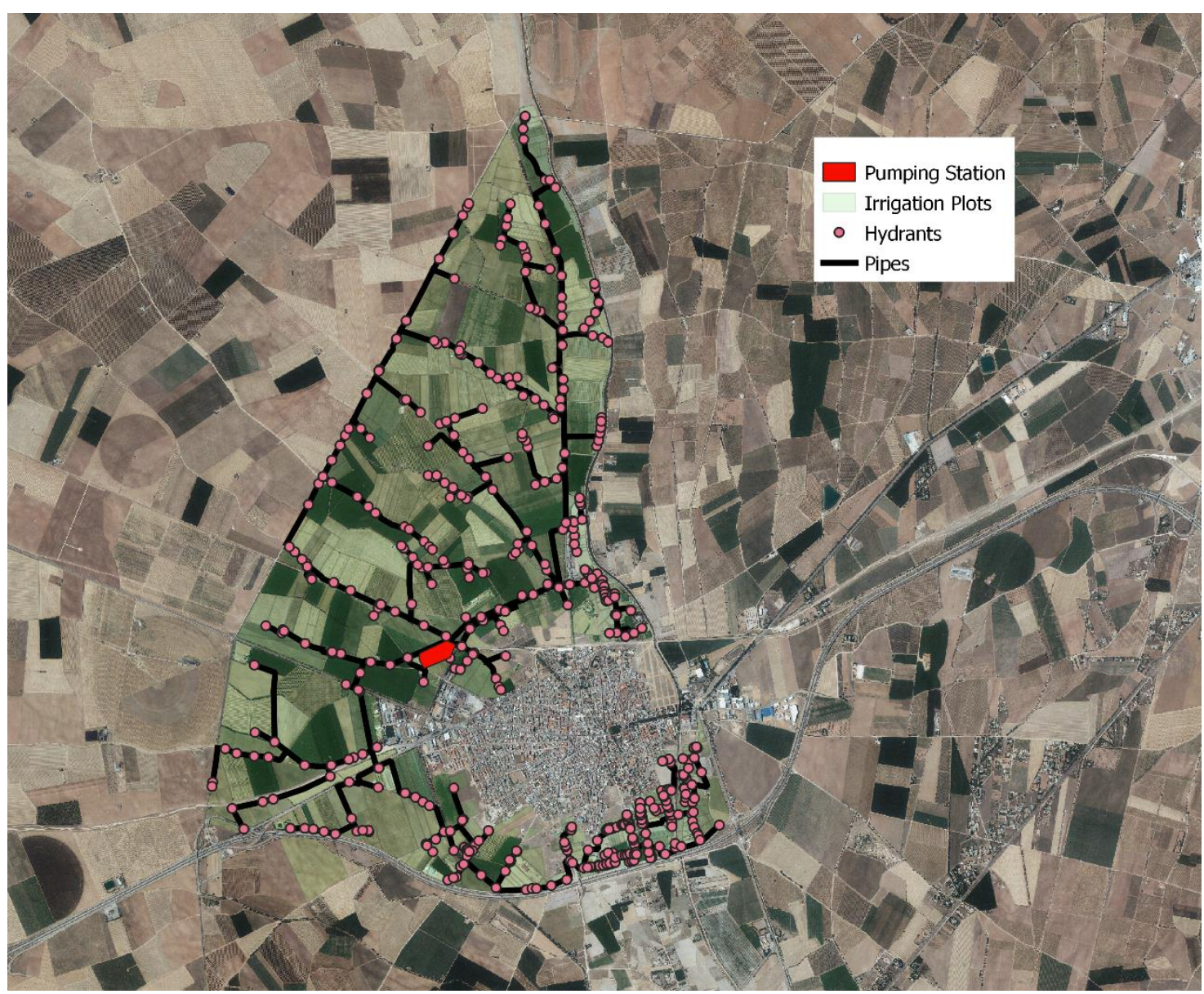

Figura 11. Esquema de la red de riego del sector III de Peñarroya. 
La estación de bombeo cuenta con 8 bombas, 7 de ellas idénticas con una potencia nominal de $189 \mathrm{~kW}$, y la otra de $55 \mathrm{~kW}$. Esta última es una bomba de velocidad variable (BVV) y solo está activa cuando el caudal demandado es muy bajo. Entre las bombas grandes, una de ellas también es de velocidad variable. La estación de bombeo está controlada por un automatismo capaz de seguir una consigna de presión de impulsión y se asume que se ha programado de acuerdo a criterios de máxima eficiencia, es decir, la combinación de bombas activas y la velocidad de las BVV se elige para entregar cualquier flujo a la presión establecida con la mejor eficiencia posible.

Debido a una serie de limitaciones mecánicas en la red de distribución (antigüedad y material de las tuberías), la presión de consigna de la estación de bombeo no puede exceder los 40 m.c.a; de lo contrario, el riesgo de roturas es demasiado alto. Así, en el estudio se ha establecido una consigna de presión de 38 m.c.a. y, para esta presión se ha calculado, de manera similar a lo descrito en el apartado 3.2.4.2, el rendimiento global de la estación para todo el rango de caudales posible (Figura 12).

Esta limitación pone de relieve las ventajas del enfoque multi-objetivo, ya que en algunos escenarios de alta demanda un cierto déficit de presión puede ser inevitable.

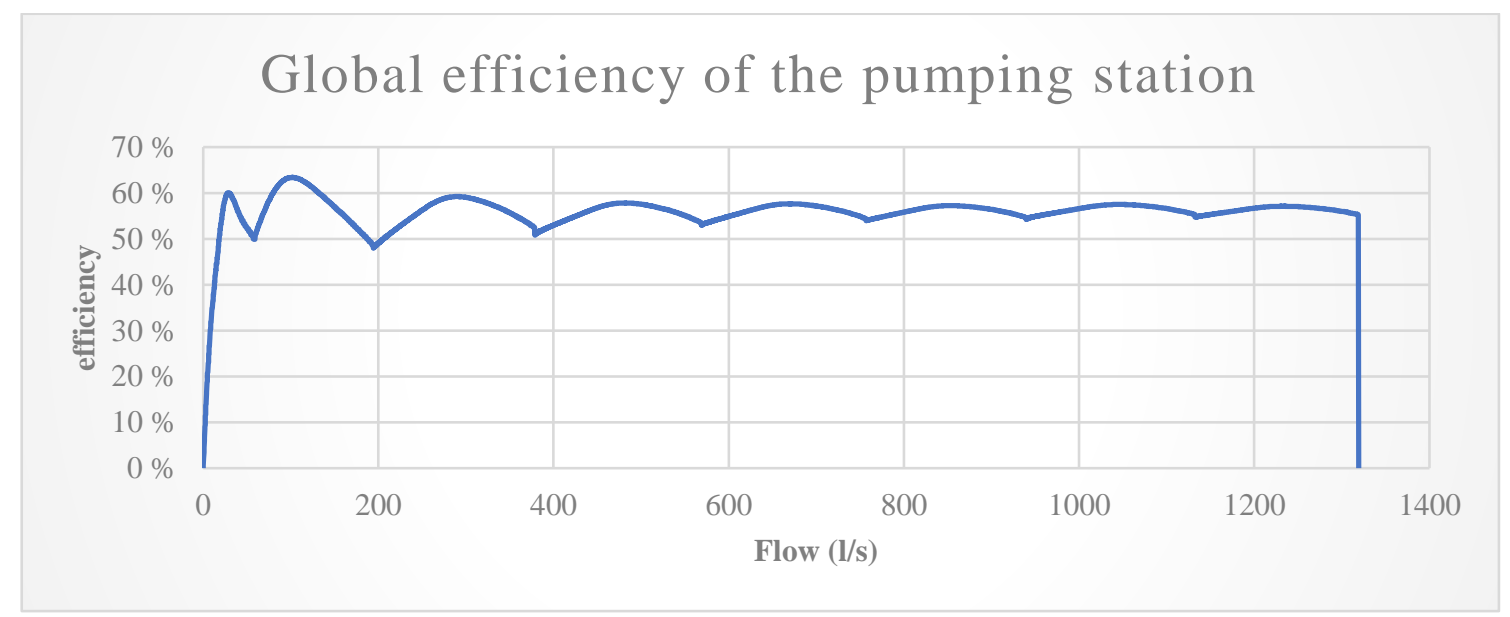

Figura 12. Curva de rendimiento global de la estación de bombeo del sector III de la comunidad de regantes del Pantano Estrecho de Peñarroya (C. Real), en función del caudal total bombeado, calculada para obtener el mejor rendimiento a partir de las curvas reales de cada bomba, con una presión de consigna de 38 m.c.a.

La tarifa eléctrica contratada por la comunidad de regantes es la 6.1A. Se trata de una tarifa con discriminación horaria de 6 períodos, donde P6 es la más barata y P1 la más cara. En la Tabla 5 se muestra la distribución de los 6 periodos horarios a lo largo del año. La comunidad de regantes tiene una potencia contratada de $50 \mathrm{~kW}$ para los períodos $\mathrm{P} 1$ y $\mathrm{P} 2$, $850 \mathrm{~kW}$ para P3 a P5 y $900 \mathrm{~kW}$ para P6. La jornada de riego analizada cuenta con 78 
peticiones con un tiempo de riego fijo cada una, lo que supone un volumen total de $33.823 \mathrm{~m}^{3}$ a entregar en un tiempo máximo de 24 horas.

Tabla 5. Distribución anual de los periodos horarios para la tarifa $6.1 \mathrm{~A}$

\begin{tabular}{|c|c|c|c|c|c|c|c|c|c|c|c|c|c|}
\hline Hora & Ene & $\mathrm{Feb}$ & Mar & Abr & May & Jun 1 & Jun2 & Jul & Ago & Sep & Oct & Nov & Dic \\
\hline 00:00 & P6 & P6 & P6 & P6 & P6 & P6 & P6 & P6 & P6 & P6 & P6 & P6 & P6 \\
\hline 01:00 & P6 & P6 & P6 & P6 & P6 & P6 & P6 & P6 & P6 & P6 & P6 & P6 & P6 \\
\hline 02:00 & P6 & P6 & P6 & P6 & P6 & P6 & P6 & P6 & P6 & P6 & P6 & P6 & P6 \\
\hline 03:00 & P6 & P6 & P6 & P6 & P6 & P6 & P6 & P6 & P6 & P6 & P6 & P6 & P6 \\
\hline 04:00 & P6 & P6 & P6 & P6 & P6 & P6 & P6 & P6 & P6 & P6 & P6 & P6 & P6 \\
\hline 05:00 & P6 & P6 & P6 & P6 & P6 & P6 & P6 & P6 & P6 & P6 & P6 & P6 & P6 \\
\hline 06:00 & P6 & P6 & P6 & P6 & P6 & P6 & P6 & P6 & P6 & P6 & P6 & P6 & P6 \\
\hline 07:00 & P6 & P6 & P6 & P6 & P6 & P6 & P6 & P6 & P6 & P6 & P6 & P6 & P6 \\
\hline 08:00 & $\mathrm{P} 2$ & P2 & P4 & P5 & P5 & P4 & $\mathrm{P} 2$ & $\mathrm{P} 2$ & P6 & P4 & P5 & $\mathrm{P} 4$ & $\mathrm{P} 2$ \\
\hline 09:00 & P2 & $\mathrm{P} 2$ & P4 & P5 & P5 & P3 & $\mathrm{P} 2$ & $\mathrm{P} 2$ & P6 & P3 & P5 & P4 & P2 \\
\hline 10:00 & P1 & P1 & P4 & P5 & P5 & P3 & $\mathrm{P} 2$ & $\mathrm{P} 2$ & P6 & P3 & P5 & P4 & P1 \\
\hline 11:00 & P1 & $\mathrm{P} 1$ & P4 & P5 & P5 & P3 & $\mathrm{P} 1$ & $\mathrm{P} 1$ & P6 & P3 & P5 & P4 & $\mathrm{P} 1$ \\
\hline $12: 00$ & P1 & P1 & P4 & P5 & P5 & P3 & $\mathrm{P} 1$ & P1 & P6 & P3 & P5 & P4 & P1 \\
\hline $13: 00$ & $\mathrm{P} 2$ & P2 & P4 & P5 & P5 & P3 & $\mathrm{P} 1$ & $\mathrm{P} 1$ & P6 & P3 & P5 & P4 & $\mathrm{P} 2$ \\
\hline $14: 00$ & $\mathrm{P} 2$ & P2 & P4 & P5 & P5 & P3 & $\mathrm{P} 1$ & $\mathrm{P} 1$ & P6 & P3 & P5 & $\mathrm{P} 4$ & $\mathrm{P} 2$ \\
\hline 15:00 & $\mathrm{P} 2$ & $\mathrm{P} 2$ & P4 & P5 & P5 & $\mathrm{P} 4$ & $\mathrm{P} 1$ & $\mathrm{P} 1$ & P6 & $\mathrm{P} 4$ & P5 & $\mathrm{P} 4$ & $\mathrm{P} 2$ \\
\hline $16: 00$ & $\mathrm{P} 2$ & P2 & P3 & P5 & P5 & $\mathrm{P} 4$ & $\mathrm{P} 1$ & $\mathrm{P} 1$ & P6 & $\mathrm{P} 4$ & P5 & P3 & $\mathrm{P} 2$ \\
\hline $17: 00$ & $\mathrm{P} 2$ & P2 & P3 & P5 & P5 & $\mathrm{P} 4$ & $\mathrm{P} 1$ & $\mathrm{P} 1$ & P6 & P4 & P5 & P3 & $\mathrm{P} 2$ \\
\hline $18: 00$ & P1 & $\mathrm{P} 1$ & P3 & P5 & P5 & $\mathrm{P} 4$ & $\mathrm{P} 1$ & $\mathrm{P} 1$ & P6 & $\mathrm{P} 4$ & P5 & P3 & $\mathrm{P} 1$ \\
\hline 19:00 & P1 & $\mathrm{P} 1$ & P3 & P5 & P5 & $\mathrm{P} 4$ & $\mathrm{P} 2$ & $\mathrm{P} 2$ & P6 & $\mathrm{P} 4$ & P5 & P3 & $\mathrm{P} 1$ \\
\hline 20:00 & $\mathrm{P} 1$ & P1 & P3 & P5 & P5 & $\mathrm{P} 4$ & $\mathrm{P} 2$ & $\mathrm{P} 2$ & P6 & $\mathrm{P} 4$ & P5 & $\mathrm{P} 3$ & P1 \\
\hline $21: 00$ & $\mathrm{P} 2$ & P2 & P3 & P5 & P5 & P4 & $\mathrm{P} 2$ & $\mathrm{P} 2$ & P6 & P4 & P5 & P3 & P2 \\
\hline $22: 00$ & $\mathrm{P} 2$ & P2 & P4 & P5 & P5 & P4 & $\mathrm{P} 2$ & $\mathrm{P} 2$ & P6 & P4 & P5 & $\mathrm{P} 4$ & $\mathrm{P} 2$ \\
\hline 23:00 & $\mathrm{P} 2$ & P2 & P4 & P5 & P5 & $\mathrm{P} 4$ & $\mathrm{P} 2$ & P2 & P6 & $\mathrm{P} 4$ & P5 & $\mathrm{P} 4$ & $\mathrm{P} 2$ \\
\hline
\end{tabular}




\subsubsection{Resultados}

\subsubsection{Comparación entre escenarios}

Para cada escenario, tal como se han descrito anteriormente, se han realizado 50 ensayos independientes. Los resultados se resumen en la Tabla 6 y se muestran en la Figura 13. El gráfico de caja y bigotes sugiere que las diferencias entre escenarios con respecto al coste mínimo alcanzado son significativas, a excepción de los escenarios $\mathrm{C}$ y F, lo que no es sorprendente ya que en ambos casos se trata de una optimización multiobjetivo con 30.000 evaluaciones, siendo la única diferencia entre ellos que el cálculo se paralelice o no. Es decir, la evaluación paralela de la función objetivo no afecta a la tasa de convergencia del algoritmo, sino que únicamente acelera el proceso al aumentar la capacidad de cálculo, por lo que, a igual número de evaluaciones de la función objetivo, los resultados en cuanto a coste esperado son los mismos.

También se puede concluir que los escenarios correspondientes a la optimización multi-objetivo de 20.000 individuos (A) y la mono-objetivo de 30.000 (B) son bastante similares.

Tabla 6. Resumen de estadísticos para el conjunto de escenarios planteados.

\begin{tabular}{llllll}
\hline Escenario & $\mathrm{N}^{\mathrm{o}}$ Ensayos & $\begin{array}{l}\text { Media } \\
\text { Objetivo }\end{array}$ & $\begin{array}{l}\text { Desviación } \\
\text { Típica }\end{array}$ & $\begin{array}{l}\text { Media } \\
\text { Tiempo }\end{array}$ & $\begin{array}{l}\text { Desviación } \\
\text { Típica }\end{array}$ \\
\hline & & $(€)$ & $(€)$ & $(\mathrm{s})$ & $(\mathrm{s})$ \\
$\mathrm{A}$ & 50 & 409,25 & 3,36 & 62,54 & 1,45 \\
$\mathrm{~B}$ & 50 & 408,96 & 2,98 & 95,10 & 2,79 \\
$\mathrm{C}$ & 50 & 403,82 & 2,25 & 89,60 & 3,55 \\
$\mathrm{D}$ & 50 & 406,21 & 2,83 & 193,06 & 5,55 \\
$\mathrm{E}$ & 50 & 400,66 & 1,94 & 179,28 & 4,71 \\
$\mathrm{~F}$ & 50 & 404,67 & 1,99 & 418,21 & 14,03 \\
\hline
\end{tabular}

La apreciación subjetiva se ha corroborado realizando la prueba-t (Student, 1908) para la comparación de los valores medios de coste, ya que es la prueba más extendida y aceptada para comparar muestras independientes cuando el estadístico sigue una distribución normal. En caso de que los datos fueran notablemente no normales, la prueba $\mathrm{t}$ daría resultados inexactos. Sin embargo, el conjunto de datos tratado ha cumplido las condiciones de normalidad. (Student, 1908) 


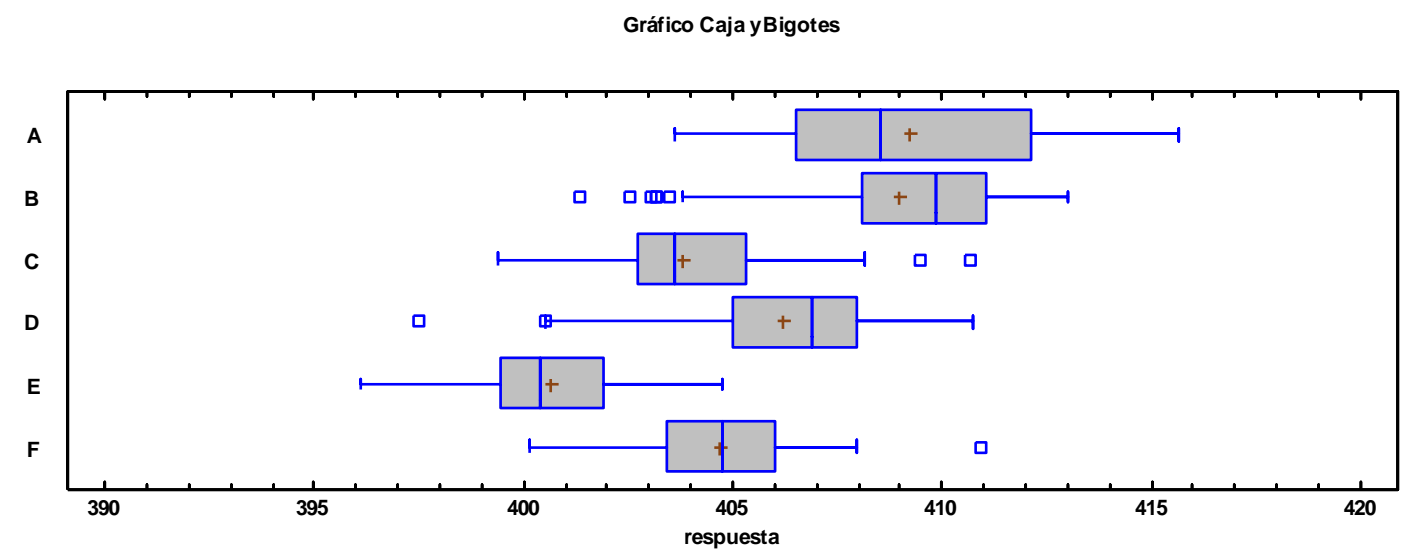

Figura 13. Representación de los datos del valor objetivo mediante el gráfico de caja y bigotes.

Tal como sugería el gráfico de caja y bigotes, el P-valor para las comparaciones A con $\mathrm{B}$ y $\mathrm{C}$ con $\mathrm{F}$ es superior a 0,05 , por lo tanto, se acepta la hipótesis nula y se considera con un $95 \%$ de probabilidad que las medias no han cambiado. En el resto de comparaciones el P-valor de la prueba-t es inferior a 0,05 y, por consiguiente, las diferencias en los valores medios se consideran estadísticamente significativas.

Una vez realizadas estas pruebas se está en condiciones de afirmar que el planteamiento del problema de optimización mediante un enfoque multiobjetivo alcanza mejores resultados en un número menor de iteraciones. Como ejemplos concretos, comparando el escenario A con el B, mediante el enfoque multiobjetivo se ha alcanzado un valor igual con un $33 \%$ menos de evaluaciones. Comparando el escenario B con C y el D con el $\mathrm{E}$ se concluye que, a igual número de evaluaciones, con el planteamiento multiobjetivo se ha alcanzado un resultado mejor. Por último, quizás la comparación que más evidencia la mejora respecto al enfoque mono-objetivo es el caso $\mathrm{C}$ con el $\mathrm{D}$, ya que la solución es significativamente mejor con el multiobjetivo en tan solo la mitad de evaluaciones.

Por lo que respecta al tiempo de cálculo, aunque se ha constatado una leve mejora del multiobjetivo frente al mono-objetivo a igualdad de evaluaciones, esta reducción es muy inferior a las conseguidas mediante la paralelización del algoritmo, así como las que se pueden conseguir gracias a la reducción del número total de iteraciones para alcanzar la convergencia.

Para obtener la relación $f$ entre el tiempo de computación de la parte inevitablemente secuencial del programa y el tiempo total de cómputo, la parte inevitablemente secuencial 
del código debe de ser identificada en primer lugar. Una de las ventajas de los algoritmos de optimización basados en poblaciones es que la evaluación de cada cromosoma dentro de una generación es independiente del resto. Por lo tanto, la parte del código correspondiente a la evaluación de cada generación es susceptible de ser paralelizada. Una medida aproximada del tiempo de computación correspondiente al código susceptible de ser paralelizado puede tomarse como el $95 \%$ del tiempo total de computación. Esto significa que la aceleración máxima ideal con recursos ilimitados sería:

$$
\lim _{N \rightarrow \infty} S(N)=\frac{1}{0.05}=20
$$

Sin embargo, la dependencia entre distintas generaciones del algoritmo genético limita el número máximo de hilos de ejecución al tamaño de la población. Como en este estudio se ha trabajado con un tamaño de población de 500 cromosomas, la aceleración máxima teórica sería de:

$$
S(500)=\frac{1}{0.05+0.95 / 500}=19.27
$$

Puesto que el análisis se ha ejecutado en un ordenador personal con ocho procesadores, la aceleración máxima teórica se limita a:

$$
S(8)=\frac{1}{0.05+0.95 / 8}=5.93
$$

En realidad, esta aceleración ideal no puede alcanzarse debido a tareas internas de organización de las tareas, reparto de cargas o por los costes de comunicación (Grama et al., 2003). La comparación entre los escenarios C y F muestra que la aceleración real alcanzada gracias a la paralelización de la evaluación de la función objetivo es:

$$
S=\frac{T(F)}{T(C)}=\frac{418.21}{89.60}=4.67
$$

Esto indica que la eficiencia real de la paralelización que se ha obtenido ha sido del 80\% aproximadamente, en comparación con la aceleración máxima teórica. Debe remarcarse que, teóricamente, todavía existe margen de mejora del tiempo de computación sencillamente incrementando el número de procesadores, aunque en tal caso sería probable que la eficiencia real pudiese empeorar.

Otra conclusión con respecto a la paralelización del algoritmo tiene que ver con el efecto de escalar el problema. Como predijo la observación de Gustafson a la Ley de Amdahl 
(Gustafson, 1988), la relación entre la parte inevitablemente secuencial del programa y la parte paralelizable podría reducirse a medida que el problema se escala. En el actual caso de estudio, se ha medido el ratio $f$ para distinto número de evaluaciones de la función objetivo. Los resultados mostrados en la Figura 14 confirman esa predicción. Se puede concluir que los beneficios potenciales de paralelizar el programa son mayores a medida que se escala el problema.

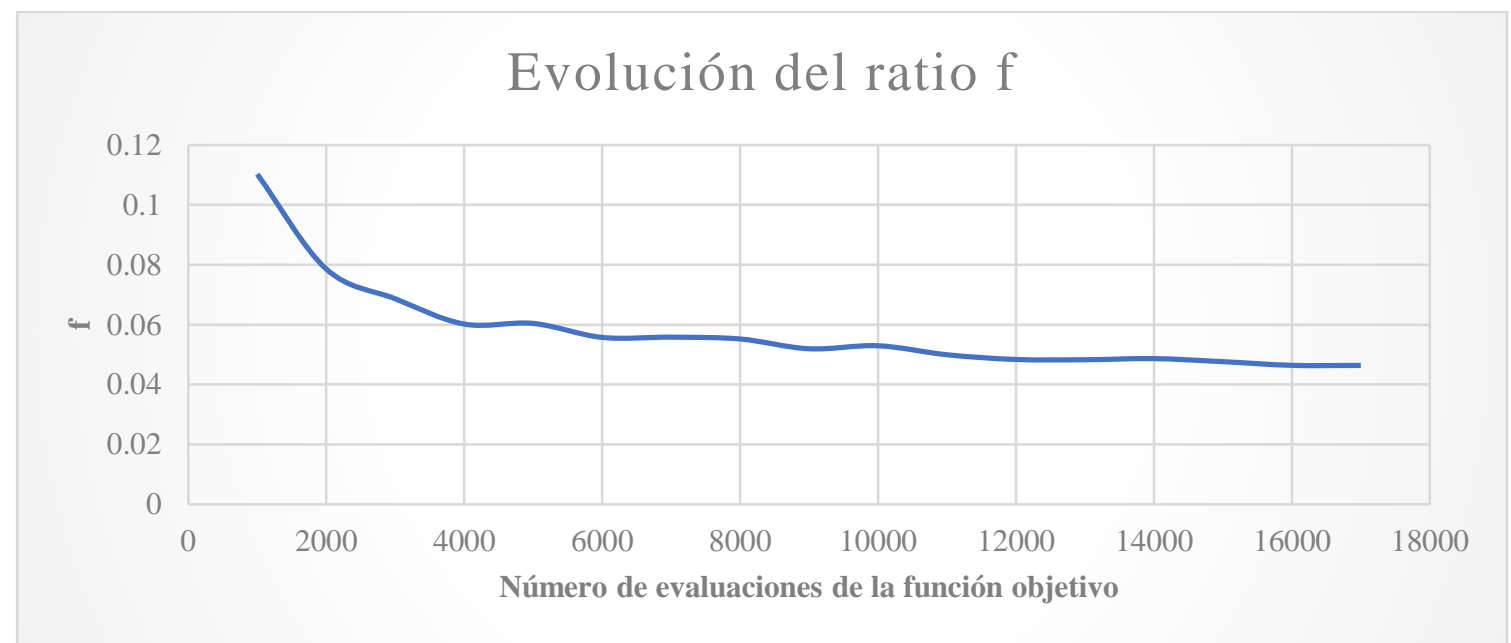

Figura 14. Valor del ratio entre el tiempo de computación de la parte inevitablemente secuencial del programa y el tiempo total de cómputo

Finalmente, respecto a la mejora alcanzada por el algoritmo en cuanto al valor de los objetivos en comparación con la programación inicial de riego llevada a cabo por la comunidad de regantes, la Tabla 7 resume los principales indicadores que se han obtenido en una de las soluciones correspondientes al escenario $\mathrm{C}$.

Tabla 7. Resumen de la mejora alcanzada por una de las soluciones respecto a la programación de riego real.

\begin{tabular}{lll}
\hline & Escenario inicial & Solución propuesta \\
\hline $\mathrm{N}^{\mathrm{o}}$ de hidrantes & 78 & 78 \\
Volumen total $\left(\mathrm{m}^{3}\right)$ & $33.823,28$ & $33.823,28$ \\
Energía consumida $(\mathrm{kWh})$ & $6.302,22$ & $6.072,3$ \\
Coste total $(€)$ & 428,44 & 402,69 \\
Penalización E.P. $(€)$ & 0 & 0 \\
Déficit medio de presión (m.c.a.) & 0,87 & 0 \\
Presión hidrante crítico (m.c.a.) & 16,43 & 25,38 \\
$\mathrm{~N}^{\mathrm{o}}$ de hidrantes con presión < 25 m.c.a. & 4 & 0 \\
\hline
\end{tabular}




\subsubsection{Conclusiones}

En el presente apartado se ha propuesto una nueva formulación para optimizar el programa de riego a partir de un conjunto de peticiones prestablecidas, contemplando tanto el término de energía como la penalización por exceso de potencias para los diferentes periodos tarifarios. Por otra parte, se ha propuesto un algoritmo de optimización mejorado para resolver el problema de obtener la programación de riego óptima desde un punto de vista energético y de calidad del servicio simultáneamente.

Uno de los objetivos fundamentales ha sido el de reducir el esfuerzo computacional requerido por el algoritmo. En este ámbito, se ha demostrado mediante el análisis de una serie de casos que el planteamiento multiobjetivo permite alcanzar la convergencia en un número menor de evaluaciones, hasta un 50\% menos para alcanzar el mismo resultado, y que la paralelización de dicho cálculo utilizando procesadores multi-core, hoy en día habituales en cualquier PC, consigue reducir aproximadamente un $80 \%$ el tiempo de computación. Debe mencionarse también que, para el caso de estudio, se ha conseguido una reducción del coste en energía del 6-7\% con ausencia de déficits de presión en las tomas activas.

Se ha conseguido, en definitiva, desarrollar un algoritmo capaz de entregar una solución óptima en pocos minutos, convirtiéndolo en una solución viable para optimizar las peticiones diarias de agua. El optimizador lo componen una serie de servicios web programados en el entorno .NET utilizando la tecnología API-REST, lo que facilita su integración en cualquier plataforma de gestión de comunidades de regantes.

Con respecto a la magnitud del problema, la complejidad hidráulica de las redes de riego puede variar, aunque el caso de estudio elegido podría considerarse representativo. La metodología propuesta es adecuada para redes de igual o menor tamaño. Se necesitarían más mejoras y nuevos enfoques para abordar la optimización de la programación del riego en tiempo real en redes más complejas.

Además, debido a los beneficios de combinar el uso de energía eléctrica convencional con fuentes renovables, como la fotovoltaica o la eólica, el desarrollo de algoritmos más sofisticados sería de gran interés en futuras investigaciones para la gestión eficiente del riego. 


\subsection{Mejoras en el método de maximización del volumen entregado por gravedad}

Las conclusiones que se extraen del estudio de la metodología descrita en el apartado 3.2 evidencian la exigencia del desarrollo de nuevos enfoques o cambios de paradigma que mejoren sustancialmente la eficiencia computacional, si el objetivo que se persigue es el desarrollo de herramientas que sirvan de apoyo a la toma de decisiones en la operación diaria de la red.

En el apartado anterior se han propuesto dos mejoras que ayudan a acelerar el algoritmo general de optimización, siendo de aplicabilidad tanto para el método de minimización del consumo energético (bombeo) como para el de maximización del volumen entregado en un periodo de tiempo determinado (gravedad). Además, dichas mejoras se han probado con éxito en un caso de estudio real.

En el presente apartado se introduce una mejora que se aplica específicamente al método de maximización del volumen entregado por gravedad. Se parte del mismo problema a resolver que el descrito en el apartado 3.2.3, con las mismas restricciones y condiciones, pero con un planteamiento distinto para su resolución.

\subsubsection{Descripción del método}

Mediante esta variante, el algoritmo genético optimiza un único intervalo de tiempo, y se ejecuta tantas veces como sea necesario hasta completar el periodo de riego por gravedad. Se trata de una optimización secuencial, en la que los resultados que se obtienen en cada instante tienen influencia en los resultados de los instantes posteriores.

Se respeta la condición de que cuando una toma ha iniciado el riego, continúa abierta en los sucesivos intervalos de cálculo hasta que finaliza su tiempo de riego.

En la variante propuesta, el tipo de variable a utilizar para configurar el cromosoma va a ser una variable binaria, en lugar de entera. Para cada instante de tiempo la variable indica si la toma está abierta (1) o cerrada (0). Esta característica simplifica el algoritmo de optimización porque reduce en gran medida el espacio de soluciones, pero significa un incremento en la complejidad de la implementación, debido a que en cada iteración se ha de tener en cuenta el estado anterior a la hora de calcular el nuevo intervalo, así como el hecho 
de que el número de variables se va a ir reduciendo progresivamente, a medida que las tomas se van activando y completando su intervalo de riego.

De un modo conciso, el esquema de funcionamiento es el siguiente. Se maximiza el caudal para el primer instante de tiempo utilizando como variables todas las tomas con diferencia de cota respecto de la balsa mayor a la presión mínima requerida. El siguiente intervalo de tiempo y los sucesivos repetirán escenario de consumo, es decir, las tomas abiertas durante el primer intervalo permanecerán abiertas, hasta que al menos una de ellas finalice su tiempo de riego.

En el intervalo de tiempo coincidente con la finalización del riego de alguna de las tomas, se lanza por segunda vez el algoritmo genético, pero esta vez disminuye el número de variables, ya que dejan de formar parte del conjunto aquellos riegos que ya se han iniciado, aunque no hayan finalizado todavía. Hay que tener en cuenta que, para el cálculo hidráulico de la red, deben estar activas todas las tomas que iniciaron el riego en el cálculo anterior, excepto las que hayan finalizado. El resultado de la optimización serán los riegos adicionales que se pueden añadir a los riegos activos, en caso de que cumpliesen la restricción de presión.

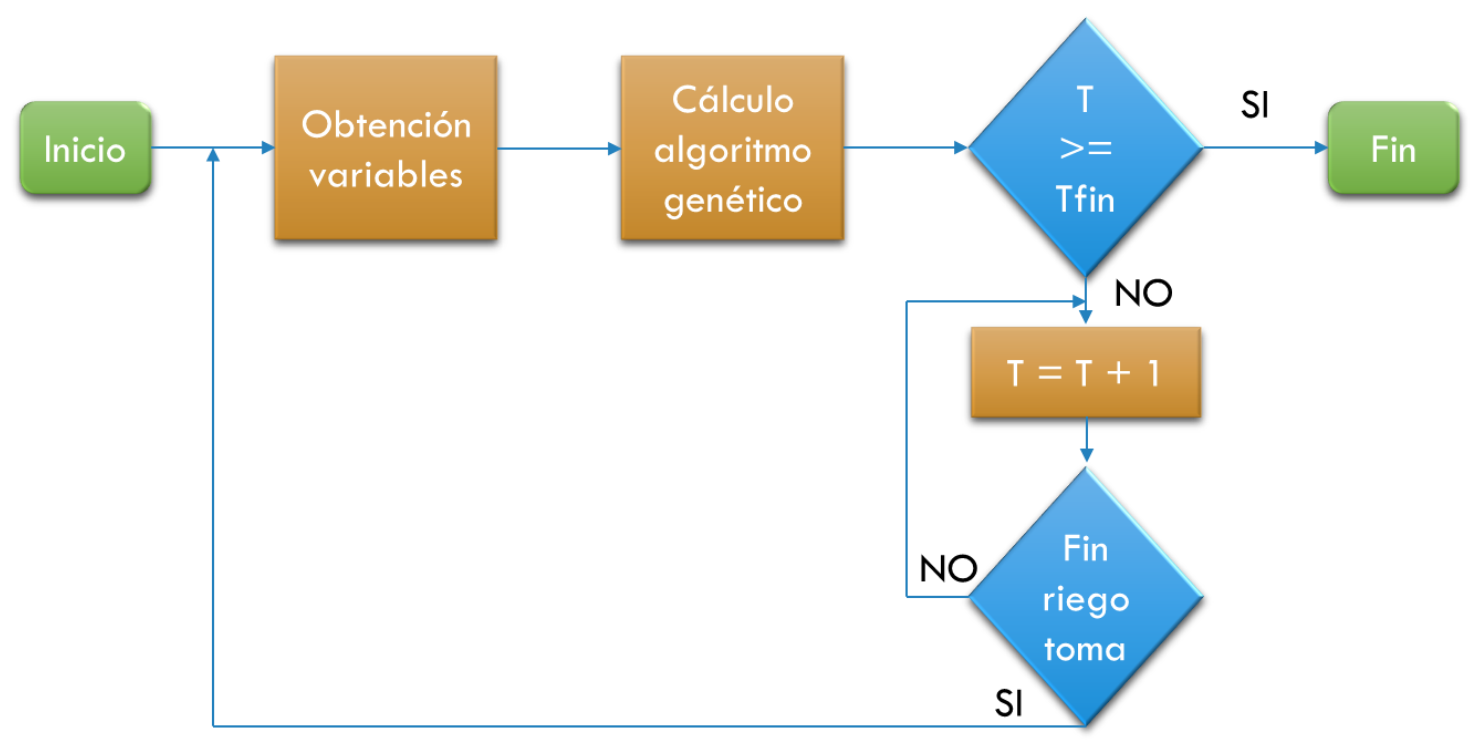

Figura 15. Diagrama de flujo del algoritmo para la maximización del volumen entregado por gravedad.

Llegados a este punto se sigue la misma estrategia: el escenario de consumo se repite en los sucesivos instantes hasta que alguna de las tomas finalice el riego, y el proceso general sigue así hasta que concluye el periodo de riego por gravedad o hasta que se eliminan todas las variables en el problema de optimización porque todos los riegos se han iniciado. 


\subsubsection{Resultados de la aplicación de la variante al caso de estudio de "El Realón"}

La variante propuesta se ha aplicado en la red de riego del Realón, descrita en apartado 3.2.4. Como recordatorio, la red cuenta con 62 hidrantes multiusuario con un total de 362 tomas y una balsa de regulación en cabecera. La presión mínima requerida en todas las tomas era de $25 \mathrm{~m}$.

El número de tomas cuya diferencia de cota con la balsa es mayor de 25 m es de 191 . Este número coincide con el tamaño del conjunto inicial de variables. El periodo de tiempo destinado al riego sin aporte energético es de 14 horas (de 10:00 a 24:00), y se ha discretizado en intervalos de 5 minutos.

Los resultados presentados en la Figura 16 corresponden al análisis de la programación actual. Se concluye que la presión mínima de servicio en cada punto de consumo (25 m.c.a.) en general no está garantizada en todo momento. En total hay 20 tomas que presentan una presión inferior a la mínima de servicio. Por otra parte, el volumen total entregado a lo largo de las 14 horas de riego sin aporte de energía es de $1.635 \mathrm{~m}^{3}$.

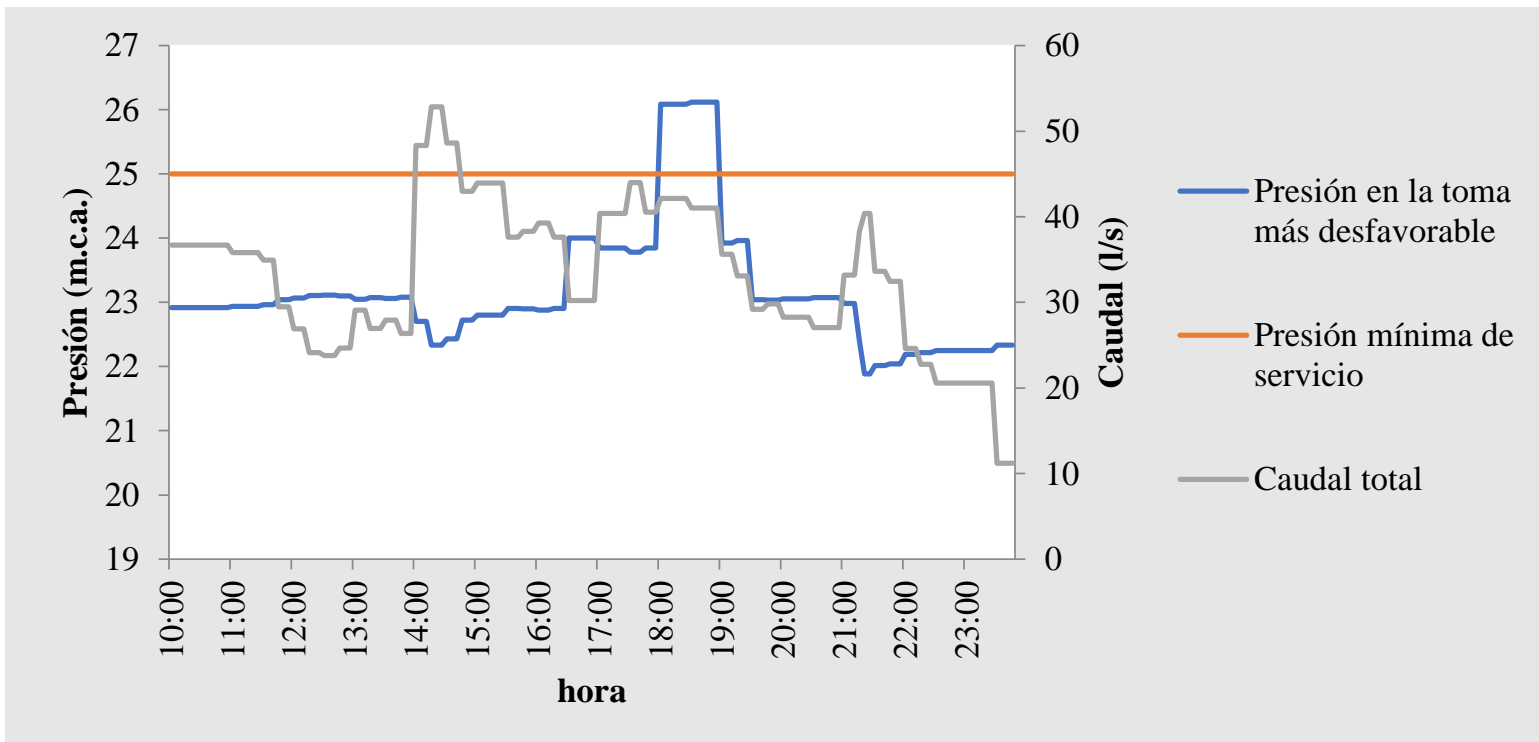

Figura 16. Resultados de simular la programación de riego actual.

En cuanto a los resultados obtenidos siguiendo la metodología definida en el apartado 3.2.3 (Figura 17), se ha alcanzado un suministro de $2.514 \mathrm{~m}^{3}$. Se observa que todas las tomas 
activas presentan una presión igual o superior a la mínima de servicio. El tiempo de cálculo de la solución presentada es del orden de 2 - 3 horas ${ }^{2}$.

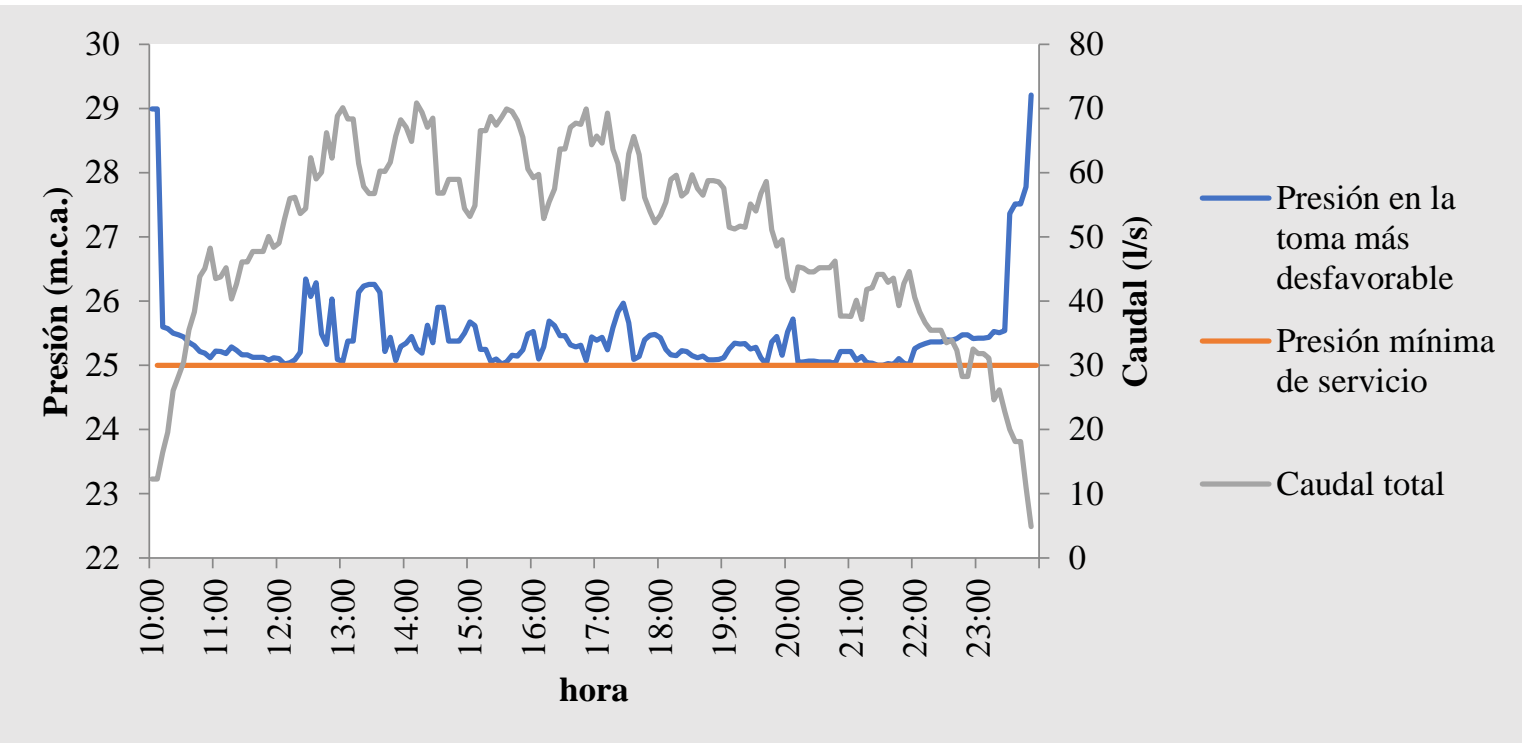

Figura 17. Resultados optimización mediante la metodología descrita en apartado 3.2.3

Siguiendo el método expuesto en el presente apartado se ha logrado suministrar un volumen de 2.517,2 $\mathrm{m}^{3}$ a lo largo de las 14 horas de riego sin bombeo. Este valor supone un aumento del 53,95\% respecto de la situación real de partida, y un ligero aumento respecto del resultado obtenido mediante el método anterior. Se observa en los resultados que, con la metodología anterior el caudal total resulta más homogéneo a lo largo de la jornada (Figura 17), mientras que la nueva metodología (Figura 18) tiende a concentrar mayores caudales al principio del periodo de optimización.

El volumen máximo teórico que podría suministrarse por gravedad es de $2.673 \mathrm{~m}^{3} \mathrm{si}$ todas las tomas con suficiente cota completasen el tiempo de riego peticionado, por lo tanto, se ha alcanzado un $94,1 \%$ del máximo potencial.

\footnotetext{
${ }^{2}$ Cálculos realizados con un procesador Intel Core 2 Duo a $2 \mathrm{GHz}$.
} 


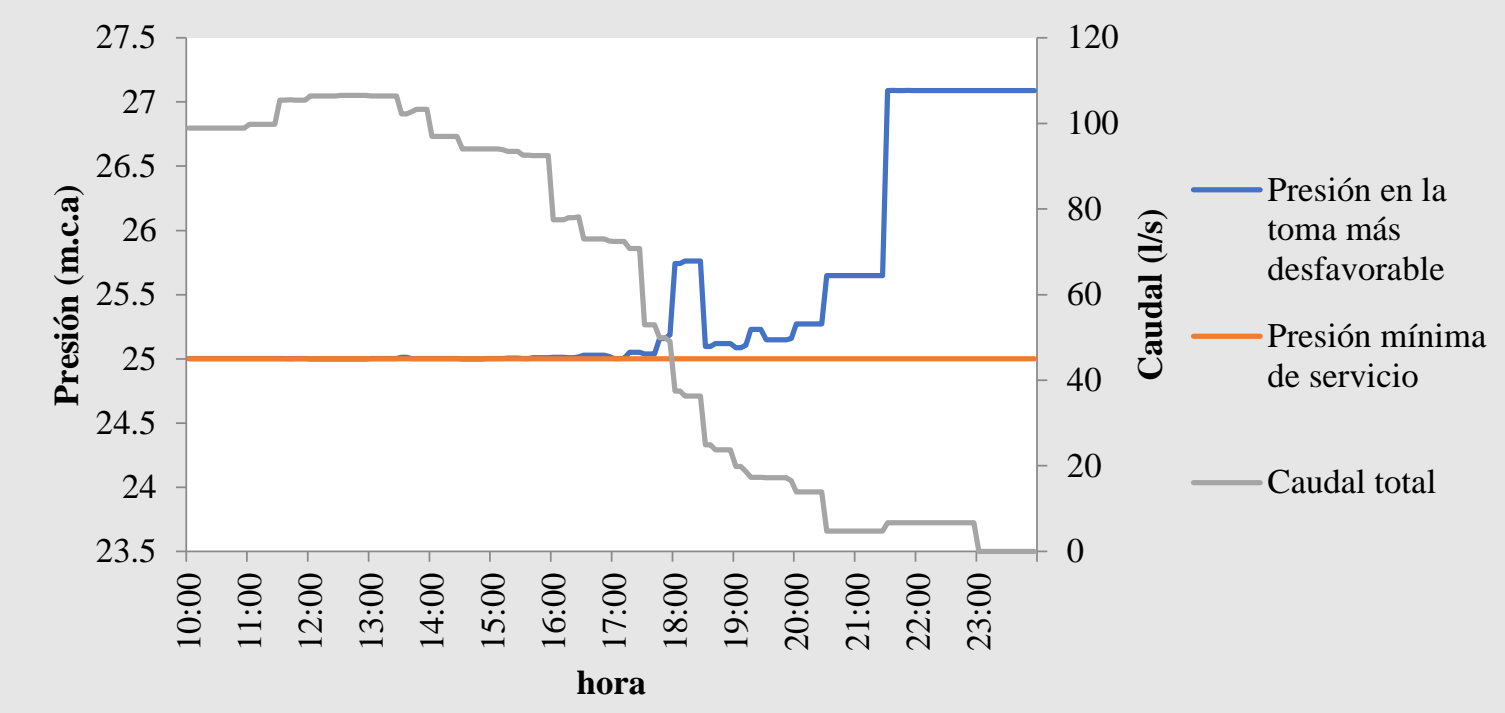

Figura 18. Resultados optimización mediante la nueva metodología.

La importancia del desarrollo de métodos para la maximización del volumen entregado por gravedad reside en el hecho de que un aumento en este volumen conlleva una reducción del volumen bombeado durante las horas nocturnas, y esto se puede traducir en un ahorro energético. A su vez, la reducción del caudal bombeado puede permitir una disminución en la presión de consigna debido a las menores pérdidas de carga en la red. Finalmente, se puede obtener aún otro ahorro adicional si se consigue que la estación de bombeo trabaje en todo momento en puntos de funcionamiento eficientes.

Además, mediante esta metodología se ha garantizado en todo momento la presión mínima en las tomas activas.

Por otra parte, si se analiza la curva de caudal total se puede concluir que las tomas que se activan en último lugar son las más restrictivas en cuanto a la penalización de la presión de funcionamiento. Por lo tanto, la presente metodología aporta información acerca de los puntos críticos de la red.

El tiempo de cálculo empleado para optimizar el riego por gravedad ha sido inferior a 15 minutos. Esto supone dividir por 10 el tiempo necesario mediante la anterior metodología, con lo que el nuevo método podría ser de aplicabilidad en la operación de redes en tiempo real. 


\subsubsection{Conclusiones al método mejorado para maximizar el volumen por gravedad}

Se ha desarrollado una metodología para la obtención de una programación de riego que maximiza el volumen total entregado sin aporte de energía en un determinado intervalo de tiempo, a la vez que garantiza una presión mínima en las tomas activas.

Siguiendo el método expuesto se han obtenido resultados para dos casos de estudio que incrementan el volumen entregado alrededor de un 30\%, presentando presiones de trabajo mayores que la deseada en cada instante.

Con esta nueva metodología se ha logrado reducir el tiempo de cálculo a una magnitud que lo hace adecuado para la optimización en tiempo real de redes de riego.

Los resultados aportados por el algoritmo ofrecen implícitamente información sobre los puntos más críticos de la red.

\subsection{Conclusiones generales}

Los sistemas de bombeo incurren en uno de los costes de operación más importantes de los sistemas de riego. Por ello, se ha tratado de investigar las mejores soluciones que permitan alcanzar una reducción sustancial en el consumo y el coste en energía, siempre que se garantice la calidad del suministro de agua.

En trabajos anteriores se ha demostrado que, en los sistemas de bombeo por inyección directa, en los que la estación de bombeo cuenta con al menos una bomba de velocidad variable y mantiene una presión de consigna constante a la salida, una reorganización temporal de las demandas de agua de cada parcela puede conducir a una reducción en el consumo energético gracias a la posibilidad de ajustar la presión de consigna a un valor mínimo, por un lado, y por otro conseguir que la estación de bombeo funcione en todo momento en los puntos de mejor rendimiento.

El problema de optimización que debe plantearse para obtener los instantes más convenientes para abrir cada toma de riego es muy complejo, siendo los métodos de optimización metaheurísticos los más adecuados para su resolución.

Sin embargo, una de las desventajas de estos métodos de optimización es que requieren, en general, un número elevado de evaluaciones de la función objetivo hasta alcanzar la convergencia. Esto se traduce en tiempos de computación que exceden el umbral aceptable para considerar que la optimización responde a los requisitos de la operación diaria. 
En consecuencia, para que las metodologías sean de aplicabilidad en el contexto de la operación en "tiempo real", se precisa mejorar la convergencia de los algoritmos de partida.

En este capítulo se ha propuesto una mejora del método general de optimización mediante la cual se ha conseguido reducir el tiempo de convergencia a través de dos actuaciones: por un lado, la preservación de la diversidad en la población de soluciones gracias a la introducción de un enfoque multiobjetivo; y, por otro lado, un mejor aprovechamiento de la capacidad computacional a través de la paralelización de la evaluación de la función objetivo.

Las mejoras obtenidas con las nuevas propuestas han permitido que el tiempo de computación sea compatible con una gestión de las peticiones de riego a nivel diario, para el caso de estudio planteado.

Además, se ha propuesto una mejora al método de maximización del volumen entregado por gravedad para el caso particular de redes de riego en que parte de las parcelas pueden ser regadas sin necesidad de bombeo, gracias a su diferencia de cota respecto al punto de suministro. Esta mejora se ha basado en una modificación del planteamiento del problema, que se ha orientado hacia una estrategia más voraz, de modo que, en lugar de resolver un problema de optimización para un horizonte temporal extendido, se resuelven secuencialmente tantos problemas como sean necesarios, donde cada uno de ellos únicamente contempla la operación en un instante de tiempo.

Los resultados de este enfoque también han sido satisfactorios, logrando reducir significativamente el tiempo total de computación, además de mejorar sutilmente los resultados ofrecidos por el método anterior. 


\section{CAPÍtULLO 4.}

\section{OPTIMIZACIÓN ENERGÉTICA DE SISTEMAS DE TRANSPORTE DE AGUA}

\subsection{Introducción}

A diferencia de los sistemas de riego analizados en el capítulo anterior, en los que el bombeo directo a red es bastante usual, en abastecimientos de agua en cambio, es mucho más usual bombear primero el agua a un depósito de almacenamiento, desde el cual se abastece a continuación la red de distribución por gravedad.

El hecho de introducir un sistema de almacenamiento de agua intermedio cambia totalmente el planteamiento del problema de optimización energética, ya que el caudal bombeado ya no tiene porqué seguir al caudal demandado, ofreciendo una mayor libertad a la hora de consumir energía de la red eléctrica, lo que en teoría puede dar lugar a un ahorro notable en los costes energéticos, aprovechando las tarifas más baratas o las fuentes de energías renovables cuando están disponibles. A cambio, el bombeo a depósito obliga a elevar el agua hasta una cota que a veces es excesiva para muchos puntos de consumo.

Toda esta casuística se va a analizar en la presente Tesis bajo el nombre único de “optimización energética de los sistemas de transporte de agua", los cuales constituyen en la mayoría de los casos un subsistema hidráulico que puede desgajarse del sistema global de suministro, para ser estudiado de forma independiente.

Los subsistemas de transporte de agua suelen constar de bombeos a depósito en los que no existen consumos intermedios, sino que el destino de todo el caudal bombeado son los propios depósitos. Como consecuencia, el punto de funcionamiento de las bombas en 
estos subsistemas es muy estable ya que únicamente depende de la variación en el nivel de los depósitos (excepto si estos se alimentan por su parte superior, en cuyo caso el punto de funcionamiento no se vería afectado en absoluto) y del número de bombas en paralelo que estén activas, modificando así el caudal transportado por las conducciones y, consecuentemente, las pérdidas por fricción.

Por este motivo, una simplificación de las ecuaciones hidráulicas que determinan el comportamiento de estos subsistemas asumiendo puntos de funcionamiento únicos, no incurre en un error excesivo y permite una formulación del problema de optimización mediante métodos de programación lineal.

Estos métodos, ya utilizados anteriormente para la optimización energética de este tipo de sistemas, presentan principalmente dos importantes ventajas respecto a otros métodos de optimización: por un lado, siempre garantizan que alcanzan el óptimo global, mientras haya al menos una solución factible, y, por otro lado, son muy eficientes computacionalmente, siendo capaces de obtener en décimas de segundo la solución a problemas con cientos de variables.

Así, en el presente capítulo se propone una formulación para construir de manera sistemática las ecuaciones que definen el problema linealizado de optimización energética en la operación de los sistemas de transporte de agua, a partir de un modelo hidráulico completo y una serie de parámetros configurables.

\subsection{Descripción del método por programación lineal.}

En la presente sección se describe el método lineal desarrollado en el ámbito de la Tesis para minimizar el coste de la energía en subsistemas de bombeo para redes de abastecimiento de agua, en cuya regulación participan además de las bombas, también depósitos y válvulas de regulación. Aunque el comportamiento real de estos elementos sea no lineal respecto al caudal, así como las pérdidas en las tuberías de transporte, bajo determinadas hipótesis es posible formular el problema de minimizar el coste energético como un problema lineal.

El objetivo del método de optimización es definir la programación de los elementos de regulación que configuran el subsistema (bombas y válvulas) para un periodo de tiempo determinado (normalmente las próximas 24 horas), que minimiza el coste energético 
incurrido para satisfacer las demandas, las cuales se consideran conocidas, y cumplir al mismo tiempo una serie de restricciones relativas a los niveles de los depósitos que forman parte del subsistema.

La programación de los elementos de regulación consiste en determinar los instantes de marcha y paro de cada una de las bombas, o su velocidad de giro en caso de existir bombas de velocidad variable, así como el caudal de paso permitido por las válvulas en cada uno de los intervalos de tiempo, en caso de que estas intervengan también en el proceso de regulación.

\subsubsection{Definición de los subsistemas}

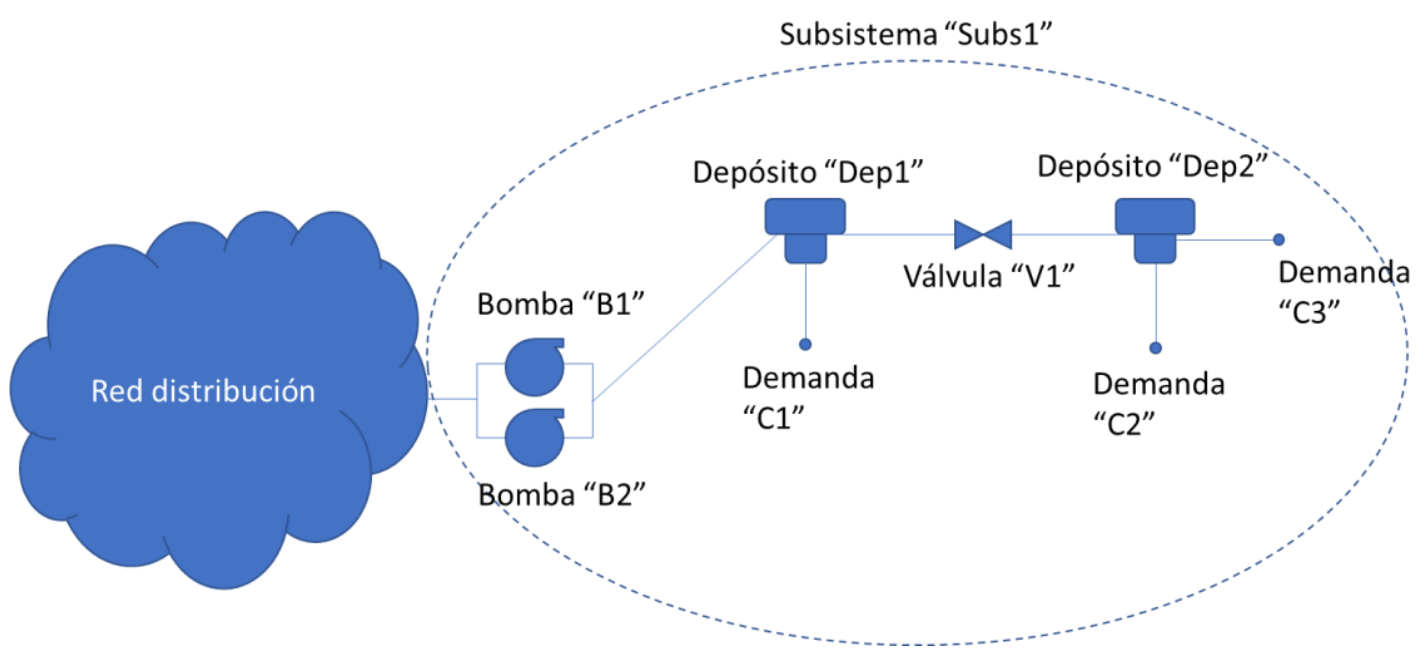

Figura 19. Esquema de los elementos que componen un subsistema de optimización dentro de una red de distribución de agua.

Un subsistema de bombeo se define como un subconjunto de elementos de una red de distribución de agua que contiene, al menos, una bomba, un depósito y un nudo de demanda, aunque usualmente las demandas serán múltiples. El subsistema debe incluir todos los elementos de regulación que afectan a las condiciones de suministro de las demandas. Si el objetivo de la optimización es reducir los costes energéticos en el subsistema, deberá haber necesariamente alguna bomba, y si se pretende controlar además el caudal transferido entre depósitos el subsistema deberá contener al menos una válvula. La presencia de al menos un depósito es requerida para independizar el caudal bombeado del consumo en cada instante, pues en otro caso la optimización se limitaría a consignar la presión de salida de las bombas para satisfacer la presión mínima. 
El proceso de optimización trabaja sobre un modelo hidráulico del sistema, definido por un fichero de EPANET tipo .INP, en el que se declaran todas las conducciones y elementos de regulación, sus propiedades físicas y curvas de comportamiento, así como los factores de demanda de cada uno de los nudos que forman parte de los diferentes subsistemas, agrupados por una misma curva de modulación. El modelo hidráulico definido en el fichero .INP permite resolver las ecuaciones de conservación de masa y de energía (no lineales) que resultan de la configuración física del sistema. Algunos de los resultados, como el caudal trasegado por las bombas, la potencia consumida por estas o los caudales entrantes/salientes de los depósitos, se utilizan para determinar los coeficientes del modelo lineal. Las variables que restan por definir hacen referencia al modo de operación del sistema, y en particular a los flujos bombeados o transferidos entre depósitos, los cuales se desea optimizar sometidos a unas restricciones.

Para declarar la configuración de los diferentes subsistemas de regulación que pueden formar parte de la red global de transporte y distribución de agua, se propone declarar todos los elementos específicos que intervienen en la regulación y sus restricciones en un archivo de texto adicional, tabulado y estructurado en distintas secciones. En concreto, el archivo deberá contener las siguientes secciones para dar cabida a todos los datos requeridos:

- Sección [SYSTEMS]: En esta sección se declaran los diferentes subsistemas que configuran la red. Se estructura en dos columnas. La primera identifica a cada subsistema mediante su ID. La segunda columna establece una restricción de simultaneidad en las bombas del subsistema limitando el número de bombas que pueden trabajar simultáneamente. Esta restricción se justifica en base a la posibilidad de que existan bombas de reserva en una estación de bombeo, o que haya alguna limitación en el caudal máximo bajo ciertas circunstancias.

- Sección [TANKS]: Contiene la declaración de los depósitos pertenecientes a cada subsistema, así como información complementaria acerca de los mismos. Se estructura en cuatro columnas. En la primera se indica el ID del subsistema al que pertenece el depósito. En la segunda se declara el ID del depósito. En la tercera columna se define el nivel mínimo permitido, y en la cuarta el nivel máximo permitido. Estos dos últimos parámetros en la práctica serán distintos a los límites físicos del depósito, siendo siempre más restrictivos que estos. 
- Sección [PUMPS]: Contiene la declaración de las bombas de cada subsistema, así como información complementaria acerca de las mismas. Se estructura en cuatro columnas. En la primera se indica el ID del subsistema al que pertenece la bomba. En la segunda se declara el ID de la bomba. En la tercera se define la velocidad (relativa) nominal de la bomba, es decir, la velocidad de giro que se va a imponer en el modelo cuando corresponda a la situación de marcha. Cabe señalar que en la metodología expuesta en este capítulo siempre se considerarán las bombas de velocidad fija, aunque esta puede ser distinta a 1. Finalmente, se indica mediante un valor booleano si el elemento se considerará como variable de decisión o no. En caso negativo, la programación inicial se tomará como condición de contorno.

- Sección [VALVES]: Contiene la declaración de las válvulas de regulación de cada subsistema, así como información complementaria acerca de las mismas. Se estructura en cuatro columnas. En la primera se indica el ID del subsistema al que pertenece la válvula. En la segunda se declara el ID de la válvula. En la tercera se define el caudal nominal de la válvula. Este caudal será inferior al máximo caudal de paso correspondiente a la posición de válvula completamente abierta bajo determinadas condiciones hidráulicas, a fin de garantizar la factibilidad de las soluciones. Finalmente, se indica mediante un valor booleano si el elemento se considerará como variable de decisión o no.

- Sección [DEMANDS]: Contiene la declaración de las demandas satisfechas por los depósitos de cada subsistema. Se estructura en dos columnas. En la primera se indica el ID del subsistema al que pertenece la demanda. En la segunda se declara el ID de la curva de modulación que agrupa a un determinado número de demandas. Un mismo subsistema puede alimentar a diversos grupos de demanda, que deben estar declarados en el modelo de la red de EPANET.

A continuación, se muestra un ejemplo del fichero de datos que identifica los elementos de regulación que forman parte de un subsistema, y que van a intervenir en el problema de optimización. El trazado completo de la red de transporte y distribución de cada subsistema se supone declarado en un fichero complementario de EPANET tipo .INP, como ya se ha comentado. 
Se admite bajo el planteamiento propuesto que la red completa puede dividirse en varios subsistemas a efectos de regulación. Cada subsistema es alimentado o controlado por una estación de bombeo, pudiendo contener además depósitos y válvulas, aunque no necesariamente.

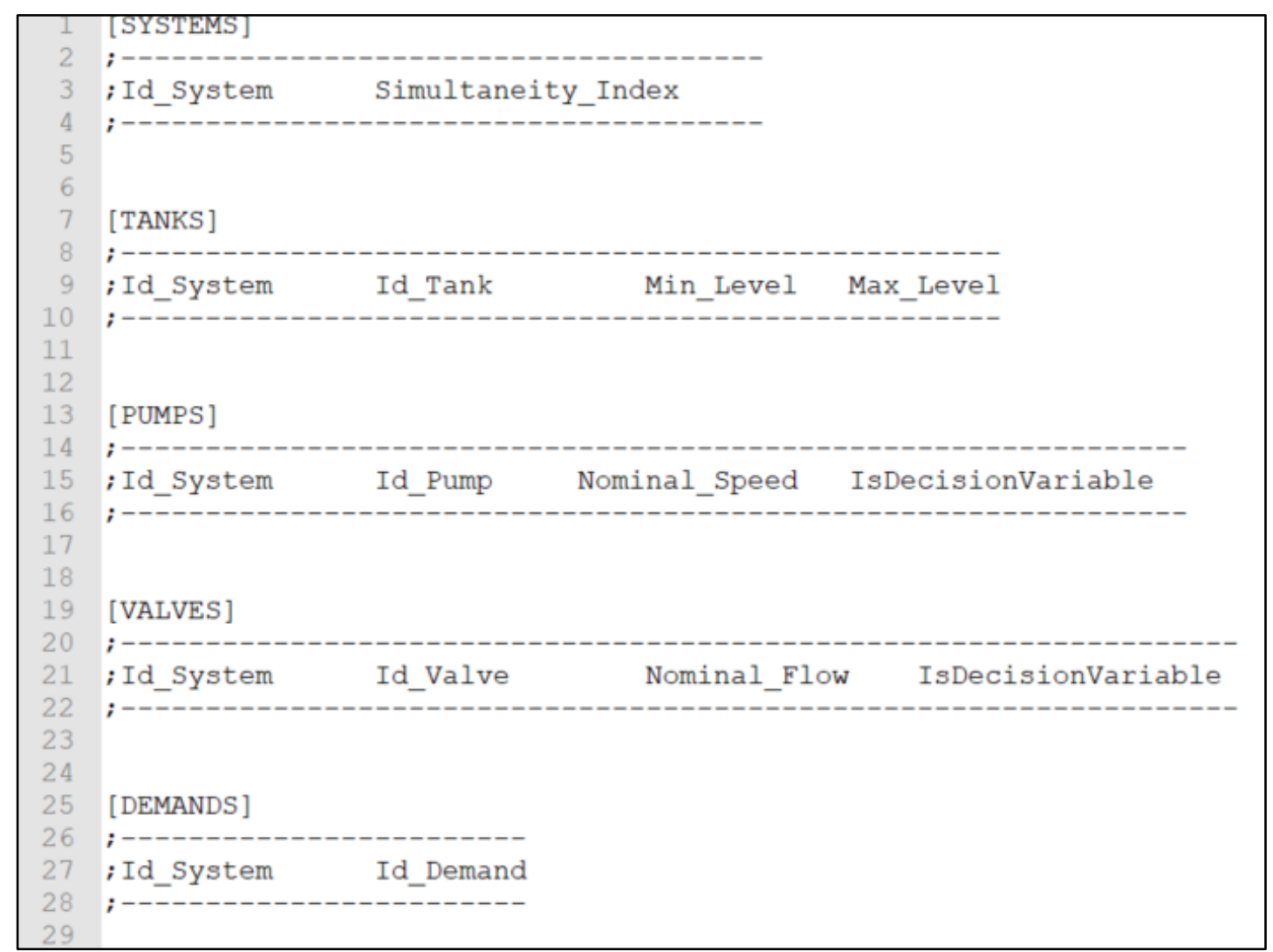

Figura 20. Estructura del archivo de configuración propuesto para la optimización mediante programación lineal.

\subsubsection{Consideraciones sobre la optimización lineal}

Tal como se ha expuesto con anterioridad, los métodos de programación lineal tienen principalmente dos ventajas: el cálculo es muy eficiente computacionalmente, con lo que se puede obtener la solución en muy poco tiempo (décimas de segundo para el caso de estudio planteado en este capítulo); además, si existe factibilidad, siempre garantizan que la solución alcanzada responda al óptimo global del problema.

El gran inconveniente de estos métodos es que el problema se ha de plantear como un conjunto de expresiones lineales, tanto para declarar la función objetivo como las restricciones, y dado que las ecuaciones de la hidráulica son no lineales, se deben realizar una serie de aproximaciones que conllevarán un error más o menos importante dependiendo de las características del sistema. Este error de linealización en la práctica va a suponer que 
la solución óptima devuelta por el algoritmo (programación de los elementos de regulación), cuando se reproduzca en el modelo hidráulico de EPANET no va a proporcionar las mismas trayectorias para los niveles de los depósitos, ni el mismo consumo energético previsto por el modelo lineal. Por ello, puede que la solución se aleje en cierto grado del óptimo verdadero.

Aun así, puesto que en un diseño racional de los sistemas de transporte y distribución las pérdidas en las conducciones deben ser pequeñas respecto a la energía estrictamente necesaria para elevar el agua o para comunicar la presión requerida a los usuarios, esta metodología puede ser de aplicación en numerosos casos de bombeos a depósito, siempre que el caudal trasegado por las bombas se vea poco afectado por las condiciones de trabajo de los elementos disipativos de energía.

\subsubsection{Planteamiento del problema de optimización lineal}

Se define un horizonte temporal y una discretización del periodo de optimización en un número de intervalos de tiempo, dentro de los cuales se supone que las condiciones hidráulicas no varían. Típicamente, el horizonte temporal será de 24 horas, debido al carácter cíclico de las demandas urbanas y de las tarifas eléctricas, en cuyo comportamiento se distingue claramente un patrón que se repite diariamente. La discretización más común es la horaria, ya que puede admitirse que los consumos varían poco en ese tiempo pudiendo reproducir bien la variación de la demanda diaria con dicha resolución, mientras que en el mercado eléctrico los distintos precios de la energía se establecen también hora a hora.

Las variables de decisión del sistema se definen como variables continuas $x_{i, t} \in$ $[0,1]$, donde $i$ es el elemento de regulación sobre el que se actúa (bomba o válvula), y $t$ es el intervalo de tiempo. Si el problema se ha discretizado en $N_{t}$ intervalos de tiempo, y el subsistema contiene $N_{e}$ elementos de regulación, el número de variables será $N_{v a r}=N_{t}$. $N_{e}$.

La interpretación de las variables es ligeramente distinta para bombas y válvulas. En ambos casos un 0 indica que a lo largo del instante de tiempo considerado el elemento no trasiega ningún caudal, es decir, una bomba estaría parada y una válvula estría cerrada. Análogamente, un 1 indica que la bomba o la válvula trasiegan su caudal nominal $Q_{i}$ a lo largo del intervalo de tiempo correspondiente. Sin embargo, si el resultado es un valor entre 0 y 1 se debe interpretar, en el caso de una válvula, que su caudal sigue siendo constante a 
lo largo del intervalo pero inferior al caudal nominal, reduciéndose en proporción al valor de la variable. En el caso de una bomba, se entenderá que es el tiempo de marcha lo que se reduce proporcionalmente al valor de la variable. Es decir, si, por ejemplo, $\mathrm{x}=0.5$, la bomba estaría funcionando a su velocidad nominal durante media hora y el resto del intervalo estaría parada. Alternativamente, en caso de que la bomba dispusiera de variador de velocidad, se podría calcular la velocidad de giro que debería mantenerse durante todo el intervalo para conseguir que el caudal entregado se corresponda con la fracción deseada del caudal nominal, mediante la aplicación de las leyes de semejanza.

En cuanto a la potencia consumida por las bombas se supondrá que esta es proporcional al caudal impulsado, lo que conlleva admitir que la altura de elevación y el rendimiento se mantienen constantes para cualquier punto de trabajo. $\mathrm{O}$ bien que estos se mantienen constantes durante la fracción de tiempo que dura el bombeo y que dicho punto es siempre el mismo cuando la bomba está en marcha.

\subsubsection{Función objetivo}

Si el objetivo de la optimización es minimizar el coste energético de bombeo, la función objetivo se define para el modelo lineal como:

$$
\min f(x)=\sum_{t=1}^{N t}\left(\sum_{i=1}^{N e} P_{i} \cdot \Delta T \cdot C e_{t} \cdot x_{i, t}\right)
$$

donde:

$N_{t}=$ número de intervalos de tiempo considerados en el periodo de optimización.

$N_{e}=$ número de elementos de regulación que intervienen en el problema.

$P_{i}=$ potencia consumida por el elemento $i$ a velocidad nominal $(\mathrm{kW})$. Naturalmente, los términos $P_{i}$ correspondientes a las válvulas del sistema tendrán un valor 0 , y solamente intervendrán las variables correspondientes a las bombas, pero se ha preferido plantearlo de manera general por sencillez.

$\Delta T=$ duración de cada intervalo (h).

$C e_{t}=$ coste de la energía en el instante $t(€ / \mathrm{kWh})$.

$x_{i, t}=$ fracción de tiempo o de caudal correspondiente al elemento $i$ en el instante $t$ (adimensional, entre 0 y 1 ) 


\subsubsection{Funciones de transferencias de flujo}

Cuando se activa/desactiva una bomba o se regula una válvula, además de modificar el caudal de paso a través del mismo, cambia también el caudal entrante a los depósitos. Además, el caudal saliente de los mismos está afectado por el factor de consumo del subsistema alimentado, aunque no necesariamente al $100 \%$. Es decir, el caudal bombeado o regulado puede afectar al caudal entrante a uno o más depósitos, y estos a su vez pueden alimentar a uno o más subsistemas. La relación existente entre unos caudales y otros viene determinada por el modelo hidráulico, y en particular por las ecuaciones de balance de masa y de energía establecidas entre todos los nudos y elementos de paso respectivamente.

Concretamente, el comportamiento hidráulico debe cumplir con las ecuaciones de conservación de masa, formuladas para todos los nudos del subsistema:

$$
\sum Q_{j}=0
$$

donde el sumatorio se extiende a todas las tuberías incidentes en cada nudo más las demandas aplicadas.

Y también se deben cumplir las ecuaciones de comportamiento hidráulico de los elementos lineales, tanto tuberías, como bombas y válvulas:

$$
H_{i}-H_{j}=f\left(Q_{i j}\right)
$$

que indican que la diferencia en la altura piezométrica entre dos extremos de cada línea es una función del caudal que trasiega dicha línea. La función $f$, en general, es no lineal. En consecuencia, para conseguir que el método de optimización propuesto sea de aplicación, se debe realizar una linealización de este último conjunto de ecuaciones, pudiéndose expresar como sigue:

$$
H_{i}-H_{j}=a+b \cdot Q_{i j}
$$

En este punto, se debería obtener para cada línea el valor de los coeficientes $a$ y $b$ mediante la definición de unos criterios sistemáticos.Una alternativa a formular explícitamente estas ecuaciones, de las cuales las correspondientes al balance de energías serán en general no lineales, consiste en declarar unas funciones de transferencia de flujo $\varphi_{i, d}$ que relacionen el caudal de paso por cada elemento de regulación $i$ con el caudal entrante o saliente de cada depósito $d$, y otras funciones similares $\varphi_{c, d}^{\prime}$ que relacionen las demandas en cada subsistema alimentado $c$ con el caudal entrante/saliente de cada depósito $d$. En la 
hipótesis de linealización se asumirá que dichos coeficientes son constantes para cualquier intervalo de tiempo, y su determinación se realizará con la ayuda del modelo, tal como se describe más adelante en el apartado 4.2.4.

\subsubsection{Restricciones}

El problema planteado conlleva la necesidad de definir una serie de restricciones para mantener los niveles de los depósitos dentro de los límites permitidos y, además, establecer el nivel de estos al final del periodo de optimización. De no imponer esta última restricción la solución óptima tendería siempre a vaciar los depósitos, puesto que el agua no bombeada no incurre en ningún coste.

Una estrategia usual es imponer la restricción de que los depósitos estén al final del periodo de optimización al mismo nivel que al inicio del mismo. En el apartado 4.3 se justifica esta elección. Para evitar la repetición estricta de los ciclos diarios, se puede extender el periodo de optimización a una semana y así aprovechar las tarifas especiales de fin de semana, siempre que el volumen del depósito lo permita. Otras veces, cuando el volumen del depósito es pequeño y se realizan varios ciclos diarios, en el proceso de optimización primará más el hecho de mantener el nivel del depósito dentro de los márgenes establecidos, que intentar aprovechar las tarifas más baratas para llenar el mismo, habiendo poco margen para el ahorro energético.

Así pues, para garantizar que el nivel de los depósitos se mantenga entre un umbral máximo y uno mínimo, se propone incorporar dos restricciones para cada intervalo de tiempo $t_{k} \in\left[1, N_{t}-1\right]$ y por cada depósito $d$, las cuales se definen como sigue:

$$
\begin{aligned}
& H_{\text {ini,d }}+\frac{1}{S_{d}} \sum_{t=1}^{t_{k}}\left(\sum_{i=1}^{N e}\left(\varphi_{i, d} \cdot Q_{i} \cdot \Delta T \cdot x_{i, t}\right)+\sum_{c=1}^{N c}\left(\varphi_{c, d}^{\prime} \cdot Q_{c, t} \cdot \Delta T\right)\right) \leq H_{\text {max }, d} \\
& H_{\text {ini,d }}+\frac{1}{S_{d}} \sum_{t=1}^{t_{k}}\left(\sum_{i=1}^{N e}\left(\varphi_{i, d} \cdot Q_{i} \cdot \Delta T \cdot x_{i, t}\right)+\sum_{c=1}^{N c}\left(\varphi_{c, d}^{\prime} \cdot Q_{c, t} \cdot \Delta T\right)\right) \geq H_{\text {min }, d}
\end{aligned}
$$

donde:

$$
\begin{aligned}
N_{e}= & \text { número de elementos de regulación que intervienen en el problema (bombas y } \\
& \text { válvulas) }
\end{aligned}
$$


$N_{c}=$ número de grupos de demandas con curva de modulación común pertenecientes al subsistema.

$H_{\text {ini,d }}=$ nivel inicial del depósito $d(\mathrm{~m})$.

$H_{\text {min,d }}=$ nivel mínimo permitido del depósito $d(\mathrm{~m})$.

$H_{\text {max }, d}=$ nivel máximo permitido del depósito $d(\mathrm{~m})$.

$S_{d}=$ superficie del depósito $d\left(\mathrm{~m}^{2}\right)$.

$t_{k}=$ intervalo de optimización para el que se define la restricción.

$Q_{i}=$ caudal nominal del elemento de regulación $i\left(\mathrm{~m}^{3} / \mathrm{h}\right)$.

$Q_{c, t}=$ caudal total del grupo de demandas $c$ que comparten la misma curva de modulación en el instante $t\left(\mathrm{~m}^{3} / \mathrm{h}\right)$.

$\varphi_{i, d}=$ coeficiente que relaciona el caudal entrante/saliente del depósito $d$ con el caudal de paso por el elemento de regulación $i$

$$
\varphi_{i, d}=\frac{Q_{i, d}}{Q_{i}}
$$

$\varphi_{c, d}^{\prime}=$ coeficiente que relaciona el caudal entrante/saliente al depósito $d$ con el caudal de demanda $c$.

$$
\varphi_{c, d}^{\prime}=\frac{Q_{c, d}}{Q_{c}}
$$

Finalmente, con el objetivo de garantizar que al final de la simulación se alcanza al menos el nivel inicial del que parte cada depósito, para el último intervalo de tiempo se impondrá una restricción adicional por cada depósito:

$$
\sum_{t=1}^{N t}\left(\sum_{i=1}^{N e}\left(\varphi_{i, d} \cdot Q_{i} \cdot \Delta T \cdot x_{i, t}\right)+\sum_{c=1}^{N c}\left(\varphi_{c, d}^{\prime} \cdot Q_{c, t} \cdot \Delta T\right)\right) \geq 0
$$


Nótese que, tanto la función objetivo como las restricciones de nivel son lineales, debido al hecho de considerar que los caudales nominales de las válvulas y las bombas, así como la potencia consumida por estas son valores prefijados de antemano, y las funciones de transferencia $\varphi$ se consideran constantes.

\subsubsection{Cálculo de las funciones de transferencia}

Para la obtención de las funciones de transferencia se puede utilizar un modelo hidráulico en EPANET del sistema global, previamente cargado para el periodo de optimización, y realizar una serie de simulaciones. Para ello deben seguirse los siguientes pasos:

- Eliminar del modelo todos los controles que hagan referencia a los elementos de regulación, tanto los controles simples como las reglas.

- Consultar sobre el modelo los datos relativos a los depósitos:

○ Diámetro, para el cálculo de la superficie $S_{d}=\frac{\pi \phi^{2}}{4}$.

○ Nivel inicial $H_{\text {ini,d }}$.

- Con todos los elementos de regulación cerrados, consultar el caudal de entrada o salida a los depósitos.

- Abrir uno a uno los elementos de regulación a su caudal o velocidad nominal, y consultar el nuevo caudal de entrada o salida de los depósitos.

- Consultar el caudal de paso por cada elemento de regulación $Q_{i}$ y la potencia consumida, en el caso de las bombas, $P_{i}$.

- Calcular las funciones de transferencia que relacionan la variación del caudal de entrada/salida a los depósitos en función del caudal de paso por los elementos de regulación $\varphi_{i, d}=\frac{Q_{i, d}}{Q_{i}}$. Aquí, $Q_{i, d}$ representa la fracción del caudal trasegado por el elemento $i$ que llega al depósito $d$, y se obtiene como la diferencia entre el caudal de entrada al depósito una vez se ha abierto el elemento $i$, y el caudal de entrada al depósito con todos los elementos de regulación cerrados.

- Obtener las demandas totales en cada instante $Q_{c, t}$ multiplicando la curva de modulación por la suma de las demandas base de todos los nudos del modelo que comparten dicha curva de modulación. 
- Obtener el caudal entrante o saliente de cada depósito en el primer instante de simulación.

- Anular el primer coeficiente de la curva de modulación de cada demanda y volver a obtener el caudal en los depósitos.

- Calcular las funciones de transferencia de las demandas $\varphi_{c, d}^{\prime}=\frac{Q_{c, d}}{Q_{c}}$. Aquí, $Q_{c, d}$ representa la fracción del caudal demandado por el conjunto de nudos $c$ que sale del depósito $d$, y se obtiene como la diferencia entre el caudal de salida del depósito en el primer instante, y el caudal de salida del depósito cuando se ha anulado el primer coeficiente de la curva de modulación de la demanda $c$. Por su parte, $Q_{c}$ debe corresponder al caudal demandado por $c$ en el primer instante de simulación.

\subsubsection{Obtención del precio de la energía}

En la página web del operador de mercado eléctrico designado para la gestión del mercado diario e intradiario de electricidad en la Península Ibérica (OMIE), se publican todos los días del año los resultados de la sesión de mercado que se lleva a cabo a las 12:00 CET en la que se fijan los precios de la electricidad en toda Europa para las veinticuatro horas del día siguiente.

Los datos se pueden descargar en archivos de texto plano mediante la siguiente URL: https://www.omie.es/es/file-download?parents\%5B0\%5D=marginalpdbc\&filename=marginalpdbc $\{$ fecha $\} .1$ sustituyendo el parámetro \{fecha\} por la fecha deseada en formato "yyyyMMdd". Por ejemplo, los precios de la electricidad para el 14/01/2021 se obtendrían directamente desde: https://www.omie.es/es/file-download?parents\%5B0\%5D=marginalpdbc\&filename=marginalpdbc 20210114.1

El archivo descargado es de tipo CSV, utilizando el carácter ';' como separador. Contiene una fila de cabecera con el texto "MARGINALPDBC", indicando que lo que contiene el documento es el precio marginal de la electricidad (precio de la última oferta de venta que ha sido necesario asignar para cubrir la demanda en una convocatoria de mercado) en el programa diario base de casación (PDBC). 


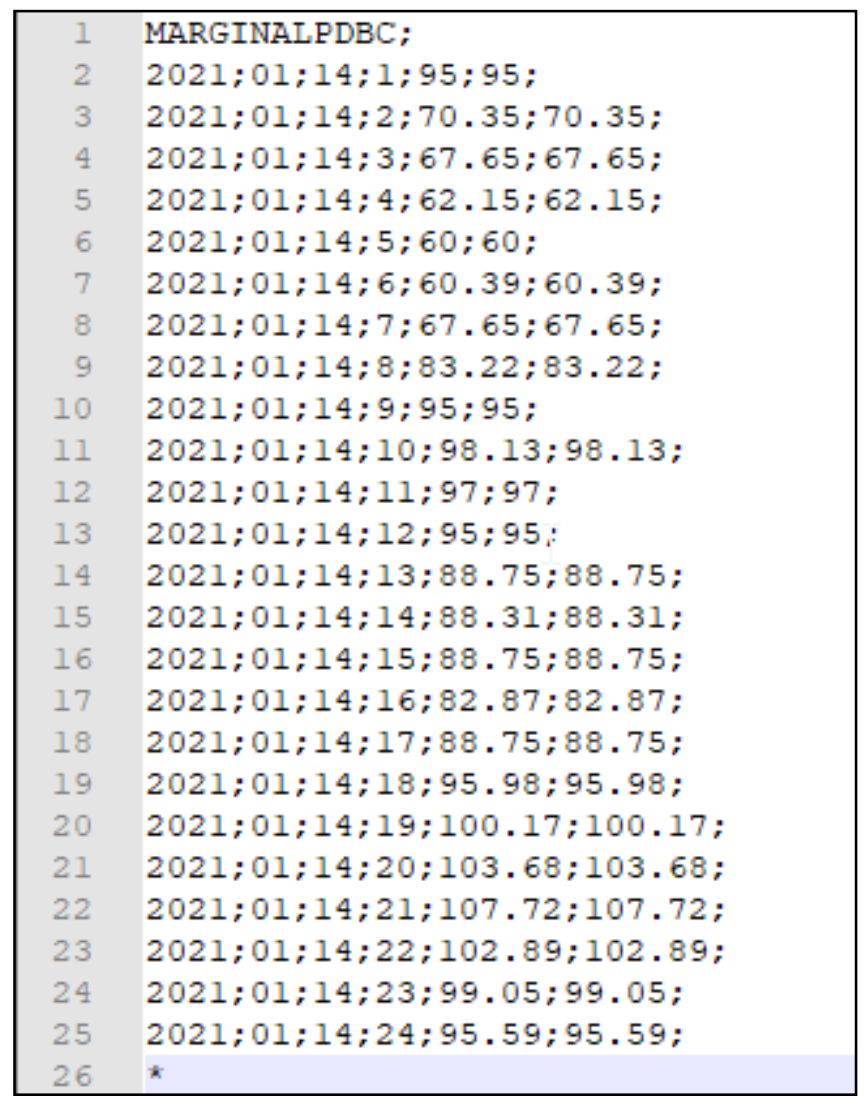

Figura 21. Ejemplo de archivo descargado de OMIE que contiene los precios de la energía hora a hora para el día $14 / 01 / 2021$

El contenido se divide en las siguientes seis columnas:

- Año

- Mes

- Día

- Periodo horario (1 indica el periodo entre las 00:00 y la 01:00)

- Precio final de la energía para Portugal (€/MWh)

- Precio final de la energía para España (€/MWh)

Hay que tener en cuenta que los datos están disponibles a partir de las 12:00 del mediodía, con lo que si se realiza una optimización a futuro para un horizonte de 24 horas antes de las 12:00 no se dispondrá todavía de todos los precios de la energía. 


\subsubsection{Comparación de resultados}

Los resultados del problema de optimización se concretan en un conjunto de reglas a aplicar en cada instante sobre cada elemento de regulación de acuerdo con la sintaxis:

\section{LINK IDlinea setting AT TIME tiempo}

conforme a la sintaxis empleada por EPANET para las reglas simples, donde IDlínea es el identificador del elemento de regulación (bomba o válvula), setting es la consigna de caudal/estado para las válvulas, o la velocidad de giro/estado para las bombas, y tiempo es el instante correspondiente, expresado en horas desde el inicio de la simulación.

Una vez resuelto el problema de optimización, se añaden los nuevos controles obtenidos al modelo ya cargado al que, previamente, se habían eliminado los controles correspondientes a los elementos de regulación del subsistema. De este modo se dispone de dos modelos para el mismo periodo de tiempo, el original con las reglas que se llevarían a cabo si no se aplica la optimización, y el nuevo modelo optimizado.

Se simulan ambos modelos y se calcula el coste total de la energía consumida en cada caso. Para el modelo lineal, el cálculo de la energía consumida es inmediato a partir de la Ecuación (32). Sin embargo, para el modelo no lineal el cálculo debe llevarse a cabo mediante la Toolkit de EPANET, a través de la cual se puede consultar instante a instante la potencia consumida por cada bomba, la cual habrá que multiplicar por la duración hasta el siguiente instante de cálculo hidráulico y por el coste de la energía en dicho periodo.

$$
C t=\sum_{t=1}^{N t}\left(\sum_{i=1}^{N e} P_{i}(t) \cdot \Delta T(t) \cdot C e_{t}\right)
$$

Nótese la diferencia con la función objetivo del problema lineal: en este caso, la potencia consumida por cada bomba dependerá del estado hidráulico de la red siguiendo las leyes no lineales de la hidráulica. Además, los intervalos de tiempo ya no tienen por qué ser constantes, dependerá de los eventos que se desencadenen (controles, reglas, llenado o vaciado de depósitos). Por otra parte, puesto que EPANET calcula la potencia consumida a partir de la altura, caudal y rendimiento de las bombas en cada instante, siendo este variable con el caudal, conviene que la potencia asumida en el modelo lineal esté acorde con los valores medios de la potencia real consumida para el rango de caudales esperado. 
Estas diferencias causarán una discrepancia entre los resultados del algoritmo lineal de optimización y los calculados con el modelo matemático no lineal. Tal como se ha comentado anteriormente, esta discrepancia puede ser mayor o menor en función de las características físicas del subsistema y, en consecuencia, el resultado puede alejarse del óptimo global o incluso también podría llegar a ser no factible por rebasar los límites de los depósitos. Por este motivo, la metodología de optimización por programación lineal exige un estudio previo de aplicabilidad en los subsistemas a tratar.

\subsection{Criterio para el establecimiento del valor final del nivel de los depósitos}

En el proceso de optimización en tiempo real de la programación del bombeo en redes hidráulicas, se debería abarcar un horizonte temporal para el cual una variación en el valor final de las variables de estado (niveles de los depósitos) no afecte al resultado. Pero esto puede suponer la necesidad de periodos largos que ralenticen o, incluso, imposibiliten el proceso de optimización.

Típicamente se establecen horizontes de 24 horas debido al carácter cíclico de las demandas y de las tarifas eléctricas. Pero este periodo no es lo suficientemente extenso como para que el valor final no influya en el resultado. Por ello, la elección de las condiciones de contorno es determinante. A continuación, se estudia la opción de restringir en cada proceso de optimización la libertad en el nivel final de cada depósito, de modo que se alcance siempre, al menos, el mismo valor que al inicio de la simulación.

El proceso de optimización en tiempo real consiste en generar periódicamente la programación de los elementos de regulación (bombas y válvulas) desde el instante actual hasta el mismo instante del día siguiente. La frecuencia de generación de las programaciones debe garantizar que el nivel de los depósitos sigue la trayectoria óptima, así como también que la programación se adapte a los errores en la previsión de la demanda o a las inexactitudes del modelo hidráulico.

Póngase como caso de ejemplo un sistema sencillo con un bombeo directo a depósito y una demanda concentrada en un nudo aguas abajo del depósito. 


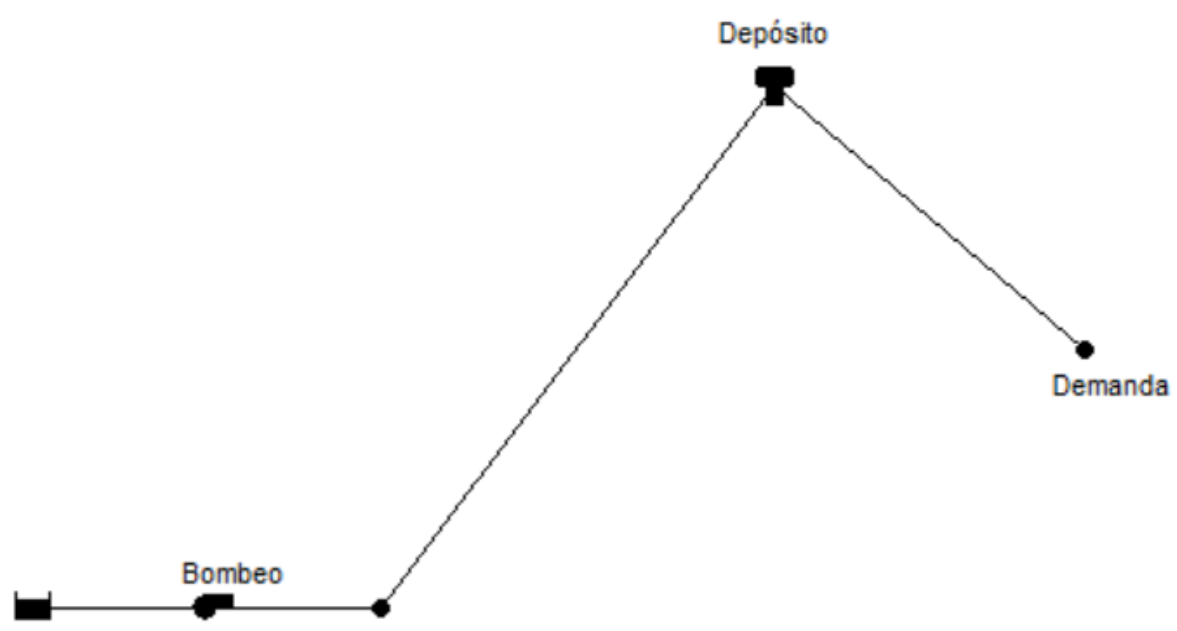

Figura 22. Esquema hidráulico del ejemplo utilizado para la validación del criterio de establecimiento del valor final del depósito.

Intuitivamente se puede deducir que el nivel de los depósitos debe ser máximo al finalizar el periodo en que la electricidad es más barata. Consecuentemente, la trayectoria óptima debe reflejar este máximo.

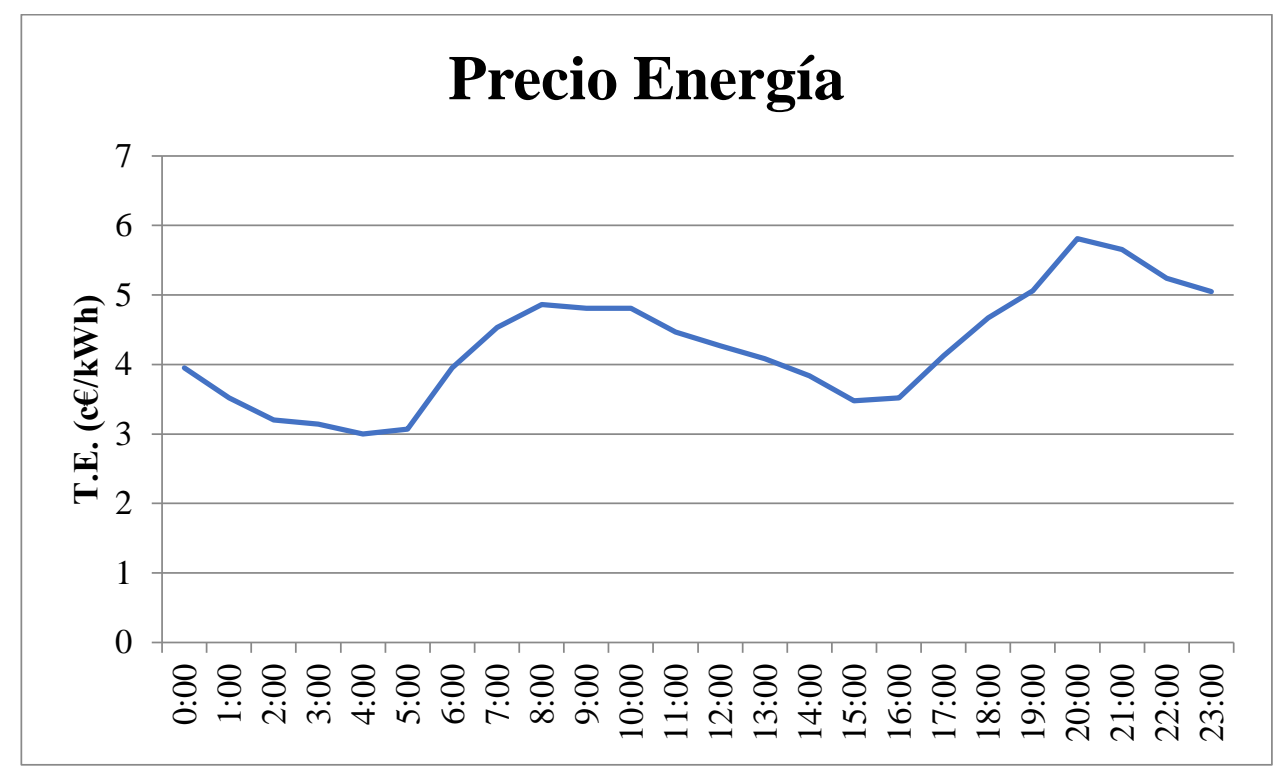

Figura 23. Precio de la energía del mercado español del día 24/03/2015. Fuente: www.omie.es

Tomando el precio de la electricidad mostrado en la Figura 23 cabría esperar que la programación óptima generara un máximo en el nivel del depósito cerca de las 6:00 y otro cerca de las 17:00. Pero si el nivel inicial del depósito no es el correspondiente a la trayectoria óptima, por el hecho de forzar a que el nivel final sea igual que el inicial, se podría caer en resultados subóptimos. Esto ocurriría si la frecuencia con la que se calculan las nuevas programaciones coincide con el horizonte. 
Para evitar el estancamiento en trayectorias subóptimas sería suficiente con calcular una nueva programación a intervalos de 1 hora tomando como nivel inicial el resultante de la optimización anterior.

Esta hipótesis se ha comprobado mediante un ejemplo, en el que se parte de depósito vacío a las 6:00 (nivel mínimo $=1 \mathrm{~m}$ ). Se han generado mediante un algoritmo de programación lineal 24 soluciones a lo largo de cada hora sucesiva del día, tomando en cada caso el nivel inicial proporcionado por la programación anteriormente calculada.

Tal como se esperaba, el nivel óptimo a las 6:00 va aumentando a medida que se avanza en el tiempo, y llega un punto a partir del cual la trayectoria que sigue el nivel del depósito se superpone en cada iteración. Suponiendo que la demanda y el precio de la electricidad puedan considerarse cíclicos, se puede concluir que la estrategia conduciría a los niveles óptimos, independientemente del nivel de partida.

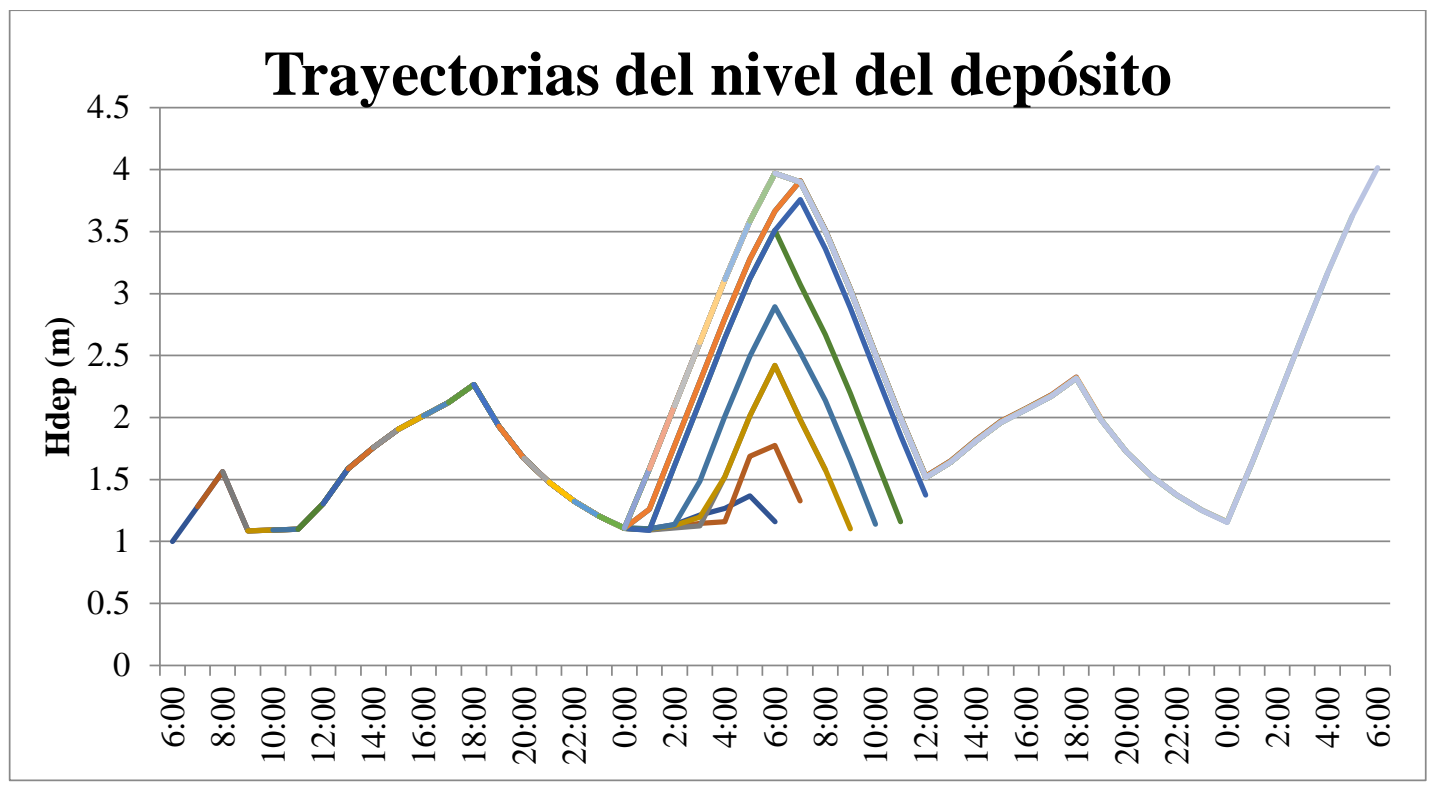

Figura 24. Niveles del depósito para cada iteración de la optimización. 
Tabla 8. Niveles del depósito

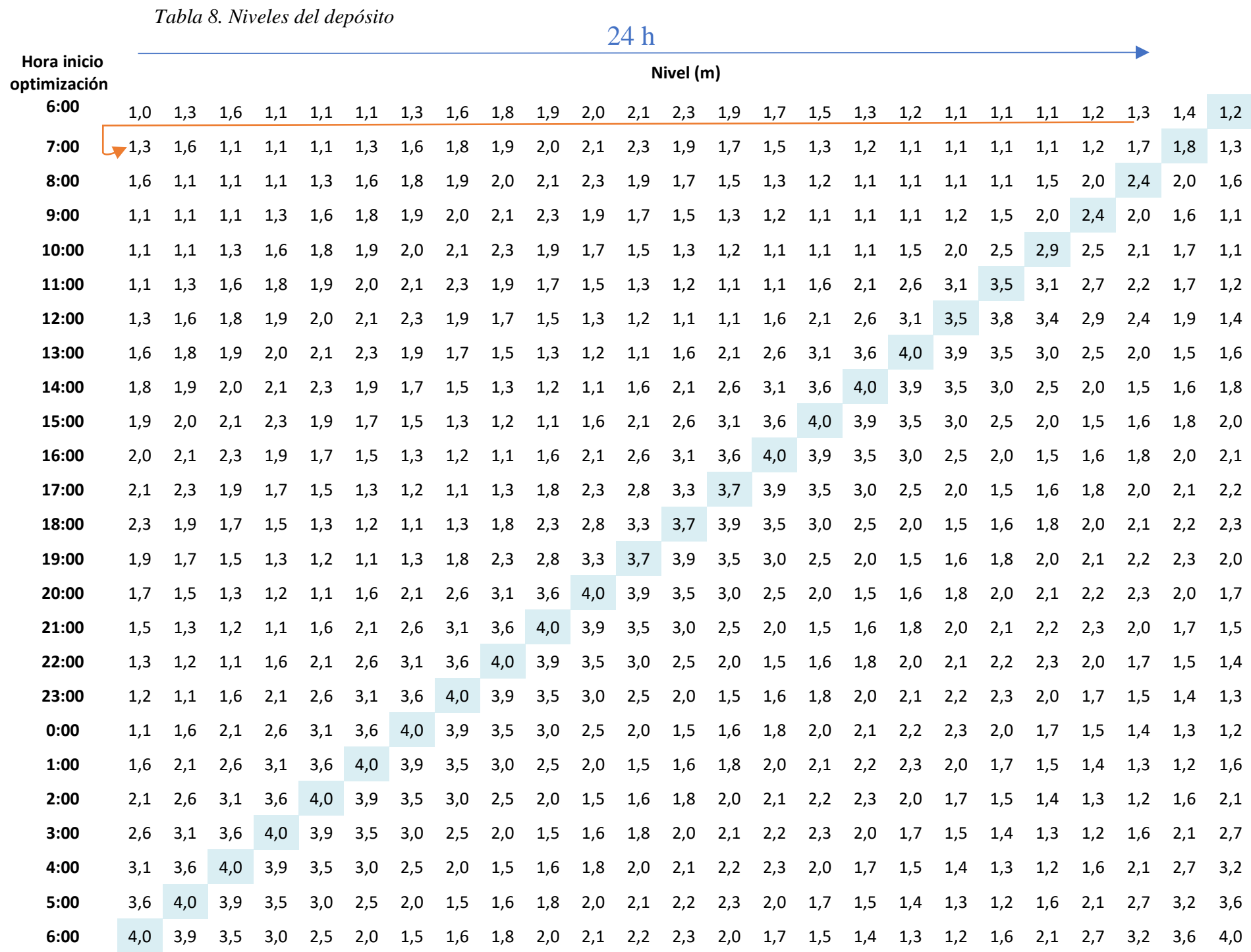

En la aplicación a casos reales se tendrá una tarifa eléctrica variable, la cual presentará diferencias significativas entre los días laborables y festivos, por ejemplo. Con el objetivo de comprobar si la estrategia presentada en este apartado conduce a trayectorias óptimas del nivel del depósito cuando no se tiene una tarifa cíclica, se ha realizado el experimento que se explica a continuación.

En primer lugar, se ha tomado una hipotética tarifa eléctrica para dos días consecutivos en la que las horas valle de un día corresponden a las horas pico del siguiente y viceversa. Con esto se trata de forzar que la trayectoria del depósito sea completamente distinta en ambos días, y se pretende comprobar si la estrategia de recalcular la optimización cada hora es eficaz para obtener dicha trayectoria. La Figura 25 muestra la tarifa utilizada. 


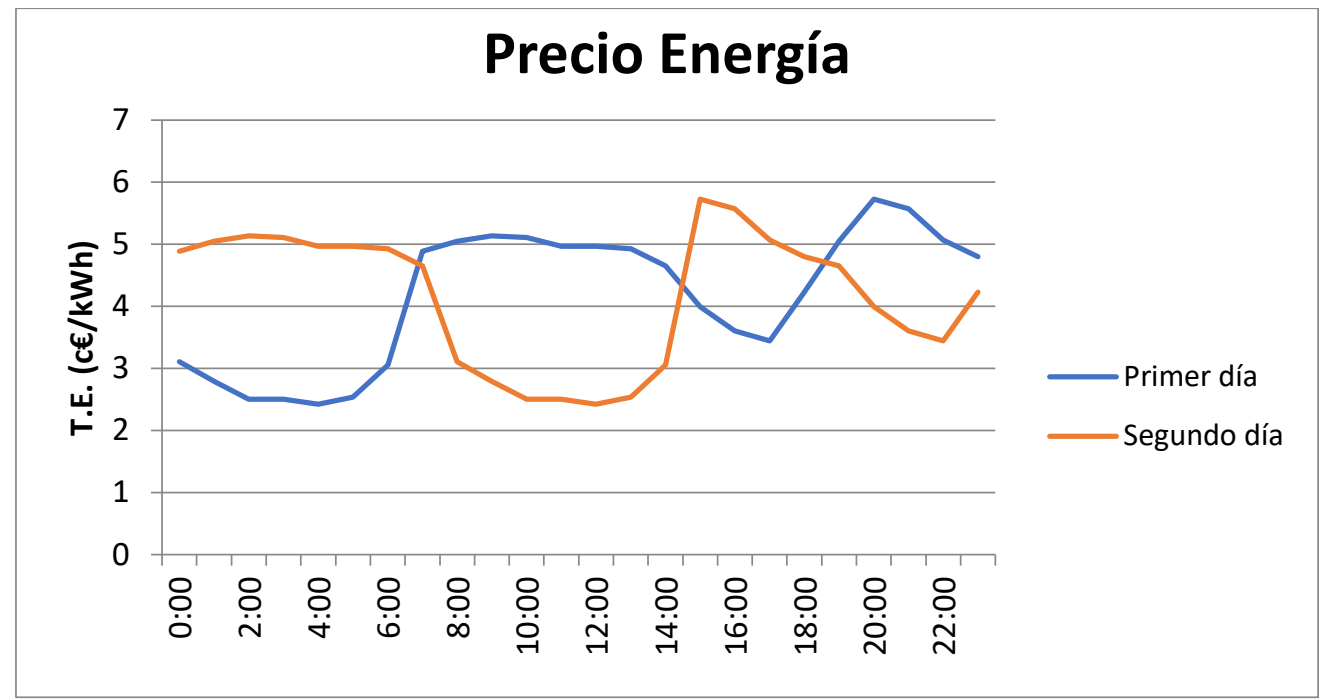

Figura 25. Precio hipotético de la electricidad para dos días consecutivos

El proceso inicial ha sido idéntico al propuesto en el ejemplo anterior, es decir, se ha calculado cada hora una optimización a 24 horas tomando en cada caso el valor inicial del depósito calculado en la iteración anterior. En la Figura 26 se incluyen únicamente la primera iteración y la última (para facilitar la lectura).

El siguiente paso ha sido realizar una optimización con un periodo de 48 horas, en la que se obtendrá la trayectoria óptima conjunta, tanto del primer día como del segundo, estableciendo el mismo nivel inicial y, como nivel final, el obtenido anteriormente. Efectivamente, se comprueba que las trayectorias de nivel del depósito obtenidas siguiendo la estrategia presente coinciden con la trayectoria obtenida estableciendo un horizonte de 48 horas.

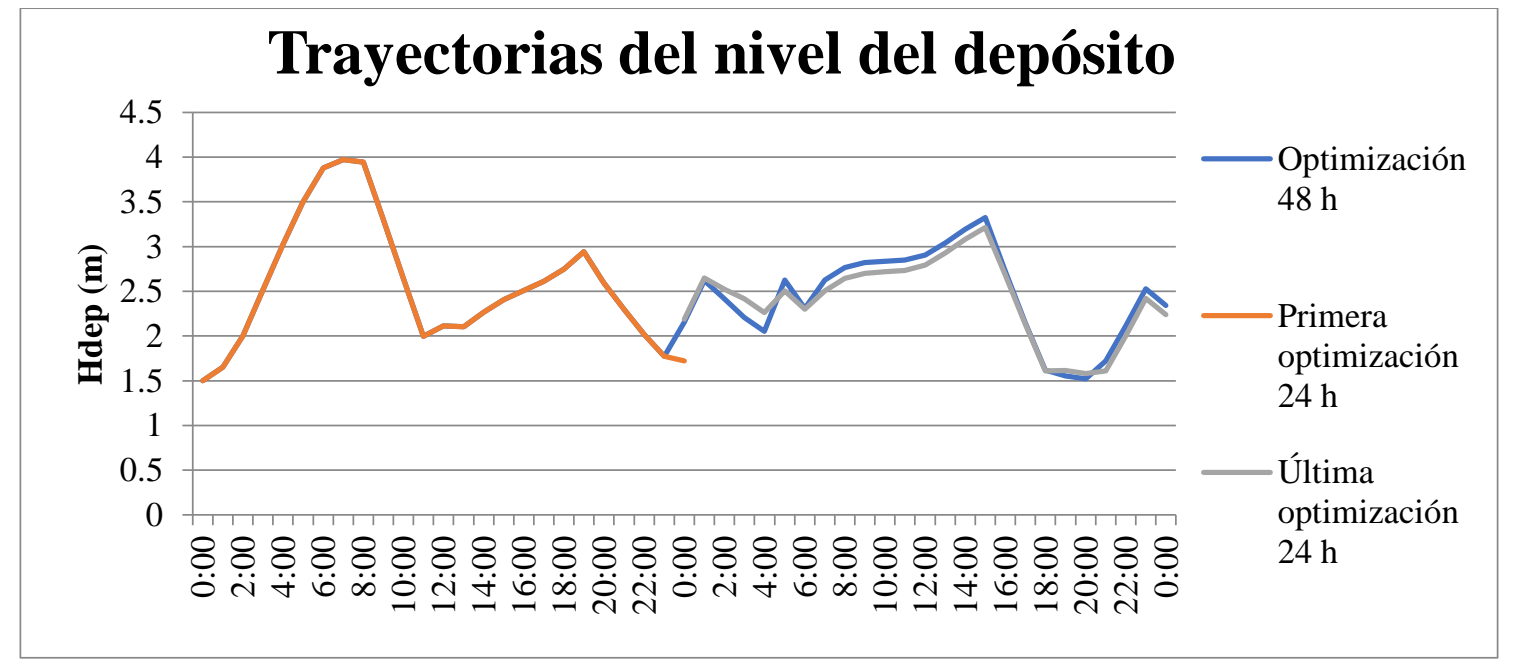

Figura 26. Trayectorias del depósito en el ejemplo propuesto 


\subsection{Caso de estudio}

El subsistema al que se ha aplicado la metodología de optimización es una infraestructura real, aunque no se identifica en la Tesis por cuestiones de confidencialidad. El subsistema toma agua de un depósito de $6.000 \mathrm{~m}^{3}$ de capacidad (dep1) y la trayectoria del nivel en el depósito se considera conocida, la cual formará parte de las condiciones de contorno del problema.

El subsistema cuenta con una estación de bombeo (Figura 27) con dos bombas idénticas en paralelo. La estación de bombeo se ubica a la salida del depósito de $6.000 \mathrm{~m}^{3} \mathrm{e}$ impulsa el agua hasta otro depósito con una capacidad de $2.500 \mathrm{~m}^{3}$ (dep2). Este, a su vez, abastece a tres sectores de demanda (dem1, dem2 y dem3) y a otro depósito de $2.500 \mathrm{~m}^{3}$ de capacidad (dep3). Entre dep2 y dep3 existe una válvula de regulación que controla el caudal de trasiego entre ambos. El dep3 abastece a un sector de demanda (dem4). En la Figura 28 se muestra un esquema del subsistema.

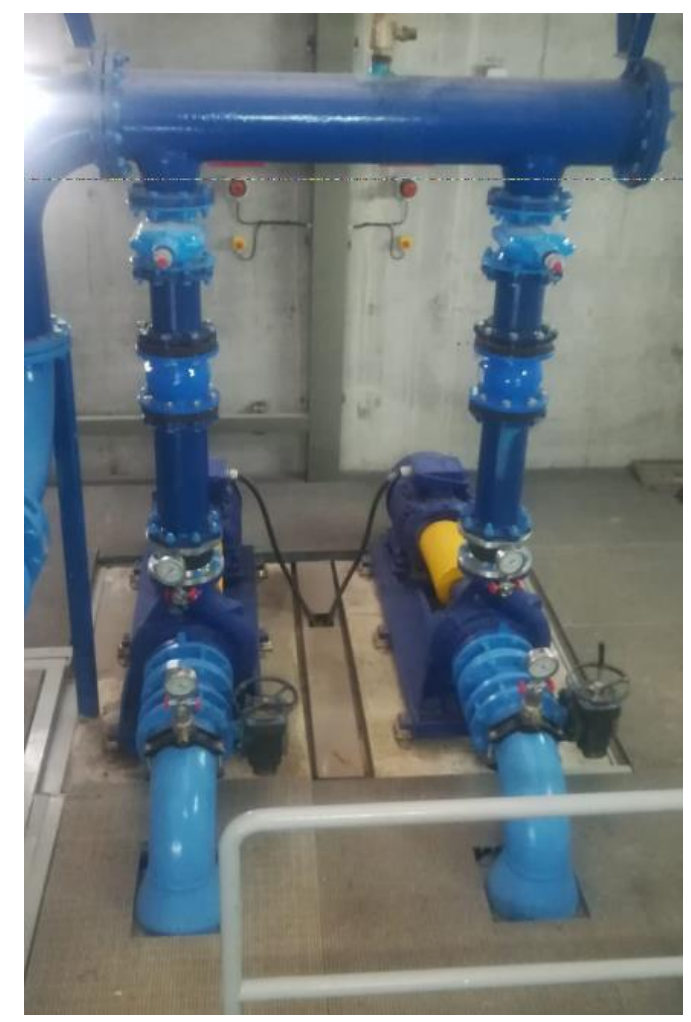

Figura 27. Estación de bombeo del subsistema a optimizar.

La cota de solera del dep1 es 112,66 m.s.n.m. y la del dep2 es 126 m.s.n.m. con lo que el desnivel que debe vencer la estación de bombeo es de unos 14 m, dependiendo de los 
niveles en cada instante. La cota de solera del dep3 es de 123,5 m.s.n.m., por lo tanto, se dispone de unos 2,5 m que permiten el trasiego entre el dep2 y el dep3 por gravedad.

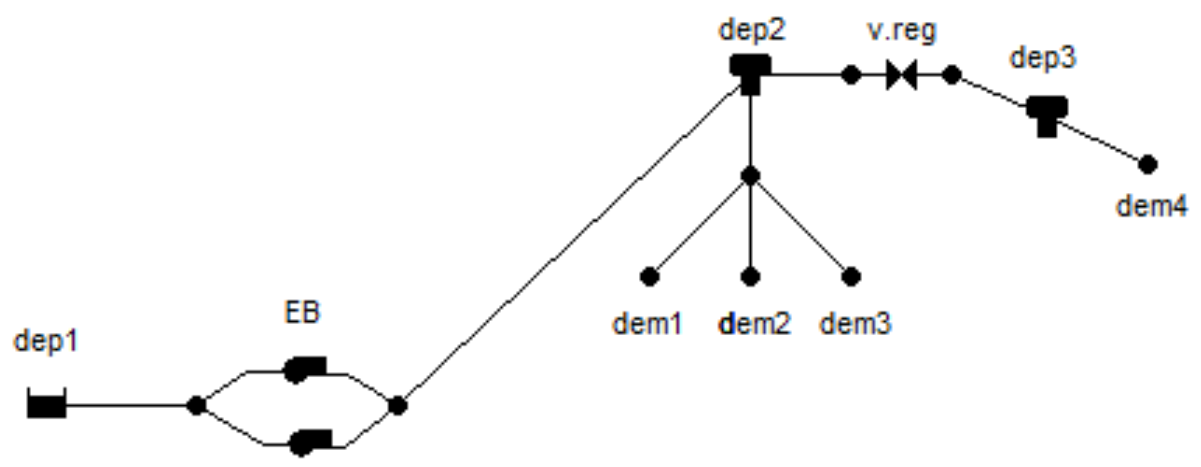

Figura 28. Esquema del subsistema a optimizar.

La estación de bombeo cuenta con dos bombas ITUR KSB de $37 \mathrm{~kW}$ de potencia nominal. Sin embargo, las bombas nunca trabajan simultáneamente, sino que se bombea con una u otra de manera alternativa. Así, si se produce una avería o debe realizarse el mantenimiento de una bomba, el sistema puede funcionar de igual modo. Ambas bombas cuentan con variador de velocidad, aunque no se utiliza para adecuar el caudal de manera variable con el tiempo, sino que se ha estipulado una velocidad de giro inferior a la nominal, la cual se aplica de modo constante cuando las bombas están en marcha.

El modo actual de funcionamiento del sistema se basa en la determinación de los instantes de arranque y paro de las bombas en función de unos determinados umbrales de nivel en los depósitos. Concretamente, se define el arranque de una de las bombas (la que menor tiempo de funcionamiento totalizado tenga) cuando el nivel del dep2 baja de 2,2 $\mathrm{m} \mathrm{y}$ su paro cuando el nivel alcanza los $3 \mathrm{~m}$. De igual modo, se determina la apertura de la válvula de regulación cuando el nivel del dep3 desciende hasta los $3 \mathrm{~m}$ y su cierre cuando el nivel alcanza los $3,7 \mathrm{~m}$.

\subsection{Resultados}

El escenario concreto que se propone para la evaluación del método de optimización basado en programación lineal consiste en el análisis de un periodo de 24 horas del pasado, en el que se conocen exactamente las demandas, así como las reglas de operación que efectivamente se llevaron a cabo. A partir de este escenario conocido, el método de 
optimización sugiere una estrategia distinta de operación buscando una reducción en el coste energético a la vez que se garantiza el cumplimiento de unas restricciones en los niveles de los depósitos.

El caso real de estudio tiene la limitación de que la válvula de regulación no permite un control alternativo al implementado. Así, el problema de optimización únicamente puede manejar como variables las dos bombas de la estación que controlan el trasiego de agua entre el dep1 y el dep2. Tanto el trasiego a través de la válvula de regulación como las demandas formarán parte de las condiciones de contorno del problema.

En concreto, se presenta el resultado para el día 1-6-21, aunque la misma comparativa se ha realizado para múltiples días, obteniendo porcentajes de mejora de entre el 5 y el $20 \%$. La Figura 29 muestra los caudales trasegados por los elementos de regulación en el escenario inicial, esto es, la simulación de las reglas de operación que se ejecutaron en la realidad.

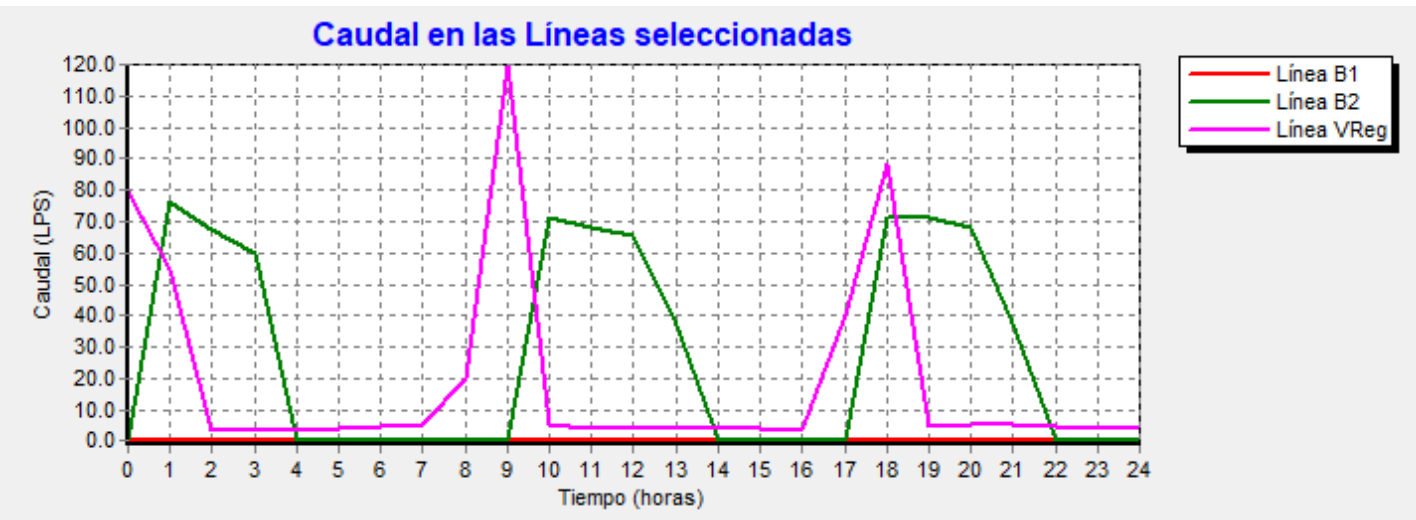

Figura 29. Caudal trasegado por las bombas B1 y B2, y por la válvula VReg en el escenario inicial que reproduce la operación real del día 1-6-21

La Figura 30 muestra la evolución del nivel en los depósitos que resulta de la simulación de las consignas de operación que se ejecutaron en realidad.

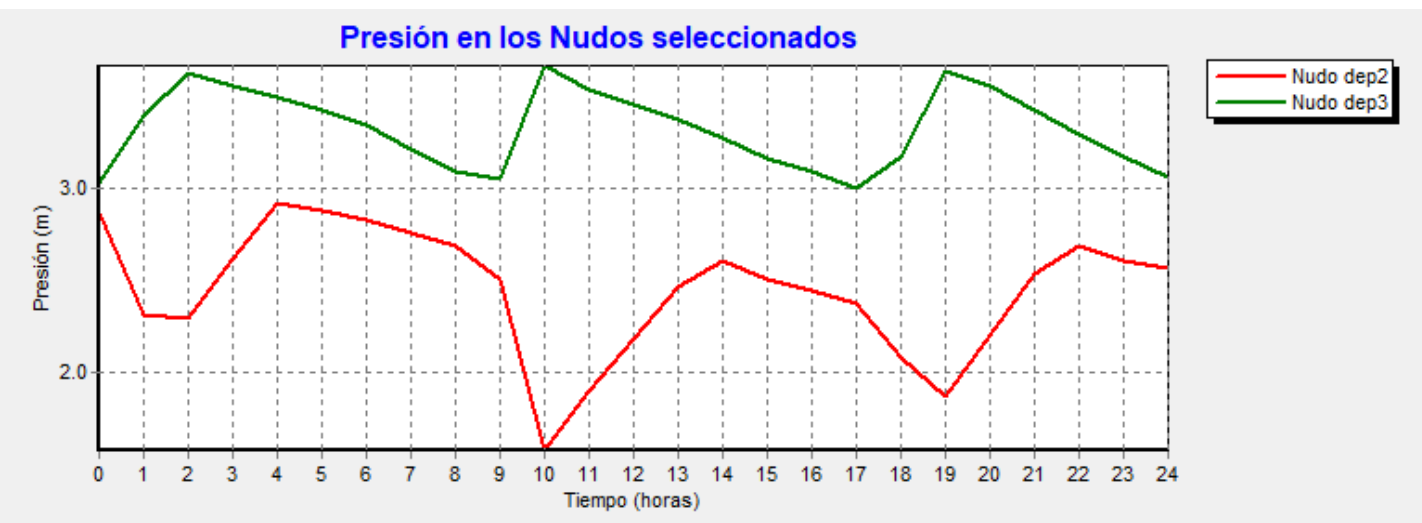

Figura 30. Evolución del nivel en los depósitos dep2 y dep3 en el escenario inicial que reproduce la operación real del día 1-6-21 
En la Figura 31 se muestra el precio final de la energía hora a hora para el día 1-6-21 según la tarifa eléctrica negociada por la compañía de distribución de agua.

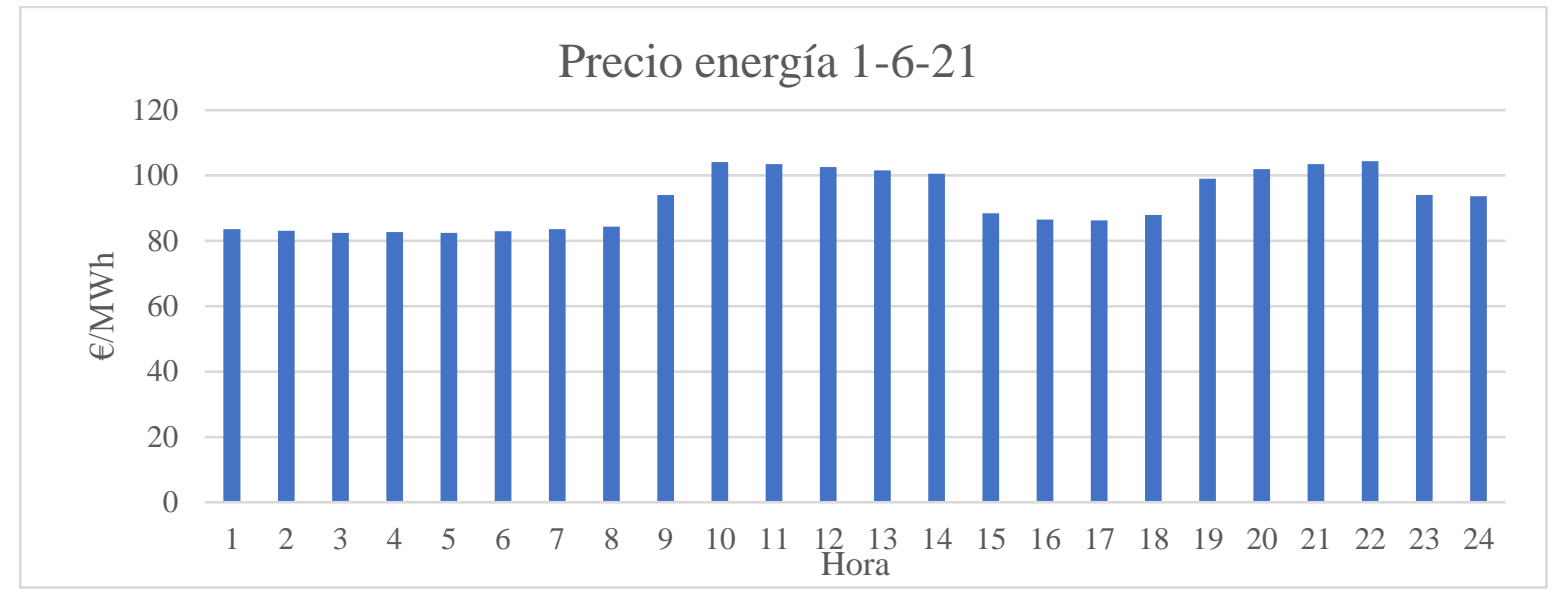

Figura 31. Precio de la energía para el día 1-6-21 ajustado a la tarifa negociada por la compañía gestora del sistema de distribución de agua.

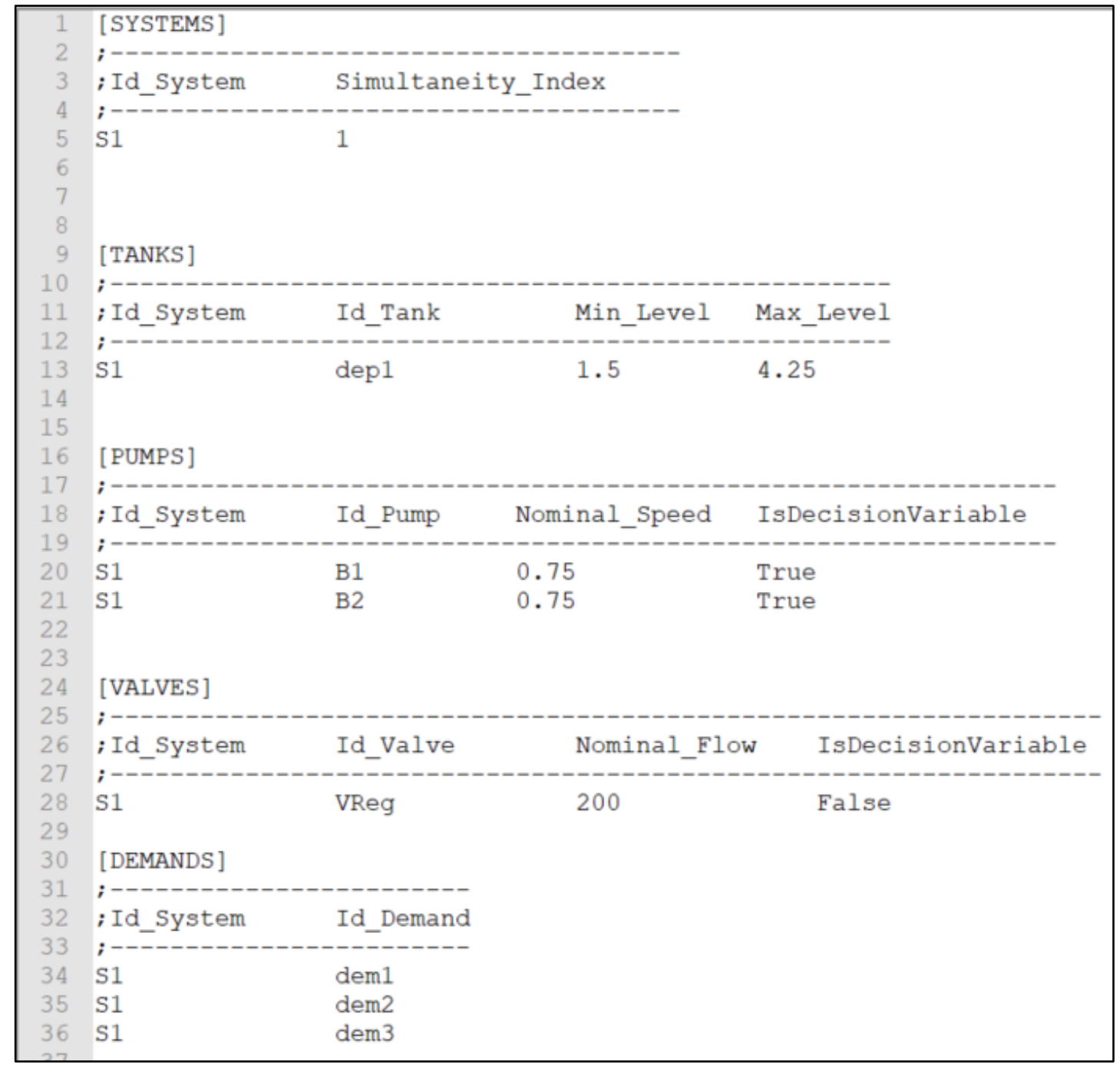

Figura 32. Configuración del subsistema a optimizar. 
La configuración del subsistema según la estructura definida en el apartado 4.2.1 se muestra en la Figura 32. Nótese que, al no poder considerar la válvula como variable de decisión, no es preciso incluir el dep3 en el subsistema.

Una vez configurados los parámetros para la optimización del subsistema, se ejecuta el algoritmo. El tiempo de computación en este caso es de $417 \mathrm{~ms}$, ejecutado con un procesador Intel Core i5 de $8^{\text {a }}$ generación. A continuación, se muestran los principales resultados.

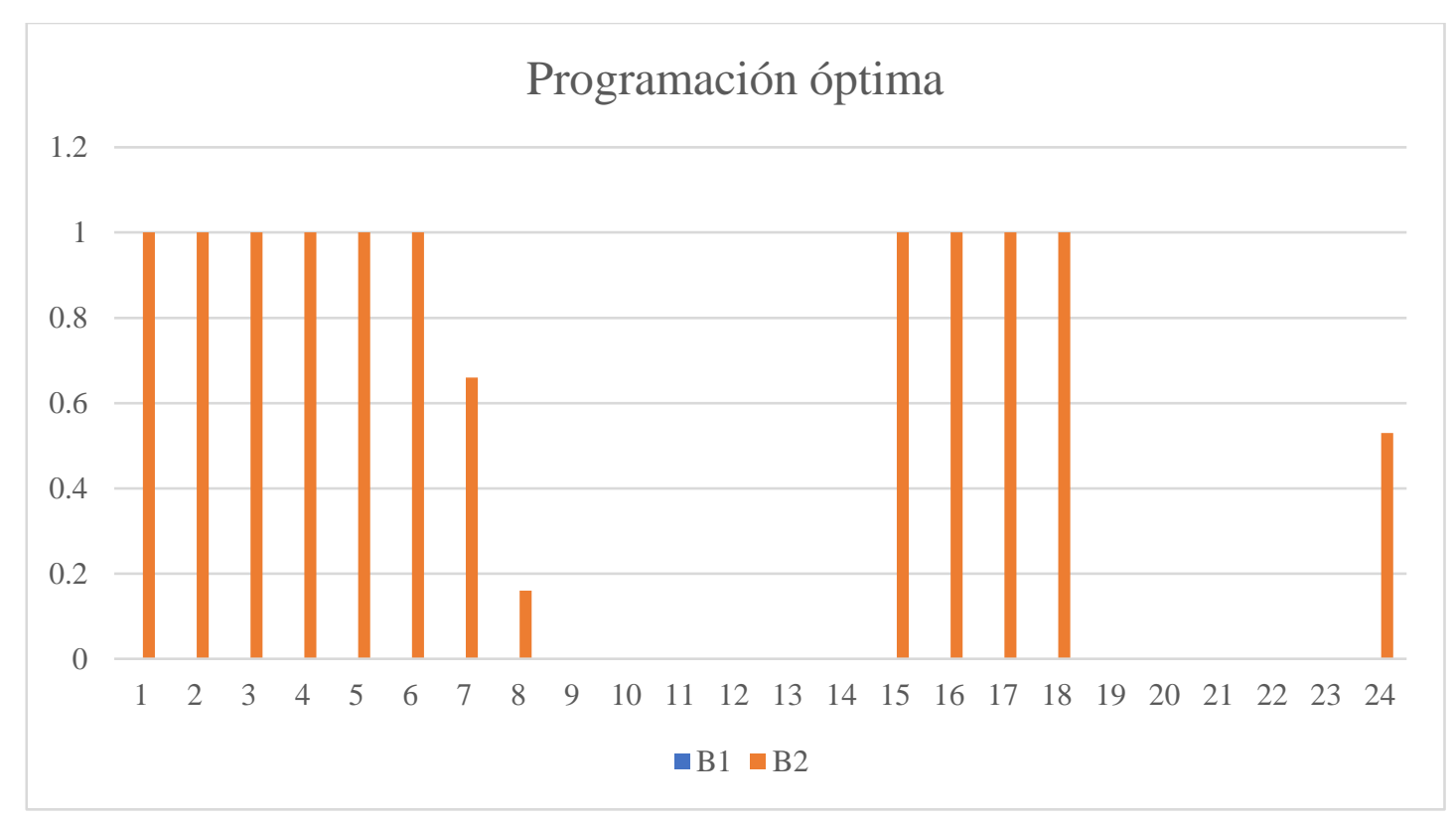

Figura 33. Resultado obtenido mediante programación lineal para la optimización del subsistema.

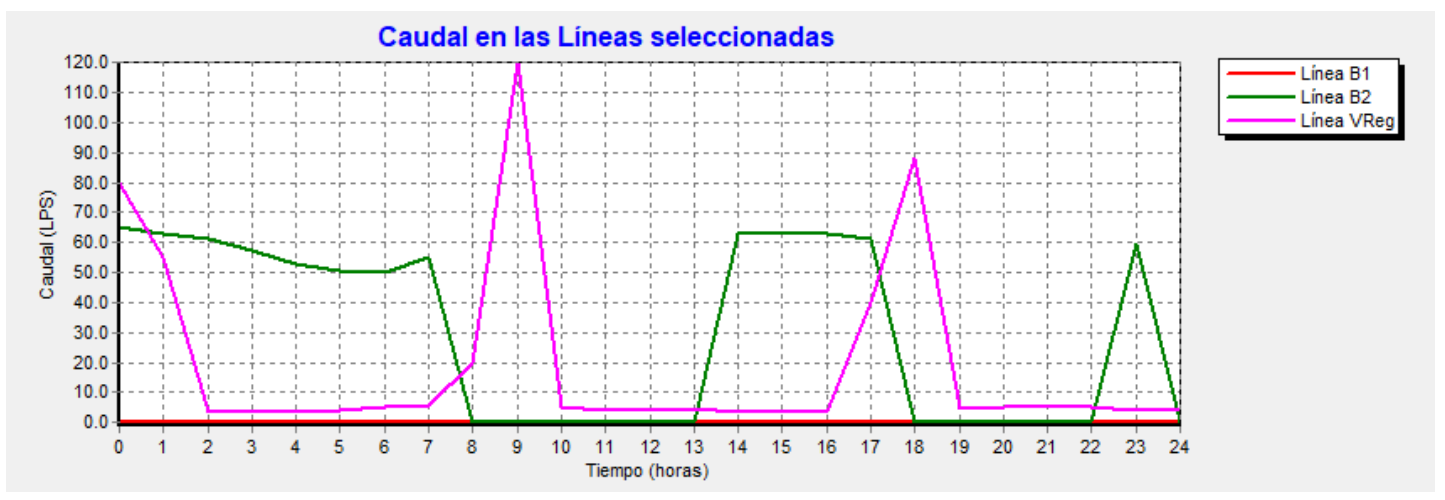

Figura 34. Caudal trasegado por las bombas B1 y B2, y por la válvula VReg en el escenario optimizado para el día 1-621 


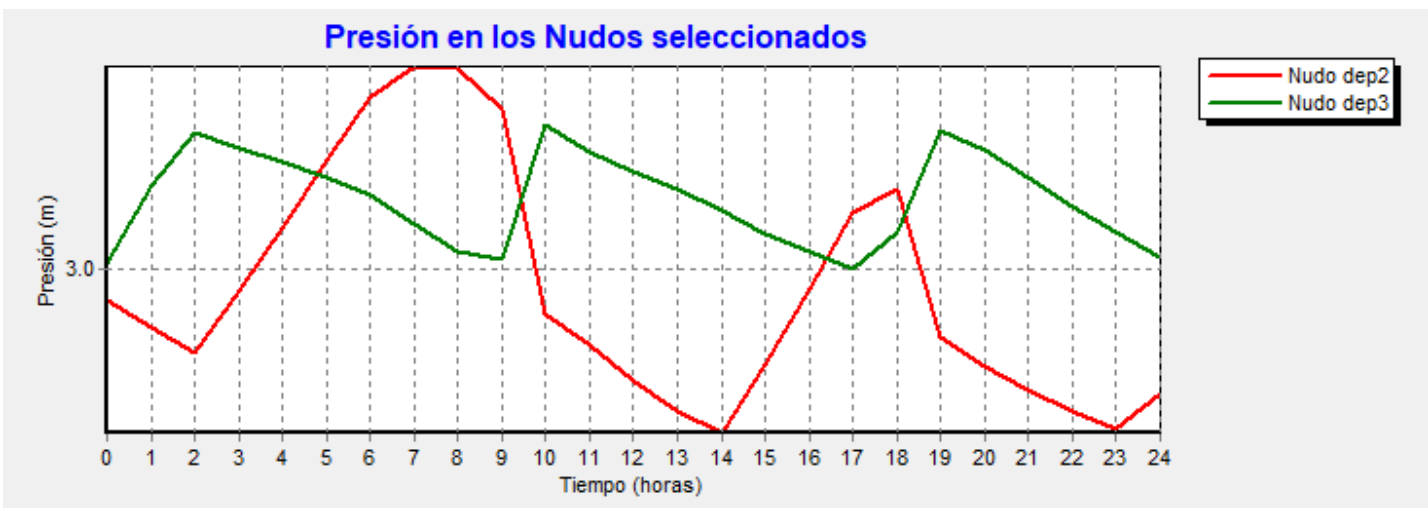

Figura 35. Evolución del nivel en los depósitos dep2 y dep3 en el escenario optimizado para el día 1-6-21

Tabla 9. Resumen comparativo entre los resultados de la simulación de la operación real y el escenario propuesto por el optimizador lineal.

\begin{tabular}{lll}
\hline & Escenario inicial & Escenario optimizado \\
\hline Volumen bombeado $\left(\mathrm{m}^{3}\right)$ & $2.494,92$ & $2.426,7$ \\
Energía consumida $(\mathrm{kWh})$ & 129,02 & 129,61 \\
Coste energía $(€)$ & 12,43 & 11,02 \\
Coste específico $\left(\mathrm{c} € / \mathrm{m}^{3}\right)$ & 0,4983 & 0,4542 \\
\hline
\end{tabular}

La ejecución de la programación propuesta por el método lineal de optimización ha dado como resultado una estrategia de control que lleva al cumplimiento de las restricciones de nivel en los depósitos, y a una mejora en la elección de los instantes en los que activar las bombas. En concreto, se ha alcanzado una mejora porcentual del 8,85\% en el coste específico respecto a la situación de partida. Cabe señalar que, con este enfoque, el consumo energético no tiene por qué reducirse, ya que la función objetivo responde al coste económico incurrido.

\subsection{Conclusiones}

La simplificación de las ecuaciones que definen el comportamiento hidráulico de los sistemas de transporte de agua puede no incurrir en errores excesivos en algunos casos y permiten una formulación del problema de optimización mediante métodos de programación lineal.

Estos métodos se diferencian respecto a otros métodos de optimización en que siempre garantizan el óptimo global, si existe, y en que son muy eficientes computacionalmente, resultando muy adecuados para su aplicación en la operación en tiempo real. 
En este capítulo se propone una formulación que permite la construcción sistemática del problema lineal a partir de un modelo hidráulico completo y una serie de parámetros configurables. El algoritmo calcula los coeficientes de la matriz a partir de los resultados obtenidos mediante la simulación hidráulica del modelo completo.

El método se ha aplicado con éxito a un caso de estudio real obteniendo reducciones en el coste específico diario del orden del 10\%. La eficiencia computacional ha quedado evidenciada, con un tiempo de computación inferior a 1 segundo para el caso de estudio descrito.

Como principales desventajas de este método respecto a los basados en algoritmos metaheurísticos o, como se verá en el siguiente capítulo, a algunos métodos heurísticos, cabe destacar que el error cometido en la simplificación puede conducir, no solo a un alejamiento del óptimo, sino incluso a la invalidez de la solución aportada. Además, este método no permite establecer como objetivo la minimización del consumo energético, ya que este se considera proporcional al volumen bombeado, sino que su objetivo se centra en minimizar el coste económico. 



\section{CAPÍtUlo 5.}

\section{MÉTOdOS HEURÍSTICOS}

\subsection{Introducción}

Según se ha visto en los capítulos anteriores, no existe un método general de optimización que se adapte perfectamente a los requerimientos del problema de minimización del consumo energético en la operación diaria de sistemas de distribución de agua. Cada uno de los métodos generales presenta una serie de ventajas y desventajas respecto a los demás.

Tal como se ha introducido en el apartado 2.1, los métodos heurísticos de optimización se basan en la definición de una regla construida a partir de un conocimiento profundo de un problema concreto, y permiten encontrar una aproximación a la solución del problema utilizando normalmente una estrategia voraz. Un excelente ejemplo de este tipo de métodos para la optimización de la programación de bombeo, que se basa en el concepto de “periodo de vaciado” se propuso por Pulido-Calvo y Gutiérrez-Estrada (2011).

En el presente capítulo se pretende explorar alguna de las posibilidades que existen en cuanto a la definición de una heurística que pueda ser de utilidad en la resolución de los problemas concretos aquí planteados. El objetivo final es el de encontrar una metodología acertada que permita alcanzar buenas soluciones con un esfuerzo computacional mínimo.

Así pues, se ha trabajado desde el nivel más básico del análisis energético de los sistemas de distribución de agua, para tratar de definir un nuevo modelo de cálculo de los flujos energéticos que ofrezca información detallada acerca del origen y del destino final de las aportaciones energéticas al sistema, con especial interés en los bombeos como fuentes de 
energía del sistema hidráulico, ya que son estos los que incurren en un coste para el gestor del SDA.

El nuevo modelo de cálculo finalmente permite la definición de un método heurístico que, como se verá en posteriores apartados, resulta eficaz para la optimización energética en tiempo real, ofreciendo algunas ventajas de las que carecen los métodos basados en algoritmos generales de optimización.

\subsection{Cálculo de los flujos energéticos en redes de distribución de agua}

\subsubsection{Descripción del método}

La metodología que se propone en el presente apartado se basa en el cálculo de un balance de energía instantáneo para cualquier sistema de distribución de agua, tomando como fuentes de energía las bombas y los embalses, estos últimos por lo que se refiere a la energía gravitatoria. Los sumideros de energía son los caudales salientes a través de los nudos, así como las pérdidas por fricción en las tuberías. Como primera aproximación al balance, los depósitos pueden ser considerados bien como fuentes de energía, en caso de que estén entregando caudal a la red, o bien como sumideros, en caso contrario. Para llevar a cabo el cálculo de los flujos energéticos a través de la red, es preciso formular un balance de energía a nivel de nudo, reto que no ha sido abordado con anterioridad en la bibliografía.

Uno de los puntos más destacables de la formulación que se presenta es que ninguno de los términos del balance depende del "datum" de la red, esto es, del nivel de referencia de las cotas. Así, el planteamiento finalmente supera las limitaciones que se derivan de los balances basados en alturas piezométricas.

\subsubsection{Balance global de energía en un instante dado}

El balance global de energía de un sistema de distribución de agua para un instante dado puede escribirse como:

$$
\gamma \sum_{j \in N} Q_{j} H_{j}+\sum_{p \in P} W_{p}-\sum_{l \in L} F_{l}=0
$$

donde $\gamma$ es el peso específico del agua, $Q_{j}$ es el caudal entrante o saliente a través del nudo $j, H_{j}$ es la altura piezométrica del mismo nudo, $W_{p}$ es la potencia hidráulica entregada por la 
bomba $p$ y $F_{l}$ es la potencia disipada por fricción en la línea $l$, incluyéndose aquí tanto las pérdidas por fricción en las tuberías como las pérdidas de carga en las válvulas.

El primer sumatorio se extiende a todos $\operatorname{los}$ nudos $N$ del sistema, incluyendo embalses, depósitos y nudos de demanda. Se asume que el caudal entra al sistema a través de los embalses $\left(Q_{r}>0\right)$ y que sale de él a través de los nudos de demanda $\left(Q_{d}<0\right)$. Nótese que el caudal saliente responde tanto a demandas contabilizadas como a fugas. Los depósitos, por su parte, pueden recibir caudal, entregarlo o ambas situaciones simultáneamente en caso de disponer de tuberías separadas de entrada y salida $\left(Q_{t}<0\right.$ o $Q_{t}>0$ ). En el análisis que se propone aquí, los caudales entrantes y salientes se tratan por separado, de modo que $Q_{t}=Q_{t o}-Q_{t i}$, donde $Q_{t o}$ representa el caudal total saliente del depósito en un instante y $Q_{t i}$ el caudal total entrante. Si $R$ denota al conjunto de embalses del sistema, $T$ indica el número de depósitos y $D$ es el número de nudos de demanda, siendo $N=R \cup T \cup D$, el primer sumatorio de la Ecuación (42) puede descomponerse como:

$$
\gamma \sum_{j \in N} Q_{j} H_{j}=\gamma \sum_{r \in R} Q_{r} H_{r}+\gamma \sum_{t o \in T} Q_{t o} H_{t o}-\gamma \sum_{t i \in T} Q_{t i} H_{t i}-\gamma \sum_{d \in D} Q_{d} H_{d}
$$

donde los primeros dos términos de la derecha de la igualdad representan la cantidad de energía gravitatoria aportada al sistema a través de los embalses y depósitos, y los dos últimos términos representan la energía recibida en los depósitos y nudos de demanda.

El segundo sumatorio de la Ecuación (42) está extendido a todas las bombas $P$ que aportan energía al sistema, ya sean bombas de pozo, estaciones elevadoras o bombas sobrepresoras. La expresión de la potencia aportada por la bomba $p$ es $W_{p}=\gamma Q_{p} \Delta H_{p}>0$, siendo $Q_{p}$ el caudal total que pasa por la misma y $H_{p}$ la altura manométrica comunicada. Si en el sistema hubiera turbinas para aprovechar la caída de presión en algún punto, entonces sería $\Delta H_{p}<0 \mathrm{y}$, en consecuencia $W_{p}$ sería menor que cero.

Finalmente, el tercer sumando de la Ecuación (42) está extendido a todas las tuberías y válvulas $L$ del sistema en las que se disipa energía por fricción al paso del flujo, de modo que $F_{l}=\gamma Q_{l} h_{l}$, siendo $Q_{l}$ el caudal de la línea y $h_{l}$ la pérdida de carga en la misma.

La Ecuación (43) se puede modificar si se tiene en cuenta que $H_{j}=z_{j}+p_{j} / \gamma$, donde $p_{j}$ es la presión en el nudo $j$ y $z_{j}$ su elevación respecto a un nivel de referencia (datum de la red). Puesto que la presión en la lámina libre del agua en embalses y depósitos es cero, 
sustituyendo el primer sumatorio de la Ecuación (42) por la parte derecha de la igualdad de la Ecuación (43), y reordenando, la Ecuación (42) resulta:

$$
\begin{gathered}
\left(\gamma \sum_{r \in R} Q_{r} z_{r}+\gamma \sum_{t o \in T} Q_{t o} z_{t o}\right)-\left(\gamma \sum_{t i \in T} Q_{t i} z_{t i}+\gamma \sum_{d \in D} Q_{d} z_{d}\right) \\
+\gamma \sum_{p \in P} Q_{p} \Delta H_{p}-\gamma \sum_{l \in L} Q_{l} h_{l}=\gamma \sum_{d \in D} Q_{d} \frac{p_{d}}{\gamma}
\end{gathered}
$$

Puesto que el balance de caudales debe cumplirse en cualquier instante:

$$
\left(\sum_{r \in R} Q_{r}+\sum_{t o \in T} Q_{t o}\right)-\left(\sum_{t i \in T} Q_{t i}+\sum_{d \in D} Q_{d}\right)=0
$$

puede probarse que el hecho de añadir una constante a todas las elevaciones $z$ no va a modificar el balance de energía anterior, de modo que este es independiente del origen de cotas que se tome como referencia, tal como se ha indicado anteriormente.

La Ecuación (44) indica que toda la energía de presión que se entrega en los nudos de demanda depende de las cotas de estos, de los caudales y cotas de las fuentes de suministro, de la energía aportada por las bombas o consumida por las turbinas y de la energía disipada por fricción en tuberías y válvulas.

Con el objetivo de simplificar el análisis posterior, el conjunto de embalses $R$ y el de depósitos que entregan caudal a la red $T_{o}$ se van a fusionar en el nuevo conjunto $S$, entendido como la agrupación de las fuentes de agua del sistema en el instante de estudio. Del mismo modo, el conjunto de nudos de demanda $D$ y de depósitos con caudal entrante $T_{i}$ se fusionan en el nuevo conjunto $C$ que representa los puntos de consumo en ese mismo instante. Así, la Ecuación (44) puede reescribirse como:

$$
\gamma\left(\sum_{s \in S} Q_{s} z_{s}-\sum_{c \in C} Q_{c} z_{c}\right)+\gamma \sum_{p \in P} Q_{p} \Delta H_{p}-\gamma \sum_{l \in L} Q_{l} h_{l}=\gamma \sum_{c \in C} Q_{c} \frac{p_{c}}{\gamma}
$$

El siguiente reto consiste en la formulación de este balance para cada nudo del sistema individualmente.

\subsubsection{Balance de energía en un nudo para un instante dado}

Tal como se ha avanzado, para proponer un balance de energía en un nudo se parte de la suposición de que en un instante dado el conjunto de fuentes de agua $S$ lo forman los 
embalses y los depósitos con caudal saliente. De modo similar, el conjunto de consumos de agua $C$ lo forman los nudos de demanda y los depósitos con caudal entrante. Ahora bien, si en algún depósito se da la situación de que simultáneamente entra y sale caudal a través de tuberías diferenciadas, en este caso se desdoblaría el depósito en dos depósitos idénticos, pasando uno a formar parte de las fuentes de agua y el otro de los consumos.

Si $Q_{c}$ es el caudal total demandado en el punto $c$ (ya sea demanda o depósito con caudal entrante), y $q_{c, s}$ es la parte del caudal entregado a dicho punto desde la fuente de agua $s$ (ya sea un embalse o un depósito con caudal saliente), entonces:

$$
\sum_{s \in S} q_{c, s}=Q_{c}
$$

Por otro lado, si $W_{c}$ es la fracción de la energía total entregada por las bombas que llega al punto de consumo $c, \mathrm{y} w_{c, p}$ es la fracción de la energía aportada por la bomba $p$ y que llega al punto $c$, de no haberse disipado, entonces:

$$
\sum_{p \in P} w_{c, p}=W_{c}
$$

Debe remarcarse que, en caso de existir turbinas para la recuperación de energía, estas pertenecerían al conjunto de bombas, aunque su contribución sería negativa.

Análogamente, si $F_{C}$ es la fracción de la energía total disipada en el sistema que habría llegado al punto $c$ de no haberse disipado, y $f_{l, c}$ es la fracción de la energía disipada en línea $l$ y que habría llegado al punto $c$, entonces:

$$
\sum_{l \in L} f_{c, l}=F_{c}
$$

De acuerdo con la Ecuación (44) y teniendo en cuenta las relaciones dadas por las ecuaciones (48) y (49), el balance de energía para un determinado punto de consumo $c$ y un instante dado puede expresarse como: 


$$
\gamma \sum_{s \in S} q_{c, s}\left(z_{s}-z_{c}\right)+\sum_{p \in P} w_{c, p}-\sum_{l \in L} f_{c, l}=\gamma Q_{c} \frac{p_{c}}{\gamma}
$$

La Ecuación (50) muestra que la energía gravitatoria o potencial que se entrega en un punto de consumo depende del caudal procedente de cada una de las fuentes de agua y de la diferencia de cota con respecto a ellas.

Finalmente, realizando el sumatorio de la Ecuación (50) para todos los puntos de consumo se tiene la siguiente expresión:

$$
\gamma \sum_{\mathrm{c} \in \mathrm{C}} \sum_{s \in S} q_{c, S}\left(z_{S}-z_{c}\right)+\sum_{c \in C} \sum_{p \in P} w_{c, p}-\sum_{c \in C} \sum_{l \in L} f_{c, l}=\gamma \sum_{\mathrm{c} \in \mathrm{C}} Q_{c} \frac{p_{c}}{\gamma}
$$

Dado que el orden de los sumatorios puede invertirse y atendiendo a las ecuaciones (47), (48), (49) y (50), se puede concluir que la Ecuación (51) coincide con la expresión para el balance de energía global (46).

En este apartado se ha propuesto una ecuación de equilibrio de energía a nivel de nudo, y se ha mostrado su equivalencia con la ecuación de balance global cuando se agrega para el conjunto de puntos de consumo. Sin embargo, queda definir un método que permita el cálculo de las fracciones de caudal $q_{c, s}$, así como las fracciones de energía aportada por las bombas $w_{c, p}$, y las fracciones de pérdidas de carga $f_{c, l}$.

\subsubsection{Determinación de las fracciones de caudal y energía en cada punto de consumo} procedentes de cada fuente

Se propone en este apartado un método para la obtención de las fracciones de caudal $q_{c, s}$, fracciones de energía aportada $w_{c, p}$ y disipada $f_{c, l}$ que se basa en un reparto proporcional a los caudales de las líneas incidentes a cada nudo del sistema. El método debe garantizar que las fracciones obtenidas conduzcan al cumplimiento de la Ecuación (51), es decir, las presiones nodales que se obtengan por este método deben coincidir con las calculadas mediante la resolución de las ecuaciones hidráulicas de la red.

\section{Fracciones de caudal}

El método que se desarrolla a continuación parte de que el caudal de cada línea del sistema es conocido, debiéndose obtener previamente mediante la resolución de las ecuaciones hidráulicas para el instante considerado. 
Se establece como premisa que el caudal total $Q_{l}$ que circula por la línea $l$ puede descomponerse en tantos caudales fraccionarios $q_{l, s}$ como fuentes de agua $s$ se consideren, pudiéndose expresar cada fracción del caudal mediante la siguiente expresión:

$$
q_{l, s}=p_{l, s} Q_{l}
$$

donde $p_{l, s}$ es la proporción del caudal de la línea $l$ que procede de la fuente $s$ (Figura 36) de tal modo que, para cada línea se cumplen las siguientes dos condiciones:

$$
\begin{aligned}
& \sum_{s \in S} p_{l, s}=1 \\
& \sum_{s \in S} q_{l, s}=Q_{l}
\end{aligned}
$$

Para un punto de consumo $c$ se define $I_{c}$ como el conjunto de las líneas incidentes a dicho punto con caudal entrante, y $O_{c}$ como el conjunto de líneas incidentes con caudal saliente. Si $p_{c, s}$ es la proporción del caudal que llega al punto $c$ procedente de la fuente $s$, asumiendo la hipótesis de mezcla perfecta de los flujos entrantes a cada nodo, entonces:

$$
p_{c, s}=\frac{\sum_{l \in I_{c}} q_{l, s}}{\sum_{s \in S} \sum_{l \in I_{c}} q_{l, s}}=\frac{\sum_{l \in I_{c}} q_{l, s}}{\sum_{l \in I_{c}} Q_{l}}
$$

donde el numerador es la suma de todos los flujos procedentes de $s$ que llegan a $c$, y el denominador es la suma de todos los flujos entrantes a $c$, independientemente de su origen.

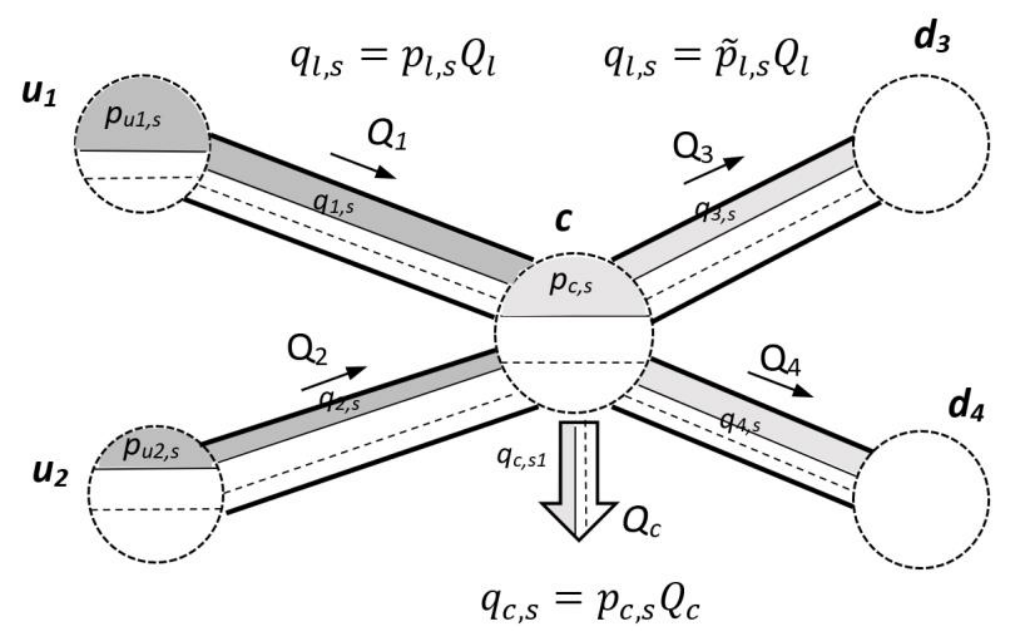

Figura 36. Modelo de mezcla en el punto c de las fracciones de caudal procedentes de la fuente s 
Se asume también que la proporción $p_{c, s}$ en un nudo es compartida por todas las líneas con caudal saliente (Figura 36), es decir, que para el conjunto $O_{c}$ de líneas, $p_{l . s}=p_{c, s}$, de modo que:

$$
q_{l, s}=p_{l . s} Q_{l}=p_{c, s} Q_{l} \quad \forall l \in O_{c}
$$

Del mismo modo, para la demanda $Q_{c}$ de ese nodo se tiene:

$$
q_{c, s}=p_{c, s} Q_{c}
$$

Nótese que $p_{c, s}$ es una variable referida a los nudos mientras que $p_{l, s}$ es una variable referida a las líneas. Sin embargo, a la vista de la Ecuación (56), las fracciones de caudal en cada línea $q_{l, s}$ pueden expresarse en base a las proporciones obtenidas para el nudo ubicado aguas arriba de la misma. Así, de manera general, si se define $\tilde{p}_{l, s}$ como la variable nodal correspondiente al nudo aguas arriba de la línea $l$, esto permitirá formular un sistema de ecuaciones cuyas incógnitas son exclusivamente las proporciones nodales. Teniendo en cuenta que $p_{l, s}=\tilde{p}_{l, s}$, y que el caudal total entrante a un nudo debe coincidir con el caudal total saliente:

$$
\sum_{l \in I_{c}} Q_{l}=\sum_{l \in O_{c}} Q_{l}+Q_{c}
$$

entonces, la Ecuación (55) puede reescribirse como:

$$
p_{c, s}\left(\sum_{l \in O_{c}} Q_{l}+Q_{c}\right)-\sum_{l \in I_{c}} \tilde{p}_{l, s} Q_{l}=0
$$

Si se formula la Ecuación (59) para todos los nudos del sistema y se tiene en cuenta que en los nudos correspondientes a las fuentes de agua se debe cumplir que $p_{c, s}=1$ si $c=$ $s$, y $p_{c, s}=0$ si $c \neq s^{3}$, se puede plantear un sistema de ecuaciones lineal cuya resolución

\footnotetext{
${ }^{3}$ Aquí se ha reutilizado por simplicidad para las fuentes de agua la misma nomenclatura definida para puntos de consumo.
} 
proporcionará los valores de las proporciones en todos los puntos de consumo $p_{c, s}$, así como también indirectamente las proporciones en las líneas $p_{l, s}$ (dado que $p_{l, s}=\tilde{p}_{l, s}$ ).

El sistema de ecuaciones lineal puede plantearse de forma matricial como:

$$
\boldsymbol{A P} \boldsymbol{P}_{s}=\boldsymbol{Q}_{s}
$$

donde $\boldsymbol{A}$ es una matriz cuadrada de dimensiones $(C+S) x(C+S)^{4}$. Cada fila representa el planteamiento de la Ecuación (59) para un nudo concreto $n$. Los coeficientes de la diagonal se corresponden con la suma de caudales salientes de cada nudo $n$ (tanto a través de líneas como por demandas externas). El resto de los coeficientes hacen referencia al nudo aguas arriba de cada una de las tuberías con caudal entrante en $n$, y toman el valor de cero en las columnas correspondientes a los nudos no conectados aguas arriba, y el valor del caudal que circula por cada tubería entrante, cambiado de signo, en las columnas correspondientes al nudo aguas arriba de cada tubería.

El vector $\boldsymbol{P}_{S}$ contiene las incógnitas del problema, que indicarán, para cada nudo del sistema $n$, la proporción de agua procedente de la fuente $s$ que llega a $n$. Finalmente, $\boldsymbol{Q}_{s}$ es un vector cuyos elementos son cero, excepto el elemento situado en la fila correspondiente a la fuente de agua $s$, que coincide con el caudal total entregado por la fuente.

La matriz de coeficientes $\boldsymbol{A}$ se puede expresar también en función de las matrices de incidencia y de caudales de línea como:

$$
A=\Lambda Q_{l} \Lambda_{d}^{T}+Q_{c}
$$

donde $\boldsymbol{Q}_{\boldsymbol{l}}$ es una matriz diagonal $L x L$ que contiene los caudales de línea en valor absoluto sobre los elementos de la diagonal. $\Lambda$ es la matriz de incidencia $(C+S) x L$ (Todini y Pilati, 1988) del grafo no dirigido asociado a la red hidráulica, que identifica los nudos aguas arriba y aguas abajo de cada línea según el sentido de los caudales, de modo que:

- $\quad \lambda_{n, l}=1$; si la línea $l$ sale del nudo $n$.

\footnotetext{
${ }^{4}$ Obsérvese que la dimensión de la matriz responde a todos los nudos de la red, independientemente de que se trate de fuentes de suministro o de puntos de consumo. Así, pueden imponerse fácilmente las condiciones de contorno que determinan las proporciones en los propios puntos de suministro.
} 
- $\quad \lambda_{n, l}=-1$; si la línea $l$ entra al nudo $n$.

- $\quad \lambda_{n, l}=0$; si la línea $l$ y el nudo $n$ no están conectados.

$\boldsymbol{\Lambda}_{\boldsymbol{d}}$ es la matriz de incidencia $(C+S) x L$ del grafo dirigido según el sentido de los caudales, que únicamente identifica el nudo aguas arriba de cada línea, de modo que:

- $\quad \lambda_{n, l}=1$; si la línea $l$ sale del nudo $n$.

- $\quad \lambda_{n, l}=0$; si la línea $l$ entra al nudo $n$ o si $l$ y $c$ no están conectados.

Por último, $\boldsymbol{Q}_{\boldsymbol{c}}$ es una matriz diagonal $(C+S) x(C+S)$ que contiene los caudales salientes a través de los nudos de consumo (tanto demandas como depósitos con caudal entrante) en las posiciones diagonales.

La Ecuación matricial (60) puede generalizarse con el objetivo de incluir a todas las fuentes de suministro simultáneamente, de modo que:

$$
\boldsymbol{A P}=\boldsymbol{Q}
$$

donde $\boldsymbol{P}$ es ahora una matriz $(C+S) x S$ que contiene las proporciones desde cada fuente en la columna correspondiente, y $\boldsymbol{Q}$ es otra matriz de la misma dimensión con los caudales inyectados por cada fuente aplicados en el nudo, fila y columna, correspondiente. La matriz $\boldsymbol{A}$ es común a todas las fuentes.

Puesto que las fuentes son el origen al agua que fluye por la red, los valores $p_{c, s}$ que representan la proporción de agua con origen en $s$ que llega al nudo $c$, serán conocidos y constantes en las propias fuentes, siendo $p_{c, s}=1$ cuando $c=s$, y $p_{c, s}=0$ en caso contrario. Una vez calculadas las proporciones $p_{c, s}$ para todos los nudos de consumo y fuentes de agua, los valores de $q_{c, s}$ para cada nudo pueden calcularse a través de la Ecuación (57) y, por lo tanto, ya puede calcularse el primer término de la Ecuación (51), que representa el balance de energía a nivel de nudo para un instante dado. Este primer término del balance representa la energía potencial o gravitatoria que llega a los nudos desde cada fuente de agua del sistema en un instante dado, incluyendo los embalses y depósitos que inyectan agua a la red. Nótese 
que la energía gravitacional podría ser negativa si las cotas de las fuentes de agua fueran inferiores a las de los puntos de consumo.

Para completar la evaluación de la Ecuación (51), debe proponerse también un método de cálculo para obtener las fracciones de energía $w_{c, p}$ que llegan a los nudos de consumo desde las distintas fuentes de energía (bombas), así como la asociación de las pérdidas por fricción en las tuberías con los nudos de consumo $f_{c, l}$.

\section{Fracciones de energía aportada}

Mientras que la distribución de la energía gravitacional depende (además de la distribución de los caudales en las líneas) de la diferencia de cota entre el origen y el destino del agua, la distribución de la energía aportada mediante bombas o disipada por fricción depende únicamente de la distribución de los flujos, y de la altura o pérdida en cada elemento, siendo este tipo de energía es independiente de las cotas de los nudos intermedios y extremos.

Para el seguimiento del transporte de energía, se propone expresar la distribución de energía en función de la potencia que se transmite a través de las líneas instantáneamente, es decir, la energía transmitida por cada línea por unidad de tiempo.

La potencia hidráulica que se transmite a un flujo de agua cuando pasa a través de una bomba $p$ tiene como expresión $W_{p}=\gamma Q_{p} \Delta H_{p}$, donde $Q_{p}$ es el caudal que trasiega la bomba y $\Delta H_{p}$ es la energía específica, o energía por unidad de peso, entregada al fluido. Se asume a continuación que la energía se transporta por filetes, diferenciados en función de la distribución de caudales que pasan por la bomba.

Se puede ilustrar simbólicamente asumiendo que la energía que recibe el fluido viaja a través de tubos de corriente que salen de cada bomba y viajan aguas abajo a través de los elementos de la red. Cuando un tubo de corriente llega a un nudo se divide en tantos otros tubos de corriente como líneas parten del nudo, sin mezclarse con el agua que viene de otras fuentes.

El análisis de este apartado requiere la introducción de una variable intensiva, que se ha denominado densidad de energía, y que responde a la cantidad de energía por unidad de volumen que se ha transportado hasta un determinado punto desde una fuente de energía. Por ejemplo, el flujo que atraviesa una bomba adquirirá una densidad de energía $e_{p}=$ 
$W_{p} / Q_{p}$. Si la potencia hidráulica entregada por la bomba se expresa en $k W$ y el caudal trasegado en $m^{3} / h$, entonces esta densidad de energía se medirá en $\mathrm{kWh} / \mathrm{m}^{3}$.

La principal hipótesis en la que se basa el método es que la densidad de energía $e_{p}$ que se transmite desde una bomba $p$ se mantiene constante a lo largo de los tubos de corriente que distribuyen dicha energía a través de la red. La fracción de esta energía que es transportada por una tubería situada aguas abajo de la bomba se obtiene, en consecuencia, como:

$$
w_{l, p}=e_{p} q_{l, p}
$$

donde $q_{l, p}$ es la fracción de caudal a través de la línea $l$ procedente del caudal impulsado por la bomba $p$. Dicha fracción se define de modo análogo a lo expuesto en el punto anterior, pero reemplazando la fuente de agua $s$ por la bomba $p$ (Figura 37). Además, si una parte $Q_{c, p}$ de este caudal sale del sistema, la fracción de energía procedente de la bomba $p$ que consumiría el nudo $c$ sería:

$$
w_{c, p}=e_{p} Q_{c, p}
$$

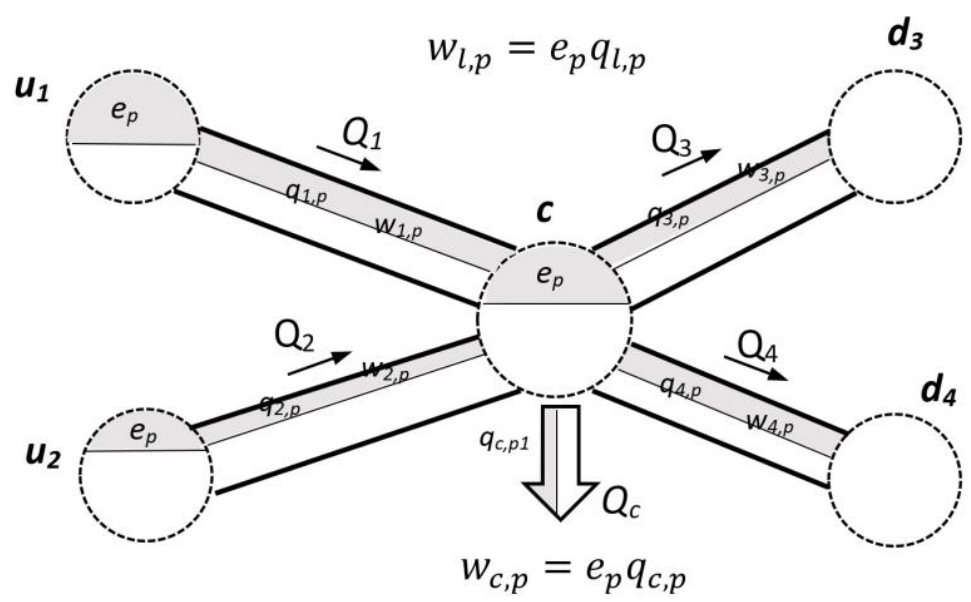

Figura 37. Modelo de mezcla en el punto de consumo c para la energía procedente de la bomba p, transportada a lo largo de un tubo de corriente con una densidad energética constante $e_{p}$

Nótese que $e_{p}$ es una variable nodal que permanece constante en todos los nudos aguas abajo de la bomba $p$. Por otra parte, un determinado nudo puede asociarse con varios valores de $e_{p}$ (o con ninguno), en función del número de bombas que se ubiquen aguas arriba del nudo y que le aporten agua y, por tanto, energía. 
Esta formulación es general y no depende del tipo de bomba (alimentación, sobrepresora, etc.) ni de su ubicación con respecto a otras bombas (serie, paralelo o independiente).

De acuerdo al balance energético, para cualquier nudo de consumo $c$, debe cumplirse la siguiente expresión:

$$
\sum_{l \in I_{c}} w_{l, p}=\sum_{l \in O_{c}} w_{l, p}+w_{c, p}
$$

la cual es equivalente a la ecuación de balance de caudales, ya que la densidad de energía $e_{p}$ permanece constante en todos los tubos de corriente que parten de la bomba $p$.

La fracción de energía transportada por cualquiera de las líneas salientes de un determinado nudo en relación a la energía total procedente de la bomba $p$ y que entra en el nudo, puede también escribirse como:

$$
\frac{w_{l, p}}{\sum_{l \in I_{c}} w_{l, p}}=\frac{e_{p} q_{l, p}}{e_{p} \sum_{l \in I_{c}} q_{l, p}}=\frac{q_{l, p}}{\sum_{l \in I_{c}} q_{l, p}}=\frac{Q_{l}}{\sum_{l \in I_{c}} Q_{l}}
$$

puesto que los caudales salientes se distribuyen siguiendo la misma proporción independientemente de su origen. Esta última igualdad resulta decisiva para justificar el procedimiento que se explica a continuación.

Se define una nueva variable $e_{c, p}$ que representa la densidad energética en el nudo $c$ procedente de la bomba $p$ como:

$$
e_{c, p}=\frac{\sum_{l \in I_{c}} w_{l, p}}{\sum_{l \in I_{c}} Q_{l}}
$$

donde el numerador responde a la energía total procedente de la bomba $p$ que llega al nudo $c$ en un determinado instante, y el denominador indica el caudal total que entra en dicho nudo. A diferencia de la variable $e_{p}$ definida con anterioridad, la nueva definición de densidad energética que se propone en la Ecuación (67) no es idéntica para todos los nudos (Figura 38). Así, se redefine la expresión que determina la fracción de energía que es transportada por una tubería como:

$$
w_{l, p}=\tilde{e}_{l, p} Q_{l}
$$


siendo $\tilde{e}_{l, p}$ la variable nodal asociada al nudo aguas arriba de la línea $l$ y que coincide con la variable referida a la línea $e_{l, p}$.

Los consumos externos de energía se determinan finalmente como:

$$
w_{c, p}=e_{c, p} Q_{c}
$$

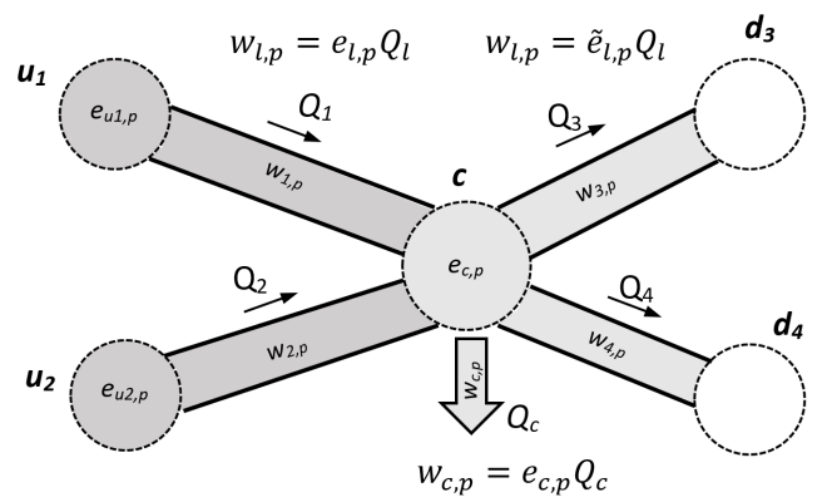

Figura 38. Modelo de mezcla en el nudo de consumo c para la energía procedente de la bomba p transportada por el caudal total, con distinta densidad energética para cada nudo.

Esta formulación presenta la ventaja de ser independiente de las fracciones de caudal con origen en la bomba $p$, así que no se precisa su cálculo.

El valor de esta nueva variable en cada nudo de consumo será calculado de un modo similar al planteado en la sección anterior:

$$
e_{c, p}=\frac{\sum_{l \in I_{c}}\left(\tilde{e}_{l, p} Q_{l}+W_{l, p}\right)}{\sum_{l \in O_{c}} Q_{l}+Q_{c}}
$$

donde $\tilde{e}_{l, p}$ es la variable nodal que indica la densidad energética del nudo aguas arriba de la línea $l, \mathrm{y} W_{l, p}$ es la potencia hidráulica aportada por la bomba $p$, la cual valdrá cero para todas las líneas excepto para aquellas que representan una bomba. La Ecuación (70) planteada para todos los nudos del sistema, conduce a un sistema lineal de ecuaciones que puede expresarse matricialmente como:

$$
\boldsymbol{A} \boldsymbol{E}_{p}=\boldsymbol{W}_{p}
$$

donde $\boldsymbol{A}$ es la misma matriz definida en la sección anterior, $\boldsymbol{E}_{p}$ es el vector de incógnitas que identificará las densidades energéticas referentes a la bomba $p$ en todos los nudos del 
sistema, y $\boldsymbol{W}_{p}$ es un vector que contiene el valor de la potencia hidráulica transmitida al fluido por la bomba $p$ en la fila que identifica al nudo aguas abajo de la misma bomba, siendo cero el resto de los elementos del vector. Se puede intuir que las densidades energéticas que resulten serán cero para los nudos aguas arriba de la bomba y tendrán un valor positivo y distinto para cada nudo ubicado aguas abajo.

Una vez se determinan las densidades energéticas para los nudos, puede calcularse la potencia procedente de la bomba $p$ que transporta cada línea mediante la Ecuación (68), y la potencia que sale de la red por los nudos de consumo mediante la Ecuación (69). Estos últimos valores corresponden a la variable $w_{c, p}$ que es la que aparece en la ecuación de balance en energía a nivel de nudo.

Puesto que la variable $e_{c, p}$ no depende de la distribución de los caudales procedentes de cada bomba, sino de los caudales totals de cada línea, se pueden calcular simultáneamente las densidades energéticas debidas a todas las bombas del sistema mediante la resolución del sistema matricial siguiente:

$$
\boldsymbol{A E}=\boldsymbol{W}
$$

donde $\boldsymbol{E}$ y $\boldsymbol{W}$ son ahora matrices con tantas columnas como bombas hay en el sistema, y cada columna se construye tal como se han definido $\boldsymbol{E}_{p}$ y $\boldsymbol{W}_{p}$.

Puesto que el denominador de la Ecuación (70) es común, es posible superponer la potencia transmitida por varias bombas, pudiéndose agrupar, por ejemplo, por estaciones de bombeo, o también se puede tomar el conjunto entero de bombas independientemente de su ubicación o del tipo de bomba. En este caso, el sistema de ecuaciones sería:

$$
\boldsymbol{A} \boldsymbol{E}_{P}=\boldsymbol{W}_{P}
$$

donde $\boldsymbol{E}_{P}$ es nuevamente un vector columna con las densidades energéticas acumuladas para cada nudo y referidas al conjunto de bombas, y $\boldsymbol{W}_{P}$ es un vector columna que contiene la potencia de cada una de las bombas en la fila correspondiente al nudo aguas abajo de cada una de ellas. Según el principio de superposición, las soluciones del nuevo sistema global deben justificar la relación:

$$
e_{c}=\sum_{p \in P} e_{c, p}
$$


Si se aplican estas densidades energéticas globales a las ecuaciones (68) y (69) se obtiene la potencia de bombeo total transportada por cada tubería $W_{l}$, así como la potencia de bombeo total extraída en cada nudo de consumo $W_{c}$. La suma de este ultimo término para todos los nudos de consumo daría como resultado la potencia total aportada por las bombas.

\section{Fracciones de energía disipada}

Las pérdidas por fricción en tuberías y válvulas pueden manejarse bien individualmente, o bien agrupadas siguiendo el razonamiento anterior. La energía que se disipa en una línea en un instante de tiempo dado se expresa como $F_{l}=\gamma Q_{l} h_{l}$, siendo $Q_{l}$ el caudal que circula por la línea $l$ y $h_{l}$ la pérdida de carga correspondiente. Su transporte a través de la red puede interpretarse como la potencia que deja de transmitirse aguas abajo a causa de las pérdidas incurridas en la línea $l$. Del mismo modo, esta pérdida causará un déficit $f_{c, l}$ en la energía consumida en todos los nudos ubicados aguas abajo de dicha línea.

Para analizar la transmisión de dichas pérdidas se considerará, de manera análoga a la Ecuación (70), su densidad energética asociada que se define como:

$$
e_{c, l}=\frac{\sum_{\ell \in I_{c}} f_{\ell, l}}{\sum_{\ell \in I_{c}} Q_{\ell}}=\frac{\sum_{\ell \in I_{c}}\left(\tilde{e}_{\ell, l} Q_{\ell}+F_{\ell, l}\right)}{\sum_{\ell \in O_{c}} Q_{\ell}+Q_{c}}
$$

De manera análoga se define también el sistema de ecuaciones para la obtención de los valores de densidad energética:

$$
\boldsymbol{A} \boldsymbol{E}_{l}=\boldsymbol{F}_{l}
$$

donde $\boldsymbol{F}_{l}$ es un vector columna que contiene la pérdida de carga total de la línea $l$ en la fila correspondiente al nudo aguas abajo de dicha línea, y cero en el resto de las filas. Una vez se ha determinado la densidad energética en cada nudo, se puede calcular el déficit energético que se transporta a través de la línea $\ell$ debido a las pérdidas que se producen en la línea $l$ mediante una ecuación similar a la (68). Similarmente, mediante una ecuación como la (69) se puede calcular el déficit en la energía consumida en cada nudo debido a las pérdidas en la línea $l$, es decir, el valor de $f_{c, l}$ que aparece en la Ecuación (51) de balance de energía a nivel de nudo. 
También es posible construir un sistema matricial como el de la Ecuación (73) para determinar simultáneamente el efecto que producen conjuntamente todas las líneas del sistema, ya que el nivel de detalle al tratar cada línea independientemente resulta excesivo. El sistema matricial podría expresarse como:

$$
\boldsymbol{A} \boldsymbol{E}_{L}=\boldsymbol{F}_{L}
$$

donde $\boldsymbol{A}$ es la misma matriz que antes, $\boldsymbol{E}_{L}$ es ahora un vector columna que contiene las pérdidas acumuladas $e_{c, L}$ en cada nudo debidas al conjunto de los elementos disipativos ubicados aguas arriba, y $\boldsymbol{F}_{L}$ es un vector columna cuyos elementos corresponden a las pérdidas acumuladas de todas las tuberías con caudal entrante en cada nudo. Agrupar las pérdidas en este proceso facilita el cálculo del balance de energía a nivel de nudo, ya que las pérdidas acumuladas para todos los elementos disipativos de la red se obtienen directamente.

El proceso de simplificación de los cálculos puede ir más allá si se agrupan las pérdidas por fricción con la potencia de bombeo, y esto llevaría a determinar la energía neta que reciben los nudos de consumo. La ecuación de mezcla sería:

$$
e_{c, p l}=\frac{\sum_{\ell \in I_{c}}\left(\tilde{e}_{\ell, p l} Q_{\ell}+W_{\ell, p}-F_{\ell, l}\right)}{\sum_{\ell \in O_{c}} Q_{\ell}+Q_{c}}
$$

que llevaría a la resolución del sistema de ecuaciones:

$$
\boldsymbol{A} \boldsymbol{E}_{P L}=\boldsymbol{F}_{P L}
$$

donde el vector $\boldsymbol{F}_{P L}$ contendría el valor acumulado de la potencia de bombeo menos las pérdidas de todas las líneas aguas arriba del nudo en la correspondiente fila. La resolución del sistema arrojaría directamente los dos últimos términos de la parte izquierda en la Ecuación (51) de balance de energías.

\subsubsection{Comparación con los modelos de calidad del agua}

La Ecuación (78) se podría comparar con la ecuación de mezcla utilizada para el cálculo del transporte de una sustancia en un modelo de calidad del agua (Rossman, 2000). El parámetro equivalente a la densidad de energía $e_{c}$ en un modelo de calidad de agua sería la concentración de una sustancia en el nudo $c, W_{p}$ sería el equivalente al caudal másico de la sustancia inyectada en el nudo $p$, y las pérdidas por fricción $F_{l}$ serían comparables a la pérdida en el caudal másico de la sustancia a través de las tuberías a causa de su reacción en 
el medio o en la pared. Mientras que una sustancia puede ser o no ser reactiva, las pérdidas por fricción van a estar siempre presentes. El transporte exclusivo de la energía de bombeo, sin considerar las pérdidas, sería equivalente al transporte de una sustancia no reactiva.

Sin embargo, existe una diferencia esencial entre los dos modelos. El transporte de una sustancia es un proceso dinámico en el que la velocidad de transporte depende de la velocidad del flujo de agua. Por el contrario, el transporte de energía puede considerarse instantáneo a través de la red completa. Por lo tanto, no tiene sentido dividir las tuberías en segmentos, tal como lo hacen los modelos Eulerianos de transporte (Rossman et al., 1993), o hacer un seguimiento de los eventos de llegada de la sustancia a los nudos, tal como lo hacen los modelo Lagrangianos (Boulos et al., 1995). En este caso, la densidad energética en cada nudo para cada intervalo de tiempo tiene un único valor, y esto permite la introducción de los valores $\tilde{e}_{l, p}, \tilde{e}_{\ell, l}$ o $\tilde{e}_{\ell, p l}$, en referencia a las densidades de energía para el mismo intervalo de tiempo en los nudos aguas arriba de las correspondientes líneas. Es asimismo posible la construcción de un sistema lineal de ecuaciones cuya resolución proporciona los valores para las densidades de energía en cada nudo. En los modelos de calidad, puesto que la ecuación del transporte se resuelve mediante diferencias finitas, los valores de concentración en los nudos aguas arriba se calculan en un intervalo de tiempo previo, y las nuevas concentraciones se determinan a partir de estas, posteriormente a realizar la mezcla de las contribuciones másicas desde cada tubería de entrada. En este caso no es preciso resolver un sistema de ecuaciones, pero debe establecerse un valor inicial de concentración en cada nudo, a diferencia del caso del transporte de energía.

\subsubsection{Consideraciones adicionales}

Una conclusión interesante de la nueva formulación es que las presiones en los nudos pueden determinarse mediante el balance de energía expresado en la Ecuación (51), a condición de disponer de los caudales en todas las líneas, las alturas de impulsión en las bombas y las pérdidas de carga, como un método alternativo al procedimiento clásico basado en la altura piezométrica en los nudos. No obstante, el nuevo planteamiento aporta información acerca de la contribución de cada elemento de la red en la gestación de las presiones, es decir, el método permite determinar la parte de la presión observada en un nudo debida a la aportación energética de cada embalse, cada bomba y cada pérdida. 


\subsubsection{Ejemplo de aplicación}

En esta sección se presenta un ejemplo teórico con el objetivo de ilustrar los procesos descritos anteriormente. La Figura 39 muestra los elementos de la red de ejemplo y su distribución.

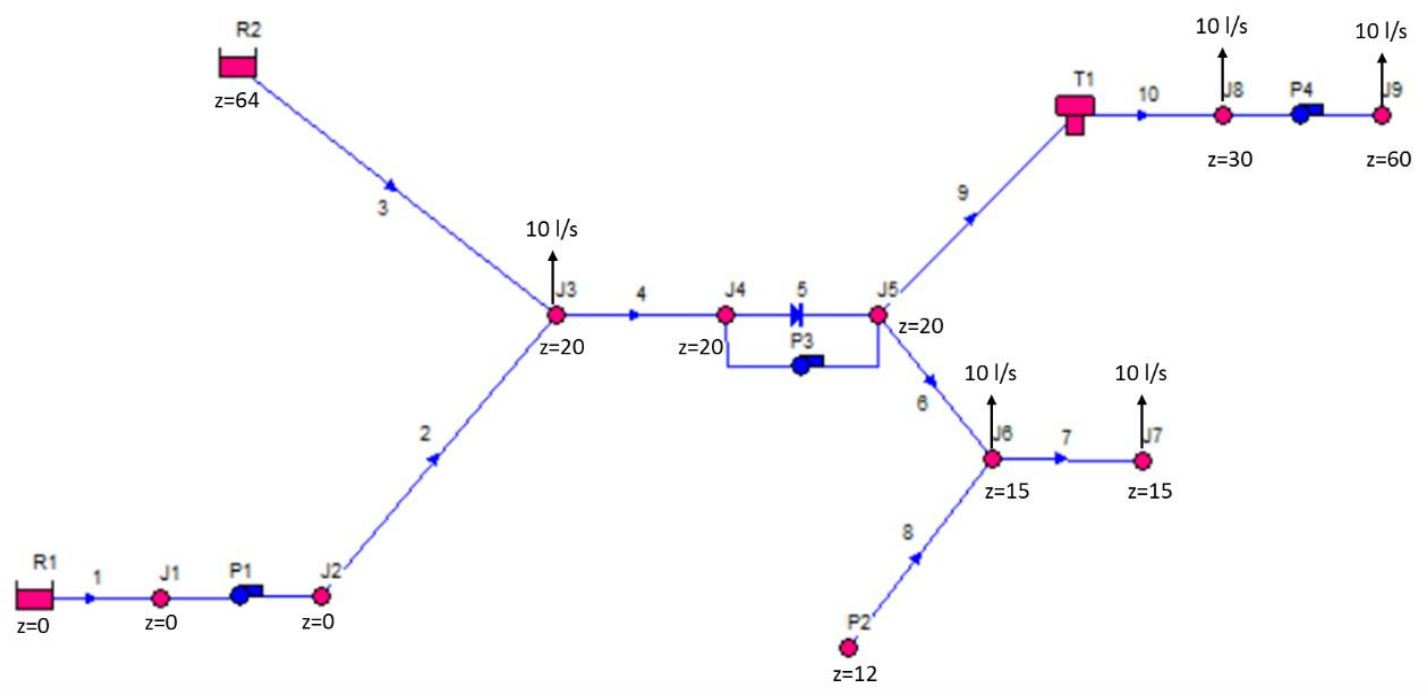

Figura 39. Red de ejemplo que incluye bombas de alimentación y sobrepresoras, embalses, nudos de demanda y un depósito con caudal entrante y saliente simultáneamente.

La red de estudio contiene dos embalses (R1 y R2) y un punto de inyección (P2), el cual se debe interpretar como una bomba de alimentación que ha sido modelada en EPANET como un nudo con una demanda negativa de 10 l/s. Estas tres fuentes de suministro entregan agua para abastecer a cinco demandas, ubicadas en los nudos J3, J6, J7, J8 y J9, cuyo valor es de 10 1/s en cada una de ellas (para el instante de estudio). La red contiene además un depósito (T1) de diámetro $15 \mathrm{~m}$ conectado por dos tuberías, lo que significa que puede combinar flujo de entrada y de salida simultáneamente. En tal caso, tal como se ha explicado, el depósito pasaría a considerarse como fuente de suministro y como punto de consumo a la vez. Finalmente, hay tres bombas ubicadas en distintos puntos de la red. Obsérvese que los embalses R1 y R2 son las dos principales entradas a la red. El primero está a una cota inferior y precisa de la bomba $\mathrm{P} 1$ para elevar suficientemente la presión para poder llegar a los nudos de consumo. El embalse R2 dispone de suficiente cota para alimentar al sistema por gravedad, pudiéndose ver esta característica como una oportunidad para la reducción del consumo energético. La introducción de la bomba de alimentación P2 (no modelizada directamente), puede justificarse para la satisfacción de una nueva demanda añadida en el nudo J7. La bomba P3 se incorpora para aumentar el volumen elevado hasta el depósito T1, 
mientras que la bomba P4 es necesaria para suministrar la suficiente presión al nudo de demanda J9, ubicada a la misma cota que la solera del depósito. Las bombas P1, P3 y P4 se han declarado de potencia constante, con $15 \mathrm{~kW}, 3 \mathrm{~kW}$ y $5 \mathrm{~kW}$ respectivamente.

Las cotas de los nudos y los embalses se muestran en la Figura 39. El nivel del depósito T1 se fija en $3 \mathrm{~m}$ para el instante de cálculo. Todas las tuberías tienen una longitud de 100 m, a excepción de las tuberías 2 y 9, cuya longitud es de 200 m, y las tuberías 5 y 10, que son de $10 \mathrm{~m}$. Para el cómputo de las pérdidas por fricción se ha tomado la ecuación de Darcy-Weisbach, suponiendo una rugosidad de 0,1 $\mathrm{mm}$ para todas las tuberías. Los caudales calculados mediante EPANET para el instante de tiempo analizado se muestran en la Figura 40.

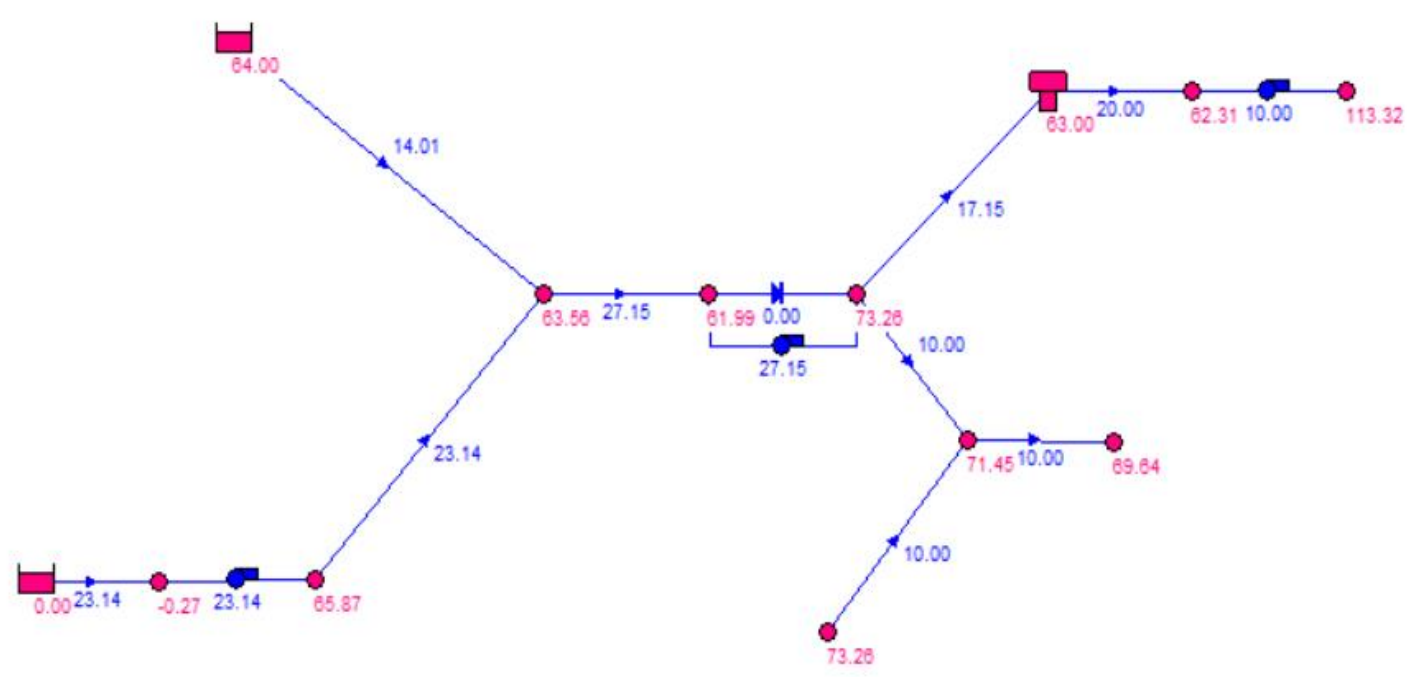

Figura 40. Alturas piezométricas y caudales para el instante de estudio.

En las Tablas Tabla 10 y Tabla 11 se resumen los resultados del balance de energías a nivel de nudo obtenidos para este ejemplo.

En la parte izquierda de la Tabla 10 se muestran las proporciones de caudal procedente de cada fuente de suministro $p_{c, s}$ en los nudos de consumo. Se comprueba la condición de que la suma de proporciones para un mismo nudo debe ser igual al $100 \%$. Análogamente, la suma por columnas de las proporciones multiplicadas por el caudal de salida coincide con el caudal total suministrado por la fuente. También se puede analizar cuál es el destino final del agua suministrada por cada fuente y en qué cantidades. 
En la parte derecha se muestran los valores de densidad energética en los nudos de consumo. La densidad energética de cada bomba se ha calculado independientemente, mientras que las de las pérdidas de carga se han agrupado en un único valor. Nótese que las densidades energéticas de un nudo se pueden combinar sumando las de las bombas y restando las de las pérdidas. Este valor indicaría la potencia total que realmente llega al nudo.

Tabla 10. Resultados en cada nudo de consumo de proporciones de caudal procedentes de cada fuente y densidades de energía relacionadas con las bombas y las pérdidas de carga.

\begin{tabular}{cccccccccc}
\hline Nudo & $\begin{array}{c}\boldsymbol{p}_{\boldsymbol{c}, \boldsymbol{R} 1} \\
(\boldsymbol{\%})\end{array}$ & $\begin{array}{c}\boldsymbol{p}_{\boldsymbol{c}, \boldsymbol{R} 2} \\
(\boldsymbol{\%})\end{array}$ & $\begin{array}{c}\boldsymbol{p}_{\boldsymbol{c}, \boldsymbol{P 2} 2} \\
(\boldsymbol{\%})\end{array}$ & $\begin{array}{c}\boldsymbol{p}_{\boldsymbol{c}, \boldsymbol{T 1 0}} \\
(\boldsymbol{\%})\end{array}$ & $\begin{array}{c}\boldsymbol{e}_{\boldsymbol{c}, \boldsymbol{P 1}} \\
\left(\mathbf{k W h} / \mathbf{m}^{3}\right)\end{array}$ & $\begin{array}{c}\boldsymbol{e}_{\boldsymbol{c}, \boldsymbol{P 2} 2} \\
\left(\mathbf{k W h} / \mathbf{m}^{3}\right)\end{array}$ & $\begin{array}{c}\boldsymbol{e}_{\boldsymbol{c}, \boldsymbol{P 3}} \\
\left(\mathbf{k W h} / \mathbf{m}^{3}\right)\end{array}$ & $\begin{array}{c}\boldsymbol{e}_{\boldsymbol{c}, \boldsymbol{P 4}} \\
\left(\mathbf{k W h} / \mathbf{m}^{3}\right)\end{array}$ & $\begin{array}{c}\boldsymbol{e}_{\boldsymbol{c}, \boldsymbol{L}} \\
\left(\mathbf{k W h} / \mathbf{m}^{3}\right)\end{array}$ \\
\hline J3 & 62.29 & 37.71 & 0 & 0 & 0.1123 & 0 & 0 & 0 & 0.0048 \\
J6 & 31.14 & 18.86 & 50.0 & 0 & 0.0561 & 0.0835 & 0.0154 & 0 & 0.0095 \\
J7 & 31.14 & 18.86 & 50.0 & 0 & 0.0561 & 0.0835 & 0.0154 & 0 & 0.0144 \\
T1 & 62.29 & 37.71 & 0 & 0 & 0.1123 & 0 & 0.0307 & 0 & 0.0371 \\
J8 & 0 & 0 & 0 & 100 & 0 & 0 & 0 & 0 & 0.0019 \\
J9 & 0 & 0 & 0 & 100 & 0 & 0 & 0 & 0.1390 & 0.0019 \\
\hline
\end{tabular}

Los resultados de la Tabla 11 se han extraído a partir de los anteriores. Cada término de potencia gravitatoria se calcula de acuerdo a la Ecuación (51), teniendo en cuenta el caudal procedente de cada fuente, y la diferencia de cota entre la fuente y el nudo de consumo. Las potencias de bombeo y las de pérdidas relacionadas con los caudales de demanda se calculan sencillamente multiplicando las densidades energéticas correspondientes por el caudal demandado. Siguiendo el balance a nivel de nudo propuesto, la potencia vinculada con la presión se puede obtener mediante la suma de las potencias aportadas (gravitatoria y de bombeo), menos la potencia perdida por fricción. Finalmente, la potencia anterior expresada en $\mathrm{kW}$ se puede transformar a m.c.a. dividiéndola por el caudal y por el peso específico del agua. Tal como se esperaba, la presión obtenida mediante este método coincide con la obtenida mediante el modelo hidráulico. Para los nudos cuya demanda es igual a cero, el balance se podría efectuar alternativamente en base al caudal total entrante al nudo, de modo que las potencias de bombeo, gravitatoria, y de pérdidas se han de referir a la potencia que entra en el nudo. Con este planteamiento, el balance determinaría la potencia asociada a la presión en el nudo igualmente. 
Tabla 11. Resultados del balance de energía a nivel de nudo en un instante dado para los nudos de demanda.

\begin{tabular}{ccccccccccccc}
\hline & \multicolumn{4}{c}{$\begin{array}{c}\text { Potencia gravitatoria } \\
\text { Nud) }\end{array}$} & \multicolumn{4}{c}{$\begin{array}{c}\text { Potencia de bombeo } \\
\text { (kW) }\end{array}$} & $\begin{array}{c}\text { Potencia } \\
\text { disipada } \\
\text { pérdidas } \\
(\mathbf{k W})\end{array}$ & $\begin{array}{c}\text { Potencia } \\
\text { útil como } \\
\text { presión } \\
(\mathbf{k W})\end{array}$ & $\begin{array}{c}\text { Presión } \\
(\mathbf{m})\end{array}$ \\
\cline { 2 - 13 } & $\mathrm{R} 1$ & $\mathrm{R} 2$ & $\mathrm{P} 2$ & $\mathrm{~T} 1$ & $\mathrm{P} 1$ & $\mathrm{P} 2$ & $\mathrm{P} 3$ & $\mathrm{P} 4$ & \\
\hline $\mathrm{J} 3$ & -1.22 & 1.63 & 0 & 0 & 4.04 & 0 & 0 & 0 & 0.17 & 4.27 & 43.56 \\
$\mathrm{~J} 6$ & -0.46 & 0.91 & $-0,15$ & 0 & 2.02 & 3.00 & 0.55 & 0 & 0.34 & 5.54 & 56.45 \\
$\mathrm{~J} 7$ & -0.46 & 0.91 & -0.15 & 0 & 2.02 & 3.00 & 0.55 & 0 & 0.52 & 5.36 & 54.64 \\
$\mathrm{~T} 1$ & -6.60 & 0.06 & 0 & 0 & 6.92 & 0 & 1.90 & 0 & 2.29 & 0 & 0 \\
$\mathrm{~J} 8$ & 0 & 0 & 0 & 3.24 & 0 & 0 & 0 & 0 & 0.07 & 3.17 & 32.31 \\
$\mathrm{~J} 9$ & 0 & 0 & 0 & 0.29 & 0 & 0 & 0 & 5.00 & 0.07 & 5.23 & 53.32 \\
\hline & & & & & & & & & & & \\
Total & $\mathbf{- 8 . 7 4}$ & $\mathbf{3 . 5 0}$ & $\mathbf{- 0 . 2 9}$ & $\mathbf{3 . 5 3}$ & $\mathbf{1 5 . 0 0}$ & $\mathbf{6 . 0 0}$ & $\mathbf{3 . 0 0}$ & $\mathbf{5 . 0 0}$ & $\mathbf{3 . 4 6}$ & $\mathbf{2 3 . 5 7}$ & \\
\hline
\end{tabular}

Debe remarcarse que el caudal total que entra en la red es de $47,15 \mathrm{l} / \mathrm{s}$, mientras que la demanda total es de 50 1/s. La diferencia es aportada por el depósito T1. Además, la potencia total gravitatoria aportada por los embalses y el depósito es de $-2,0 \mathrm{~kW}$. Esto significa que el sistema no podría abastecerse únicamente por gravedad. La potencia total aportada por las bombas es de 29,02 kW y la potencia perdida por fricción es de 3,46 kW, lo que arroja un balance neto de entrada al sistema de 25,57 kW. Añadiendo la potencia gravitatoria a esta cifra, resulta una potencia de $23,57 \mathrm{~kW}$. El mismo balance se puede aplicar a cada nudo individualmente.

Finalmente, se puede ver que la potencia total que llega a cada nudo de demanda procedente de las bombas menos la potencia de pérdidas es menor que la potencia asociada a la presión. Esto significa que, gracias a la mayor cota de las fuentes de suministro con respecto a los nudos de demanda, hay una cierta cantidad de potencia hidráulica disponible para compensar las pérdidas por fricción o para elevar la presión en estos nudos. Por otro lado, la suma de potencia gravitatoria que se entrega al depósito T1 es negativa. Esto significa que parte de la energía consumida por las bombas está destinada a superar la diferencia de cota entre las fuentes y el depósito.

\subsubsection{Conclusiones}

El balance propuesto en esta sección finalmente trasciende las limitaciones de los términos de energía dependientes del datum de la red, que han sido ampliamente utilizados hasta ahora en muchos balances energéticos. Este nuevo enfoque permitirá la definición de indicadores de desempeño más significativos y coherentes para su uso en la gestión de sistemas de abastecimiento de agua. 
Se ha propuesto un nuevo balance energético a nivel de nudo para un instante dado. El objetivo último de este enfoque es la adquisición de información útil para la operación en tiempo real de sistemas de distribución de agua, aunque los resultados del balance también se podrían utilizar para identificar debilidades estructurales relacionadas con los rendimientos energéticos de la red y, por lo tanto, sería de utilidad para definir mejoras estratégicas. El balance propuesto proporciona información sobre cómo la energía de cualquier fuente, por ejemplo, una bomba o depósito en particular, se distribuye entre los diferentes elementos de un sistema de distribución de agua, permitiendo, en última instancia, calcular el coste económico del agua entregada a cada nudo.

El método propuesto puede mostrar si la potencia gravitatoria contribuye a satisfacer una demanda particular o si se requiere potencia de bombeo adicional para vencer las diferencias de cota. También se puede determinar qué fracción del coste energético se ha utilizado para compensar las pérdidas por fricción, qué fracción en aumentar la presión en los nudos de consumo y, si procede, la fracción utilizada para superar las diferencias de cota.

Este estudio también ha definido un procedimiento sistemático y general para calcular los términos del balance de energía, que puede ser fácilmente implementado en cualquier plataforma informática.

El siguiente paso en el desarrollo de este método pasa por determinar las ecuaciones para un período de simulación extendido, lo que permitiría a los operadores supervisar y controlar el coste variable del agua almacenada y, por lo tanto, tomar decisiones informadas a corto plazo para mejorar la eficiencia energética de sus redes.

\subsection{Metodología de optimización basada en el coste del agua almacenada}

El balance de energía detallado que se ha propuesto en la sección anterior establece las bases a partir de las cuales se espera que se puedan desarrollar nuevos indicadores de desempeño, nuevos métodos para evaluar las ineficiencias o debilidades de una red a nivel estratégico o nuevos métodos para la optimización de la operación diaria.

La presente sección pretende ilustrar las posibilidades del nuevo enfoque a través de un sencillo ejemplo teórico que ofrece una alternativa a los métodos de optimización en tiempo real que se han abordado en la Tesis. Todos los métodos planteados trabajan a partir de un horizonte temporal determinado, estableciendo como resultado las consignas de 
operación óptimas que deberán ejecutarse a futuro. La alternativa que se propone aquí, por el contrario, trata de tomar buenas decisiones apoyándose únicamente en información del pasado y del presente.

Tal como se puede intuir, este tipo de estrategia conducirá a soluciones subóptimas. Sin embargo, debe tenerse en cuenta que ninguno de los métodos de optimización puede garantizar que se alcance el óptimo global en el problema de minimización del consumo energético de redes hidráulicas ya que, en principio, únicamente la programación lineal y la cuadrática garantizan este punto y, en estos casos, deben realizarse simplificaciones de las ecuaciones hidráulicas introduciendo así un error que, en mayor o menor medida, alejarán las soluciones del óptimo global. Además, estos enfoques pueden requerir la inclusión de ciertas predicciones, cuyo error distorsiona también los resultados. Entre estas predicciones, la más importante es la predicción de la demanda de agua, pero, a medida que aumente la penetración de las energías renovables en los sistemas de distribución de agua, otro tipo de predicciones, como la radiación solar, temperatura o la velocidad y dirección del viento, cobrarán especial relevancia en el ámbito de la optimización energética.

Así pues, se puede asumir que en la optimización de la operación de la red se trabaja en general con soluciones subóptimas. En estas condiciones, se valora el estudio de métodos de optimización voraces, capaces de ofrecer buenas soluciones, aunque subóptimas, pero con la gran ventaja de requerir un esfuerzo computacional mínimo.

\subsubsection{Equiparación de la densidad de energía a la densidad de coste}

Se puede relacionar la potencia hidráulica con la potencia eléctrica consumida $P_{p}$ a través del rendimiento global $\eta_{p}$, que dependerá del punto de funcionamiento en cada instante.

$$
P_{p}=\frac{\gamma Q_{p} \Delta H_{p}}{\eta_{p}}=\frac{W_{p}}{\eta_{p}}
$$

La potencia eléctrica consumida no podría formar parte del balance de energía de la Ecuación (51), pero nada impide que se pueda aplicar el mismo método de transporte, basado en el reparto proporcional a los caudales de línea, con el fin de obtener en cada nudo de consumo la cantidad de electricidad que se ha requerido para satisfacer su demanda. 
De manera similar, puede relacionarse la potencia eléctrica consumida con el coste incurrido a través de la aplicación del término de energía de la tarifa eléctrica $C e(t)$.

$$
C_{p}=P_{p} \operatorname{Ce}(t)
$$

Análogamente al planteamiento del balance de energía a nivel de nudo, se define $C_{c}$ como la fracción del coste en energía incurrido por las bombas que llega al punto de consumo $c, \mathrm{y} c_{c, p}$ es la fracción del coste incurrido por la bomba $p$ y que llega al punto $c$, de modo que:

$$
\sum_{p \in P} c_{c, p}=C_{c}
$$

Para el cálculo de los términos $c_{c, p}$ del sistema, se utilizará el concepto de densidad de coste. Tal como se ha definido en el apartado 5.2.1.3, la densidad de energía $e_{p}$ responde a la cantidad de energía por unidad de volumen que se ha transportado hasta un determinado punto desde una fuente de energía. Así, el flujo que atraviesa una bomba $p$ adquiere una densidad de energía $e_{p}=W_{p} / Q_{p}\left(\mathrm{kWh} / \mathrm{m}^{3}\right)$. Esta densidad hace referencia a la potencia hidráulica, ya que debía responder a su aportación al balance energético.

De modo paralelo a la densidad de energía, se puede definir una nueva variable intensiva que responda a la cantidad de unidades monetarias por unidad de volumen que es transportada desde las fuentes de energía que incurren en un coste energético hasta los puntos de consumo. El flujo que trasiega una bomba adquiere una densidad de coste:

$$
\xi_{p}=\frac{W_{p} C e(t)}{Q_{p} \eta_{p}}=\frac{P_{p} C e(t)}{Q_{p}}
$$

En este caso, se utilizará también una densidad distinta para cada nudo, cuya expresión es:

$$
\xi_{c, p}=\frac{\sum_{l \in I_{c}} c_{l, p}}{\sum_{l \in I_{c}} Q_{l}}
$$

donde el numerador responde al coste económico total procedente de la bomba $p$ que llega al nudo $c$ en un determinado instante, y el denominador indica el caudal total que entra en dicho nudo. La expresión que determina la fracción de coste que es transportado por una tubería será: 


$$
c_{l, p}=\tilde{\xi}_{l, p} Q_{l}
$$

siendo $\tilde{\xi}_{l, p}$ la variable nodal asociada al nudo aguas arriba de la línea $l$ y que coincide con la variable referente a líneas $\xi_{l, p}$.

Los costes asociados a los consumos externos se determinan como:

$$
c_{c, p}=\xi_{c, p} Q_{c}
$$

donde el valor de esta nueva variable en cada nudo de consumo puede calcularse de acuerdo a la siguiente expresión:

$$
\xi_{c, p}=\frac{\sum_{l \in I_{c}}\left(\tilde{\xi}_{l, p} Q_{l}+C_{l, p}\right)}{\sum_{l \in O_{c}} Q_{l}+Q_{c}}
$$

donde $\tilde{\xi}_{l, p}$ es la variable nodal que indica la densidad de coste del nudo aguas arriba de la línea $l$, y $C_{l, p}$ es coste en energía incurrido por la bomba $p$, el cual valdrá cero para todas las líneas excepto para aquellas que representan una bomba. La Ecuación (87) planteada para todos los nudos del sistema, conduce a un sistema lineal de ecuaciones que puede expresarse matricialmente como:

$$
A \xi_{p}=C_{p}
$$

donde $\boldsymbol{A}$ es la misma matriz definida en la sección anterior, $\boldsymbol{\xi}_{p}$ es el vector de incógnitas que identificará las densidades de coste referentes a la bomba $p$ en todos los nudos del sistema, y $\boldsymbol{C}_{p}$ es un vector que contiene el coste en energía que se ha incurrido por la bomba $p$ en la fila que identifica al nudo aguas abajo de la misma bomba, siendo cero el resto de los elementos del vector.

\subsubsection{Función de mezcla en los depósitos}

Hasta ahora, el balance de energía se ha planteado para un único instante. Para poder extender el balance a dos o más instantes de cálculo consecutivos debe resolverse el problema de tratamiento de los depósitos. Se trata de un problema complejo que debe tener en cuenta la distinta naturaleza de la energía gravitatoria y de la energía de bombeo, que obligará a distinguir los procesos mediante los cuales el depósito almacena uno u otro tipo de energía. 
El trabajo completo se desarrollará en el futuro, limitándose este apartado de la Tesis a describir el proceso de mezcla de la energía que aportan las bombas. El motivo principal para priorizar este estudio reside en el hecho de que la energía gravitatoria es una energía natural, ligada a la orografía del terreno, que no tiene coste económico. La única energía que realmente tiene coste es la consumida en los bombeos. Por ello, en primer lugar, se tratará de proponer el método de cálculo del coste asociado a una determinada energía de presión, o al agua almacenada en un depósito.

El hecho de que en el balance propuesto en la sección anterior se diferencie la energía de bombeo de la energía disipada en el transporte y seguimiento de estas a través de la red, obedece precisamente al interés por calcular posteriormente el coste energético, pues a los mismos resultados se habría llegado si aportes y pérdidas de energía se hubieran tratado conjuntamente. Esta diferenciación es particularmente importante en el caso de los depósitos intermedios. La energía de presión en ellos es cero, y toda la energía aportada, ya sea gravitatoria o de bombeo, es compensada por la energía perdida en rozamientos. Sin embargo, al analizarlas por separado, es posible calcular el coste del agua almacenada en un depósito, como el coste de la energía de bombeo consumida para hacer llegar ese volumen de agua hasta el depósito, aunque dicha energía se haya disipado después en pérdidas por el camino.

Para realizar el seguimiento de la variable asociada al coste en energía en un periodo extendido se va a suponer que en los depósitos se realiza una mezcla completa entre el coste $(€)$ almacenado, más el coste del agua que entra menos el del agua que sale.

La ecuación de mezcla en los depósitos se diferencia de la Ecuación (87) en que, además de contemplar el balance entre las entradas y salidas, debe incorporar el intervalo de tiempo $\Delta t$ y el volumen almacenado $\mathrm{V}_{d}$ como variables adicionales:

$$
\xi_{c, p}^{t+1}=\frac{\sum_{l \in I_{c}}\left(\tilde{\xi}_{l, p} Q_{l}+C_{l, p}\right)-\xi_{c, p}^{t}\left[\sum_{l \in O_{c}} Q_{l}+Q_{c}-\mathrm{V}_{d} /_{\Delta t}\right]}{\sum_{l \in I_{c}} Q_{l}-\left[\sum_{l \in O_{c}} Q_{l}+Q_{c}-\mathrm{V}_{d} / \Delta t\right]}
$$

El flujo saliente del depósito en cada instante se considerará que tiene la densidad de coste media del agua almacenada en el mismo en el instante anterior a la mezcla, $\xi_{c, p}^{t}$.

La presencia de depósitos en el sistema crea un problema de cálculo, y es la determinación del valor inicial de la densidad energética en estos, la cual será desconocida. 
La solución consistirá, tal como se hace en los modelos de calidad, en asumir un valor inicial, por ejemplo, cero, y ciclar el sistema repitiendo el periodo de cálculo muchas veces. En cada ciclo el valor inicial se iguala al valor final del ciclo anterior, hasta que finalmente este se estabilice.

Obsérvese que, a diferencia de los modelos de calidad, no se requiere establecer una densidad de coste inicial en cada nudo, pues al considerarse la transmisión de energía (y, por tanto, de coste) instantánea, el valor estable de dicha densidad se obtiene por cálculo directo, mediante la metodología expuesta anteriormente.

Si se considerara un modelo de mezcla FIFO o LIFO en los depósitos, entonces sería posible determinar la hora en que entró en el depósito el caudal que se está consumiendo actualmente y, por tanto, su coste o periodo tarifario. Para aplicar este concepto habría que definir previamente los intervalos horarios que diferencian cada fracción de volumen. Cada vez que transcurre dicho tiempo se crea una nueva fracción de volumen, identificado por el intervalo horario. Dicha fracción se consumirá la primera o la última según el modelo sea LIFO o FIFO respectivamente. Cada fracción de volumen se podrá a su vez compartimentar por la fuente de suministro o la fuente de energía. Pero estos análisis se realizarán ya en trabajos futuros.

\subsubsection{Algoritmo voraz de optimización}

Gracias a los resultados derivados de la idea de que la energía se transporta a lo largo de la red siguiendo los caudales de línea, se puede inferir con exactitud el coste energético que tiene el agua almacenada en los depósitos en cada instante. Con esta valiosa información, se pueden tomar decisiones de operación en tiempo real evaluando las posibles acciones sobre la red y su influencia sobre el coste del agua en los depósitos.

El método que se propone aquí se presenta como una alternativa al método lineal propuesto en el CAPÍTULO 4. Así, el ámbito de aplicación coincide, siendo de interés en subsistemas de bombeo para redes de abastecimiento de agua, en cuya regulación participen bombas, depósitos y válvulas de regulación.

Las restricciones y variables son idénticas al problema detallado en el CAPÍTULO 4, pero en este caso no se trabaja sobre un horizonte temporal extendido, sino que se resuelve 
un problema de optimización para cada instante de tiempo ${ }^{5}$. Además, aquí la variable es discreta, no continua, y en este ejemplo se utilizará por sencillez la variable binaria.

El objetivo que se busca es mantener la densidad de coste del agua almacenada en los depósitos del subsistema en el menor valor posible. Se ha de tener en cuenta que en subsistemas con más de un depósito, al existir un valor de densidad distinto para cada uno de ellos, es preciso determinar un único indicador que represente al conjunto. A tal fin, se propone la utilización de la densidad de coste promedio $\xi^{t}$ ponderada por el volumen almacenado:

$$
\bar{\xi}^{t}=\frac{\sum_{d=1}^{N d} \sum_{p=1}^{N p} \xi_{d, p}^{t} V_{d}^{t}}{\sum_{d=1}^{N d} V_{d}^{t}}
$$

donde $N d$ es el número de depósitos del sistema y $N p$ el número de bombas, mientras que $\xi_{d, p}^{t}$ es la densidad de coste en el instante $t$ y $V_{d}^{t}$ el volumen total del depósito $d$.

El valor de $\bar{\xi}^{t}$ dependerá de su estado anterior $\bar{\xi}^{t-1}$ y de la estrategia de control elegida en el instante $t-1$. Por lo tanto, para minimizar la densidad de coste promedio se evalúa en un instante la consecuencia de cada posible acción de control. Para que ello resulte factible, es indispensable que el subsistema a optimizar contenga pocos elementos de control, ya que la enumeración de todas las posibles acciones resulta en un problema combinatorio cuyo espacio de soluciones crece exponencialmente con el número de variables. Alternativamente, se podría reducir el espacio de soluciones limitando el número de combinaciones a un conjunto más pequeño. Por ejemplo, en una estación de bombeo con varias bombas idénticas en paralelo se podría determinar una prioridad de arranque, de manera que no todas las combinaciones fueran permitidas, con lo que bastaría determinar el número de bombas que deben estar activas ${ }^{6}$. También, si la reducción en las combinaciones permitidas no fuese suficiente, se podría combinar el método con un algoritmo evolutivo.

\footnotetext{
${ }^{5}$ La filosofía de esta forma de proceder coincide con la que se propone en la mejora del algoritmo de maximización del volumen a entregar por gravedad. Se busca la máxima simplificación del proceso de optimización para lograr que el tiempo de computación sea lo suficientemente bajo como para poder utilizar los algoritmos en la toma de decisiones en tiempo real.

${ }^{6}$ En una estación con 10 bombas, el espacio de soluciones pasaría de contener $2^{10}$ alternativas a solamente 11.
} 
Así, se exploraría el espacio de soluciones sin la evaluación exhaustiva de todas las combinaciones.

Nótese que si en un determinado instante $t-1$ se opta por una solución en la que todas las bombas del subsistema están paradas, consecuentemente el valor de $\bar{\xi}^{t}$ en el instante posterior no podrá incrementarse. Así, siempre existe una solución que, si no mejora, al menos mantiene la densidad de coste promedio. Esto permitiría que una estrategia de optimización basada en la elección en cada instante de la combinación de control que minimice la densidad de coste promedio llevaría a una trayectoria de este parámetro en el tiempo decreciente. Sin embargo, con toda probabilidad los depósitos tenderían a vaciarse, obligando a efectuar bombeos en instantes que no serían idóneos y posiblemente penalizarían considerablemente el coste total.

Por ello, la estrategia de optimización heurística que se propone precisa la distinción de dos subconjuntos de variables. El subconjunto $X^{+}$contiene todas las combinaciones de variables en las que al menos una bomba está activa. El subconjunto $X^{0}$ contiene el resto de las combinaciones (todas las bombas están paradas). Así, se propone el siguiente planteamiento para la optimización en cada instante:

$$
\begin{array}{ll}
\min \bar{\xi}^{t}\left(X^{+}\right) & \text {si } \bar{\xi}^{t}\left(X^{+}\right) \leq \bar{\xi}^{t-1}+\varepsilon \\
\min \bar{\xi}^{t}\left(X^{0}\right) & \text { si } \bar{\xi}^{t}\left(X^{+}\right)>\bar{\xi}^{t-1}+\varepsilon
\end{array}
$$

donde $\varepsilon$ es un parámetro configurable, gracias al cual se permitirán soluciones que empeoren de manera limitada la densidad de coste promedio anterior, siempre que alguna bomba esté en funcionamiento.

\subsection{Resultados}

El algoritmo voraz propuesto en este capítulo se ha aplicado al mismo caso de estudio presentado en el apartado 4.4 con ánimo de realizar una comparativa entre este y el método de optimización basado en programación lineal. Aunque la ejecución del algoritmo voraz de optimización se realiza para un único instante, se ha iterado hasta completar las 24 horas correspondientes al escenario de optimización lineal.

Tal como se ha comentado, la metodología de cálculo de los flujos energéticos requiere el establecimiento de la densidad de coste (o energía) inicial en los depósitos. Para el caso de estudio se han realizado varias iteraciones imponiendo sucesivamente como 
densidad de coste inicial la que se obtiene como resultado de la optimización, finalmente convergiendo a una densidad de coste inicial de $0.015 € / \mathrm{m}^{3}$. En cuanto al parámetro configurable $\varepsilon$, se ha optado por establecer un valor de $0.0015 € / \mathrm{m}^{3}$. Cuanto mayor es este parámetro, mayor es la tendencia a elevar el nivel del agua en los depósitos y viceversa.

La ejecución de los 24 pasos del algoritmo ha supuesto un tiempo de computación de $857 \mathrm{~ms}$ con un procesador Intel Core i5 de $8^{\mathrm{a}}$ generación. Los caudales y niveles que resultan de la aplicación del algoritmo voraz se muestran en las siguientes figuras.

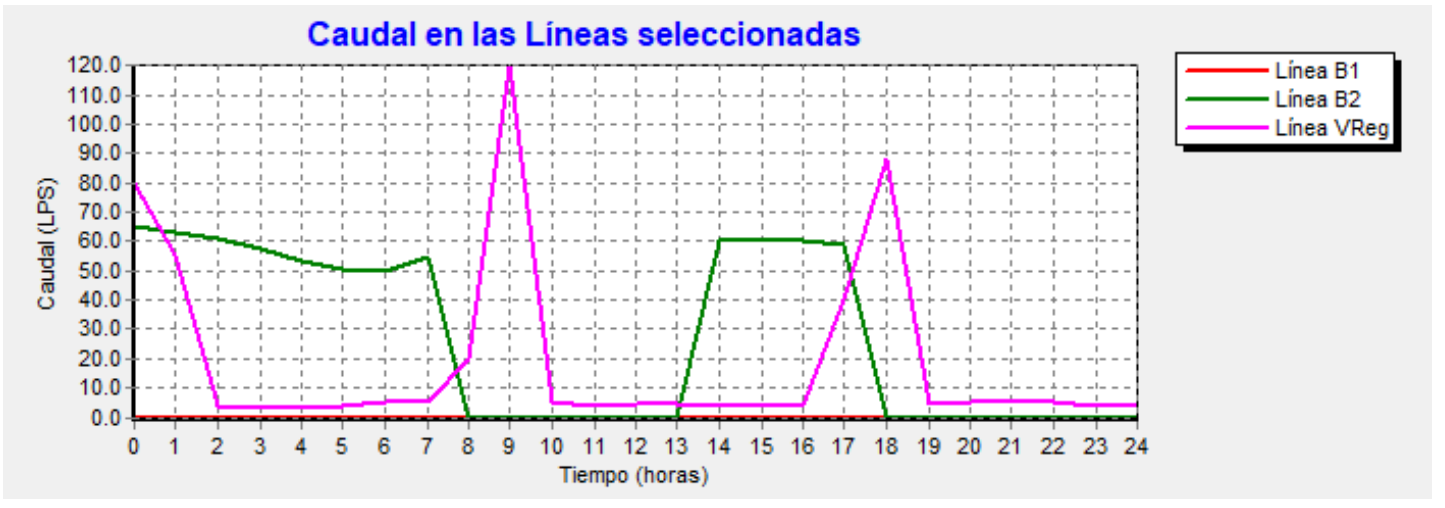

Figura 41. Caudal trasegado por las bombas B1 y B2, y por la válvula VReg en el escenario optimizado mediante el algoritmo voraz.

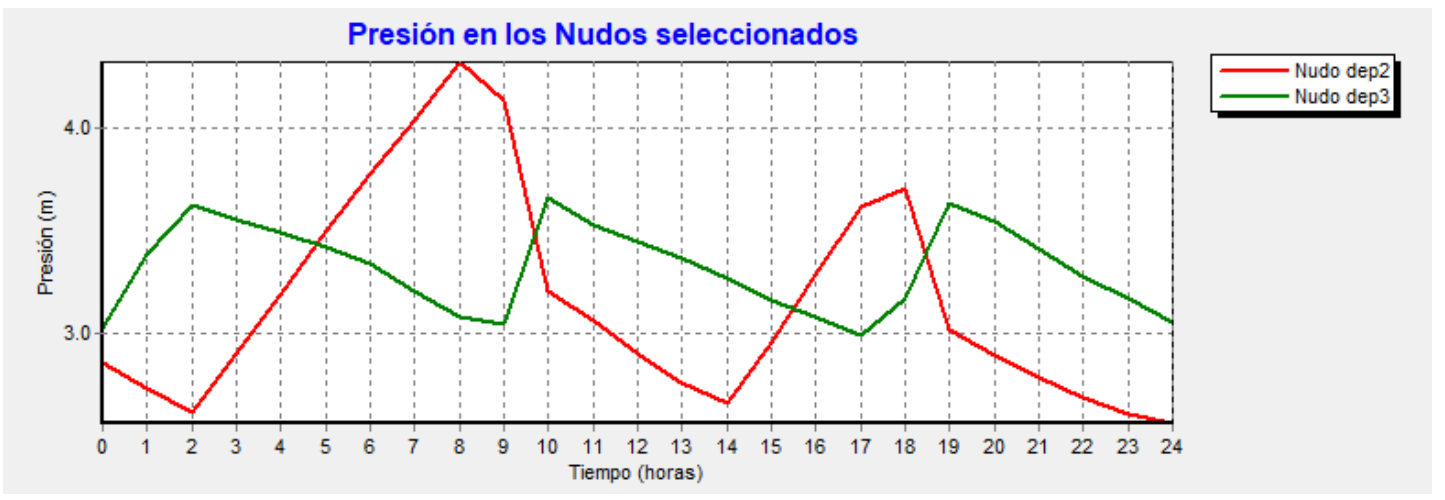

Figura 42. Evolución del nivel en los depósitos dep2 y dep3 en el escenario optimizado mediante el método voraz.

En la Tabla 12 se resumen los principales indicadores que permiten la comparación entre el escenario inicial, obtenido mediante la simulación de las consignas de operación que en realidad se ejecutaron el día 1-6-21, el escenario optimizado mediante el método lineal descrito en el apartado 4.2, y el escenario optimizado mediante el algoritmo voraz planteado en este apartado. 
Mediante el método lineal se obtiene una mejora porcentual del $8,85 \%$ en el coste específico respecto al escenario inicial. Mediante el algoritmo voraz se obtiene una mejora del 7,62\%, pudiéndose considerar que alcanza una efectividad comparable al método lineal, teniendo en cuenta que, aunque el método lineal incluye como restricción que el nivel final de los depósitos sea mayor o igual al nivel inicial, se puede observar en los resultados que, a causa del error cometido en la linealización del modelo, esta restricción no se cumple. Al bombear un volumen total menor, se tiene mayor disposición de horas en las que la energía es más barata y, por tanto, se pueden alcanzar mejores resultados, a costa de dejar los niveles más bajos para el día siguiente.

Tabla 12. Resumen comparativo entre los resultados de la simulación de la operación real, el escenario propuesto por el optimizador lineal y el escenario propuesto por el algoritmo voraz.

\begin{tabular}{llll}
\hline & Escenario inicial & Método lineal & Algoritmo voraz \\
\hline Volumen bombeado $\left(\mathrm{m}^{3}\right)$ & $2.494,92$ & $2.426,7$ & $2.499,69$ \\
Energía consumida $(\mathrm{kWh})$ & 129,02 & 129,61 & 136,07 \\
Coste energía $(€)$ & 12,43 & 11,02 & 11,51 \\
Coste específico $\left(\mathrm{c} € / \mathrm{m}^{3}\right)$ & 0,4983 & 0,4542 & 0,4604 \\
\hline
\end{tabular}

\subsection{Conclusiones}

El novedoso método para el cálculo de los flujos energéticos en los SDA que se ha propuesto en este capítulo permite en última instancia conocer y monitorizar el coste incurrido para transportar el agua desde las fuentes hasta los depósitos de regulación y, finalmente, hasta los puntos de consumo. Con esta valiosa información, se ha propuesto un sencillo método basado en una heurística que observa el coste del agua almacenada en los depósitos y toma las decisiones de operación en función de las variaciones de este.

Una de las principales características del método heurístico propuesto es la voracidad, ya que es capaz de tomar las decisiones tomando datos de un único instante de tiempo. Esta característica hace que, a priori, el método sea idóneo para la optimización en tiempo real porque, potencialmente, requiere un menor esfuerzo computacional y porque evita la necesidad de realizar predicciones para las variables futuras.

El método heurístico se ha comparado con el método basado en la optimización lineal descrito en el capítulo anterior, aplicándolo a la resolución del mismo caso de estudio, y 
alcanzando un resultado similar en cuanto a ahorros alcanzados y en cuanto a esfuerzo computacional.

Las ventajas esperables del método heurístico respecto al método lineal estarían relacionadas con dos aspectos, el uso del modelo hidráulico completo, y la independencia de predicciones futuras.

Hay que tener en cuenta que en el caso de estudio resuelto mediante programación lineal el error cometido en la simplificación del modelo es reducido, con lo que la solución ofrecida no se aleja en gran medida de la óptima. Además, se ha asumido que la demanda es conocida y, por tanto, no hay error en la predicción.

En los sistemas de transporte cuyo comportamiento sea más marcadamente no lineal, el método heurístico, al trabajar sobre el modelo hidráulico completo, sería capaz de calcular los distintos puntos de funcionamiento de las bombas, evitando aquellos en los que el rendimiento se vea penalizado. Este aspecto diferenciador respecto del método lineal supone, además, que se busque indirectamente una reducción en el consumo de energía, no únicamente en el coste. El uso del modelo hidráulico completo también evita que el error desplace la solución propuesta de la óptima, como ocurre en el método lineal.

En cuanto a la dependencia de las predicciones, en la operación en tiempo real, el cálculo de la estrategia óptima únicamente sería posible con una predicción perfecta de las variables en el futuro. Entre estas variables se encuentra el coste de la energía y la demanda de los usuarios. En el mercado eléctrico español, el coste de la electricidad hora a hora para el día posterior es conocido a partir de las 12:00 del mediodía, con lo que en este caso no habría incertidumbre. En la predicción de las demandas, por el contrario, sí que existe un pequeño grado de incertidumbre, de modo que la variación en el consumo real respecto a la predicción también causaría una desviación del óptimo en los métodos tradicionales, aunque en este caso se trata de pequeñas desviaciones.

Finalmente, aunque no se aborda en la presente Tesis, dada la creciente necesidad de aumentar la sostenibilidad de los sistemas consumidores de energía, se está propiciando la incorporación de sistemas de generación eléctrica renovable en puntos estratégicos donde se pueda aprovechar directamente esta energía limpia y las estaciones de bombeo son buenas candidatas para la introducción de energías renovables como la fotovoltaica, ya que, además de suponer el consumo energético más importante en los sistemas de distribución de agua, 
es probable que en las inmediaciones de la instalación exista un depósito de regulación cuya parte superior proporcione la superficie necesaria para la ubicación de los paneles solares.

El método heurístico de optimización que se plantea en este capítulo se adaptaría perfectamente a las instalaciones de autoconsumo sin vertido a red y sin almacenamiento, en las cuales la energía que no se consume en el momento de producirse es desaprovechada. En tiempo real, se analizarían los posibles escenarios de operación teniendo en cuenta la energía renovable disponible, además del precio de la energía procedente de la red eléctrica en ese momento.

En resumen, los métodos de optimización voraces, basados en buenas heurísticas, pueden suponer una alternativa útil a los métodos tradicionales de optimización en el ámbito de la optimización en tiempo real de los parámetros de operación en sistemas de distribución de agua. 


\section{CAPÍtULO 6.}

\section{TRATAMIENTO DE LOS DATOS DE CAMPO}

Para concluir las aportaciones realizadas en esta Tesis se ha incluido este capítulo adicional sobre el tratamiento de los datos de campo pues, aunque no está vinculado directamente con los algoritmos de optimización energética y su voracidad, aborda un aspecto fundamental para el éxito en la implantación práctica en el mundo real de las técnicas propuestas.

Un algoritmo de optimización en tiempo real trabaja sobre datos reales, y la fiabilidad de los datos tomados en campo es fundamental para garantizar los resultados obtenidos por el optimizador. Tratándose de un proceso en tiempo real, no hay opción para depurar los datos en diferido, días después de haber sido tomados. Los datos de campo deben depurarse también en tiempo real, y el presente capítulo se ha destinado a tratar algunas técnicas propuestas para ello.

\subsection{Introducción}

Para poder realizar la carga de un modelo hidráulico es imprescindible que todos los datos a partir de los cuales se calculan las demandas y las leyes de control estén completos y sean coherentes. Dada la gran cantidad de señales que se requieren, normalmente no se dispondrá de esta calidad en los datos brutos, ya que pueden darse múltiples casuísticas por las que no se disponga de algún dato en un instante determinado (fallos en sensores, comunicaciones, almacenamiento, procesos de transformación, etc.) (Hutton et al., 2014).

Por ello, es indispensable que la carga del modelo se realice posteriormente a un proceso de filtrado de datos que garantice, en la medida de lo posible, la calidad de todos los datos (Mair et al., 2014; Rodriguez et al., 2017; Seo y Gary M. Marsh, 2006). En 
consecuencia, se ha diseñado y desarrollado un procedimiento para el filtrado capaz de, por un lado, detectar los datos que probablemente sean erróneos y, por otro lado, restituir dichos datos con valores más razonables. El procedimiento se apoya en una serie de metodologías que se describen a continuación.

\subsection{Detección y clasificación de los datos anómalos}

La detección de los datos anómalos siempre debe ser un paso previo a su restitución. En la práctica conviene distinguir entre una anomalía grave y otra leve. Mientras que los datos anómalos leves pueden mantenerse para posteriores análisis y calificarse únicamente como valores 'extraños', los graves deben ser necesariamente restituidos para evitar errores en los cálculos o en las conclusiones extraídas de los datos.

Como después se verá, una técnica para restituir los datos anómalos graves es el uso de patrones. Puesto que los patrones se confeccionan a partir de los datos históricos, aquellos que haya sido calificados como datos anómalos, tanto leves como graves, no deberían utilizarse en la confección y mantenimiento de dichos patrones.

La ausencia de datos (DBNULL) es la causa más evidente de la necesidad de restitución de valores, además de, probablemente, la más común. Además de esta casuística, también se pueden considerar erróneos los valores de una serie atendiendo a su valor. De modo general, se han definido cinco criterios a observar para este marcado. Se podría considerar un dato erróneo si:

- $\quad$ Es mayor a un determinado umbral (VAL_H) (anómalo leve)

- Es mayor a un determinado umbral extremo (VAL_HH) (anómalo grave)

- Es menor a un determinado umbral (VAL_L) (anómalo leve)

- Es menor a un determinado umbral extremo (VAL_LL) (anómalo grave)

- Es cero (NULL), y no se permiten valores nulos (por ejemplo, un caudal podría ser nulo perfectamente, pero no un nivel o una presión, salvo circunstancias excepcionales).

- La señal es plana (FLAT). Es decir, todos los instantes tiene el mismo valor, y el sistema está activo.

- El valor de la señal excede en más de 1,5 veces (anómalo leve) o en más de 3 veces (anómalo grave) el rango intercuartílico por encima del tercer cuartil o por debajo del primer cuartil ('outliers' o valores atípicos), esto es, si: 
- $q<Q_{1}-1,5$ (ó 3) $I Q R$ ó $q>Q_{3}+1,5$ (ó 3) $I Q R$, con $I Q R=Q_{3}-Q_{1}$

- Una señal marcada como errónea por alguno de los criterios anteriores se utiliza para calcular otras variables derivadas. En este caso, estas también se marcarán como erróneas (DEPENDENT)

Los umbrales VAL_HH y VAL_LL pueden configurarse bien particularizados para cada variable, o bien utilizando un criterio común para todo un conjunto de señales (presiones, caudales, etc.). Por su parte, NULL y FLAT también se configuran para cada señal, ya que muchas de ellas sí que pueden permitir estas casuísticas. A la hora de adoptar un coeficiente de exclusión para los outliers, este puede hacerse depender también de cada variable, teniendo en cuenta su irregularidad.

En cuanto a las dependencias, sirva el siguiente ejemplo para justificar el criterio. Se tiene una variable A calculada como la suma de otras dos variables B y C.

$$
A=B+C
$$

B y C, en principio se observan como correctas por no incumplir ninguno de los cuatro criterios anteriores. Sin embargo, la suma de estas (A) sí que llega a rebasar algún umbral. En este caso, al no saber a priori cuál de las dos variables B o C es la causa del error, se deben marcar ambas como erróneas.

En caso de que B o C no tuviesen valor (DBNULL), entonces A tampoco tendría valor y, en consecuencia, sería marcada como errónea. Sin embargo, esto supone una excepción en el criterio de marcado DEPENDENT, puesto que, en este caso, evidentemente, el error es causado por la o las variables que no tienen valor. Por tanto, si alguna de ellas sí que tiene valor y no ha sido marcada como errónea, se conservará como correcta.

\subsection{Metodología de restitución de los datos anómalos}

Solo los valores marcados como anómalos graves deberían ser restituidos, al menos a efectos de cálculo o análisis posteriores, y aunque sea de forma temporal. Caso de almacenarlos de forma permanente deberá hacerse sobre una serie copia de la original, de modo que la primera nunca se pierda, dejando constancia del cambio. Además, los valores restituidos nunca deben utilizarse para actualizar los patrones empleados en la propia restitución, si fuera el caso, para evitar el viciado de estos. 
Admitiremos en este punto que de cada variable de campo a consultar se dispone de su patrón o comportamiento esperado para un determinado periodo de tiempo. La generación de patrones es un campo de estudio con un gran interés en el ámbito de la investigación hoy en día, debido en parte al auge de las técnicas de inteligencia artificial. El alcance de la presente Tesis no incluye nuevas investigaciones en este sentido.

Los patrones utilizados en este apartado han sido obtenidos sencillamente a partir del cálculo de las medianas de las series de datos observados correspondientes a una misma hora de diferentes días. Estos patrones son de granularidad horaria (24 datos por día). Los valores del patrón se utilizan para la restitución de los datos erróneos de una serie, según distintas casuísticas que se han contemplado y que se detallan a continuación.

\subsubsection{Restitución de datos erróneos a partir de patrones}

Admitiremos en este apartado que de cada variable de campo a consultar se dispone de su patrón o comportamiento esperado para un determinado periodo de tiempo. Dichos patrones se les supone por ahora de granularidad horaria (24 datos por día), y se propone obtenerlos simplemente a partir de las medianas de los valores medios horarios observados a lo largo de un cierto periodo del pasado reciente. El hecho de utilizar las medianas evita que los valores extremos puedan distorsionar el valor típico esperado de la variable en cada una de las horas abarcadas por el patrón.

Si la granularidad del patrón no fuera horaria, se puede convertir fácilmente a cualquier otra granularidad, como se describe en el apartado siguiente. Así pues, vamos a formular a continuación el proceso de restitución de los valores anómalos para el caso más general.

Se tiene una serie de datos $S=\left\{s_{0}, s_{1}, \ldots, s_{n-1}\right)$ con un total de $n$ elementos, con granularidad $g \in\{5,15,60\}$, en la que el instante inicial coincide con la hora del día $h_{\text {ini }}(S) \in\{0,1,2, \ldots, 23\}$. Dicha serie contiene una subserie $E=\left\{s_{i}, s_{i+1}, \ldots, s_{m-1}\right) \in S$ de $m-i$ elementos, tal que $0 \leq i<m \leq n$, siendo además todos sus elementos consecutivos.

Se tiene por otra parte, el patrón $P$ asociado a la serie, que deberá tener la misma granularidad que la serie $S$. Teniendo en cuenta que la función $\operatorname{ind}(x, S)$ devolvería el índice que ocupa el elemento $x$ dentro de la serie $S$, que $S(i)$ expresa el elemento i-ésimo de la 
serie y que la función $\operatorname{Res}(x / y)$ devuelve el residuo del cociente, se definen las desviaciones inicial y final para el ajuste de $E(S)$ sobre $P$ como:

$$
\begin{aligned}
& \delta_{\text {ini }}=S(\operatorname{ind}[E(0), S]-1)-P\left(\operatorname{Res}\left[\frac{\operatorname{ind}[E(0), S]-1+\frac{60}{g} \cdot h_{\text {ini }}(S)}{1440 / g}\right]\right) \text { si ind }[E(0), S]=0
\end{aligned}
$$

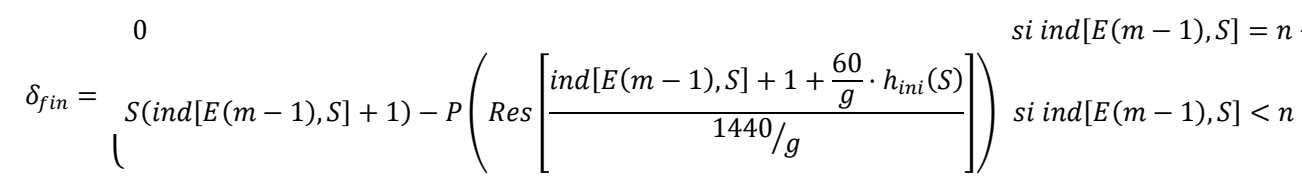

Una vez definidas estas desviaciones de la serie a filtrar respecto del patrón, se procede a sustituir los valores de $E$ por el siguiente ajuste del patrón:

$$
E(j)=P\left(\operatorname{Res}\left[\frac{\operatorname{ind}[E(j), S]+\frac{60}{g} \cdot h_{i n i}(S)}{1440 / g}\right]\right)+\frac{j+1}{m-i+2} \cdot \delta_{f i n}+\frac{m-i-j+1}{m-i+2} \cdot \delta_{i n i}
$$

El objetivo final de estas expresiones es conseguir intercalar los valores del patrón en sustitución de la serie de datos anómalos, manteniendo la continuidad por los extremos con los datos válidos y adecuando los valores del patrón para tener una transición suave entre dichos extremos.

\subsubsection{Combinación de distintas granularidades en las series de datos}

Como se ha comentado, la granularidad de los patrones es siempre horaria, pero los datos de campo pueden estar disponibles con granularidades menores, como por ejemplo cada quince minutos o cada cinco minutos. En estos casos hay que realizar un proceso previo de adaptación del patrón horario original $P_{o}$ a la granularidad correspondiente $\left(P_{15 m}, P_{5 m}\right)$ por simple repetición de los valores correspondientes a cada hora:

$$
\begin{gathered}
P_{o}=\left\{p_{t 0}, p_{t 1}, p_{t 2}, \ldots, p_{t 23}\right\} \\
P_{15 m}=\left\{p_{t 0}, p_{t 0}, p_{t 0}, p_{t 0}, p_{t 1}, p_{t 1}, p_{t 1}, p_{t 1}, \ldots, p_{t 23}, p_{t 23}, p_{t 23}, p_{t 23}\right\}
\end{gathered}
$$


siempre que la pauta de las medidas sea inferior a la del patrón. En caso contrario, menos frecuente, de que la pauta de las medidas sea mayor que la del patrón, bastará agrupar y promediar los valores del patrón para adecuarlos a la nueva pauta.

En cualquier caso, se admitirá que, si la pauta de las medidas no coincide con la del patrón, la primera será divisor o múltiplo de la segunda.

\subsubsection{Caso particular. Ajuste de las medidas de los caudalímetros en base al balance caudales}

La restitución de datos faltantes o erróneos por el método de ajuste a patrón descrito anteriormente puede, en ocasiones, no llegar a conseguir resultados razonables cuando las distintas variables están interrelacionadas entre sí. Es el caso particular de que las variables se correspondan con medidas de los caudales entrantes a un sector de demanda, cuyo balance neto debe estar acorde con la propia demanda del sector.

Por ello, se ha tratado de alcanzar la coherencia de los datos planteando, para los instantes en que se da algún valor erróneo, un problema de optimización mediante un balance de caudales basado en la definición de los sectores de inyección.

Cada sector de inyección se maneja independientemente. Las variables involucradas en cada problema de optimización son los caudales que entran en juego en el balance del sector, así como, en caso de tratarse de caudales calculados, las variables (caudales o niveles) a partir de los cuales se calculan.

Sirva como ejemplo un sector de inyección cuyo caudal inyectado se calcula como la suma de $Q_{I 1}$ y $Q_{I 2}$, y que contiene en su interior dos sectores de demanda, cuyo caudal demandado viene definido por $Q_{D 1}$ y $Q_{D 2}$ respectivamente.

$$
\begin{aligned}
Q_{I 1} & =\left\{q_{I 1}^{0}, q_{I 1}^{1}, q_{I 1}^{2}, q_{I 1}^{3}\right\} \\
Q_{I 2} & =\left\{q_{I 2}^{0}, q_{I 2}^{1}, q_{I 2}^{2}, q_{I 2}^{3}\right\} \\
Q_{D 1} & =\left\{q_{D 1}^{0}, q_{D 1}^{1}, q_{D 1}^{2}, q_{D 1}^{3}\right\} \\
Q_{D 2} & =\left\{q_{D 2}^{0}, q_{D 2}^{1}, q_{D 2}^{2}, q_{D 2}^{3}\right\}
\end{aligned}
$$


Si un caudal (o más) estuviese marcado como erróneo para un instante dado (por ejemplo: $q_{I 1}^{1}$ es erróneo), se habrá sustituido su valor por el del patrón ajustado $p_{I 1}^{1}$ tal como se ha descrito en el apartado anterior, de modo que se tendría:

$$
Q_{I 1}=\left\{q_{I 1}^{0}, p_{I 1}^{1}, q_{I 1}^{2}, q_{I 1}^{3}\right\}
$$

Entonces, para el instante $\mathrm{t}=1$ se puede plantear el siguiente problema de minimización:

$$
\begin{aligned}
\min f(X)=F_{b} & \left(x_{I 1}^{1}+x_{I 2}^{1}-x_{D 1}^{1}-x_{D 2}^{1}\right)^{2}+F_{I 1}\left(x_{I 1}^{1}-p_{I 1}^{1}\right)^{2} \\
& +F_{I 2}\left(x_{I 2}^{1}-q_{I 2}^{1}\right)^{2}+F_{D 1}\left(x_{D 1}^{1}-q_{D 1}^{1}\right)^{2}+F_{D 2}\left(x_{D 2}^{1}-q_{D 2}^{1}\right)^{2}
\end{aligned}
$$

donde el vector de variables $X$ alberga los nuevos valores para todos los caudalímetros, tanto los que han sido marcados como erróneos como los que no. El primer término calcula el balance del sector tratando de igualar el caudal inyectado con el demandado, y se pondera por un factor $F_{b}$. Los cuatro siguientes términos tratan de minimizar la diferencia entre las nuevas variables y el valor de caudal medido o del patrón ajustado en su caso, cada una de ellas ponderada con su correspondiente factor.

En principio el método no tiene en cuenta que las variables no marcadas como erróneas no deberían modificarse. Sin embargo, ello puede forzarse dando mucho más peso a los factores que multiplican a los términos correspondientes a las variables aceptadas que a los que multipliquen a los términos de las variables marcadas como erróneas.

El problema planteado puede resolverse mediante un algoritmo de programación cuadrática, que es un proceso para minimizar una función cuadrática con restricciones lineales y cuya expresión formal es:

$$
\begin{gathered}
\min f(x)=x^{T} Q x+c^{T} x \\
\text { s. } a . \quad A x \leq b
\end{gathered}
$$

En el paquete de .NET Accord se puede encontrar un algoritmo apropiado para ello.

Para poder utilizar la programación cuadrática debe expresarse el problema en la forma indicada anteriormente, es decir, deben calcularse los coeficientes de la matriz $Q$ y del vector $c$. Si se desarrollan los cuadrados, por una parte, y se realizan a continuación las multiplicaciones matriciales, se llega a las siguientes identidades, donde la matriz $Q$ resulta ser simétrica:

$$
Q_{i, i}=F_{b}+F_{i}
$$




$$
\begin{gathered}
Q_{i, j}=Q_{j, i}=\operatorname{sgn}(i, j) \cdot F_{b} \\
c_{i}=-2 \cdot F_{i} q_{i}
\end{gathered}
$$

Se tiene que $\operatorname{sgn}(i, j)=-1$ si $i$ es un caudal de un sector de inyección y $j$ de demanda, y $\operatorname{sgn}(i, j)=1$ en caso contrario. Además, $\operatorname{sgn}(i, j)$ se verá afectado con un factor adicional de -1 en caso de que algún caudal sea de salida del correspondiente sector.

\subsubsection{Caso de que algún caudal sea calculado}

Si uno de los caudales que forman parte del balance es una variable calculada a partir de otros caudales o de niveles de depósito, estas señales se incorporarán como nuevas variables al problema de optimización. Siguiendo el ejemplo anterior, supongamos que $Q_{D 1}$ se calcula a partir de la suma de dos caudales $Q_{D 1_{-} A}+Q_{D 1_{-} B}$, entonces el problema de minimización se completaría del siguiente modo:

$$
\begin{gathered}
\min f(X)=F_{b}\left(x_{I 1}^{1}+x_{I 2}^{1}-x_{D 1}^{1}-x_{D 2}^{1}\right)^{2}+F_{I 1}\left(x_{I 1}^{1}-p_{I 1}^{1}\right)^{2} \\
+F_{I 2}\left(x_{I 2}^{1}-q_{I 2}^{1}\right)^{2}+F_{D 1}\left(x_{D 1}^{1}-q_{D 1}^{1}\right)^{2}+F_{D 2}\left(x_{D 2}^{1}-q_{D 2}^{1}\right)^{2} \\
+F_{D 1 \_A}\left(x_{D 1 \_}^{1}-q_{D 1 \_A}^{1}\right)^{2}+F_{D 1 \_B}\left(x_{D 1 \_B}^{1}-q_{D 1 \_B}^{1}\right)^{2} \\
\text { s. } a_{\cdot}: \quad x_{D 1}^{1}-x_{D 1}^{1}-x_{D 1}^{1}=0
\end{gathered}
$$

El término correspondiente al balance no se ha modificado, sin embargo, se han añadido dos términos a la función de minimización para ajustar el resultado de las nuevas variables a su valor real (o patrón en caso de ser erróneos) y, además, se ha añadido una restricción lineal de igualdad para forzar a que el caudal calculado siga respetando su fórmula.

Si algún caudal es calculado a partir de la variación de nivel de un depósito, el planteamiento se complica. Hasta ahora el balance se ha planteado para un solo instante de tiempo. Pero si, póngase como ejemplo, $Q_{I 1}$ se calculase a partir de la variación de nivel del depósito $N_{D E P}$, esto implicaría que entraría en juego el valor de una nueva variable en otro instante de tiempo.

La solución que se ha adoptado ha sido la de generalizar en estos casos el problema para múltiples instantes, simplemente repitiendo la estructura matricial descrita tantas veces como instantes incluya la optimización. De este modo, el planteamiento para 2 instantes, añadiendo los términos involucrados en el cálculo $Q_{I 1}$ a partir de niveles, sería como sigue: 


$$
\begin{aligned}
\min f(X)=F_{b} & \left(x_{I 1}^{1}+x_{I 2}^{1}-x_{D 1}^{1}-x_{D 2}^{1}\right)^{2}+F_{I 1}\left(x_{I 1}^{1}-p_{I 1}^{1}\right)^{2} \\
& +F_{I 2}\left(x_{I 2}^{1}-q_{I 2}^{1}\right)^{2}+F_{D 1}\left(x_{D 1}^{1}-q_{D 1}^{1}\right)^{2}+F_{D 2}\left(x_{D 2}^{1}-q_{D 2}^{1}\right)^{2} \\
& +F_{D 1 \_}\left(x_{D 1 \_}^{1}-q_{D 1 \_}^{1}\right)^{2}+F_{D 1 \_B}\left(x_{D 1 \_B}^{1}-q_{D 1 \_B}^{1}\right)^{2} \\
& +F_{D E P}\left(x_{D E P}^{1}-n_{D E P}^{1}\right)^{2}+F_{b}\left(x_{I 1}^{2}+x_{I 2}^{2}-x_{D 1}^{2}-x_{D 2}^{2}\right)^{2} \\
& +F_{I 1}\left(x_{I 1}^{2}-p_{I 1}^{2}\right)^{2}+F_{I 2}\left(x_{I 2}^{2}-q_{I 2}^{2}\right)^{2}+F_{D 1}\left(x_{D 1}^{2}-q_{D 1}^{2}\right)^{2} \\
& +F_{D 2}\left(x_{D 2}^{2}-q_{D 2}^{2}\right)^{2}+F_{D 1 \_A}\left(x_{D 1 \_A}^{2}-q_{D 1 \_A}^{2}\right)^{2} \\
& +F_{D 1 \_B}\left(x_{D 1 \_B}^{2}-q_{D 1 \_B}^{2}\right)^{2}+F_{D E P}\left(x_{D E P}^{2}-n_{D E P}^{2}\right)^{2} \\
& \quad \begin{array}{c}
x_{D 1}^{1}-x_{D 1}^{1}-x_{D 1}^{1}=0 \\
x_{I 1}^{1}-\left(x_{D E P}^{1}+x_{D E P}^{2}\right) \cdot \frac{A_{D E P}}{\Delta t}=0
\end{array}
\end{aligned}
$$

Para decidir cuántos instantes se van a incluir en el problema de optimización se observarán únicamente las variables de nivel de depósito. Dado que la restitución de un nivel en un instante de tiempo $t$ afectaría tanto al caudal calculado del instante anterior como al instante $\mathrm{t}$, se han de enlazar necesariamente los instantes t-1 y t siempre que se dé un error en un nivel en el instante t. Además, como se ha de incluir necesariamente el nivel en el instante $t+1$ como variable para poder calcular la restricción de variable calculada a partir de niveles, también se debe enlazar el instante $t+1$. En resumen, un único error en una variable de nivel forzaría a enlazar tres instantes de tiempo en el problema de optimización.

En caso de que en el instante $t+1$ también hubiese error en el nivel se enlazaría el siguiente instante $(\mathrm{t}+2)$ y así sucesivamente, pudiendo llegar a encadenar todos los instantes de simulación en el mismo problema.

Finalmente, se indican los factores que se han utilizado al aplicar este planteamiento a un caso particular:

$$
F_{b}=200
$$

Para el cálculo del resto de factores, que acompañan a cada variable independientemente, se han tenido en cuenta las siguientes casuísticas:

- Si la medida es errónea, directamente $F_{x}=0.01$

- Si no es errónea se evalúan las siguientes características: 
- Se define $f_{\text {niv }}$, de modo que si se trata de un nivel $f_{\text {niv }}=10^{9}$, en caso contrario $f_{\text {niv }}=10$

- Se define $f_{\text {com }}$, de modo que si la variable aparece en más de un sector de inyección $f_{\text {com }}=100$, en caso contrario $f_{\text {com }}=1$

- Se define $f_{\text {iny, }}$, de modo que si es un caudalímetro que interviene en la inyección de caudal al sector $f_{\text {iny }}=1$, en caso contrario $f_{\text {iny }}=0$

- El factor $F_{x}$, en caso de acompañar a un dato correcto tendrá el siguiente valor:

$$
F_{x}=f_{\text {com }}\left[f_{\text {niv }}\left(1+f_{\text {iny }}\right)\right]
$$




\section{CAPÍtULO 7.}

\section{CONCLUSIONES}

\subsection{Conclusiones generales}

A lo largo del periodo de tiempo en el que se ha desarrollado la presente Tesis se han explorado las herramientas y metodologías disponibles para la optimización energética en tiempo real de los sistemas de distribución de agua a presión. Se ha adquirido un valioso conocimiento general sobre modelización hidráulica y la problemática derivada de la conexión de los modelos con los datos de campo en tiempo real. El objetivo final del trabajo de modelización en el contexto de la Tesis es el de explotar estos modelos, capaces de reproducir fielmente la realidad, en la simulación de nuevos escenarios de operación en los que se reduzca el consumo de energía externa necesaria para la satisfacción de las demandas.

Se ha abordado de manera aislada la optimización energética de los subsistemas de transporte de agua y la de los subsistemas de distribución (riego por inyección directa) debido a las distintas características que se pueden observar en ellos.

En el trabajo con los subsistemas de distribución se han explorado las posibilidades que ofrecen los métodos metaheurísticos de optimización, realizando varias aportaciones originales orientadas a la mejora en la eficiencia computacional de los mismos debido a la necesidad de obtener una respuesta más rápida que sea compatible con la toma de decisiones en tiempo real.

En concreto, se ha demostrado mediante el análisis de una serie de casos que un planteamiento multiobjetivo permite alcanzar la convergencia en un número menor de evaluaciones, hasta un 50\% menos para alcanzar el mismo resultado, y que la paralelización de dicho cálculo utilizando procesadores multi-core consigue reducir aproximadamente un 
$80 \%$ el tiempo de computación. Además, para el caso de estudio, se ha conseguido una reducción del coste en energía del 6-7\% con ausencia de déficits de presión en las tomas activas.

Se ha desarrollado también una metodología para la obtención de una programación de riego que maximiza el volumen total entregado sin aporte de energía en un determinado intervalo de tiempo, a la vez que garantiza una presión mínima en las tomas activas. Con esta metodología se han obtenido incrementos en el volumen entregado por gravedad del $30 \%$ aproximadamente, presentando presiones de trabajo mayores que la deseada en cada instante. Además, se ha logrado reducir el tiempo de cálculo respecto a la metodología de partida.

En cuanto a los subsistemas de transporte, se ha explorado la aplicabilidad del método determinista de optimización por programación lineal a la vista de las importantes ventajas que presenta respecto al resto de métodos generales de optimización.

En concreto, el método se ha aplicado con éxito a un caso de estudio real obteniendo reducciones en el coste específico diario del orden del 10\%. La eficiencia computacional ha quedado evidenciada, con un tiempo de computación inferior a 1 segundo para el caso de estudio descrito.

Asimismo, en el contexto de los subsistemas de transporte, se ha trabajado en la definición de una buena heurística basada en el cálculo del coste energético y/o económico del agua entregada en los puntos de consumo y en los depósitos intermedios, que ha permitido formular un algoritmo voraz para la optimización energética en cada instante de tiempo. Este método ha conseguido igualar el desempeño alcanzado mediante la programación lineal y se espera que ofrezca unas mejores capacidades en sistemas con un comportamiento más marcadamente no lineal, así como también una mejor adaptación a problemas de optimización con participación de energías renovables.

Finalmente, el desarrollo de la presente Tesis ha propiciado la oportunidad de trabajar en la optimización de sistemas reales. Como conclusión derivada indirectamente de la participación en los pilotos reales, cabe mencionar que la implantación efectiva de aplicaciones que, mediante los métodos propuestos, sirvan como sistemas de soporte a la decisión a los gestores de las redes hidráulicas precisa de una gran confianza por parte de estos gestores tanto en la precisión de los modelos hidráulicos construidos, como en los 
propios resultados de la optimización. A día de hoy, todavía existe una cierta desconfianza que causa una ralentización en la implantación efectiva de este tipo de sistemas de soporte a la decisión, pero se espera que en pocos años se produzca una aceptación generalizada y se normalice entre las empresas y comunidades de usuarios la explotación de herramientas que ayuden a una mejor toma de decisiones en la operación diaria de los sistemas de distribución de agua.

\subsection{Aportaciones originales}

Las principales aportaciones al campo del conocimiento en la operación de sistemas de distribución de agua y que se recopilan en este documento son las siguientes:

- Mejora del método general de optimización de redes de riego (apartado 3.3).

- Mejora del método de maximización del volumen entregado por gravedad (apartado 3.4).

- Propuesta de procedimiento sistemático para la construcción de las ecuaciones del método lineal de optimización (apartado 4.2)

- Método de cálculo de los flujos energéticos en un SDA (apartado 5.2)

- Método heurístico de optimización de sistemas de transporte de agua (apartado $5.3)$.

- Ajuste de los datos filtrados en base a los balances de caudales (apartado 6.3.3).

\subsection{Publicaciones}

\subsubsection{Revistas JCR}

Alonso Campos, J.C., Jiménez-Bello, M.A., Martínez Alzamora, F. 2020. “Real-Time

Energy Optimization of Irrigation Scheduling by Parallel Multi-Objective Genetic

Algorithms." Agricultural Water Management 227.

doi:10.1016/j.agwat.2019.105857. Q1

Conejos, P., Martínez Alzamora, F., Hervás Carot, M., Alonso Campos, J.C. 2020.

"Building and Exploiting a Digital Twin for the Management of Drinking Water

Distribution Networks." Urban Water Journal 17 (8). Taylor \& Francis: 704-713.

doi:10.1080/1573062X.2020.1771382. Q2 
Jiménez-Bello, M. A., Alonso Campos, J.C., Manzano-Juárez, J., Martínez Alzamora, F. 2021. "Methodology for Flushing Pressurised Irrigation Networks for Fertigation and Operation Maintenance Purposes.” Irrigation Science 39 (3): 375-384. doi:10.1007/s00271-021-00724-4. Q1

\subsubsection{Congresos}

Alonso Campos, J.C., Jiménez Bello, M.A., Martínez Alzamora, F. 2017. “Optimización Energética En Tiempo Real de La Programación Del Riego Mediante Algoritmos Genéticos Multi-Objetivo Paralelos," V Jornadas de Ingeniería del Agua (JIA 2017), 51-52. A Coruña. ISBN: 978-84-9749-670-4

Alonso Campos, J.C., Jiménez-Bello, M.A., Martínez Alzamora, F., Bou Soler, V. 2015. "Nueva Metodología Para Optimizar La Programación Del Riego Aprovechando La Diferencia de Cota Entre El Punto de Suministro y El de Consumo.” XXXIII Congreso Nacional de Riegos, 450-459. Valencia. doi:http://dx.doi.org/10.4995/CNRiegos.2015.1518.

Conejos, P., Martínez Alzamora, F., Alonso Campos, J.C. 2017. “A Water Distribution System Model to Simulate Critical Scenarios by Considering Both Leakage and Pressure Dependent Demands." Procedia Engineering 186: 380-387. doi:10.1016/j.proeng.2017.03.234.

\subsubsection{Libros}

Vegas Niño, O.T., Martínez Alzamora, F., Alonso Campos, J.C., Tzatchkov, V.G. 2017. Iniciación a La Programación Con La Toolkit de Epanet v2.00.12 En Un Entorno Windows. 1st ed. Jiutepec, México: Instituto Mexicano de Tecnología del Agua. http://repositorio.imta.mx/handle/20.500.12013/2076

Vegas Niño, O.T., Martínez Alzamora, F., Alonso Campos, J.C., Tzatchkov, V.G. 2018. Using the Epanet Toolkit v2.00.12 with Different Programming Environments. 1st ed. Jiutepec, México: Instituto Mexicano de Tecnología del Agua. http://repositorio.imta.mx/handle/20.500.12013/2187 


\subsection{Discusión y limitación de resultados}

Tal como se ha descrito en los correspondientes capítulos, las metodologías propuestas para la optimización energética de redes de agua se apoyan en modelos hidráulicos. La construcción y correcta calibración de un modelo hidráulico es una tarea compleja que presenta una fuerte dependencia con la disponibilidad y calidad de los datos procedentes de las distintas fuentes de información.

La búsqueda del escenario óptimo se realiza a través de la simulación de escenarios no observados mediante el modelo hidráulico de la red. Por ello, lógicamente, si el modelo hidráulico no reproduce fielmente el comportamiento real de la red, los resultados a los que conducirán los algoritmos de optimización no serán correctos. Este aspecto supone una limitación a la hora de aplicar las metodologías propuestas, ya que una correcta construcción y calibración de los modelos requiere un importante esfuerzo previo que muchas veces implicará, no solo el trabajo de modelado, sino también una inversión en la instalación de sensores adicionales.

Así pues, se requiere una evaluación previa de cada caso en la que se estimen los potenciales ahorros y se cotejen con la inversión necesaria en la instalación de instrumentación adicional y en el trabajo de construcción de un modelo adecuado.

En esta línea, otra limitación de los resultados obtenidos es precisamente el orden de magnitud de las mejoras alcanzadas. Para el caso de los subsistemas de transporte, en los casos de estudio en los que se ha trabajado, los porcentajes de reducción de los costes han sido del orden del 5 - 10\%. Lógicamente, el grado de mejora depende de cada caso y de la situación de partida. Pero, teniendo en cuenta que este porcentaje de mejora no se refiere a la totalidad de la factura eléctrica, sino únicamente al término de energía, puede ocurrir que en subsistemas con un coste energético no muy elevado no compense la adición de complejidad a los automatismos de control dados los escasos ahorros potenciales en términos absolutos.

Por otra parte, las predicciones utilizadas para la construcción de los escenarios futuros juegan un papel fundamental en los resultados de las simulaciones y, por tanto, de los algoritmos de optimización. Así pues, el error cometido en estas predicciones puede conllevar una reducción en el porcentaje de mejora real frente al calculado una vez se lleven a cabo las consignas de operación propuestas. Aunque se puede considerar que las demandas 
urbanas de agua potable son muy estables y que, por lo tanto, las predicciones a corto plazo (24 horas) tienen poco error, esto podría cambiar en sectores con pocos usuarios o con presencia de otros tipos de consumidores (industriales, riegos, etc.).

En cuanto a los propios resultados que se obtienen mediante las distintas metodologías, tal como ya se ha apuntado, no existe garantía de que se ha alcanzado el óptimo global, así como tampoco se puede conocer con certeza cuán lejos está del óptimo global la solución propuesta. Aunque este hecho supone una limitación de las metodologías, a la vista de los ahorros obtenidos se asume que la mejora es suficiente, teniendo en cuenta el compromiso existente entre calidad de la solución y tiempo de computación.

En consecuencia, en el desarrollo de esta Tesis se han concentrado los esfuerzos, no tanto en la búsqueda de unos mayores ahorros energéticos, sino en tratar de aumentar la aplicabilidad de las técnicas de optimización en sistemas reales gracias a una reducción de los tiempos de computación necesarios, haciéndolas así compatibles con la toma de decisiones en la operación diaria de las redes de agua.

\subsection{Trabajos futuros}

En cada línea de trabajo englobada en el desarrollo de la presente Tesis se han vislumbrado nuevas acciones con las que profundizar y mejorar de algún modo las metodologías propuestas. En este apartado se citan algunas de estas acciones que se pretende ir desarrollando en los próximos años.

\subsubsection{Cambio de paradigma en la optimización energética de redes de riego}

Aunque las mejoras en los métodos de optimización energética de redes de riego han conseguido que estos puedan ser utilizados para la toma de decisiones en la operación diaria, todavía queda margen de mejora si se compara con las eficiencias computacionales alcanzadas mediante los métodos de programación lineal y los heurísticos. Por este motivo se contemplan dos acciones a realizar en el futuro.

Por un lado, se ha valorado efectuar la linealización del sistema de ecuaciones. A diferencia de los subsistemas de transporte, en este caso se han de evaluar en la función objetivo (o las restricciones) las pérdidas por fricción en cada línea para calcular la presión resultante en cada punto de consumo. La linealización en este caso podría ser causa de un error excesivo que haga inviable el método. Sin embargo, se estima, a priori, que redes con 
un dimensionamiento holgado puedan presentar un comportamiento que se asemeje lo suficiente al lineal y, en consecuencia, este método pueda ofrecer una buena solución con el beneficio de la gran eficiencia computacional de la programación lineal.

Por otro lado, también puede intentarse la definición de una buena heurística aprovechando el profundo conocimiento del problema a nivel ingenieril. La heurística podría observar indicadores como la sensibilidad de las presiones en los hidrantes a la activación de las tomas de modo que, en cada instante, se vayan añadiendo secuencialmente los riegos que mejor se adapten a la situación anterior, penalizando en menor medida a los puntos que más cerca estén de la presión crítica, y teniendo en cuenta la variación del rendimiento en la estación de bombeo. En principio, parece un reto difícil de abordar, pero es una línea que seguramente valga la pena explorar.

\subsubsection{Formulación del balance global de energías a lo largo de un periodo de tiempo}

En tratamiento de los depósitos en el método de cálculo de los flujos energéticos propuesto en el apartado 5.2 se basa en considerarlos análogos a las fuentes de suministro. Este tratamiento introduce una serie de complejidades y limitaciones que podrían evitarse si los depósitos se tratan como almacenes de agua y también de energía. El objeto del futuro trabajo es abordar el problema de los depósitos intermedios, y su papel real en el balance de energías, tanto a nivel instantáneo como a través del tiempo, lo que permitirá hacer un seguimiento detallado de la evolución de la energía en el sistema a nivel espacial y temporal, llegando a conclusiones y aplicaciones de gran interés, hasta ahora nunca exploradas.

La nueva formulación mantendría intacto el balance de energías a nivel de nudo, reduciendo las fuentes de suministro de agua únicamente a los embalses y a los puntos de inyección de caudal.

\subsubsection{Definición de nuevos indicadores energéticos}

El desglose efectuado sobre el origen y destino de cada una de las energías que intervienen en la red de suministro, incluso su efecto diferido a través de los depósitos intermedios permite definir una serie de nuevos indicadores energéticos de gran interés práctico para la toma de decisiones, los cuales no podrían ser evaluados sin aplicar la metodología propuesta en el apartado 5.2. Estos indicadores podrían llegar a detallar, por ejemplo, qué cantidad de energía de bombeo (o coste) se ha destinado a vencer desniveles o 
pérdidas, qué cantidad se ha invertido en la elevación útil de la presión de servicio, o incluso qué tuberías suponen un mayor coste operativo debido a sus pérdidas por fricción. Téngase en cuenta que ningún método de cálculo existente hoy en día es capaz de alcanzar este nivel de detalle.

\subsubsection{Mejora del sistema de filtrado de datos}

En primer lugar, derivado de la importancia que tiene la construcción de modelos hidráulicos que sean capaces de reproducir fielmente el comportamiento real del SDA, resultaría interesante el desarrollo de un método de filtrado más sofisticado que el presentado en el CAPÍTULO 6. Concretamente se ha pensado en tres mejoras que se podrían realizar.

Por un lado, el marcado de datos erróneos podría realizarse automáticamente, en lugar de tener que definir para cada variable los parámetros de detección definidos en el apartado 6.2. Se podría desarrollar una metodología capaz de clasificar automáticamente los datos observados en distintas categorías en base a las observaciones pasadas únicamente. Se propone hacer un análisis de los datos pasados basado en la agrupación (clustering) no supervisada, como los Modelos de Mezcla Gaussiana o el algoritmo k-Means, para determinar qué categorías contienen más muestras y, así, considerar las muestras que se distribuyen en dichas regiones como datos correctos, y la que queden alejadas de dichas regiones como datos anómalos.

Por otro lado, la generación de patrones podría sofisticarse sustituyendo el simple cálculo de medianas por la introducción de algún modelo de inteligencia artificial, como por ejemplo redes neuronales, que sean capaces de determinar la influencia que puedan ejercer factores externos, como la meteorología u otras variables del entorno, sobre cada una de las señales analizadas.

Finalmente, en el proceso de restitución de datos anómalos podría explotarse indirectamente el modelo hidráulico para establecer automáticamente las relaciones que existen entre las variables. En el procedimiento descrito en el apartado 6.3.3, se han de introducir manualmente los balances de caudal. Sin embargo, mediante el modelo hidráulico no solo podrían determinarse automáticamente las relaciones entre estos caudales, sino que se podrían generar relaciones adicionales incluyendo otro tipo de variables (presiones, niveles, etc.). 


\section{BIBLIOGRAFÍA}

Abadia, R., Rocamora, C., Ruiz, A., Puerto, H., 2008. Energy efficiency in irrigation distribution networks I: Theory. Biosyst. Eng. 101, 21-27. https://doi.org/10.1016/j.biosystemseng.2008.05.013

Alvarruiz, F., Martínez-Alzamora, F., Vidal, A.M., 2015. Improving the efficiency of the loop method for the simulation of water distribution systems. J. Water Resour. Plan. Manag. 141, 1-10. https://doi.org/10.1061/(ASCE)WR.1943-5452.0000539

Amdahl, G.M., 1967. Validity of the single processor approach to achieving large scale computing capabilities. Proc. April 18-20, 1967, spring Jt. Comput. Conf. - AFIPS '67 483. https://doi.org/10.1145/1465482.1465560

Arora, J.S., 2004. More on Numerical Methods for Constrained Optimum Design. Introd. to Optim. Des. 379-412. https://doi.org/10.1016/b978-012064155-0/50011-2

Barán, B., Von Lücken, C., Sotelo, A., 2005. Multi-objective pump scheduling optimisation using evolutionary strategies. Adv. Eng. Softw. 36, 39-47. https://doi.org/10.1016/j.advengsoft.2004.03.012

Bolognesi, A., Bragalli, C., Lenzi, C., Artina, S., 2014. Energy efficiency optimization in water distribution systems. Procedia Eng. 70, 181-190. https://doi.org/10.1016/j.proeng.2014.02.021

Bou Soler, V., 2016. Optimización en tiempo real del modo de operación de un abastecimiento de agua mediante técnicas metaheurísticas. Aplicación a la red de suministro a valencia y su área metropolitana. Universitat Politècnica de València.

Boulos, P.F., Altman, T., Jarrige, P.-A., Collevati, F., 1995. Discrete Simulation Approach for Network-Water-Quality Models. J. Water Resour. Plan. Manag. 121, 49-60.

Cabrera, E., Gomez, E., Cabrera Jr., E., Soriano, J., Espert, V., 2014. Energy Assessment of Pressurized Water Systems. J. Water Resour. Plan. Manag. 141, 1-12. https://doi.org/10.1061/(ASCE)WR.1943-5452.0000494.

Cabrera, E., Pardo, M., Cobacho, R., Cabrera Jr., E., 2010. Energy audit of water networks. J. Water Resour. Plan. Manag. 136, 669-677. https://doi.org/10.1061/(ASCE)WR.1943-5452.0000077 
CEDEX, 2017. Guía técnica sobre depósitos para abastecimiento de agua potable. Centro de Estudios y Experimentación de Obras Públicas. Madrid. ISBN: 978-84-7790-601-8

Deb, K., Pratap, A., Agarwal, S., Meyarivan, T., 2002. A fast and elitist multiobjective genetic algorithm: NSGA-II. IEEE Trans. Evol. Comput. 6, 182-197. https://doi.org/10.1109/4235.996017

Durillo, J.J., Nebro, A.J., 2011. JMetal: A Java framework for multi-objective optimization. Adv. Eng. Softw. 42, 760-771. https://doi.org/10.1016/j.advengsoft.2011.05.014

Dziedic, R., Karney, B.W., 2015. Energy metrics for water distribution system assessment: case study of the toronto network. J. Water Resour. Plan. Manag. 141, 491-501. https://doi.org/10.1061/(ASCE)WR.1943-5452

El-Omari, N.K.T., 2020. Sea Lion Optimization Algorithm for Solving the Maximum Flow Problem. IJCSNS Int. J. Comput. Sci. Netw. Secur. 20, 30. https://doi.org/10.22937/IJCSNS.2020.20.08.5

Eusuff, M.M., Lansey, K.E., 2003. Optimization of Water Distribution Network Design Using the Shuffled Frog Leaping Algorithm. J. Water Resour. Plan. Manag. 129, 210225. https://doi.org/10.1061/(ASCE)0733-9496(2003)129:3(210)

Fernández García, I., Rodríguez Díaz, J.A., Camacho Poyato, E., Montesinos, P., 2013. Optimal Operation of Pressurized Irrigation Networks with Several Supply Sources. Water Resour. Manag. 27, 2855-2869. https://doi.org/10.1007/s11269-013-0319-y

García, S., Faci, E., Aliod, R., Paño, J., Seral, P., García, A., 2015. Algoritmos y herramientas para la aplicación de estrategias de reducción de costes energéticos en sistemas de riego a presión, in: Actas de Las IV Jornadas de Ingeniería Del Agua. Córdoba, pp. 411-420.

Gay, L.F., Sinha, S.K., 2012. Measuring Energy Efficiency in Urban Water Systems Using a Mechanistic Approach 18, 139-145. https://doi.org/10.1061/(ASCE)IS.1943$555 X .0000072$.

Giacomello, C., Kapelan, Z., Nicolini, M., 2013. Fast Hybrid Optimisation Method for Effective Pump Scheduling. J. Water Resour. Plan. Manag. 193, 175-183. https://doi.org/10.1061/(ASCE)WR.1943-5452.0000239 
Grama, A., Gupta, A., Karypis, G., Kumar, V., 2003. Introduction to Parallel Computing; 2nd Edition. Search 856.

Gustafson, J.L., 1988. Reevaluating amdahl's law, in: Communications of the ACM. pp. $532-533$.

Hashemi, S., Filion, Y.R., Speight, V.L., 2015. Pipe-level energy metrics for energy assessment in water distribution networks. Procedia Eng. 119, 139-147. https://doi.org/10.1016/j.proeng.2015.08.864

Hutton, C.J., Kapelan, Z., Vamvakeridou-Lyroudia, L., Savić, D.A., 2014. Dealing with Uncertainty in Water Distribution System Models: A Framework for Real-Time Modeling and Data Assimilation. J. Water Resour. Plan. Manag. 140, 169-183. https://doi.org/10.1061/(asce)wr.1943-5452.0000325

Iglesias-Rey, P.L., Martínez-Solano, F.J., Ribelles-Aquilar, J. V., 2017. Extending EPANET Capabilities with Add-In Tools. Procedia Eng. 186, 626-634. https://doi.org/10.1016/j.proeng.2017.03.279

Jassadapakorn, C., Chongstitvatana, P., 2011. Self-Adaptation Mechanism to Control the Diversity of the Population in Genetic Algorithm. Int. J. Comput. Sci. Inf. Technol. 3, 90-96.

Jiménez-Bello, M.A., Martínez Alzamora, F., Bou Soler, V., Ayala, H.J.B., 2010. Methodology for grouping intakes of pressurised irrigation networks into sectors to minimise energy consumption. Biosyst. Eng. 105, 429-438. https://doi.org/10.1016/j.biosystemseng.2009.12.014

Jiménez-Bello, M.A., Royuela, A., Manzano, J., Martínez Alzamora, F., 2015. A methodology to improve water and energy use by network sectoring and proper irrigation scheduling, in: 7th IWA Intenational Conference on Efficient Use and Management of Water. Paris, pp. 1-11.

Jowitt, P.W., Germanopoulos, G., 1992. Optimal Pump Scheduling in Water Supply Networks. J. Water Resour. Plan. Manag. 118, 406-422. https://doi.org/10.1061/(ASCE)0733-9496(1992)118:4(406)

Langarita, R., Sánchez Chóliz, J., Sarasa, C., Duarte, R., Jiménez, S., 2017. Electricity costs 
in irrigated agriculture: A case study for an irrigation scheme in Spain. Renew. Sustain. Energy Rev. 68, 1008-1019. https://doi.org/10.1016/j.rser.2016.05.075

Lansey, K.E., Awumah, K., 1994. OPTIMAL PUMP OPERATIONS CONSIDERING PUMP SWITCHES. J. Water Resour. Plan. Manag. 120, 17-35.

Lin, M.H., Tsai, J.F., Yu, C.S., 2012. A review of deterministic optimization methods in engineering and management. Math. Probl. Eng. 2012. https://doi.org/10.1155/2012/756023

Liu, B., Cheng, C., Wang, S., Liao, S., Chau, K.W., Wu, X., Li, W., 2018. Parallel chanceconstrained dynamic programming for cascade hydropower system operation. Energy 165, 752-767. https://doi.org/10.1016/j.energy.2018.09.140

Mair, M., Rauch, W., Sitzenfrei, R., 2014. Improving incomplete water distribution system data. Procedia Eng. 70, 1055-1062. https://doi.org/10.1016/j.proeng.2014.02.117

Mamade, A., Loureiro, D., Covas, D., Alegre, H., 2014. Energy auditing as a tool for improving service efficiency of water supply systems. Procedia Eng. 89, 557-564. https://doi.org/10.1016/j.proeng.2014.11.478

Mamade, A., Sousa, C., Marques, A., Loureiro, D., Alegre, H., Covas, D., 2015. Energy auditing as a tool for outlining major inefficiencies: Results from a real water supply system. Procedia Eng. 119, 1098-1108. https://doi.org/10.1016/j.proeng.2015.08.9441

Moosavian, N., Lence, B.J., 2011. Nondominated Sorting Differential Evolution Algorithms for Multiobjective Optimization of Water Distribution Systems 1-9. https://doi.org/10.1061/(ASCE)WR.1943-5452.0000741.

Nia, M.B., Alipouri, Y., 2009. Speeding up the genetic algorithm convergence using sequential mutation and circular gene methods. ISDA 2009 - 9th Int. Conf. Intell. Syst. Des. Appl. 31-36. https://doi.org/10.1109/ISDA.2009.140

Ormsbee, L.E., Lansey, K.E., 1994. Optimal Control of Water Supply Pumping systems. J. Water Resour. Plan. 120, 237-252. https://doi.org/10.1016/0042-207X(85)90371-9

Pardo, M.A., Manzano, J., Cabrera, E., García-Serra, J., 2013. Energy audit of irrigation $\begin{array}{llll}\text { networks. } & \text { Biosyst. } & \text { Eng. }\end{array}$ https://doi.org/10.1016/j.biosystemseng.2013.02.005 
Pardo, M.A., Riquelme, A.J., Jodar-Abellan, A., Melgarejo, J., 2020. Water and energy demand management in pressurized irrigation networks. Water (Switzerland) 12. https://doi.org/10.3390/W12071878

Pelli, T., Hitz, H.U., 2000. Energy indicators and savings in water supply. J. / Am. Water Work. Assoc. 92, 55-62. https://doi.org/10.1111/1468-0076.00119

Pisinger, D., Ropke, S., 2007. A general heuristic for vehicle routing problems. Comput. Oper. Res. 34, 2403-2435. https://doi.org/10.1016/j.cor.2005.09.012

Pulido-Calvo, I., Gutiérrez-Estrada, J.C., 2011. Selection and operation of pumping stations of water distribution systems. Environ. Res. J. 5, 1-20.

Rasheed, K., Ni, X., Vattam, S., 2005. Methods for Using Surrogate Models to Speed Up Genetic Algorithm Optimization: Informed Operators and Genetic Engineering, in: Jin, Y. (Ed.), Knowledge Incorporation in Evolutionary Computation. Springer Berlin Heidelberg, Berlin, Heidelberg, pp. 103-122. https://doi.org/10.1007/978-3-54044511-1_6

Reca, J., Garcia-Manzano, A., Martínez, J., 2013. Optimal Pumping Scheduling for Complex Irrigation Water Distribution Systems. J. Water Resour. Plan. Manag. 140, 630-637. https://doi.org/10.1061/(ASCE)WR.1943-5452.0000360

Reca, J., García-manzano, A., Martínez, J., 2015. Optimal pumping scheduling model considering reservoir evaporation. Agric. Water Manag. 148, 250-257. https://doi.org/10.1016/j.agwat.2014.10.008

Rodríguez-Díaz, J.A., Pérez-Urrestarazu, L., Camacho-Poyato, E., Montesinos, P., 2011. The paradox of irrigation scheme modernization: more efficient water use linked to higher energy demand. Spanish J. Agric. Res. 9, 1000-1008. https://doi.org/10.5424/sjar/20110904-492-10

Rodríguez Díaz, J.A., López Luque, R., Carrillo Cobo, M.T., Montesinos, P., Camacho Poyato, E., 2009. Exploring energy saving scenarios for on-demand pressurised irrigation networks. Biosyst. Eng. 104, 552-561. https://doi.org/10.1016/j.biosystemseng.2009.09.001

Rodriguez, H., Puig, V., Flores, J.J., Lopez, R., 2017. Flow meter data validation and 
reconstruction using neural networks: Application to the Barcelona water network. 2016 Eur. Control Conf. ECC 2016 1746-1751. https://doi.org/10.1109/ECC.2016.7810543

Rossman, L.A., 2000. EPANET 2: users manual. Cincinnati US Environ. Prot. Agency Natl. Risk Manag. Res. Lab. 38, 200. https://doi.org/10.1177/0306312708089715

Rossman, L.A., Boulos, P.F., Altman, T., 1993. Discrete Volume-Element Method for Network Water-Quality Models. J. Water Resour. Plan. Manag. 119, 505-517.

Savic, D., Walters, G., 1997. Multiobjective genetic algorithms for pump scheduling in water supply. Evol. Comput. 1-9.

Seo, S., Gary M. Marsh, P.D., 2006. A review and comparison of methods for detecting outliersin univariate data sets. Dep. Biostat. Grad. Sch. Public Heal. 1-53.

Shi, H., You, F., 2016. Energy Optimization of Water Supply System Scheduling: Novel MINLP Model and Efficient Global Optimization Algorithm. AIChE J. 62, 4277-4296. https://doi.org/10.1002/aic

Sinha, R.S., Singh, Satvir, Singh, Sarabjeet, Banga, V.K., 2015. Speedup genetic algorithm using C-CUDA. Proc. - 2015 5th Int. Conf. Commun. Syst. Netw. Technol. CSNT 2015 1355-1359. https://doi.org/10.1109/CSNT.2015.148

Student, 1908. The probable error of a mean. Biometrika 6, 1-25. https://doi.org/10.1093/biomet/6.1.1

Todini, E., Pilati, S., 1988. Gradient Algorithm for the Analysis of Pipe Networks, in: Computer Applications in Water Supply. Volume 1: Systems Analysis and Simulation. p. 20.

Vegas Niño, O.T., Martínez Alzamora, F., Alonso Campos, J.C., Tzatchkov, V.G., 2017. Iniciación a la Programación con la Toolkit de Epanet v2.00.12 en un Entorno Windows, 1st ed. Instituto Mexicano de Tecnología del Agua, Jiutepec, México.

Wilkinson, R., 2000. Methodology for Analysis of the Energy Intensity of California's Water Systems and an Assessment of Multiple Potential Benefits Through Integrated Water- Energy Efficiency Measures. Calif. Inst. Energy Effic. 89. 